\title{
(In)Appropriate Online Behavior \\ A pragmatic analysis of message board relations
}

\author{
Jenny Arendholz
}

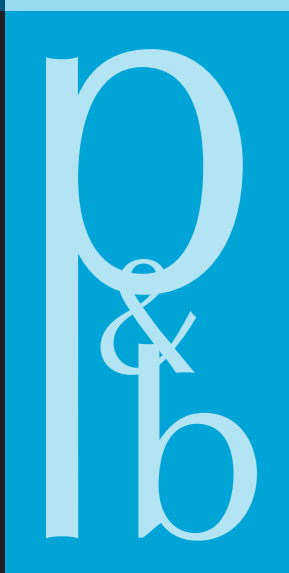

John Benjamins Publishing Company 
(In)Appropriate Online Behavior 


\section{Pragmatics \& Beyond New Series (PঊBNS)}

Pragmatics \& Beyond New Series is a continuation of Pragmatics \& Beyond and its Companion Series. The New Series offers a selection of high quality work covering the full richness of Pragmatics as an interdisciplinary field, within language sciences.

For an overview of all books published in this series, please see http://benjamins.com/catalog/pbns

\section{Editor}

Anita Fetzer

University of Augsburg

\section{Founding Editors}

Jacob L. Mey

University of Southern

Denmark

\section{Editorial Board}

Robyn Carston

University College London

Thorstein Fretheim

University of Trondheim

John C. Heritage

University of California at Los

Angeles

Susan C. Herring

Indiana University

Masako K. Hiraga

St. Paul's (Rikkyo) University

\section{Associate Editor}

Andreas H. Jucker

University of Zurich

\author{
Herman Parret \\ Belgian National Science \\ Foundation, Universities of \\ Louvain and Antwerp \\ Jef Verschueren \\ Belgian National Science \\ Foundation, \\ University of Antwerp
}

Sachiko Ide
Japan Women's University
Kuniyoshi Kataoka
Aichi University
Miriam A. Locher
Universität Basel
Sophia S.A. Marmaridou
University of Athens
Srikant Sarangi
Cardiff University
Marina Sbisà
University of Trieste

\section{Volume 229}

\author{
Deborah Schiffrin \\ Georgetown University \\ Paul Osamu Takahara \\ Kobe City University of \\ Foreign Studies \\ Sandra A. Thompson \\ University of California at \\ Santa Barbara \\ Teun A. van Dijk \\ Universitat Pompeu Fabra, \\ Barcelona \\ Yunxia Zhu \\ The University of Queensland
}

(In)Appropriate Online Behavior.

A pragmatic analysis of message board relations

by Jenny Arendholz 


\section{(In)Appropriate Online Behavior}

A pragmatic analysis of message board relations

Jenny Arendholz

University of Augsburg

John Benjamins Publishing Company

Amsterdam/Philadelphia 
The paper used in this publication meets the minimum requirements of the American National Standard for Information Sciences - Permanence of Paper for Printed Library Materials, ANSI z39.48-1984.

\section{Library of Congress Cataloging-in-Publication Data}

Arendholz, Jenny.

(In)Appropriate Online Behavior : a Pragmatic Analysis of Message Board Relations / Jenny Arendholz, University of Augsburg.

p. cm. (Pragmatics \& Beyond New Series, ISSN 0922-842X; v. 229)

Includes bibliographical references and index.

1. Electronic discussion groups--Social aspects. 2. Online etiquette--Social aspects. 3 . Discourse analysis--Data processing. 4. Discourse analysis--Technological innovations. 5. Online social networks--Social aspects. 6. Internet--Social aspects. 7. Language and the Internet. I. Title.

ZA4480.A73 2013

$302.30285--\mathrm{dc} 23$

2012041715

ISBN 9789027256348 (Hb; alk. paper)

ISBN 9789027272379 (Eb)

An electronic version of this book is freely available, thanks to the support of libraries working with Knowledge Unlatched. $\mathrm{KU}$ is a collaborative initiative designed to make high quality books Open Access for the public good.

The Open Access isbn for this book is 9789027272379.

(C) 2013 - John Benjamins B.V.

This e-book is licensed under a Creative Commons CC BY-NC-ND license. To view a copy of this license, visit https://creativecommons.org/licenses/by-nc-nd/4.o/. For any use beyond this license, please contact the publisher.

John Benjamins Publishing Co. · P.O. Box 36224 · 1020 ME Amsterdam · The Netherlands John Benjamins North America · P.O. Box 27519 • Philadelphia PA 19118-0519 • USA 
To my parents 



\section{Table of contents}

List of figures and tables $\quad$ XI

Acknowledgements $\quad$ XIII

CHAPTER 1

Introduction

CHAPTER 2

Communicating via networks I: A technical perspective

2.1 Introduction 5

2.2 A short history of hypertext and the Internet $\mathbf{6}$

2.3 Scientific disciplines and hypertext: CMC \& CMDA 10

2.4 In the spotlight: The ins and outs of message boards $\quad \mathbf{1 2}$

2.5 Reservations against the social potential of CMC 17

2.5.1 Technological determinism vs. social constructionism $\quad \mathbf{1 8}$

2.5.2 Cues-filtered-out approaches 19

2.6 Everything is not lost: The discovery of social potential within CMC

2.6.1 Opposed findings of more recent approaches $\quad 23$

2.6.2 Compensatory mechanisms and netlingo 25

2.7 Summary: Why CMC can be social after all 33

CHAPTER 3

Communicating via networks II: A social perspective

3.1 Introduction 37

3.2 Gathering online: In groups or communities? 38

3.3 Taking a look inside: The community of practice of The Student Room $\quad \mathbf{4 1}$

3.3.1 Social roles revisited: The hybridization of the private and public $\quad 42$

3.3.2 The emergence of norms and codes of conduct 46

3.4 Summary: Why we need to draw on FtF interaction 50 


\section{CHAPTER 4}

Interpersonal relations I: The origins of politeness, face \& facework

4.1 Introduction 53

4.2 Fraser, Lakoff and Leech: Some classic views on politeness 55

4.3 The face-saving view: Brown/Levinson's Politeness Theory 58

4.3.1 Key concepts and inner workings 59

4.3.2 The predecessor: Goffman's classic approach $\quad 66$

4.3.3 Brown/Levinson vs. Goffman: Some interrelations 71

4.4 Summary: What to keep and what to drop 75

\section{CHAPTER 5}

Interpersonal relations II: Putting (im)politeness

in an integrative perspective

5.1 Introduction 79

5.2 A working definition of politeness 80

5.3 Locher/Watts' comprehensive framework of interpersonal relations $\quad \mathbf{8 3}$

5.4 Impoliteness: The neglected stepbrother of politeness $\quad 89$

5.5 Distinguishing types of inappropriate behavior:

Impoliteness vs. rudeness 95

5.6 Appropriate impoliteness: Mock-impoliteness and banter 97

5.7 Strings of inappropriate online behavior: Flaming 100

5.8 Summary: Implications of an integrative perspective for the analysis 102

CHAPTER 6

Prelude to the analysis: Gathering contextual factors

6.1 Introduction 105

6.2 The participant: Personal contextual factors 107

6.2.1 Face constitution in online message boards 113

6.2.2 Trolling: A means of strategic deception 121

6.3 The relations: Interpersonal contextual factors $\mathbf{1 2 2}$

6.4 The setting: (Extra-) medial environmental factors $\mathbf{1 2 5}$

6.5 Summary: A comprehensive framework of context in message boards 


\section{CHAPTER 7}

Analyzing online message boards I: Thread starts

7.1 Introduction $\mathbf{1 2 7}$

7.2 Aspects of corpus compilation and its internal structure $\mathbf{1 3 0}$

7.3 Establishing interpersonal relations online 137

7.3.1 What's in a heading? 138

7.3.2 The initiators: Investigating thread starters' face claims $\mathbf{1 4 0}$

7.3.3 Face claims in first posts $\mathbf{1 4 5}$

7.3.4 Laying the groundwork for thread discussions: First entries $\quad \mathbf{1 4 8}$

7.4 Other hotbeds for establishing contact: Profiles and signatures $\mathbf{1 5 7}$

7.5 Summary: Findings for the establishment of interpersonal relations

CHAPTER 8

Analyzing online message boards II: Thread interaction

8.1 Introduction $\mathbf{1 6 3}$

8.2 Managing interpersonal relations online $\quad 167$

8.2.1 Getting to know others 168

8.2.2 Looking for like-minded others $\mathbf{1 8 2}$

8.2.3 Asking for advice 197

8.2.4 Asking for opinions 206

8.2.5 Blowing off steam $\mathbf{2 2 6}$

8.2.6 Offering advice/information $\quad 238$

8.3 Summary: Findings for the management of interpersonal relations

CHAPTER 9

Conclusion

Bibliography

Name index

Subject index 



\section{List of figures and tables}

Figure 1. Structure of a typical forum homepage (URL 4) $\quad \mathbf{1 4}$

Figure 2. Structure of the homepage of the message board The Student Room (URL 5) 15

Figure 3. List of threads within a subtopic in The Student Room (URL 6) 15

Figure 4. Beginning of a thread in the message board The Student Room (URL 7) 16

Figure 5. Standard TSR header of posts to identify a user (left side) 29

Figure 6. User's homepage on twitter, redirected from the drop-down menu 30

Figure 7. Standard TSR header of posts to identify a user (right side) $3 \mathbf{1}$

Figure 8. Selection of signatures in TSR 32

Figure 9. Prototypical TSR post with integral and accompanying components 33

Figure 10. Four directions of FTAs according to Brown/Levinson $\quad \mathbf{6 1}$

Figure 11. Schematic representation of Goffman's notion of face $\quad 72$

Figure 12. Schematic representation of Brown/Levinson's notion of face 73

Figure 13. Schematic representation of a combined model of face $\quad 76$

Figure 14. Cognitive processes of individual value judgments about relational work $\quad \mathbf{8 8}$

Figure 15. Cycle of face negotiation $\quad \mathbf{1 1 7}$

Figure 16. Percentages of contributions per user (C/User) $\quad \mathbf{1 3 6}$

Figure 17. User types according to frequency of contribution (in percentages) $\quad \mathbf{1 3 6}$

Figure 18. Two strands of investigation: facework and relational work $\quad 163$

Figure 19. Discourse structure and types of interactivity in two prototypical threads $\quad \mathbf{1 6 4}$

Figure 20. Threads according to their interaction quotient (IQ) in percentages $\quad 166$

Figure 21. Percentages of relational work in the entire message board corpus 253

Table 1. Types of message board recipients (Goffman 1981, modified) 44

Table 2. Lines of investigating politeness $\quad 55$

Table 3. Lakoff's two-sided model of pragmatic competence (Watts 2003:60, adapted) 57

Table 4. Brown/Levinson's five superstrategies for the mitigation of FTAs (1987:60, 69) $\mathbf{6 2}$

Table 5. Framework of relational work (Locher/Watts 2005: 12, adapted) $\quad \mathbf{8 5}$

Table 6. Lachenicht's strategies to modify FTAs (1980:621, my emphasis) 91

Table 7. Types of impoliteness according to face-orientation 96

Table 8. Modified model of relational work $\quad 97$

Table 9. Three dimensions of context $\quad 107$

Table 10. Message board structures for self-portrayal $\quad \mathbf{1 1 1}$

Table 11. Users' options for self-portrayal online $\quad 112$

Table 12. Contextual and environmental variables in message boards $\quad \mathbf{1 2 6}$

Table 13. Internal structure of the message board corpus $\quad 132$

Table 14. Sum total of contributions per user $(C)$ in relation to their occurrence $(O) \quad \mathbf{1 3 5}$

Table 15. User types according to frequency of contribution $\quad \mathbf{1 3 6}$

Table 16. Thread titles (headings) according to their communicative purpose $\quad 137$ 
Table 17. Thread starters as "do-gooders" and "trouble makers" $\quad \mathbf{1 4 4}$

Table 18. Grouping of the threads according to their interaction quotient (IQ) $\quad 165$

$\begin{array}{ll}\text { Table 19. Relational work in thread \#1 } & \mathbf{1 7 4}\end{array}$

Table 20. Relational work in thread \#2 $\quad \mathbf{1 8 1}$

Table 21. Relational work in the first category (Getting to know others) $\mathbf{1 8 2}$

Table 22. Relational work in thread \#19 190

Table 23. Relational work in thread \#18 195

Table 24. Relational work in the second category (Looking for like-minded others) 197

Table 25. Relational work in thread \#14 202

Table 26. Relational work in thread \#11 203

Table 27. Relational work in the third category (Asking for advice) 205

Table 28. Relational work in thread \#13 $\mathbf{2 1 3}$

Table 29. Relational work in the forth category (Asking for opinions) 225

Table 30. Relational work in thread \#12 231

Table 31. Relational work in thread \#17 232

Table 32. Relational work in thread \#26 237

Table 33. Relational work in the fifth category (Blowing off steam) $\quad 237$

Table 34. Relational work in thread \#48 $\mathbf{2 4 2}$

Table 35. Relational work in the sixth category (Offering advice/information) 247

Table 36. Availability versus trustworthiness of evaluations in type A and $B_{t}$ posts $\quad 251$

Table 37. Relational work in all 50 threads $\quad 252$

Table 38. Incidences of relational work in the entire message board corpus 253

Table 39. Incidences of flaming in all six categories $\quad \mathbf{2 5 4}$

Table 40. Types of relational work used in flames $\quad 254$

Table 41. Percentages of relational work according to categories $\quad \mathbf{2 5 6}$

Table 42. Ranking of the six categories according to the appropriateness of behavior $\quad 257$ 


\section{Acknowledgements}

This book could not have been written without contributions of various kinds from many people. First and foremost, I would like to thank my supervisor Wolfram Bublitz for supporting me and my work from the beginning to the end. It was his personal and professional guidance that made me believe in the success of this thesis. I am no less indebted to my second supervisor Dieter Götz for contributing fruitful perspectives and helpful comments. I am very thankful for his kind and flexible assistance in this project.

I am also deeply grateful to my dear colleagues and friends Christian Hoffmann, Volker Eisenlauer and Monika Kirner, who took the time to discuss and read through numerous drafts of chapters and who gave me valuable help in shaping and arranging my thoughts. On a related note, I would also like to express my thanks to all the other members of the doctoral colloquium Olena Vorontsova, Iris Zimmermann, Elisabeth Fritz - and again to Wolfram Bublitz for his hospitality - as well as Anne Troeltsch and Siaohui Kok, all of whom detected typos, grammatical flaws and logical inconsistencies diligently.

Further, my thanks go to the kind souls in the secretary's offices, especially to Monika Martens, Gudrun Nelle and Inge Christopher, who generously and constantly provided moral support, encouragement and the most delicious chocolate. I would also like to thank our student assistants and Johannes Bleimeir in particular, who has been a great help in compiling the message board corpus and in drawing up descriptive charts.

Most importantly, though, I would like to express my gratitude to my whole family, my parents Karin and Jürgen Arendholz and my grandparents Vera and Richard Arendholz, for their unwavering and unconditional moral and financial support. Finally, I would also like to thank my husband Frank, who has been a constant source of inspiration and calmness and who secured my mental and physical wellbeing with heartfelt encouragement and good food.

Needless to say, none of the above is in any way to blame for any remaining flaws or inadequacies, as I remain responsible for them.

Jenny Arendholz

Augsburg, August 2012 



\section{CHAPTER 1}

\section{Introduction}

The opinions which we hold of one another, our relations with friends and kinsfolk are in no sense permanent, save in appearance, but are as eternally fluid as the sea itself.

(Marcel Proust)

As is usually the case with comparatively young disciplines, at the outset there are a lot of myths. In the case of the early days of computer-mediated communication (CMC) about two decades ago, the Internet was suspected to be ill-suited for the transmission of social content and, even worse, to promote uninhibited and rude behavior. In fact, these clichés are widespread and extremely tenacious to this very day and are still propagated by various press releases, which every now and then call for and suggest an etiquette for different types of communication via computers (see URL 1,2). This claim should, however, be handled with extreme care for various reasons: For one thing, it contains a careless and invalid generalization when talking about "the" Internet. We are not, however, dealing with one homogeneous type of mediated communication but with a number of diverse and individual subtypes thereof. Instead of investigating "the" Internet, we are in fact looking into chats, weblogs, email conversations and - in the case of this study - online message boards like The Student Room (TSR), a discussion platform for British and international students. For another thing, uninhibited and rude behavior as well as their more desirable counterpart, polite behavior, are not homogeneous phenomena either but rather fuzzy concepts, which are difficult to grasp.

One thing we can be sure of, however, is the nature of communication as such: No matter if we interact with each other face-to-face or mediated via computers, our utterances always encompass two separate but nevertheless interrelated sides: We convey information on an ideational level, while we (un)consciously negotiate social relations on an interpersonal plane at the same time (cf. Watzlawick et al. 1967; Halliday 1978) - a pragmatic dichotomy which has also been labeled transactional vs. interactional function of language by Brown/Yule (1983) and message vs. metamessage by Tannen (1992). Accordingly, we are not only busy doing Inhaltsarbeit but also Beziehungsarbeit (relational work), a distinction proposed by Bublitz (2009), with the latter subsuming the process of manipulating interpersonal relations in whatever way or direction. Admittedly, either of the two 
sides of communication may be foregrounded according to the purpose of interaction, the ideational level in university lectures, to give but one example, or the interpersonal level in small talk - with the respective other still being present. In any event and independent of the channel used for transmission, there is always an interpersonal aspect integrated in our utterances. Comments like "It's not what you said but the way that you said it" can be explained with recourse to this distinction, since how or even that we say what we say always communicates social, interpersonal meaning as well (Tannen 1992: 14).

These preliminary and as of yet broad-brush considerations, which will be revisited in later chapters, introduce the two basic components of the object of this study: online message boards and the interpersonal relations to be found therein. The combination of these two leads to the general objective of this study, which can be phrased as follows: How are interpersonal relations established, managed and negotiated in online message boards?

The very nature of interpersonal relations itself poses, however, a serious stumbling block for this kind of survey: As expressed in the above quote by Proust, we are dealing with an extremely unstable and ephemeral phenomenon, which is based on subjective and spontaneous value judgments and which is thus always in a state of flux. Consequently, the quality of interpersonal relations lies more often than not in the oft-cited eye of the beholder. What is more, interpersonal relations cannot simply be read off textual surface structures, but are subject to constant negotiations within actual, socially constructed discourse. This holds especially true for asynchronous message boards, where social institutions such as communities are mainly shaped through discourse alone, as users do neither share geographical space nor time (Rheingold 1993; Kollock/Smith 1999). On top of that, and true to Humboldt's (1820) maxim "Es giebt nichts Einzelnes in der Sprache", the process of creating and managing interpersonal relations is strongly influenced by an almost indeterminable number of contextual factors.

It is thus imperative to first pin down the ins and outs of message boards and even more so the fuzzy field of interpersonal relations, including its key components and influencing factors, before delving into empirical analyses. Both of these two basic strands of investigation shall help to operationalize the rather broad objective appropriately. They are mirrored in the following research questions, which will also serve as a guideline for the course of this study:

A. The descriptive part

1. What are the technological and social prerequisites and mechanisms of communication in online message boards like The Student Room (TSR)? How are they made use of? 
2. How can key (as well as marginal) concepts of interpersonal relations be defined and clustered adequately, especially in view of analyzing online communication in message boards?

B. The empirical part

3. Which contextual factors need to be included when evaluating users' online behavior? Where can we find them in the message board system of The Student Room (TSR)?

4. How are interpersonal relations formally expressed and interactively negotiated by experienced and new message board members? How many incidences of (the various types of) positively and negatively marked behavior can be found?

The first research question within the descriptive part will be answered in Chapter 2 and 3, while the second one will be dealt with in detail in Chapters 4 and 5 . For the purpose of illuminating the whole panoply of interpersonal relations, condensed state of the art reports of key concepts such as politeness, impoliteness, rudeness and flaming as well as outlines of rather marginal concepts like mock-politeness, mock-impoliteness and banter will be introduced, before being assigned a place within the larger framework of relational work. Chapter 6 marks the transition to the empirical part of this study: In it, contextual factors, which can become influential for users' as well as analysts' evaluations of interpersonal relations, are compiled and partly exemplified. This is done with the help of excerpts taken from a message board corpus, which is based on The Student Room (TSR) and which was specifically designed for the present study. The keystone of this study is represented by Chapters 7 and 8 , which both expose and illustrate mechanisms for the establishment and the negotiation of interpersonal relations. To this end, the empirical analysis will not only compile (medium-specific) strategies but also sound out the general atmosphere and participants' interpersonal conduct in said corpus.

As the nature of this subject only allows for a very limited amount of quantitative research, the corpus analysis will be predominantly qualitative, exhibiting a pragmatic and partly sociolinguistic orientation. This methodology is best described by Herring (2004:339):

The specific approach to computer-mediated discourse analysis [...] is informed by a linguistic perspective. That is, it views online behavior through the lens of language, and its interpretations are grounded in observations about language and language use.

Finally, Chapter 9 will summarize and evaluate the results of the empirical part with recourse to the theoretical considerations of previous chapters. 
The raison dêtre of this study is manifold: Recent developments in online message boards shall be accounted for in order to gain specific insights into this as yet mostly unattended type of computer-mediated communication. Above that, a structured, comprehensive and, above all, critical overview of the complex field of interpersonal relations shall be given. Most importantly, though, an up-to-date, realistic and empirically valid picture of interpersonal processes and behavior in online communities endemic in message boards shall be drawn, in order to do away with unjustified and obsolete myths that have been wandering through scientific literature far too long. 


\title{
Communicating via networks I
}

\author{
A technical perspective
}

\subsection{Introduction}

Wholly new forms of encyclopedias will appear, ready made with a mesh of associative trails running through them [...].

(Bush 1945)

As early as 1945 and almost half a century before its actual launching, Vannevar Bush gave an astonishingly accurate description of what is commonly called "the Internet" these days. This latest technological masterstroke is still gaining momentum in today's information society as it has constantly extended its span of functions and applicability over the past decades and has evolved from a simple tool on military and academic desks to one of the most important communication facilities in the middle of our homes. But how do we, in fact, communicate via computers? Do we chitchat online in the same way as we do "live", meaning faceto-face (henceforth FtF)? Before we can even think of investigating this mediated form of communication and the inner workings of interpersonal relations created and maintained through it, we need to unfold the basic prerequisites of "going online" in the first place. Despite its enormous popularity, only few users do in fact know that it is not the Internet per se which allows them to communicate with people at all times and all over the world, but the World Wide Web (WWW).

Although the distinction may be minor for users, researchers do not skid over such details, which is why this chapter puts emphasis on purely technical considerations to begin with. It also serves as a springboard for the social perspective adopted in the following chapter. The aim of Section 2.2 is thus to shed light on the historic development of the Internet, the WWW and its essential communication vehicle, hypertext. Subsequently, networked communication will be displayed as the cornerstone of disciplines such as computer-mediated communication (henceforth $\mathrm{CMC}$ ) and computer-mediated discourse analysis (henceforth CMDA). Section 2.4 details the structure of one type of CMC, online message boards, concentrating especially on The Student Room (TSR), the message board system which will serve as the basis for the empirical section of this book. The ensuing chapter deals with an extremely pressing issue, as it gives an in-depth description of doubts laid on the social potential of networked communication - a 
skepticism which finds expression in two separate views, technological determinism and the so-called cues-filtered-out approaches. These two pessimistic views will be run counter in Section 2.6, which puts the social potential of CMC into perspective by outlining those mistakes of former approaches that led to the false assumptions about CMC in the first place and that need to be avoided in the analysis to come. Finally, the summary addresses the fact that CMC can be social after all, thus setting the stage for the third chapter.

\subsection{A short history of hypertext and the Internet}

[H]ypertexts reflect the nature of the human mind itself [and] because we think associatively, not linearly, hypertext allows us to write as we think. (Bolter 2001:42)

One of the most important, if not the most important foundation stones of the Internet was the groundbreaking idea of hypertext, which paved the way for the creation and development of CMC in the first place. Originally born out of the desire to technically support the human brain at information processing and problem solving, the idea of hypertext was first brought up by the American engineer Vannevar Bush during the 1930s and finally published in his 1945 paper "As we may think". In it, Bush describes the Memex (Memory Expander), a "device in which an individual stores all his books, records, and communications, and which is mechanized so that it may be consulted with exceeding speed and flexibility. It is an enlarged intimate supplement to his memory" (1945: n.p.). Accordingly, the Memex was supposed to resemble the human brain as an "intricate web of trails" (ibid.), capable of tying items together in a way that one item can lead to any other item immediately. Although this system was not based on computer-technology yet, it is nevertheless often quoted as the ideational grandfather of today's hypertext technology, since it already contained its key concept: linking documents thematically as well as functionally via technical processes (Storrer 2008:212f.).

It was the American sociologist and philosopher Ted Nelson, who, in his talk "As we will think", picked up Bush's idea of the Memex in 1972, especially focusing on the concept of trails in order to utilize it for text production processes. It was also Nelson, who, in 1963, gave this new technique of producing and receiving texts its name, hypertext. It was around that time that Nelson founded project Xanadu, the first and still ongoing hypertext project, which was dedicated to the establishment of a user friendly hypertext system. ${ }^{1}$ Indeed very similar to today's

1. Nelson's enduring efforts are documented in his monographs Computer Lib/Dream Machines (1974) and Literary Machines (1981). 
WWW, it was not only supposed to be stored decentrally but it was also meant to be available worldwide. However, project Xanadu has not come to fruition yet.

The first one to instantiate a truly operative hypertext system - also inspired by Bush - was yet another American computer pioneer, Douglas C. Engelbart, best known for the invention of the computer mouse as an input device. His new system, AUGMENT, was the result of a research agenda which was titled "Augmenting Human Intellect: A Conceptual Framework". True to its name, AUGMENT was not meant to replace or automate human thought processes but to augment them. Since Engelbart's Augmentation Research Center (ARC) was funded by the ARPA (Advanced Research Projects Agency), his new online system soon became involved with the well-known ARPANET. It was established in 1969 and interconnected two research centers: a lab at UCLA and Engelbart's lab at Stanford, which were later joined by two other American research facilities (UCSB and the University of Utah).

The early backbone of the first Internet was thus created, further facilitating the transfer of computer programs and data between remote computers of geographically dispersed groups of scientists - years later, not only for academic and business related purposes, but also in the interest of national defense (cf. Rheingold 1993). The early 1970s eventually saw the development of computer networks as a means of communication among computer scientists (see also 2.4). The last step to popular use finally took place in the 1990s. Today, the Internet is an almost global network which connects millions of computers by using a number of agreed formats, so-called protocols. In this process, the most crucial role is played by the World Wide Web (WWW), developed in 1989 and made publicly accessible in 1993 by the computer scientists Tim Berners-Lee and Robert Cailliau at the European Organization for Nuclear Research CERN in Geneva. It is important to note that, although often used synonymously, the WWW is only a part of the Internet - as Thurlow et al. (2004:29) remarked, "the Web is a technology within a technology" or put figuratively, "the Web is to the Internet what language is to communication: it cannot account for everything we do, but it is unquestionably a major element in the larger system". The WWW is a system of computer servers which are connected through the Internet to support the exchange of files which are formatted mostly in HTML (hypertext markup language).

The basic unit of the WWW to this very day is hypertext, which has been the subject of scientific interest - and accordingly various definitional approaches ever since its inauguration. In 1992, Landow gave this technological definition of hypertext, which is

text composed of blocks of words (or images) linked electronically by multiple paths, chains or trails in an open-ended, perpetually unfinished textuality described by the terms link, node, network, web and path.

(1992:3) 
Another rather vivid description of hypertext is proposed by Bolter (2001:35):

A hypertext is like a printed book that the author has attacked with a pair of scissors and cut into convenient verbal sizes [- only] that the electronic hypertext does not simply dissolve into a disordered heap, because the author also defines a scheme of electronic connections to indicate relationships among the slips.

With regard to the comparison drawn between hypertexts and traditional (printed) texts, especially (text) linguists were eager to find out the true nature of this new kind of text. ${ }^{2}$ Consequently, they wanted to solve the arising question in what way hypertexts can be distinguished from conventional (printed) texts. The remainder of this chapter therefore briefly pinpoints the four most important claims which are often put forward to illustrate key characteristics of hypertexts and to delimit them from comparatively "old-fashioned" printed texts: ${ }^{3}$

\section{Claim 1: Hypertexts can be multi-linear}

Following Storrer's basic definition, hypertexts are "nicht-lineare Texte" (2008:215ff.) (corresponding to non-sequential texts), which need to be administrated with the help of computer-technology. For the sake of illustration, Nelson remarks that "hypertext [...] is text structure that cannot be conveniently printed" (1972:253). In order to get yet a clearer picture, we need to distinguish between the production and the reception side of communication, which is why the notions of compositional linearity and perceptive linearity (Hoffmann 2012:47) appear to be useful. With regard to these two notions, conventional texts are relatively static in that authors suggest an optimal reading path (compositional linearity) which is normally followed by the audience (perceptive linearity): Usually, we read a novel from cover to cover and do not decide to read the second chapter before the first, although there exists at least the theoretical possibility to do so. ${ }^{4}$ Hypertexts, by their very nature, are characterized by a highly fragmentary organization as they are more flexible and do not necessarily exhibit a compositional linearity: Creators of webpages do not usually tell us which link to click first - the exception, of course, being some kinds of guided tours through networked pages. As there

2. For a detailed and critical discussion surrounding the question whether de Beaugrande/ Dressler's (1981) criteria for the differentiation of texts and non-texts can also be applied for a classification of hypertexts, see, for example Vater (1992:19) or Sandig (2000:93).

3. Further discussions about the characteristics of hypertext can also be found in Eisenlauer/ Hoffmann (2008), Bublitz (2005, 2008) and Huber (2000).

4. Exceptions to this rule can be found in game books (such as Sugarcane Island) and dictionaries or encyclopedias, in which the reader is encouraged or even supposed to choose his own individual reading path. 
are many links to choose from, users, on the other hand, are confronted with a multitude of possible reading paths, depending on their interests and likings. For this reason, most hypertexts are characterized as multi-linear.

\section{Claim 2: Hypertexts can be coded multimodally}

Unlike printed texts, hypertexts can be composed of more than just written texts, as other representational formats $\left(\right.$ or modes ${ }^{5}$ ), such as pictures, audio or video files, can also be integrated in hypertext documents or be interconnected via hyperlinks. Since traditional texts such as newspapers, to give but one example, also employ formats other than language (e.g. pictures), we have to keep in mind that multimodality is not necessarily a unique feature of hypertexts - although the technological possibilities of computer-mediated texts perfectly lend themselves to multimodal use. This provides both producing and receiving users with a new communicative potential. According to Nelson, hypertexts, which use more than one channel for the communication of information, can therefore be referred to as hypermedia. ${ }^{6}$ Other labels have been assigned to hypertext, for example by Bolter (1991), who speaks of "synaesthetic texts". As Bublitz remarks, hypertext "refers to a much wider concept than text; indeed, it incorporates text as one of its components" (2008:259).

\section{Claim 3: Hypertexts can be dynamic}

The content of hypertext is rarely static, but subject to constant change and extension to meet the ever emerging user needs. Consequently, it is not unlikely that

5. In this study, I follow Holly (1997) and Hoffmann (2012) in using the notion of medium for tangible, technological means or carrier devices, such as the computer or books, which create, intensify, store and transport (linguistic) signs over great distances (Holly 1997: 69f.). The term form of communication, however, is reserved for virtual and multifunctional categories such as message boards and other software services which are delimited through text-external configurations like the number of participants, the temporal dimension (synchronous/asynchronous) or the direction of communication (monologic/dialogic). Finally, modes or channels account for the distinction between spoken and written discourse, including all kinds of representational formats such as pictures, audio or video files. Note, however, that the majority of the approaches quoted in this chapter do not sustain this differentiation, but use the term medium instead. For comprehensive discussions dealing with these different semiotic systems see also Herring (1999, 2001, 2007), McLuhan (1964), Murray (1988) and Stöckl (2004, 2006).

6. The notions hypertext and hypermedia are often used synonymously (cf. Kuhlen 1991). Note, however, that Storrer (2008:220) distinguishes carefully between hypertexts and e-texts. She reserves the latter notion for those types of texts, which - although being available online - exhibit typical characteristics of printed texts (e.g. sequential organization) and can be understood as replicas of conventional (printed) publications (e.g. scientific articles). For this reason, e-texts cannot be considered hypertexts in the sense of Storrer's basic definition. 
webpages vanish into thin air over night never to be seen again. This feature must, of course, also be taken into consideration when investigating hypertexts.

\section{Claim 4: Hypertexts can be interactive}

The term interactivity is used to describe a feature of the software which enables it to react to users' demands in predetermined ways and thus alludes to the cooperation between machines and humans. Above that, and even more importantly, interactivity is not only a feature of hypertexts but of all kinds of computational applications which allow users to manipulate their course of action, such as computer games and learning programs. Interactivity can include a range of simple operations such as clicking on links or entering search keywords (cf. Storrer 2008:219).

\subsection{Scientific disciplines and hypertext: CMC \& CMDA}

As we have seen before, web browsers such as Internet Explorer or Firefox can be used for much more than just data transfer and information retrieval. As a matter of fact, the communicative component has become more and more important in recent years, thereby earning the WWW a new sphere of scientific research: computer-mediated communication (CMC). Lying at the intersection of several disciplines, among them computer science, systems science, organizational theory, social psychology as well as sociolinguistics and (linguistic) pragmatics, the field of CMC is dedicated to the research of the numerous aspects of online communication. A rather broad definition is thus proposed by December (1997), who - in agreeing with Herring (1996) - claims that CMC "is a process of human communication via computers, involving people, situated in particular contexts, engaging in processes to shape media for a variety of purposes". A social scientific definition, which appears to be more relevant considering the focus of investigation of this study, is presented by Stephen (1991:236), who considers $\mathrm{CMC}$ research to be

the study in which the ways in which the computer as a communication medium may influence social psychological/processes [sic!] occurring between human communicators. Today's CMC research analyzes new social practices (for example, computer conferencing) enabled by institutional, national or global computer communication networks and the consequences of various characteristics of communication that occur uniquely or more frequently in this context (e.g. anonymous exchange, asynchronous interaction, exclusive reliance on written messages, etc.). 
These postulated new "social practices", which are supposed to be observable when users exchange ideas with other users, can be witnessed in the most diverse forms of communication within CMC, which encompass synchronous communication platforms such as web chats, instant messaging and MUDs ${ }^{7}$ as well as asynchronous email, fora, message boards, and weblogs. The common differentiation between synchronous and asynchronous types of CMC refers to the fact that some communication platforms require users to be simultaneously present when interacting with each other online. Asynchronous exchanges, in contrast, are not at all tied to time and can be taken up, interrupted or ended whenever the user feels like it or is able to fit it into his schedule without disrupting the conversational flow. This does not, however, mean that certain asynchronous types of CMC cannot be utilized for synchronous purposes as well: One might easily imagine the case in which a message board or a forum is used synchronously by two or more interlocutors who happen to be online at the same time to discuss a place to meet for the night.

Computer-mediated communication is predominantly text-based in that messages are typed on computer keyboards and read as texts on computer screens - normally, but not always, at two different locations. Consequently, the production and the reception of a message, which usually happen at once in FtF contexts, are automatically split up into two separate and temporally distinct acts. For this reason, Beißwenger $(2002,2003)$ and others even treat chats as cases of quasi-synchronous communication. Although activities dominating new electronic forms of communication very much resemble familiar technologies, e.g. the telephone or the typewriter, their convergence in the digital sphere provides users with entirely new possibilities (cf. Culnan/Markus 1987:422).

As a matter of fact, it is this text-based communication that presents itself as a fascinating new subject of interest not only for linguistic pragmatics. Accordingly labeled as computer-mediated discourse (CMD), it is defined by Herring as "the communication produced when human beings interact with one another by transmitting messages via networked computers" (2001:612). In comparison to the broader interdisciplinary study of CMC, the analysis of CMD, termed computer-mediated discourse analysis (CMDA), focuses specifically "on language and language use in computer networked environments [and makes] use of methods of discourse analysis (ibid., original emphasis). CMDA comprises "any analysis of online behavior that is grounded in empirical, textual observations" (Herring 2004:339) and is based on two major influencing factors: the technological

7. The acronym MUD is short for multi-user dungeon and stands for a multi-user virtual world similar to role-playing games, in which players get together in real-time for fun and games, as well as for communicating with each other. 
prerequisites of the medium (and of the form of communication) as well as the social constraints of the situation (Herring 2007: 10). Although the field obtained its name only in 1995 (cf. Herring 2001:613), CMD analyses have already been conducted since the mid-1980s - a claim which is supported by linguistic works of Murray (1988) and Severinson Eklundh (1986), to quote but two (cf. Herring 2004: 340). In structuring the scope of CMDA, Herring illustrates that CMDA has already been used for the investigation of numerous linguistic phenomena on a micro-level (e.g. online word-formation, lexical choice, sentence structure, and code switching) as well as on a macro-level (e.g. coherence, community, gender equity, and identity).

\subsection{In the spotlight: The ins and outs of message boards}

So far, we have established that CMC serves as an umbrella term for an extremely diversified range of forms of communication in terms of technological makeup as well as potential communicative functions and corresponding social dealings. To measure every one of them with the same yardstick would be inadequate, resulting in unqualified and invalid overgeneralizations. This section will therefore narrow the focus to message boards as the object of investigation, in order to establish some basic technological facts and inner workings for the empirical part of this work.

Nowadays, sections for discussion are an integral part of almost every website. Not only do the most important web portals like Yahoo@, Google $\odot$ and AOL( maintain extensive (moderated or unmoderated ${ }^{8}$ ) fora and/or message boards, these web applications are also provided by institutions like universities and libraries, companies, TV channels, online data bases and so on. Although they may have a common goal in helping users connect with each other, they differ not only in layout and structure. More strikingly, the multitude of topics and fields of interest discussed in these web applications almost seems to correspond with the large repertoire of their aliases: Going online, you are not only invited to join Internet, Web or discussion forums/fora, ${ }^{9}$ but also message or discussion boards, bulletin boards, (electronic) discussion groups, and newsgroups.

Unfortunately, we cannot fall back on scientific literature to help us circumscribe these different notions, as we are yet dealing with a largely uninvestigated

8. In a moderated discussion platform such as TSR (see next chapter), a supervising authority, a moderator, has to approve of the appropriateness of entries before they are indeed posted.

9. Note that even the formation of the plural poses a problem. This work will give preference to the Latin plural. 
subject. Except for practical (online) guides, advice and support services on how to access, join or create one of the aforementioned electronic discussion platforms (cf. Bins/Piwinger 1997; Donnerhacke 1996; Fabrot 2001; Jasper 1997), the amount of scientific let alone linguistic literature concerning basic notions and structures of fora and message boards is rather thin (cf. Münz 2002). A great part of literature focuses on the technical side of online communication, mostly stressing computer programming. On the other hand, general introductions into CMC (cf. Androutsopoulos et al. 2006; Beißwenger et al. 2004; Burger 2005; Crystal 2001; Herring 1996; Kuhlen 1991; Thurlow et al. 2004) deal with fora, message boards and the like only peripherally and rather dwell on well-established communicative forms like email, chat or weblogs. No wonder that even providers of such discussion platforms get occasionally confused about how to call them (cf. Münz 2002).

The roots of fora and message boards, as we know them today, can be traced back to the Usenet, a network of host computers which enables users to "post messages to newsgroups that can be read (and responded to) by anyone who has access to the system through a newsreader" (URL 3). The Usenet, which was originally set up as an experimental bulletin board system at the University of North Carolina in 1979 (Jasper 1997:12), comprises the ever-expanding newsgroups, now counting more than 20,000, hosted all over the world and covering every conceivable topic. The important difference between newsgroups, which reached their prime in the mid 1990s, and present day fora/message boards is the need of a newsreader (cf. MS Outlook Express $\odot$; NewsAgent $\odot$ etc.), a program to decipher the contents of the newsgroups (cf. Schütte 2000:150). Since the year 1996, however, web based versions of this application gained popularity, rendering the need for newsreaders obsolete. Thus, (discussion, web, internet) fora and (discussion, message) boards can be subsumed under the heading of web based applications.

But what exactly makes the difference between fora and message boards? Obviously, there are some features that they do share, such as thematic diversity and a lot of predetermined templates at users' disposal. Furthermore, visitors have to register for some fora and message boards in order to contribute or even just to read entries. Admittedly, the distinction between fora and message boards is not of a dichotomous nature. On the contrary, there are also hybrid systems which merge features of both or let users decide for one form of viewing the content or another - among them www.selfhtml.org and also TSR. Still, fora and message boards should be kept apart since they differ considerably with regard to their internal arrangements.

As can be seen in Figure 1, fora give insight into the chronological and referential order of the ongoing discussion on their first page and thus visualize the argumentative maze (cf. Münz 2002). This means that all chronologically ordered 


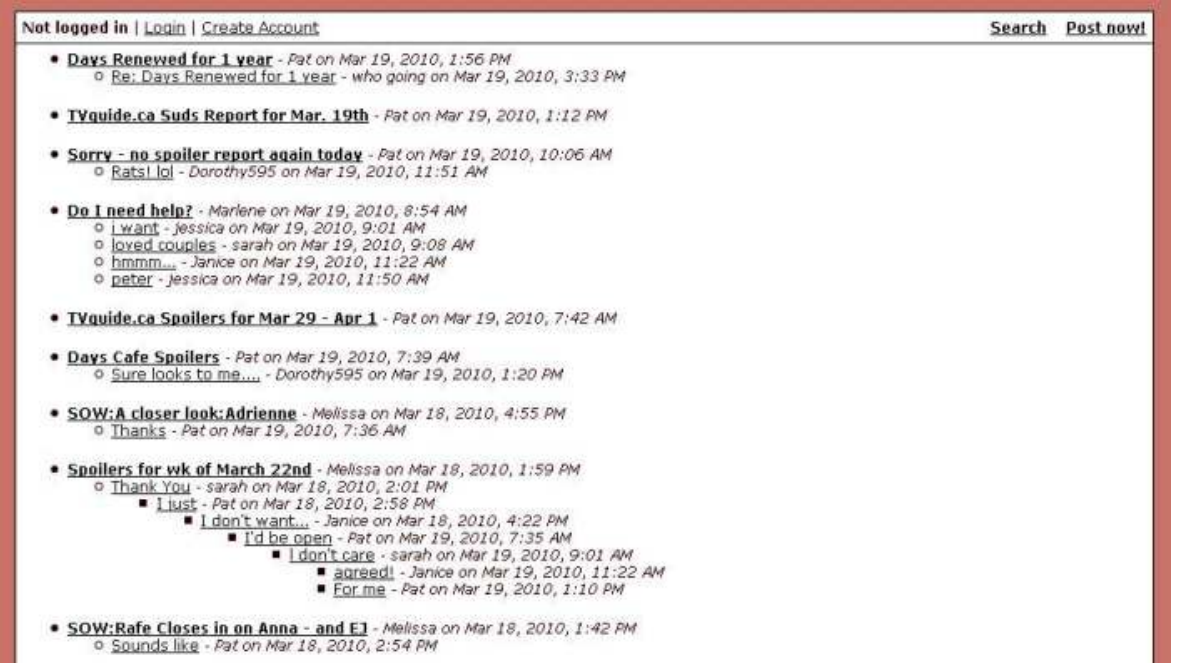

Figure 1. Structure of a typical forum homepage (URL 4)

chains of contributions pertaining to one particular topic, the so-called threads, can be perceived at once. Owing to the tree structure, a forum thus enables users to quickly establish reference and to recognize at a glance which entry refers to which. Furthermore, this schematic representation of headings (standing for complete entries) perfectly visualizes the nature of the ongoing online discussion, which can be flat at times, for example, when all users respond to one entry (see forth bullet in the upper half), or rather complex (see eighth bullet in the lower half). In the latter case, users do not only respond to the original post itself, but take comments and responses to this first post as a springboard for new contributions. To see the full text version of the entries, users just have to click on their headings within the tree structure. If one page is filled, a new one is opened automatically with new entries always posted on top of the latest page. Older contributions usually do not vanish into thin air but are meticulously stored away, as they are relegated to other pages posterior to the first page or even to an archive. One way or another, discourse produced on such pages is always retrievable, less fleeting than its FtF counterpart and thus tangible, shared history (see Sections 6.2, 6.3).

Message boards, on the other hand, are structured differently in that they offer their users several thematic categories to pick from (see Figure 2). In this mostly two-stage introductory navigation, ranging from overarching categories such as "Live Advice" or "Hobbies and Interests" to subordinate topics like "Health and Relationships" or "Fitness", users are redirected systematically in order for them to find the right place to read appealing posts or to place their own contribution. A 


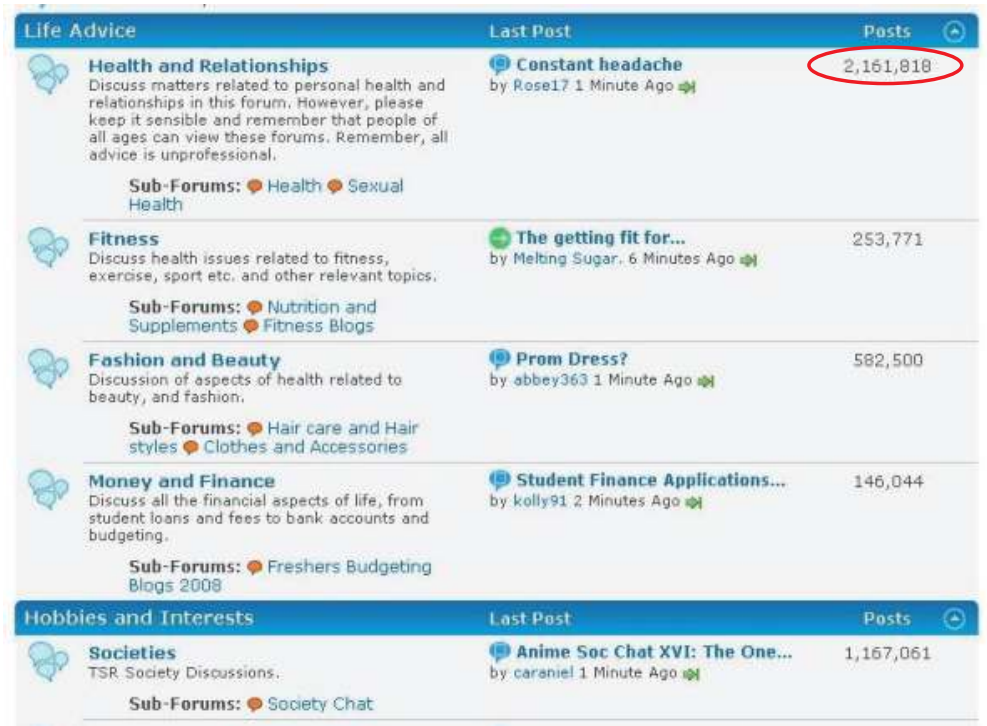

Figure 2. Structure of the homepage of the message board The Student Room (URL 5)

\begin{tabular}{|c|c|c|c|}
\hline Thread / Thread Starter & Last Post $\mathbf{V}$ & Replies & Views \\
\hline (2) (P Sticky: The Ultimate Society Guide and FAQ & $\begin{array}{l}\text { by LPK } \Rightarrow 1 \\
03-09-2007\end{array}$ & 5 & 5,952 \\
\hline$\bigoplus \bigoplus$ Sticky: TSR Societies: Index Thread & $\begin{array}{l}\text { by } L P K \Rightarrow 1 \\
02-06-2007\end{array}$ & 6 & 3,402 \\
\hline 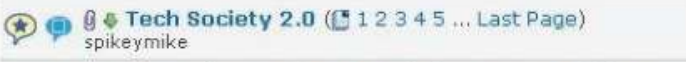 & $\begin{array}{l}\text { by alexsheppard11 } \nRightarrow \\
37 \text { Minutes Ago }\end{array}$ & 2,728 & 2,729 \\
\hline 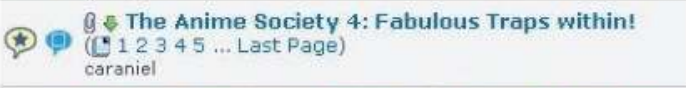 & $\begin{array}{l}\text { by Keoje } \Rightarrow 1 \\
48 \text { Minutes Ago }\end{array}$ & & \\
\hline 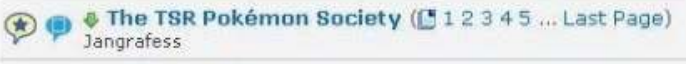 & $\begin{array}{l}\text { by paddy } \Rightarrow 1 \\
1 \text { Hour Ago }\end{array}$ & 1,582 & 6,475 \\
\hline ๑ DofE Award & $\begin{array}{l}\text { by wizard } 710 \Rightarrow \\
2 \text { Hours Ago }\end{array}$ & 5 & 6 \\
\hline
\end{tabular}

Figure 3. List of threads within a subtopic in The Student Room (URL 6)

look at this superordinate page does not only inform users of their topical choices, but also indicates "what's hot and what's not" by listing the sum total of posts so far uploaded for one topic (see circle on the top right, Figure 2). Having picked an attractive topic, users are subsequently presented with lists of topic related threads, in which each thread is represented by means of the heading of its opening post (OP), furnished with information about the last post and the number of replies and views. This is illustrated in the last two columns on the right side of Figure 3. 


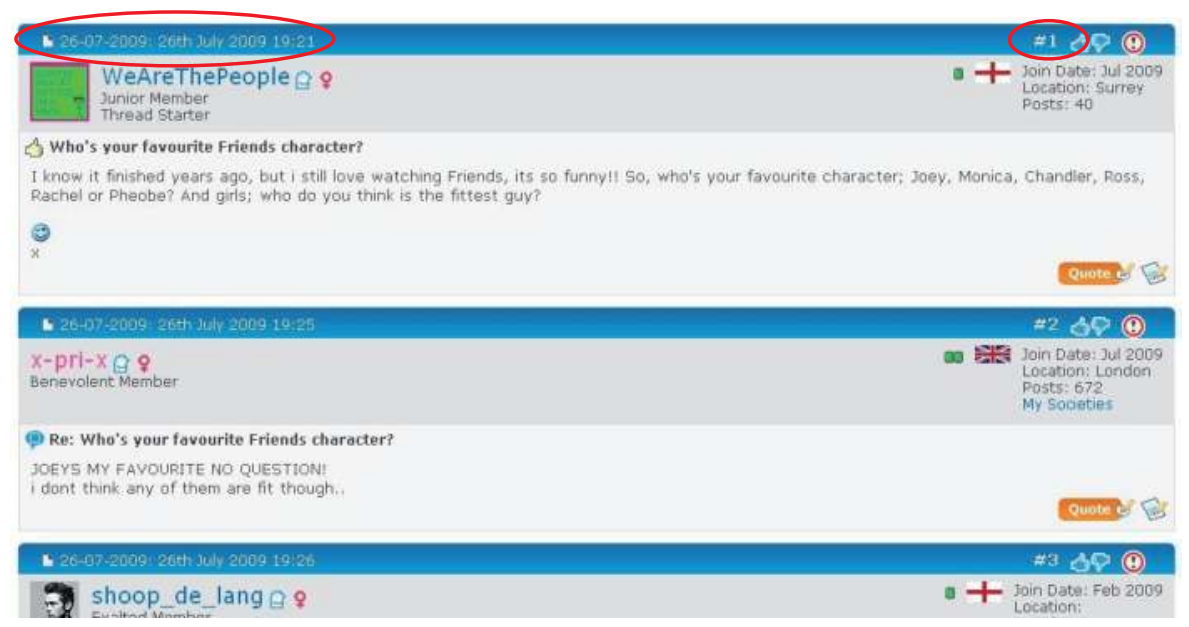

Figure 4. Beginning of a thread in the message board The Student Room (URL 7)

In order to read a complete thread, users have to click on one of the headings in the list and are then presented with the opening post and all the following contributions. All posts appear in chronological order, most of the time latest on top. The exact moment of posting is revealed through the time stamp on top of each message. For the sake of clarity, The Student Room and many other message boards also number the contributions in threads, assigning the "\#1"-tag to the thread starter and then counting upwards (see circles in Figure 4). Since this listlike layout is based on a strictly chronological order of posts, it does not reveal their referential order which is why users actually have to read entries (or at least their subject headings) to distinguish posts that give an answer to the original contribution from those which already comment on the comment. In contrast to fora, however, users do not have to click on each and every heading within a thread separately in order to read actual posts. The layout is thus less disruptive for the reading flow and encourages lively discussions within well-defined topics. In cases where discussions run the risk of going beyond the actual scope of the thread, there is always the possibility of starting a new thread.

Message boards are a highly chameleonic, multifunctional and alterable type of CMC, which is why it escapes generalizations and classifications. One common denominator, however, is the asynchronicity of communication (see above). This is the reason why users are not pressured by time constraints when composing or commenting on entries. As a consequence, discussions can be continued with or without interruptions over days, months or even years. What is more, this 
temporal freedom puts users - at least theoretically - in the comfortable position to compose and edit as well as to interpret and reflect on complex and wellwrought entries in peace and quiet (Tanskanen 2007: 89; cf. Suler 2005). Posts are basically phrased with the help of users' keyboards, although most of the message boards allow for the insertion of audio-visual elements, such as pictures, graphics, sound bites, videos, links etc. as well. Verbal input is thus complemented with stimuli for other senses, which clearly enrich communicative exchanges via message boards.

Of course, message boards such as The Student Room offer their users further technical properties, namely those which are primarily useful for the recreation of social context and the management of online interaction - mechanisms which allow for message boards to be the joyful and colorful places mushrooming all over the WWW, which have become so popular in the last few decades. Some 40 years ago, however, research did predict anything but a rosy future for $\mathrm{CMC}$ and its potential for interpersonal exchange, which is at the center of this study. Before we will return to discussing further properties of message boards, we need to answer the following important questions: Why was the social potential of CMC underestimated? Which apparently wrong assumptions affected the image of CMC in the first place? Since the forthcoming analysis of interpersonal relations sets out to present at least message boards in a more realistic light in terms of its social potential, we need to know about the former reservations against CMC. Only that way can we detect the logical and analytical mistakes of the past.

\subsection{Reservations against the social potential of CMC}

Despite the promising developments of the technical innovations described above, not every detail of the new communicative potential of CMC was appreciated and welcomed with open arms immediately. Especially its usage for social purposes was highly doubted at first so that critical voices rose immediately, warning against the alleged risks of using computer networks for interpersonal communication. This skepticism was fueled by two major lines of concern which, although definitely overstating the case, will be outlined in the following in more detail: the fear of an exuberant and inevitable influence of technology on (inter)personal cognitions as formulated in the theory of technological determinism and the alleged weaknesses of CMC itself, which were assumed to be medially inherent and which found expression in the so-called cues-filtered-out approaches. 


\subsubsection{Technological determinism vs. social constructionism}

The skepticism about the use of computer networks for interpersonal communication is reflected in an overly negative sentiment towards technology called technological determinism. This theory, which owes its name to the American sociologist and economist Veblen, takes up an extreme position in explaining the relationship between technological change and social life:

[P]articular technical developments, communications technologies or media, or, most broadly, technology in general are the sole or prime antecedent causes of changes in society, and technology is seen as the fundamental condition underlying the pattern of social organization.

(Chandler 1995: n.p.)

Technology is therefore regarded as one of the most powerful forces behind social change "at every level including institutions, social interaction and individuals" (ibid.). Human factors and social conventions, on the other hand, are erroneously dismissed as secondary phenomena. The inner makeup of technological determinism is thus considered to be extremely influential insofar as it is

- reductionistic - reducing the relationship between technology and cultures to one of straightforward cause and effect;

- monistic - oversimplifying an otherwise complex relationship to the effects of a single factor (rather than being multi-causal);

- neutralizing - representing technology as neutral or value-free and therefore absolved of 'responsibility';

- technological imperative - presenting technological 'progress' as unstoppable, inevitable, and irreversible.

(Thurlow et al. 2004: 41 following Chandler 1995)

A special subset of technological determinism is media determinism, its quintessence being encapsulated in McLuhan's famous aphorism "the medium is the message" (1964). Although he does not explain the mechanisms through which the widespread use of electronic media brings about social change, McLuhan supports the media-deterministic view insofar as he analyzes media as extensions of the human senses or processes. As a result of media use, the organization of human senses and their consciousness - in McLuhan's words, people's "sensory balance" - are altered (cf. Meyrowitz 1985:3, 17).

As the controversy is sparked off by the question how far technology does indeed condition social change, Chandler (1995) justifiably cautions against overgeneralizations which are based on "the nature and pitfalls of a very persuasive 
stance". ${ }^{10}$ Subsequently, technological determinism has been reviewed more and more critically by scholars in recent time and has been contrasted with yet another extreme view: appropriation theory or social constructionism. Although these moderated approaches do not view users as operating in ways which are downright dictated by technology, they do not mirror reality either. These two approaches embody a stark contrast to technology-led theories of social change since they reverse the roles of cause and effect, purporting that technological innovations are solely the (secondary) condensates of social constructs. Accordingly, users are reinstituted as masters of the situation as they are the ones to consciously "pick and choose from what is available, at times using things in unexpected ways, at times not using some of the possibilities" (Baym 1995: 150). At least in contrast to technological determinism, this perspective has been proven to be a more realistic assessment of online interaction by several studies, as, for example, by Baym's (1995) work on online soap opera communities, which comes to the conclusion that "even the most mundane interactions require that people draw upon pre-existing resources that have meaning within a community to create and invoke event types, identities, relationships, and norms" (1995: 150).

The truth most certainly lies somewhere between these two extreme positions: Certainly, we cannot act on the assumption that the "medium itself is [only] a neutral delivery system" (Meyrowitz 1985:15), which is why medium theorists "suggest that media are not simply channels for conveying information between two or more environments, but rather environments in and of themselves" (Meyrowitz 1985: 16). Although the means of communication do not shape culture and personality completely, social and technological factors are still mutually dependent. For that reason, the medium, or rather the communicative platform, cannot be overlooked as a factor in analyses any longer (cf. Meyrowitz 1985: 18).

\subsubsection{Cues-filtered-out approaches}

The second strand of reservations according to which CMC is ill-suited for interpersonal exchange, let alone social interaction, when compared to FtF get-togethers points directly to the nature of CMC itself. In the late 1970s until the mid 1980s, early scholars of CMC such as Baron (1984) and Kiesler et al. (1984)

10. Chandler (1995) adds that the antagonism described here is known in economics as a technology-push theory vs. a demand-pull theory. A similar controversy can be witnessed in developmental psychology over nature versus nurture which is one between genetic and environmental determinism. While Thomas Hobbes was an early advocate of the importance of nature (heredity), Jean-Jacques Rousseau represents the most famous proponent of the importance of nurture (or experience) (cf. Chandler 1995). 
identified a number of characteristics inherent in text-based CMC which they considered to be detrimental for the creation and maintenance of social bonds online. They held that

[e]lectronic media do not efficiently communicate nuances of meaning and frame of mind, organizational loyalties, symbolic procedural variations, and, especially, individuating details about people that might be embodied in their dress, location, demeanor, and expressiveness.

(Kiesler et al. 1984: 1126)

Apparently, CMC lacked important qualities of FtF communication, making it an inadequate means for social interaction. This stance finds expression in the so-called deficit approaches or cues-filtered-out approaches, coined by Culnan/ Markus in 1987, the trademark of which being long successions of judgmental adjectives. According to Collins (1992), social context cues, which are supposed to be "filtered out" in said approaches, refer to various geographic, organizational, and situational variables that have ample influence not only on how conversational content is conceived, but also on how it is perceived by users. These can include demographic variables such as age, gender, race, socio-economic status, residence as well as personal characteristics such as appearance, dress, accent, mood, size, and attitude, but also interactional or back-channeling cues as conveyed by eyecontact, facial expression, tone of voice, and body language. All these para- and nonverbal cues are instantly present in $\mathrm{FtF}$ communication and generally rich in relational information. In CMC, however, they are seemingly absent, which results in insalubrious effects concerning interpersonal relations, as some information cannot be fully transferred (McKenna/Bargh 2000). For this reason, ambiguities, for example concerning the "intended" emotion, can arise. Following Derks et al. (2008:777), these misinterpretations can result in inappropriate reactions on the part of the hearer, escalation of conflicts or negative judgments of the other user.

In fact, the notion cues-filtered-out approaches is an umbrella term for three individual, though rather similar approaches, all of which proclaim the same (negative) view on the social capacities of CMC to be shortly summarized in the following:

\section{Social presence theory}

This theory ${ }^{11}$ holds a connection between the absence of social context cues and the presence of uninhibited verbal behavior in CMC (cf. Kiesler et al. 1984). This

11. Although not designed to explain CMC in the first place and "at best a vague concept, never clearly defined by its proponents" (Svenning/Ruchinskas 1984:248), the theory has nevertheless been widely accepted (and later also criticized) as a major theory in this area (cf. Walther 1992:55). 
so-called disinhibition effect is owed to the fact that audiences are not present physically, depriving users of the possibility to detect social context cues in their interlocutors. Baym (1995: 139f.) concludes that, "[w]ith these cues to social context removed, the discourse is left in a social vacuum quite different from faceto-face interaction". CMC is thus extremely "low in social presence" (Short et al. 1976) and users can only imagine their interlocutors while typing seemingly for and by themselves, which is why their attentional focus shifts from actual audiences to the act of composing and responding to messages alone (Siegel et al. 1986). Accordingly, the users' "level of sympathetic involvement with others is attenuated and people don't need to be sensitive to other's [sic] feelings or messages, nor do they need to avoid impoliteness" (Collins 1992:n.p.). The absence of a discernable audience entails yet another consequence: Since "the fear of physical retaliation as sanction for verbal aggression is non-existent" (ibid.), a sense of freedom arises. The only remedy available in CMC is the showing of more nonconforming, direct, or even aggressive behavior in return - unrestrained conduct also known as flaming (Avgerinakou 2003:273f., cf. Section 5.7).

\section{De-individuation theory}

The second theory focuses on the self-perception of the producing user. Thurlow et al. (2004:63) report that de-individuation "entails the subjugation to the group and a concomitant reduction in self-focus [as] the group activity simply becomes more important". Since users hardly emit any social cues themselves, feelings of technologically supported social anonymity and reduced self-awareness arise. Spears/Lea (1994) suggested that this kind of anonymity within a powerful audience may have a liberating effect, thus reducing inhibitions and feelings of accountability to the audience as well as fostering self-disclosure. Accordingly, feelings of embarrassment, guilt, empathy, and fear of retribution and rejection are generally reduced; while antinormative, unrestrained, and uninhibited behavior becomes more salient (Siegel et al. 1986:161). The pressure to conform to norms and expectations of the group is thus lifted from the anonymous communicator's shoulders (cf. Douglas/McGarty 2001:400).

Note, however, that anonymity should not be equaled with not being identifiable (Joinson 1998). Although visual anonymity may be a given in the context of $\mathrm{CMC}$, as interlocutors literally cannot be seen, users are still identifiable (depending on the particular CMC platform for example by means of nicknames, email addresses, links to personal web pages etc.) and can sometimes even be linked to their true identities.

\section{Media richness theory}

Although based on the same assumption as social presence theory, the last of the three theories draws different conclusions: Scholars back then considered CMC 
a lean form of communication, attesting it a reduced capacity to transmit multiple social context cues and assessing it as being inferior for the communication of rich information. FtF, on the contrary, was held to be the richest medium of communication, "given the multiplicity of channels available to it" (Avgerinakou 2003:274). Owed to the lack of aural and visual feedback, they reckoned the general coordination of interaction, the resolving of disagreements or the reaching of a consensus to be more difficult online (cf. Hiltz et al. 1986). Accordingly, $\mathrm{CMC}$ seemed more liable than FtF to cause social friction. Consequently, "CMC is deemed appropriate for the transfer of simple or unequivocal messages, but insufficient for the communication of more equivocal, ambiguous, or emotional information" (ibid.). Being discredited as impoverished and fragmented form of communication (cf. Herring 2001:613), CMC was considered an inadequate mode of interpersonal communication (cf. reviews by Culnan/Markus 1987; Walther 1996; Avgerinakou 2003).

To sum it all up, at the beginning of the 1980s, CMC stood accused of being asocial, emotionally cold, impersonal, anonymous and unfriendly (Lea/Spears 1995:214), in short: "the quality of communication is reduced as a result of the technological restraints of the Internet" (Thurlow et al. 2004:46, original emphasis). Above that, CMC was also called antisocial as "it has a negative impact on offline communication and offline relationships" (ibid.) because people cut themselves off from "real" FtF relationships. ${ }^{12}$ Then again, interpersonal relationships, which were established solely online, were shrugged off as being "superficial, unstable, shallow, and ephemeral leading to the creation of the specter of pseudocommunity" (Lee 2005: 387 with reference to Foster 1997; Gimenez 1997). Defeatist evaluations of CMC as displayed in these quotes should not be left uncommented, though. The next chapter will demonstrate that most of the findings of early CMC scholars should be handled with extreme care, to say the least.

\subsection{Everything is not lost: The discovery of social potential within CMC}

Despite the negative judgment of CMC passed through their research, Kiesler et al. (1984:1130) are the first to admit "that [their] own data do not provide any evidence to distinguish among these tentative and somewhat limited potential explanations", a statement which Culnan/Markus (1987:430) believe to apply to

12. A survey by Katz et al. (2001:412) proved this claim to be wrong: It showed that the more time Internet users spent online, the more likely they were to belong to offline religious, leisure, and community organizations, when compared to nonusers. Internet use is thus not responsible for the weakening of the fabric of neighborhoods and communities. 
their entire body of work. In explaining that the deficit approaches "were mostly developed at a time when CMC hadn't really begun to take off yet - either in terms of popularity or academic research", Thurlow et al. (2004:60) come to the rescue of those scholars involved in early CMC research. Ten years after the launching of the first experimental studies of the early 1980s, several field studies investigating CMC could not find the evidence to uphold the unsocial view of CMC (Walther 1992: 53). First and foremost, works by Walther and colleagues (e.g. Walther 1992; Walther/Burgoon 1992; Walther et al. 1994) have revealed that the differences between $\mathrm{CMC}$ and FtF interaction are not that large and, even more importantly, tend to dissolve over time. The social potential of CMC thus needs to be put into perspective.

\subsubsection{Opposed findings of more recent approaches}

Owing to more recent research, mostly conducted as field, not as laboratory studies $^{13}$ (e.g. Feldman 1987; Ord 1989; Weedman 1991), the old allegations towards CMC could be successfully refuted as popular myths, which only a few lay people and journalists regard as valid to this very day (Thurlow et al. 2004: 47, 70; cf. URL $1,2)$. Instead, a more realistic assessment of today's situation has been revealed, sometimes even furthering diametrically opposed findings. As a matter of a fact, the rather distorted picture drawn up for CMC was partly due to the fact that cues-filtered-out approaches operated on several false assumptions. Looking into and hopefully overcoming these methodological mistakes, more recent studies thus try

- to work on the problem empirically;

- not to treat FtF communication as the optimal form of communication and thus as the standard against which to measure CMC: As Thurlow et al. (2004:49) fittingly remark, "[y]ou just have to think about a really boring class you've attended to know that [sic!] FtF, bodily presence is no guarantee of warm, personal, or sociable communication!";

- to distinguish more carefully among different forms of communication within $\mathrm{CMC}$ (synchronous vs. asynchronous, private vs. public vs. semi-public etc.);

13. As summarized by Taylor (2000: 98f.), laboratory studies used artificial designs and procedures: simulations comprised discussion groups of unnaturally small sizes, limited discussions to only a few minutes, appointed participants who were unaccustomed to using CMC and gave them very narrow tasks to perform. The validity of those results should in no event be generalized. 
- to distinguish more carefully among different types of tasks being worked on;

- to distinguish more carefully among different users, their relationships and their personalities;

- to determine the extent and nature of flaming as carefully as possible (see Section 5.7): In surveying articles on CMC extensively, Lea et al. (1992) proved that studies counting instances of flaming in CMC were not at all comparable with each other, let alone with findings for FtF. Proceeding from different notions of flaming, they operationalized this hazy phenomenon inconsistently (e.g. language which is either extremely positive or negative (McCormick/ McCormick 1992), swearing and insults (Siegel et al. 1986) or even all messages conveying bad news etc. (Sproull/Kiesler 1986);

- not to treat flaming as a decontextualized phenomenon but consider the social context that is predefined or communicated via the medium;

- to collect user perceptions of each other and of the group when dealing with intrapersonal and interpersonal processes (Taylor 2000: 98);

- to include time as a crucial factor for the development of compensatory mechanisms to make up for the absence of social context cues.

Especially the last point on the list deserves a little more attention: Since fewer social cues can be transported within one message, we cannot proceed from the same time frame as in FtF communication for the establishment of interpersonal relations. In time, however, users can and do develop a system which provides them, to a certain degree, with compensatory mechanisms such as emoticons, smileys, and capitalizations to emulate FtF contextual cues (cf. Avgerinakou 2003:275) and to make up for the "dramaturgical weakness of electronic media" (Kiesler et al. 1984:1125). Even back in the 1980s, Kiesler et al. considered it the task of future research to "discover how groups respond to the difference [between CMC and FtF communication]; how, given time, groups work out new communication traditions and rules" (1984:1127). As one of those future researchers, Walther and his associates came to the following conclusion:

communicators using any medium experience the similar needs for uncertainty reduction and affinity, and to meet these needs CMC users will adapt their linguistic contextual behaviors to these solicitation and presentation of socially revealing, relational behavior. The critical difference between FtF and CMC from this perspective is a question of rate, not capability.

(Walther et al. 1994:465)

Obviously, users act on the same prerequisites online than they do in FtF settings, as they want to be liked and connect with people (cf. Section 4.3). They need, however, comparatively more time not only to reach decisions but more importantly 
also to convey social cues, which are necessary for the establishment and/or negotiation of relationships. Literally slowing down the communicative process are the typing requirements, to give but one example.

Admittedly, the lack of channels is indeed a disadvantage of CMC - none that cannot be compensated for, though. Users simply need to adapt their behavior in putting their otherwise nonverbal behavior on record, thus learning new ways of verbalizing relational content (cf. Walther/Burgoon 1992). As time limitations were common in earlier (mainly task-oriented) laboratory studies investigating $\mathrm{CMC}$, we are now presented with the variable that may have had major influence on the negative outcome in terms of the social potential of CMC. As a matter of fact, slower moving CMC interaction may have been cut off before sufficient messages were exchanged for the accruement of interpersonal effects (Walther et al. 1994:465). Although studies to prove this new perspective, other than anecdotal or case study analyses, are still far and few between, it is safe to assume that researchers should focus on longitudinal rather than cross-sectional studies in order to yield suitable results for the investigation of interpersonal and social patterns.

\subsubsection{Compensatory mechanisms and netlingo}

Instead of replying to a statement, you can smirk. Instead of leaving the room, you can disappear in a cloud of iridescent, bubble-gum-flavored bubbles.

(Rheingold 1993: 148)

As the previous chapters have proven, $\mathrm{FtF}$ interaction has many qualities that are absent in CMC settings, most importantly ever present and directly accessible social context cues. These are imperative for interpersonal interaction insofar as they do not only help to regulate the interaction (among others also by conveying emotions) but above that also to provide speakers with valuable information about their interlocutors, which certainly promote the formation of impressions and the more accurate evaluation of their behavior (Culnan/Markus 1987:427). Fortunately, CMC does not have to forfeit these two major functions of social context cues, since they are substituted - at least to a certain degree - by online compensatory mechanisms: new electronic forms of communication feature characteristics and functions which do not even have an equivalent in FtF but are nevertheless quite beneficial for online interaction. This assessment is backed up by Walther (1992:77), who holds that "equilibrium theoretic principles support the contention of cue substitutability, in that interactants are likely to adopt other symbol systems to convey affective messages that are unavailable nonverbally". Walther/Parks (2002) call this new perception of CMC the cues-filtered-in 
approach. Above that, seasoned users are extremely resourceful and become adept to using and interpreting creative and sometimes even playful textual signs that are supposed to make up for the felt lack of "real" para- and nonverbal cues. It takes, of course, some time to establish these new conventions, which are even deepened and further entrenched by the feeling of connectedness and relational commitment through a history of previous and the anticipation of future interaction. To be fair, one should mention that Hiltz/Turoff (1981) as well as Short et al. (1976) foresaw this development even as early as the late 1970s, which only hints to the very natural phenomenon of human beings always trying to make the best of situations and easily accommodate to new (communicative) surroundings.

These "keyboard tricks" (Lea/Spears 1995:217), also known as "affection and metacommunicative cues" (Walther 1992: 80) or "relational icons" (Asteroff 1987) have become very common in usage and enjoy great popularity. Their most prominent representative surely is the emoticon (a blend of emotion and icon), also known as smiley :-), sometimes also winky;-) or frowny:-(, which is basically a combination of punctuation marks and other ASCII ${ }^{14}$ characters (for example @ >--;-- "a rose"). When read sideways, ${ }^{15}$ an emoticon resembles a face (or any other iconic representation for that matter) which reveals information about the author's current mood or mental state (Constantin et al. 2002) and "the intended emotional tone of a message" (Lea/Spears 1995:217; cf. Crystal 2001). Depending on the technical equipment of the type of CMC, users have selections of more or less elaborate, sometimes even animated ready-made smileys at their disposal, which can be inserted into the message with one click. ${ }^{16}$

In order to avoid ambiguity about how a message is supposed to be understood, users can always resort to making their emotions explicit by spelling them out ("I am angry!, This is scary!, I would like to hit you!") (Derks et al. 2008: 768, 777). If there is still a need for more information, acronyms can be of some help in establishing clarity: Typing MORF ("male or female"), GOS ("gay or straight"), or $A / S / L$ ("age, sex, location") is a simple way of inquiring basic demographic information about interlocutors.

Other ways of disclosing emotional states include using asterisks to stress certain items in the text $\left({ }^{*} \operatorname{grin}{ }^{*}\right)$ or capitalization to indicate screaming and thus

14. ASCII is short for the American Standard Code for Information Interchange, referring to all characters typically available on a standard keyboard.

15. This reading technique is not necessary for the Asian equivalent of smileys, the so-called kawaicons, such as $\left(\wedge^{\left.\mathbf{O}^{\wedge}\right)}\right.$ or $\backslash\left(\wedge^{-} \wedge\right) /$ as expressions of joy.

16. The multitude of smileys available in The Student Room (TSR) can be viewed under URL 8 . 
hinting at a rather emphatic way of conveying a message. Therefore, capitalization can be seen as an indicator for impatience, frustration or maybe even anger. Emotions can also be put across rather subtly by changing the font, color and/or size of one's words. Another popular way of expressing oneself online the quickest possible way is by using the aforementioned acronyms. They are multifunctional in that they are not only used to convey emotions ( $g$ "grin", lol "laughing out loud", rofl "rolling on floor laughing" etc.), but also to get across reoccurring routine formulae, sometimes concerning the circumstances of the (invisible) production process ( $b r b$ "be right back", $b b l$ "be back later", imho "in my humble opinion", $w d y t$ "what do you think?", $c y$ "see you", thx "thanks", irl "in real life", F2F "faceto-face", some1 "someone" etc.) (Thurlow et al. 2004: 125). This latter function can also be implemented by so-called actions or emotes, which are short phrases such as $*\{$ Sender $\}$ eyes you up and down ${ }^{*} *\{$ Sender $\}$ cries on your shoulder $*$ ibid. 125). According to Benwell/Stokoe (2006:251) they are "crucial ways of contributing to identity work online and compensating for an absence of visual context, since they can express relationships, flesh out dramaturgical space, reveal inner states or motivations, or imbue the domain with a surreal cartoonish quality".

As a matter of fact, we can learn a great deal about our participants just by looking at their language, meaning their vocabulary, their grammar, their style (Thurlow et al. 2004:53). A lot of the following typographic gimmicks try to imitate prosody, which is crucial not only for smooth online interaction, as prosody is an important element for the interpretation of speaker intent. Electronic paraverbal cues are, among others, transported through intentional misspelling (for example the repetition of a vowel to emulate drawn-out pronunciation, as in coooool or he is soooo cuuuuute, or of a final consonant to indicate stress, as in yearsssssss ago). According to Walther (1992: 80), "lexical surrogates for vocal segregates" add affective information and further convey informality, as for example hmmm or yuk yuk. Spatial arrays are used to indicate pauses or to highlight words or phrases. The opposite, typing words without spaces in between, can be a sign of increased tempo. Grammatical markers, such as exclamation or question marks as well as periods, can also be used as a means of highlighting, when used repeatedly. Most of all, rows of periods have become a classic means for indicating ellipsis of some sort and have even found their way to other communicative arenas (Walther 1992: 79f.). In terms of creativity, only the sky is the limit.

In exploiting the system's features, language is a "powerful strategic resource indeed the primary resource - for the creation of social reality in text-based CMC" (Herring 2001:623). For this reason, research has explored this special online language in depth which goes by a multitude of names such as weblish, 
netlingo, e-talk, tech-speak, wired-style, geek-speak ${ }^{17}$ and netspeak. Despite these extravagant but harmless names, we should not assume that netlingo, to pick one such umbrella term, is a completely different language, which radically affects and even destroys "proper" language (cf. Baron 1984: 131). Early assessments that "[n]etspeak is a development of millennial significance [and a] new medium of linguistic communication" as enthusiastically proclaimed by Crystal (2001:238f.), clearly overstate the case. The only truly distinctive feature of netlingo is its invincible amount of creativity, which clearly surpasses "normal" language. Surely, no other "language" exhibits so many ad hoc word formation processes such as "weblish, shareware, netiquette, e- and cyber-anything" (Thurlow et al. 2004: 124) than netlingo. There is thus a "strong tendency to break traditional conventions of written language to help create a more social orientation of speech" (ibid. 125) which - depending on a wide range of contextual variables such as type of CMC, participants, topic and purpose - leads to a hybrid form of speech and writing. ${ }^{18}$

This compilation of compensatory mechanisms created by users for the expression of social context cues and emotions clearly disproves the claim that CMC is per se impersonal and emotionless. Still, we need to keep in mind when interpreting emoticons and the like that they are more deliberate and voluntary in comparison to their rather spontaneous FtF counterparts. One could therefore say that users of CMC have more control over the expression of their emotions, which is partly due to the fact that there is a time-lag between receiving and answering a message. Theoretically at least, this allows users to choose their words and emoticons carefully. Derks et al. (2008:778) therefore object that the "use of emoticons $[\ldots]$ does not necessarily tell us that individuals experience an emotion, as it only conveys the conscious intentions and motives of the person using the emoticon". Whether or not these cues are comparable with their FtF counterparts in terms of subtlety and range of expression is yet another kettle of fish that has been dealt with repeatedly (see Lea/Spears 1995:216). Graham (2008:285) warns that

[s]mileys $[\ldots]$ are not adequate to approximate the range of paralinguistic markers that help clarify intent in a face-to-face setting. This doesn't mean that hearers can't and don't assign intent to speakers, but simply the lack of adequate tools to approximate paralinguistic and non-verbal messages may complicate this process.

17. This term denotes the jargon of computer "geeks" and other specialists who are heavily involved in developing communication technologies.

18. For a detailed discussion of the meanwhile "classic issue" of the fusion of orality and literacy in CMC, see Murray (1988), Beißwenger (2002), Dürscheid (1999, 2003, 2006), Schütte (2000, 2002), Storrer (2001), Stein (2005) and Herring (2007). 
Whatever the outcome of this discussion, it is beyond question that emulated social context cues nevertheless fill the virtual space with more warmth and socio-emotional content than was expected for a long time. Several studies have proven this claim (Rice/Love 1987; Ord 1989; McCormick/McCormick 1992; Rheingold 1993) and others identified many ways in which emotions and impressions are conveyed in this textual form of communication (e.g. Ferrara et al. 1991; Selfe/Meyer 1991; Wilkins 1991). Thus, social presence can indeed "be cultured" (Johansen et al. 1988: 141).

The question which needs to be answered yet is this: Which exactly are the inherent, predetermined mechanisms of message boards such as The Student Room which help its users emulate social context cues and manage their shared interaction? In answering that question we have to focus on various templates, an integral structural feature of message boards which either allow users to chose from a drop-down menu or to fill in information totally freely and independent of default settings. Therefore, a user's leeway for creativeness is in a way pre-determined by the nature of the respective template (see Figure 9 below).

The first impression of one's (presumably unknown) interlocutor is formed through his username, the so-called nickname and his avatar, both of which can be picked freely by the user. TSR itself provides a huge amount of ready-made avatars for its users to serve as a digital "actor". While the avatar is an optional feature, users are asked to choose a nickname in the process of registration in order for them to be identifiable (see Figure 5). When clicking on the nickname (or on the avatar) one is given the option "View Public Profile", which may contain more personal information. If the member bothers to fill the gaps, other users can learn about his/her name, gender, age, orientation, bio(graphy), academic standing, interests etc. In some cases, the option "Visit X's homepage!" redirects the interested reader to that person's webpage, which can be on facebook or twitter or any other (social network) site as well (see Figure 6).

This proves that identifiability is indeed a matter of degree and strongly dependent on the user's willingness to share personal data. Above that, this menu

\begin{tabular}{|c|c|c|}
\hline \multicolumn{3}{|c|}{ Q2. Days Ago: 18 th March 201022,06} \\
\hline & $\begin{array}{l}\text { No.1Schadenfreude } \Theta \sigma^{\top 7} \\
\text { New Member } \\
\text { Thread Starter }\end{array}$ & $\begin{array}{l}\text { Join Date: Mar } 2010 \\
\text { Location: London } \\
\text { Posts: } 3\end{array}$ \\
\hline \multicolumn{3}{|c|}{ I I suppose I should introduce myself. } \\
\hline \multicolumn{3}{|c|}{$\begin{array}{l}\text { Hi my name is (insert real name here but I've already } \\
\text { deemed that irrelevant since I have a username and most } \\
\text { likely nobody would remember it.) }\end{array}$} \\
\hline I look & ward to my stay here. & \\
\hline
\end{tabular}

Figure 5. Standard TSR header of posts to identify a user (left side) 


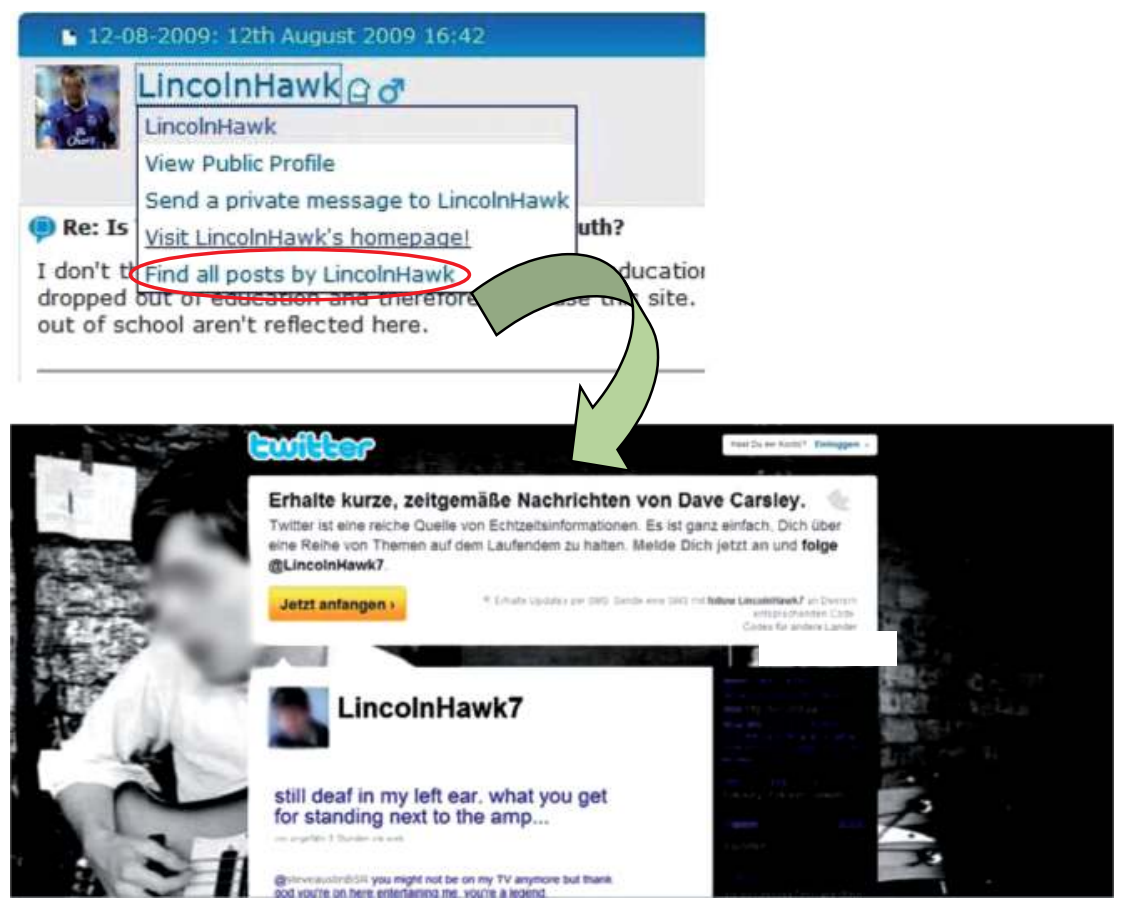

Figure 6. User's homepage on twitter, redirected from the drop-down menu

allows to contact users directly via PM (private message) or via email and to search the entire board for all contributions ever posted by that specific user.

Underneath each nickname is a label (or status title), providing information about the member's status within the group. According to his number of posts and activity, this label can read "New Member", "Adored and Respected Member" or even "Vengeful, Imperial Overlord of The Student Room", among many others. If a user is the one to start a whole new thread, this is indicated through the additional label "thread starter". In TSR, small icons reveal the (maybe feigned) gender of the member as well as his momentary status (online or offline). The statistics on the upper right hand side (see Figure 7) further hint at the joining date, the location and the number of posts. The location is, however, neither an obligatory piece of information, nor necessarily a truthful mirror of actual facts. In some cases, it only bears witness to a member's vivacious imagination. The same holds true for yet another bit of optional information: flags to signal nationality.

Some headers, as the one in Figure 7, exhibit yet another piece of supplementary information: If a user wants to show that he is a member of a society, he can do so with the help of the label "My Societies" below the count of the posts. One click on this label reveals the name of the society or societies, such as "Bon Jovi 


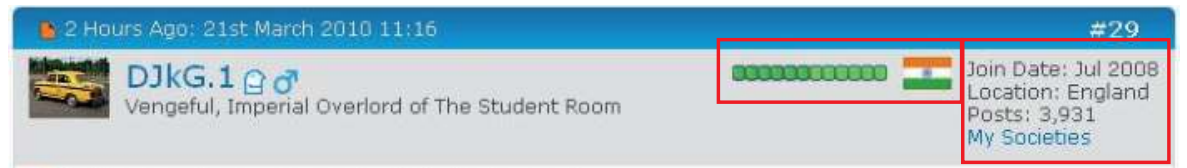

(1) Re: Do you call your parents by their first names?

I don't but my friend calls his dad Dave. Apparently it started off as a joke when he was young and they all got used to it lol.

Figure 7. Standard TSR header of posts to identify a user (right side)

Society", "Arsenal F.C. Society" or "Chemistry Society". In some cases, even information about contacting the member via skype or MSN is positioned here.

An integral part of every header is the reputation system, which can be found on the left side of the flag. While green gems basically are representative of a good reputation, red ones attest to failures in behavior - both "handed out" by ordinary members evaluating each other's behavior. A mouse over even verbalizes the number of gems. Thus we learn that the member in our example "DJkG.1 has more reputation than sense" with twelve green gems. Five red buttons, on the contrary, warn that " $\mathrm{X}$ is infamous around these parts". The number of points depends on the gravity of the offence and can be removed after a while, if the member redeems himself. In order to preserve the expressiveness of that system, it is firmly forbidden to "beg for rep" or to "gang up to give rep" (URL 8). A related system with the same purpose, the "warnings", can be used by moderators alone in order to maintain a high standard. When reaching 15 warning points, members will be banned, i.e. excluded from participating in the board, for a period of time.

The last option to further personalize a post is individualized signatures. According to Kollock/Smith "a combination of business card and bumper stickers that members use to display their interests, opinions, and occupations" (1999: 10), signatures can be inserted in every post and appear underneath the entry. Users can exploit this template creatively, for example by communicating further references to their personal backgrounds by listing exam dates, including pictures, cartoons, or even their favorite quotes or video clips. Figure 8 provides a very short selection. As a matter of fact, signatures may even contain instructions for potential interlocutors such as "If you're talking to me or replying to something I’ve said, please quote or I probably won't notice". Quoting, i.e. clicking on the "quote"-button to explicitly reference to previous entries, secures smooth, coherent interaction. This allows for multiple users to converse with each other in several separate conversational strands within one and the same thread. As contributions 


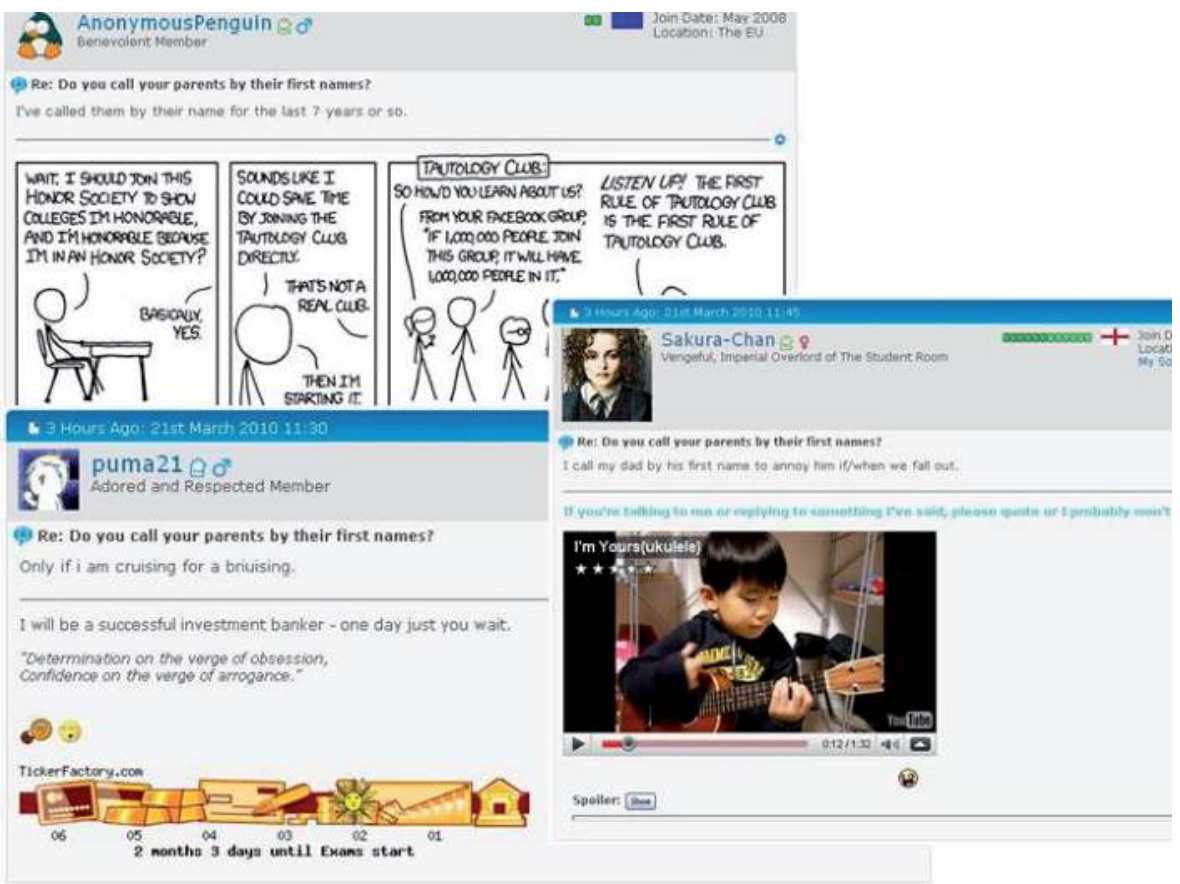

Figure 8. Selection of signatures in TSR

are posted according to their time stamps, meaning chronologically and not thematically, ensuing disrupted adjacency can thus be repaired.

As has become obvious so far, there are plenty of ways - not only in TSR to re-create and learn about members' personal attributes and status within the group as well as to organize talk. ${ }^{19}$ Although real life personalities may be kept a secret (see Section 6.2.1), compensatory mechanisms do have the power to create (divergent) life-like online personalities, which are interrelated with one another just as people in real life. TSR's buddy lists ${ }^{20}$ bear witness to the net of interpersonal relations forged among message board users. It further indicates, whether friends are online and enables users to send private messages to (multiple) members.

19. Note that all the functions described here can be accessed without being a registered member of TSR. Thus, even random lurkers (or analyzing scientists for that matter) can keep track of threads without ever getting actively involved by contributing to it. In the context of CMC, lurking is an acknowledged fact which is why users are usually aware of their posting to multiple audiences, including lurkers (see Section 3.3.1).

20. The opposite measure, ignore lists, can be used to block out posts from users who have fallen into disgrace for whatever reason. 


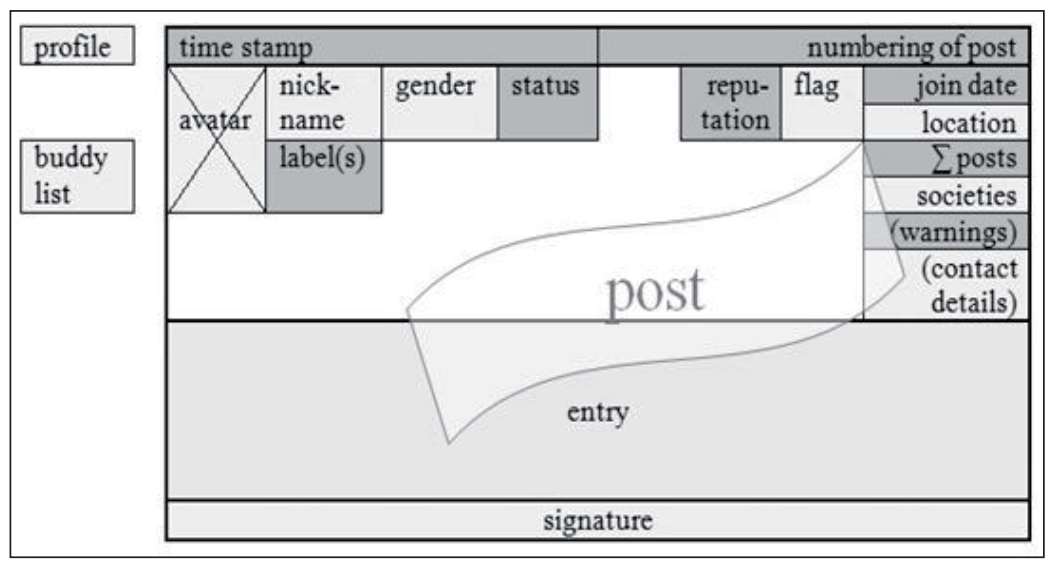

Figure 9. Prototypical TSR post with integral and accompanying components

As this feature can contain private and intimate information about users, it can only be viewed and accessed when registered to TSR.

Based on the multitude of features and templates discussed in this section, we can now draw up a blueprint of a prototypical TSR post and its components (see Figure 9). As indicated by the differing shadings, TRS components which are integral to every single post as well as those which accompany it (i.e. profiles and buddy lists) vary in terms of users' influence on their filling: While some of them are filled automatically by the system alone (dark grey squares), others are replenished by users themselves when constituting their message board account (light grey areas). Note, however, that this happens before the actual process of posting. Still choices made in this process become visible repeatedly with each and every post to come. The notion post therefore encompasses not only the actual entry with the current, up-to-date, user-specific communicative content but also all the other integral templates.

\subsection{Summary: Why CMC can be social after all}

Over the past two decades, the general opinion of CMC has definitely changed for the better, either by disproving former claims or by recognizing a positive side effect in (formerly purely disadvantageous) features of CMC. In stark contrast to former views, CMC is now regarded as a promoter for "new, and even better, social relationships, with people communicating across geographical and social boundaries and creating new friendships and communities based on their shared 
interests and concerns" (Thurlow et al. 2004:46). Accordingly, anyone can participate freely in open, democratic exchanges (Edinger/Patterson 1983; Herring 2001:620), since a lot of hierarchical dominance and power information are hidden. Although this is certainly true for real-life status cues, the following chapter will show that $\mathrm{CMC}$ is not at all free of social hierarchies either, as new structures develop over time. This supposedly beneficial aspect of CMC thus needs to be qualified instantly.

Another case of revaluation can be found in O'Sullivan's (2000) view of the "leanness" of the medium which can be, according to him, an advantage for the communication of something complex. He cites several scenarios to illustrate his claim, among them students who try to avoid showing their nervousness by composing a quick, businesslike email to request an extension from their course leader. Others simply want to avoid the discomfort of breaking bad news to people (cf. Thurlow et al. 2004:50). This goes to show that there are also positive sides to supposedly purely disadvantageous features of CMC.

The same holds true for the feeling of anonymity, which has so far been called advantageous for those users who exhibit inappropriate online behavior, as they do not need to fear out-group punishment. In fact, anonymity combined with a certain degree of identifiability can also be beneficial. As Derks et al. (2008:772f.) demonstrate with the help of the success of internet therapy sessions, a certain degree of anonymity of CMC "creates a safer environment for some people to share emotions and to facilitate self-disclosure" (cf. McKenna/Bargh 1999, 2000; McKenna et al. 2002; Neurauter-Kessels 2011). Especially for therapeutic purposes, visual anonymity can have a positive effect on patients' self-disclosure. As Suler (2004: n.p.) points out, "[s] eeing a frown, a shaking head, a sigh, a bored expression, and many other subtle and not so subtle signs of disapproval or indifference can slam the brakes on what people are willing to express". ${ }^{21}$ Virtual presence in contrast to physical presence thus reduces the risk of ridicule or even rejection (McKenna et al. 2002). In these contexts, the communication of emotions is rather reinforced by CMC - especially in the case of displaying feelings in the virtual presence of strangers. In this regard, Internet communication is comparable to the "stranger on the train" phenomenon as described by Derlega/ Chaikin (1977) since

21. It is exactly for this reason that the analyst sits behind the patient in psychoanalysis, in order to remain a "physically ambiguous figure". As the analyst's body language or facial expressions are not revealed to the patient, he does not feel inhibited by the analyst's physical reactions (Suler 2004: n.p.) 
[c]yberspace makes talking with strangers easier. The fundamental point of many cyber-realms, such as chat rooms, is to make new acquaintances. By contrast, in most urban settings, few environments encourage us to walk up to strangers and start chatting. In many cities, doing so would amount to a physical threat.

(Kang 2000: 1161)

Above that, Spears/Lea (1994) point out that co-presence with and identifiability to the in-group can also lead to potential support within an intergroup context, as a sense of solidarity can be created (Douglas/McGarty 2001:401). We must, however, keep in mind that unlike in FtF, self-disclosure in CMC cannot be validated easily due to limited social cues and the absence of nonverbal displays. It can therefore also be used as a deliberate means of deception (see Section 6.2.2).

Statements that uninhibited behavior appears more often in CMC than in $\mathrm{FtF}$ and is furthered by, or worse, is even a characteristic of this form of communication, as purported by early research, are thus far too general to contain any informative value. Personality and social context variables play a more significant role than the features of the communicative platform per se. In conclusion, there is no evidence to assume that $\mathrm{CMC}$ is incapable of expressing and therefore lacking emotions. Although there are no direct and systematic comparisons of conversations treating the same topics in $\mathrm{CMC}$ and $\mathrm{FtF}$ to irrefutably prove this claim, it is nevertheless safe to assume that socio-emotional content is indeed a vital part of CMC (cf. studies of Baym 1995; Werry 1996). The development of personal relationships mushrooming all over CMC, especially with regard to the raging success of purely social services (e.g. Internet dating, support lists, instant messaging, message boards) and the skyrocketing numbers of participants in numerous social network sites (e.g. Facebook, Qzone, Orkud and MySpace), proves this claim to be right. The level of socio-emotional content in CMC is thus greater than once anticipated, which is why "cues-filtered-out approaches display a myopic bias toward face-to-face communication" (Avgerinakou 2003:275). What we need is a "shift of focus from medium-related to user-related patterns of language use [in CMC]" (Androutsopoulos 2006:421). As has become obvious by now, it is less the medium or the form of communication but rather the diversity and creativity of its users that shape (not only) the language of CMC. 



\title{
Communicating via networks II
}

\author{
A social perspective
}

\subsection{Introduction}

The Internet is not about technology, it is not about information, it is about communication - people talking with each other, people exchanging e-mail, people doing the low ASCII dance.

(Strangelove 1994)

As implied by Strangelove, the purpose of the Web is no longer merely the fast transmission of information; it has in fact become an important place for social encounter. The vast majority of people communicating via computers use the Web "in search of social interaction, not just sterile information" and 80\% look for "contact and commonality, companionship and community" (Smolowe 1995:20) - findings which exhibit a great resemblance with motives for FtF interaction. While the Internet and the Web are nothing but the technology behind CMC - Thurlow et al. (2004:29) compare it to the hardware - cyberspace is a synonym for the place of interpersonal interaction as it "is about the people who use the Internet and the different uses they put it to" (ibid.).

Cyberspace can also be considered an amalgamation of social hypertexts, since some of their nodes are becoming representations of people (Erickson 1996: n.p.) and their online relationships. In this way, it virtually brings people together from all over the world for them to discover that they share specific interests or emotional needs. What is interesting is the fact that cyberspace unites people in virtual co-presence who would otherwise never meet in real life, e.g. due to differences in geographical location. As a matter of fact, chances to find like-minded people for special interest topics are by far better online than in one's immediate FtF neighborhood (cf. Schütte 2002:351; Suler 2005). Bonding over these shared topics and related values with virtual peer groups can result in the formation of friendships and other close relationships. As the saying goes "Birds of a feather flock together", users are looking for people who are, or at least appear to be, just like them (see the need for association in Section 4.3.1). Message boards, among other types of CMC, offer the ideal place to do just that. Demonstrating their affiliation, users can gather in smaller groups to swap ideas on their mutual hobbyhorse by engaging in stimulating discussions and interesting 
chit-chat, by exchanging the latest news or also by dealing with important issues of their everyday lives and giving advice to each other. In a nutshell, users can "speak" their minds about (almost) anything with anyone, anytime.

This chapter aims at outlining the most important aspects of social activities online, especially with regard to the message board system The Student Room (TSR), surrounding the following questions: How can we describe the people who populate and thus animate TSR? Should we speak of online groups or communities to describe their forms of gathering? How do these kinds of get-together differ when compared with their offline counterparts? How do codes of conduct emerge online?

\subsection{Gathering online: In groups or communities?}

While meeting and communicating online is an easy thing to do, giving this form of gathering a name, however, is much more difficult. Both notions, groups and communities, are by their very nature hard to define and highly ambiguous. Certainly, factors like member size, degree of interrelatedness, stability (of the common interest topic or purpose of meeting as well as of the participants) and regularity play an important role for choosing one term over the other. But how many people does it exactly take to turn a group into a community then? How much interrelatedness and regularity is necessary for either of these? As usual, the boundaries blur right before our eyes.

While the term group seems to stand for a rather loose and non-binding assemblage of individuals, community entails a different connotation as it has become

a convenient label for a whole range of feelings and ideas about people in tightknit, clearly identified, politically coherent collectives [which] is often used as a rhetorical device for communicating a sense of comforting or reassuring togetherness.

(Thurlow et al. 2004: 109)

Community thus describes the get-together of users who know each other quite well and encounter each other on a regular basis. This is also mirrored in the following quote, the first and partly clumsy definition of online communities as proposed by Rheingold (1993:5):

Online communities are social aggregations that emerge from the net when enough people carry on those public discussions long enough, with sufficient human feeling, to form webs of personal relationships. 
Ever since this first articulation of online community, the notion has become a regular buzzword in CMC research (e.g. Cherny 1999; Werry/Mowbray 2001). Critical voices (e.g. Fernback/Thompson 1995; Jones 1995), however, strongly advise against overextending the term to the point of meaninglessness, as every group seems to be elevated to the rank of a community automatically (Herring 2004). Apart from this pragmatic concern, Herring (referring to McLaughlin et al. 1995) further voices a "philosophical skepticism that virtual community can exist at all, given the fluid membership, reduced social accountability, and lack of shared geographical space that characterize most groups on the Internet" (2004:343). Although this criticism is certainly valid for the first reason given by Herring and acceptable for the second, the last aspect clearly misses the target since "[y]ou don't have to be living next door to someone - or even in the same country - to feel close to them, to share an interest with them or a sense of belonging" (Thurlow et al. 2004: 109). Long-distance relationships certainly are the best counterexample.

It is true, however, that it is hard to get an idea of a certain community with memberships fluctuating as much as in message boards. Investigating one unified community is thus dangerous, as members belong to several, often overlapping communities (Launspach 2000:89). As to the claim of reduced social accountability, we have seen in Section 2.6 that there are indeed mechanisms that help to create a sense of affiliation between the partners by providing some kind of information about the users, not unlike that knowledge we have about each other in a casual FtF group. Even in real life, we never know everything but only random bits and pieces about the background of our fellow students, colleagues or sports partners.

Obviously, the differences between online and FtF groups do not appear as tremendous as they used to but rather seem to melt away. This impression is supported by Benwell/Stokoe (2006:278), who came to the conclusion that "virtual worlds strive to recreate conditions of RL [real life] rather than forge radically new ways of conceiving of relations, communities and identity" (original emphasis). This is also noticeable in the way users disclose information online. According to Yum/Hara (2005), in early stages of typical FtF as well as CMC interactions, nonintimate, impersonal topics are dealt with, while a more intimate level of information is reached over time. The recurring reproach that cyber communities were unreal, entirely populated by consciously constructed pseudo-identities, and thus an alternative rather than an adjunct to real life (cf. Rheingold 1993; Turkle 1995; Parks/Floyd 1996) does not hold: Virtual communities are as real or unreal as any FtF community, which is proven by Anderson (1983:6): "All communities larger than primordial villages of face-to-face contact (and perhaps even these) are imagined". Although the term cyberspace certainly has a science-fictional sound 
to it, interaction in online fora should not be viewed as existing in an independent reality, separate and apart from offline environments, bodies, and concerns (Kendall 1999:60). We should therefore not make the mistake of considering offline interaction as "more real" than online interaction. The distinction between "real" and "virtual" is thus called into question.

What is more, real life relationships are neither replaced, nor reduced in value. Online relationships should rather be considered as a means to support existing conventional offline bonds or to complement them with new online relationships. Herring (2004:338) states that "many online relationships also have an offline component", a claim which is further supported by Baym (1998: 63), who remarks that "[o]nline groups are woven into the fabric of offline life rather than set in opposition to it. The evidence includes the pervasiveness of offline contexts in online interaction and the movement of online relationships offline".

All of this goes to show that we should be wary of using the term community hastily - online or not. Instead, we should recollect the one underlying constant in all types of communities: the "holding-in-common of qualities, properties, identities or ideas" (Wilbur 1997:8) among users, which leads to the (sometimes temporary) formation of a group in the first place. Based on this assumption, Lave/Wenger (1991) propose the concept of community of practice (CoP), also known as discourse community (Swales 1990), terms which seem adequate for the description of those dynamic and emergent networks within message boards, as both imply the absence of physical (and temporal) co-presence. In contrast to other approaches, a CoP does not classify social groupings on the basis of shared abstract characteristics (such as class or gender) or co-presence (such as neighborhood or workplace), but, true to its name, in virtue of shared practice (Eckert 2006: 683).

Originally developed as the basis of a social theory of learning, Eckert (ibid.) describes a CoP as "a collection of people who engage on an ongoing basis in some common endeavor: a bowling team, a book club, a friendship group, a crack house, a nuclear family, a church congregation". Consequently, very unique ways of doing and talking, beliefs, values and power relations emerge in the course of their joint activity around that endeavor (Eckert/McConnell-Ginet 1998:490). The two necessary prerequisites for the working of groups in general, but even more so for CoPs in particular, are shared experiences and history (cf. the storing function of message boards, Section 2.4), which accumulate over time, as well as a commitment to shared understanding by mutual sense making (Eckert 2006: 683). It is thus necessary to reveal the inner workings of the CoP at hand. To this effect, the social aspect of getting together in The Student Room will be outlined in the following. 


\subsection{Taking a look inside: The community of practice of The Student Room}

In order to contour (the emergence of) online CoPs, Baym (1995, 1997, 2000), herself an active participant and scholar of online communities, proposes to have a look at the following four processes:

- forms of expression (e.g. our talking about our communities)

- identity (e.g. our sense of shared group identity)

- relationship (e.g. our connections and interactions with others in the community)

- norms (e.g. the rules and conventions we agree to live by together)

Although the second point can only be fathomed through introspection by individual users, all the other aspects are easily accessible for outside investigators. While the third and fourth aspect will be in the focus of later paragraphs, the first bullet point of Baym's list will be dealt with immediately, thus revisiting the previous discussion: If we want to find out how message board users actually judge their communicative environment, whether they feel part of a group or rather a community, we need to take into consideration their phenomenological (subjective) account. A first explorative keyword search within TSR revealed that none of the users ever referred to themselves as part of a group. Instead, some examples $^{22}$ for the use of the term community could be found within the corpus (see Section 7.2):

(1) Hello all, I am Andre and just become member of thgis community. I really appreciate this TSR lobby and would love to share my views with others.

(andrewilson, thread \#1: post 17)

(2) [...] Anyway, I hope to eventually become a part of this community. Can't wait to talk to some of you.

(lilythrash21, thread \#2: post 1)

(3) No because I have loads of friends who wouldn't even think of using TSR. It's representative of the young internet community maybe, but not all teans.

(D3M!, thread \#9: post 7)

(4) [...] However, I find TSR a much more civilised community. [...]

( maxfire, thread \#9: post 30)

This result is not surprising considering the fact that TSR itself claims to be a community as outspokenly demonstrated on the website's homepage where one

22. Excerpts from the message board corpus are quoted in the original version, thus including all kinds of spelling and formal mistakes. 
is encouraged to "Join the UK's largest student community". This assessment is further substantiated in the section dedicated to the Frequently Asked Questions, the so-called FAQs. Under the category "About The Student Room", users are informed that " $[\mathrm{t}]$ he student Room is the world's largest and fastest growing student community, with over 250,000 members and more than $16,000,000$ posts on our forum". As this introduction proceeds, the user can read repeatedly about the "overall community wellbeing", "the entire user community", "the TSR community" and so on. This insight into the self-awareness of TSR creators as well as users certainly complements our own objective perspective as outside observers. Using the notion of community (of practice) thus seems a justifiable choice.

\subsubsection{Social roles revisited: The hybridization of the private and public}

As we have seen, virtual communities are best defined as "collectivities of people bounded by technology and common interests rather than common location" (Benwell/Stokoe 2006:248). Concentrating on the latter part of this quote, the diverse implications of the lack of "common location" for message board interaction need to be spelled out. Totally disengaged from "normal physical and spatial reference points" (Lea/Spears 1995:202), the only "location" users have in common is indeed the virtual space of the message board. Consequently, several traditional boundaries can no longer be upheld and social spheres that were once distinct overlap (Meyrowitz 1985:5). This includes the distinction between personal and mass communication (Parks/Floyd 1996; Lea/Spears 1995) or the separation of profession and leisure in the sense of the former strict difference between office and home time (Schütte 2000: 148; Avgerinakou 2003:277). Although users interact from their own secluded and secure private places, they send their messages to the relatively open public place of the message board. This obvious blending or hybridization of the private and the public sphere (Schütte 2000: 147f., 2002:353) brings one sphere into the other, thus creating new constellations of social spheres separate and apart from real life contexts. In this "polycontextual situation" (Avgerinakou 2003:282), message board contributions are borne out of "the naturalness, comfort and relaxation which could arguably be expected in a familiar private space" (ibid.).

The result is rather irritating as very personal content and intimate revelations are publicly displayed and distributed on the WWW for anyone to read anytime. This is partly due to the aforementioned fact that users tend to perceive themselves as rather anonymous citizens aloof from a very heterogeneous global village. Since users feel that they cannot be traced back, they sense a freedom to open up even to a completely unknown, physically non-present public (see 
Section 2.5.2). This effect is even strengthened by the knowledge that a simple deregistration can end the membership in such an online community at a moment's notice, which accounts for the general perception of this form of communication as an ephemeral phenomenon, largely devoid of any social obligations (cf. Schütte 2002:346). Paradoxically, the opposite is also true, since considering oneself an integral part of a stable, clearly delimited group of close online friends can actually have the same effect of disclosing behavior and result in feelings of familiarity and comfortableness. This goes to show that the way we interact with each other online and, even more important, how we perceive this interaction bears witness of the changed social prerequisites in message boards. Agreeing with Meyrowitz (1985), these have a strong effect on social behavior: Social stages on which we perform our roles are rearranged and the sense of what is appropriate and what is not can be altered (cf. Avgerinakou 2003:277). It is thus imperative to have a close look at the (emergence of) online codes of conduct in our message board (see Section 3.3.2).

But there are other medium-specific phenomena, which can be traced back to the changed communicative prerequisites: Contributions to message boards are implicitly more often than explicitly "mehrfachadressiert" (Schütte 2000:148), which means that they are addressed to more than one interlocutor within the board at a time. The following examples show how users explicitly address "everyone" in their contributions:

(5) hi all, i'm frankie :) introduce yourselves to me! :) hey, i'm Frankie, i'm 17 and from Nottinghamshire, UK. i'm currently awaiting my AS results which is in 8 days, oh dearr! (9) panicpanic!!!! 101898989

aaanyway haha.. i love fine art, expressionism, oil paint (just getting covered in paint really lol) and i like Drama too, and Law. :) i enjoy shopping and long walks on sunny days $:$ i i ADORE chocolate, alottt!

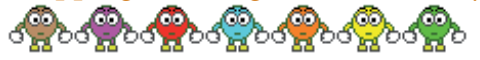

(superfrankie, thread \#1: post 1)

(6) Your pearls of wisdom to university freshers...

Hello everyone.

I'm in abit of a dilemma, i need to write an article about student lifestyle but have no idea where to begin.. and as im a fresher myself... i wouldnt have a clue $\odot[\ldots]$

(Sahds, thread \#42: post 1)

(7) Best universities for english?

Any ideas anyone. are there any official league tables etc out there. so far i'm considering oxford and durham.....

(Selenax, thread \#45: post 1) 
Typically, thread starters explicitly address their audience one way or another, by means of the heading or the entry itself, as part of a question or through a greeting. But who exactly is "everyone"? Goffman's (1981) reflections on production formats on the one hand and on the participation framework on the other help us not only to understand the importance of multi-addressing but also to point out the differences between $\mathrm{CMC}$ and FtF interaction. On the receiving end of communication, Goffman distinguishes between ratified and unratified recipients. Among the former, there are those directly addressed by the user's post and those who are not directly addressed but who are nevertheless ratified to follow the ongoing conversation, such as regular group members other than the actual interlocutor. At this point, the differentiation between addressed and unaddressed recipients blurs, however, for the following reason: In contrast to FtF conversations, users usually do not converse with an individual (especially not by keeping eye contact with him) but with the vaguely delineated, disperse audience within the entire $\mathrm{CoP}$ of the particular thread. Although this audience is seldom verbally addressed in terms of greetings or other terms of address (Hi group!, Hello all!, Hey guys! etc.) as in the above examples, it is nevertheless addressed implicitly or at least cognitively by users composing their contributions. It is thus questionable whether there are indeed unaddressed recipients among those who are ratified to "listen". Multi-addressing - at least as a cognitive construct in the users' minds rather than a verbal condensate in users' posts - thus becomes an indispensable, medium-specific necessity.

In treating Goffman's second, unratified type of recipients, another slight medium-specific modification is called for. Again in contrast to FtF, we can and should definitely expect a lot of invisible and unratified recipients in message boards, who are termed lurkers in CMC environments. We can find overhearing lurkers, who inadvertently or haphazardly "stumble" across message board entries, and eavesdropping lurkers, who take interest in ongoing discussions for the sake of scientific or commercial purposes. Similar to FtF interactions, these roles are not set in stone, as uninvolved and unaddressed lurkers can easily turn into ratified, addressed recipients by actively joining the ongoing conversation. In my view and thus deviating from Goffman's original classification, lurkers are the ones to earn the label unaddressed recipients, which leads to the following modified categorization (see Table 1).

Table 1. Types of message board recipients (Goffman 1981, modified)

\begin{tabular}{ll}
\hline Ratified recipients & Unratified recipients \\
\hline - addressed individual users & - unaddressed overhearing lurkers \\
- addressed users in the CoP & - unaddressed eavesdropping lurkers \\
\hline
\end{tabular}


The other side of the communication process is interesting to look at as well. The contributing message board user is principal (the one who triggers the process of text production), author (the one who writes the contribution) and animator (the one who delivers the contribution to the audience - in our case at least by clicking on a "send" button) all in one. This is atypical for mass media where all these productive steps and roles are normally realized by different people at different times. While the producing side of the model still resembles very much private $\mathrm{FtF}$ communication, the receiving side is already well adopted to the conditions of mass communication - a clear-cut case of hybridization.

But there are other social roles besides that of producer and recipient that can be found especially in CMC environments such as message boards, which can no longer be defined with recourse to spatial or hierarchical dimensions of FtF interaction. As Suler (2004: n.p.) puts it,

[i]f people can't see you or your surroundings, they don't know if you are the president of a major corporation sitting in your expensive office, or some 'ordinary' person lounging around at home in front of the computer. Even if people do know something about your offline status and power, that elevated position may have little bearing on your online presence and influence.

(Suler 2004:n.p.)

Accordingly, users are predominantly judged by their online appearance and therefore have an equal opportunity to voice themselves since they "start off on a level playing field" (ibid.) regardless of their real life status, wealth, race, gender, etc. We should not forget, however, that new internal power hierarchies do develop, which is why utopian claims that CMC is inherently egalitarian are compromised again (cf. Herring 2001:624). Among these new hierarchies is the broad classification of users into experienced insiders or regulars and so-called newbies. As we have seen in Section 2.6, there are several indicators for a user's status in TSR boards (and most other boards for that matter): Labels (for example "New Member", "Exalted Member"), the joining date, the number of posts and the reputation system all help to tell apart "regulars" from "newbies". While regulars are familiar with the underlying conditions of communicating ideas via message boards, newbies are rather inexperienced newcomers, who still have to learn how to communicate, bond and behave online. First of all, they are supposed to study the FAQs and observe online conversations passively. Later, by contributing regularly and constructively and by behaving according to the rules, newbies can change their status over time and thus become full-fledged message board regulars. Although it is hard to tell where to draw the line between a core member and a sporadic contributor, this classification is nevertheless vital as it reveals the only concrete clue to the underlying power structure within a board. Further insights into prevalent social bonds among contributors can be gained by looking at the 
buddy lists or by comparing users' memberships in "societies" (see 2.6.2) within the board to detect commonalities.

The last differentiation in terms of roles to be pointed out here is the one between moderators and all the other members. Those responsible in TSR, the (paid) administrators (short admins), give established and knowledgeable members the opportunity to assume responsibility and oversee specific boards. In becoming TSR moderators (of varying hierarchical status such as supermoderators, global moderators and minimods, further also forum assistants), users are supposed to set a good example for appropriate online behavior as a by-product of their ongoing conversations. While this actually holds true for each and every member of the board, moderators have additional rights and powers in that they can edit, delete or censor inappropriate posts (by replacing swear words with a row of asterisks such as ${ }^{* * 23}$ ) and caution their authors (by assigning red gems, viz. "repping" them, see 2.6.2). They can also close entire threads and restrict, suspend or even terminate memberships - even without previous warnings. Although monetary fines or even physical violence are not at their disposal when it comes to sanctioning misguided users, moderators still have considerable influence on the establishment and execution of the code of conduct, which is why it is certainly helpful to spot those moderators and keep an eye on their behavior and its possible influence on others. "Normal" users, on the other hand, also have a variety of informal sanctions at their command, as they can insult, parody or inform the "bad seed" about his misbehavior; the most effective measure is certainly giving him the silent treatment (Kollock/Smith 1996:124). What actually needs to be sanctioned is stipulated in the code of conduct of TSR. As a helpful compass for appropriate online behavior (obviously not only for newbies), TSR netiquette will be dealt with in theory and in practice in the following.

\subsubsection{The emergence of norms and codes of conduct}

A common code of conduct, or "norms of practice" (Herring 2001:622), is yet another indicator for the fact that we are indeed dealing with a virtual community of practice. In all communities, online or offline, "rules of conduct tend to be organized into codes which guarantee that everyone acts appropriately and receives his due" (Goffman 1967:55). It is not surprising that different compositions of CoPs as well as their users' diverging intersubjective expectations result in

23. Although the FAQs clearly advise against this procedure, users nevertheless use asterisks themselves to veil (part of) their swearing. For this reason, it is hard to tell whether a post has been censored by the moderators or whether asterisks were already part of the original version of the entry. 
varying and very group-specific codes of conduct. Although we can make assertions about the emergence and the overall contents and functions of such rules in general, we still have to consider the TSR guidelines in particular.

Since Foucault (1980), we know that social institutions are constructed and maintained through discourse alone - an insight which holds especially true for online assemblages as in message boards, where social structures are created exclusively out of words (Rheingold 1993; Kollock/Smith 1999). This type of discourse generates rules, sanctions against the violation of these rules and consequently also a system of governance as described above (see moderators) to enforce these sanctions (Herring 2001:624). But how do online norms come into being in the first place? In accordance with Grice's maxims, the need for co-operation and even more so for mutual respect motivates users to cast their "rules of the game" into a relatively solid form, known as the netiquette, a blend of the two nouns net and etiquette. Etiquette, meaning "the forms required by good breeding or prescribed by authority to be required in social or official life" (Shea 1994: 19), is etymologically rooted in the French word for ticket: "If you know the etiquette for a particular group or society, you have a ticket for entry into it" (ibid.). The netiquette as a collection of rules and guidelines draws users' attention to what is considered as polite, respectful and sociable behavior online. This includes norms aimed at

preventing others from having to read useless material, limiting the extent to which one can fictionalize identity, protecting other users' privacy, retaining attribution when following up on ideas, and remaining readable.

(Baym 1995: 159)

This may even include instructions for the appropriate amount of emoticons. The core rules of netiquette, standardized catchphrases such as "Remember the human", "Lurk before you leap" and "Respect other people's time and bandwidth", to name but three, can be encountered all over the web. ${ }^{25}$ These basic rules are generally agreed upon and thus apply throughout almost all of cyberspace, as they are direct reflections of a pre-existing social and cultural consensus among social actors and thus common (FtF) courtesy (Goffman 1955).

24. Bandwidth, as defined by Shea (1994:39), is the capacity of the wires and channels to carry information or of host systems to store this information. In the past, this capacity was highly limited. A note posted five times to the same thread or one that is longer than necessary did waste bandwidth back then as it still wastes users' valuable time today (cf. Kollock/Smith 1996).

25. A comprehensive list of rules of netiquette can be found in Shea (1994). 
TSR netiquette forms part of the so-called FAQs (Frequently Asked Questions). They identify the purpose of the community and address those recurring issues which are concerned with the overall handling and functions of the communicative platform as such. Consequently, this is also mirrored in the netiquette, which does not only detail common FtF norms to spare the feelings of our interlocutors, but also spells out rather medium-specific codes of conduct concerning what is appropriate online behavior. Accordingly, these norms are always "infused" (Kiesler et al. 1984:1126) by those who developed and used electronic communication for a relatively long time: A distinctive subculture of computing professionals and "die-hard" regulars. As these are the individuals with the knowledge of the dayto-day workings of the CoP and its challenges, they are given the opportunity to modify the prevalent rules, which should consequently stand better chances to fit to local conditions (Kollock/Smith 1996: 121). Another advantage of this joint venture of shaping the terms and conditions of getting together lies in the fact that those rules were not forced upon members by some unknown, outside authority but emerged from their midst. Note, however, that this does not mean that every single member has a say about these rules. In fact, only those major in rank can be considered responsible for their generation. It is, therefore, by no means certain whether everyone even reads those rules before posting, let alone whether users actually attach value to those guidelines and conduct themselves accordingly.

As mentioned before, rules are not only made by those in charge at TSR, but are also monitored by them. The sword of Damocles, in terms of the moderators' sanctions, thus always hangs over the users' heads to prevent the exploitation of loopholes in the following rules (URL 9):

1. No personal attacks or inflammatory behaviour.

2. Post in the correct forum and stay on topic.

3. No cheating.

4. No advertising or inappropriate links.

5. Post in the English language unless in an area where it is acceptable to do otherwise (a language society thread or the languages academic help forum). Outside these areas always provide a translation. No text speak.

6. Respect the privacy of other users.

7. Do not condone or encourage illegal activity.

The last two items on this list refer to the protection of users, be it from any kind of illegal activity or from being more exposed than desired (i.e. by posting someone's private pictures or email address etc.). The same holds true for rule number 4, which bans any sort of advertisement or otherwise inappropriate content 
(even if only embedded, for example, via links). The fifth rule also reminds users to be civil and not to swear (neither in words, nor in images, links or attachment), to basically stick to the English language and to steer clear from "text speak" (i.e. CMC specific acronyms and abbreviations, see Section 2.6.2). The third rule pays tribute to the special nature of TSR: It prohibits users' attempts "to ask for information about an exam [they] haven't taken yet or provide information about such an exam" (URL 9). The second advice is very typical for message boards in general, as it asks to uphold the default topical hierarchy. Contributions, always to be provided with a meaningful heading, should therefore only be posted once and in the relevant thread. The second half of the above catchphrase ("stay on topic") is concerned with so-called thread drifts and thread hijacking. In contrast to FtF and despite the fact those in charge recognize that discussions often "diverge and evolve" (ibid.), topics are not supposed to drift, which is why "threads or responses that are not relevant to the initial post will most likely be deleted or split into two different threads" (ibid.).

The first item on that list surely is the most important one for the present study and thus deserves special attention. The "powers that be" specify this rule by defining personal attacks and inflammatory behavior as those actions which are supposed to provoke a negative response from another user. This is sometimes known as trolling or flaming (see Section 6.2.2 and 5.7 respectively), which is mostly based on racist, sexist, homophobic, bigoted, pornographic, or otherwise offensive material. As they put it (URL 9),

[w] expect that you will show respect and sensitivity to the beliefs and views of all users. Comments we believe to be rude, excessively profane, deliberately antagonising or disruptive, or a personal attack will lead to a warning and may result in the involved member(s) being suspended or banned immediately.

Once more, possible sanctions are recalled to mind. More importantly, expectations concerning appropriate users' behavior in dealing with other users and their opinions are spelt out, as they are asked to "show respect and sensitivity" and not to be rude, profane or antagonizing. While all the other rules primarily focused on the handling of pre-set templates in the virtual environment of TSR so as not to irritate interlocutors and endanger the cooperative online behavior, this rule highlights individual's verbal behavior towards his fellow-users. We can thus conclude that discourse, either in terms of saying something or doing things with words, can have effects on interlocutors' needs or wants, which is why both areas are described in the netiquette. 


\subsection{Summary: Why we need to draw on FtF interaction}

"When in Rome, do as the Romans do" - this is what we are told to do in order to fit in when we find ourselves among a new group of people. In our case, Rome is TSR, which is why we need to observe the "TSRians" and their behavior as a community carefully to avoid premature judgments about their social intercourse. To this end, this chapter has been dedicated to giving a first insight into the inner workings of TSR on an interpersonal plane. Since most of the factors known in FtF cannot be taken into account, with shared space leading the way, the only springboard for the promotion of commonality are indeed shared (momentary) interests. For this reason, TSR has been labeled a community of practice $(\mathrm{CoP})$, a term which also alludes to and thereby does justice to its emergent nature. Although this chapter has brought to light more similarities with FtF interaction than expected, some differences still cannot be denied. This becomes obvious when investigating social roles (for example producer vs. addressee, regulars vs. newbies, moderators vs. regular users etc.), which are a direct condensate of the altered communicative prerequisites online. Still, cyberspace mostly aims at recreating real life, which can also be seen in the attempt to cultivate a tailored code of conduct by adopting guidelines from FtF interaction and adapting them for the purposes of the online community. They are not only assembled but also controlled in a cooperative effort. As is often the case, "[s]ocialization comes about by actively participating in the everyday, contingent recreating of accountable action, not by passively internalizing widely shared patterns for ritual behavior" (Arundale 2006:198). Thus, we need to keep in mind that these codes of conduct can only be of approximate value: What is felt to be appropriate always lies in the eyes of the beholder (see the discussion in Chapter 5). In fact, the emergent nature of online communities itself inhibits the consolidation of accurate guidelines that are valid "once and for all". This is why FAQs should be regarded as an adequate starting point rather than the end of the journey, open to constant revision (Graham 2008: 302).

Leaving aside the true nature and development of FAQs and netiquette for a moment, we need to be aware of the fact that it is not at all verifiable whether users do indeed bother reading the netiquette as is recommended. Consequently, we need to assume that users automatically fall back on what they know from real life and what has proven to be efficient there one way or another. The influence of real life on online behavior, at least in terms of evaluations and expectations of what is appropriate and what is not, cannot be underestimated and needs to be investigated meticulously. We will therefore shift the focus, away from the medial setting and its implications for the social sphere, to the human beings behind their keyboards. 
In order to find out about interpersonal online relations, we will have to go back to the roots of FtF interaction and identify the basic human needs and strategies for interpersonal behavior in real life contexts. The next two chapters are therefore dedicated to a number of influential theoretic approaches that deal with the foundations of interpersonal FtF relations. In this context, notions such as mutual respect, cooperation, common courtesy, politeness but also rudeness, personal attacks, trolling, flaming and inflammatory, sociable, appropriate or inappropriate behavior, which have already been brought up throughout this chapter, will be clarified. 



\title{
Interpersonal relations I
}

\author{
The origins of politeness, face \& facework
}

\subsection{Introduction}

[Politeness] is a social lubricant less nocuous than alcohol, probably useful, like free alcohol, for the corps diplomatique [or] a velvet glove within which to hide one or another kind of iron fist [...]

(Sell $\left.{ }^{2} 2005: 112\right)$

On the way to describing interpersonal relations in FtF interaction, we cannot help but stumble across the ubiquitous concept of politeness and its supposedly clear-cut counterparts impoliteness and rudeness at every other step of the way. In dealing with this notional triad step by step, I will take a close look at the concept of politeness - and the neighboring concepts face and facework - by briefly reviewing and discussing some of the most well-known approaches.

From an etymological point of view, the English lexeme polite can be traced back to the Latin past participle form politus, literally meaning "polished", metaphorically, however, also "refined" and "well-mannered" (cf. Stowasser et al. 2003:388). While the origins of this lexeme appear rather unproblematic and clear-cut, the understanding of polite behavior could not differ more:

Some people feel that polite behavior is equivalent to socially 'correct' or appropriate behavior; others considered it to be the hallmark of the cultivated man or woman. Some might characterize a polite person as always being considerate towards other people; others might suggest that a polite person is self-effacing. There are even people who classify polite behavior negatively, characterizing it with terms such as 'standoffish', 'haughty', 'insincere', etc.

(Watts 2003:1)

As this quote shows, politeness varies extremely with regard to the meanings and connotations individual speakers associate with it, resulting in a bewildering ambiguity in the use of this term. Members of speech communities possess clear beliefs about and are capable of immediate and intuitive assessments of what constitutes polite or rude, tactful or offensive behavior in ordinary, daily contexts of use (Pizziconi 2006:679). Although speakers agree on the existence and share knowledge about the rules of politeness, i.e. of norms and principles to ensure a smooth flow of conversation, linguists have difficulty putting their fingers on its 
texture. Obviously, we are dealing with a case of "I know it when I see it". In fact, it even seems easier for us to grasp politeness ex negativo, as behavior which is in line with the current set of rules usually does not attract any attention - contrary to the lack thereof, which is absolutely noticeable (Haferland/Paul 1996:29).

The definitional problem arises because politeness as a purely mental notion is strongly dependent on the interpreting mind in terms of scope of applicability, i.e. a person's willingness to label an utterance or an action polite. More often than not, politeness depends on the evaluation of individual interlocutors at individual moments in individual circumstances. Accordingly, politeness is also a highly context-sensitive phenomenon. To make matters worse, it is extremely flexible with regard to its superficial manifestation on the utterance level, which is why there is no such thing as a polite utterance per se but only utterances with a certain potential to be perceived by interlocutors as polite or not. This explains why the search for a standardized definition applicable to all sorts of contexts is bound to fail right from the start.

Following Eelen (2001) and Watts (2003), no less than nine different, sometimes even contradictory approaches set out to tackle this definitional problem and find a basic point of departure for a phenomenon which can be considered as one of the marshiest fields within pragmatics. Nevertheless, it has been the focus of scientific research across disciplines, especially pragmatics, since the late sixties of the last century and has not lost any of its appeal for the academia to this very day. ${ }^{26}$ In line with previous research, this chapter also sets out to disentangle the complex anatomy of the concept of politeness. Some classic approaches which have been poked and prodded, criticized, revised and extended in empirical and theoretical studies of various disciplines to this very day are sketched in broad strokes. Special focus will be put on the introduction of Brown/Levinson's (1978) seminal work, which will be supplemented by its predecessor, Erving Goffman's (1967) sociological approach.

26. This can easily be proven simply by looking at the stunning amount of literature documenting the interest in the study of interpersonal relations. In 1999, Fraser observed that there are over a 1000 books, papers and articles published on the concept of politeness, among them influential research monographs and collections such as Eelen (2001), Mills (2003), Watts (2003), Locher (2004), Lakoff/Ide (2005) and Locher/Bousfield (2010), to name but a few. An incredible amount of topic related papers can also be found in the Journal of Pragmatics and in The Journal of Politeness Research, which was launched in 2005 to fill the need for a periodical publication format dedicated to politeness research alone. 
Table 2. Lines of investigating politeness

Prepragmatic approaches

- The social norm view
Pragmatic approaches

- The conversational maxim view

- The face-saving view

\subsection{Fraser, Lakoff and Leech: Some classic views on politeness}

The astonishing number of approaches which are concerned with the demystification of the nature of politeness can be summarized with the help of the following skeletal overview. While the distinction between prepragmatic and pragmatic approaches ${ }^{27}$ is based on Held $\left(1995,{ }^{2} 2005\right)$, the naming of the lines of investigation within this dyadic distinction is directly borrowed from proposals by Fraser (1990:219) and Thomas (1995:158) (see Table 2).

\section{The social norm view}

Even before the boom of pragmatic engagement in the study of politeness in the 1970s, Romance and German linguistics had already taken an interest in this field and assumed that politeness strongly depends on the underlying culture-bound ideologies and norms. Behavior that conforms with these fixed sets of rules would be considered polite, while actions that were not in congruence with the norm would be evaluated as impolite or even rude behavior (Fraser 1990: 220). However, the logical consequence of this approach, according to Held, is the incapacitation of the individual speaker insofar as they are not acting intentionally anymore but are turned into a "creative representative of a language community (1992:138f.) instead. The question we thus have to answer in this context is whether language (including manifestations of politeness) is a purely social product based on the norms and values of the respective community that lie beyond the control of the rational individual or whether it is, in fact, the outcome of the unique and creative cognitive system of the individual speaker. As is often the case, we cannot think in terms of a black-and-white solution but have to adopt a middle position. As speakers are at least generally aware that such norms and principles exist in the society at large and in particular situational encounters, to be polite would thus mean "to adapt yourself to different situations [and to behave] according to the expectations of the place" (Blum-Kulka 22005:259).

27. A similar proposal for the structuring of the field is put forward by Werkhofer $\left({ }^{2} 2005: 156\right)$, the only difference being his rather unspecific and hence unfortunate choice of terms of traditional and modern approaches. Nevertheless, they give us a clue as to where to place the socalled postmodern approaches which will be introduced in Chapter 5. 
Closely related to the social norm view is Fraser's sociolinguistic view of politeness (1990), the conversational contract view. Although treated as a completely distinct approach to politeness, Fraser nevertheless seems to describe the logical consequence of the social norm view. He considers politeness to be the default setting in conversational encounters in which participants simply fulfill what Fraser calls the conversational contract (CC):

I assume that whenever two individuals [...] engage in serious conversation they establish a conversational contract. On entering into a given conversation, each party brings an understanding of some initial set of rights and obligations visà-vis the other. These may be a conventional set, for example, when two people meet for the first time on the street, or this set may be determined by previous conversation and knowledge of the person they are talking with. During the course of conversation, there is always the possibility for an ongoing renegotiation of this conversational contract, an ongoing readjustment of just what rights and what obligations each has towards the other.

(1980:343)

Accordingly, an utterance would be considered polite if the (rational) speaker abides by the rules of the relationship and does not violate the rights and obligations prevailing in that specific encounter, at least not in the eyes of the hearer (Fraser 1980:343f.). Politeness is thus expected to exist in every conversation even though participants do not necessarily recognize someone as being polite - after all, this behavior is the norm. As stated before, only deviations from this norm attract attention. What is also stressed in the above quote is the fact that politeness is a collaborative endeavor undertaken by rational participants who pursue a common (communicative) goal for the time of the actual exchange. Therefore, politeness is an extremely instable element and subject to change.

In contrast to Fraser's (1990:221) conclusion, which holds that the social norm view has few adherents among current researchers, Bousfield (2008:45) claims that "a divorce from the social norm approach for linguistic conceptualizations of $\mathrm{im} /$ politeness may not only be a mistaken move, but, theoretically, an impossible one". It would thus not only be imprudent but also impossible for a sensible analysis of politeness to completely factor out cultural and group norms of the speaker's social background. After all, a weak reading of the social norm view in combination with other approaches obviously cannot be condemned that easily.

\section{The Conversational maxim view}

In contrast to the aforementioned prepragmatic view, the pragmatic approach to politeness abandons the rigid straightjacket of social norms, shifting the focus to the rational individual and the "intentional, goal-oriented, situation-specific selection of linguistic strategies between ego and alter" (Held ${ }^{2} 2005$ : 133). In this quote, Held addresses several important issues which researchers tried to include 
little by little into their considerations when setting out to design a model that would do the phenomenon of politeness justice.

The conversational maxim view is usually equaled with Lakoff's Conversational-maxims approach (1973) and Leech's Politeness Principle (1983). Both of their propositions rely on Grice's seminal paper "Logic and conversation" (1975) which not only introduced the Cooperative Principle $(C P)$ and its maxims of quality, quantity, relation and manner, but also inspired research in politeness and sparked off the entire pragmatic turn. In her paper "The logic of politeness, or minding your p's and q's" (1973), Robin T. Lakoff tries to find reasons for those cases in which maxims are not observed and locates them in the field of politeness. Unfortunately, though, she never defines her understanding of politeness. ${ }^{28}$ Lakoff (1973:297) articulates her view of politeness in the form of three pragmatic rules, which have attracted a lot of criticism for their scant formulation and their vague significance:

1. Don't impose;

2. Give options;

3. Make A feel good, be friendly.

As Fraser (1990:224) notices, these rules can be at times reinforcing each other, at other times be in conflict with each other. Although formulated as imperatives, this tripartite set of rules shall not be mistaken for a production model of politeness. Lakoff places her "rules of politeness" side by side with Grice's $\mathrm{CP}$, which she renames "rules of conversation". While Grice's maxims are subsumed under the one, overarching maxim "Be clear", Lakoff entitles her set of maxims "Be polite", with both sets of maxims constituting "Pragmatic Competence" (see Table 3).

Lakoff concludes this comparison with the assessment that, most of the time, politeness takes precedence over clarity in cases where the two conflict. After

Table 3. Lakoff's two-sided model of pragmatic competence (Watts 2003: 60, adapted)

Pragmatic competence

Rules of conversation

Be clear. (= Grice’s CP)

R1: Quantity

R2: Quality

R3: Relevance

R4: Manner

\section{Rules of politeness}

Be polite.

R1: Don't impose.

R2: Give options.

R3: Make A feel good - be friendly.

28. In her later works, however, Lakoff is more explicit and refers to politeness as "a device used in order to reduce friction in personal interaction" (1979:64). 
all, the avoidance of offense is considered more important than the achievement of clarity, "since in most conversations, actual communication of important ideas is secondary to merely reaffirming and strengthening relationships" (Lakoff 1973:297f.). Abiding by the rules of politeness, thus inevitably leads to the breaching of the rules of conversation, which is ultimately the reason why the $\mathrm{CP}$ is violated fairly regularly.

Similar to Lakoff's proposal, Geoffrey Leech, in his monograph Principles of Pragmatics (1983), also leaves the CP unaltered and adds a politeness principle (PP), which regulates the "social equilibrium and the friendly relations which enable us to assume that our interlocutors are being cooperative in the first place" (1983: 82). Leech elaborates this claim with the help on his six maxims: tact, generosity, approbation, modesty, agreement, and sympathy. Following Lakoff, he places his PP on the same level as Grice's CP and considers it "a necessary complement, which rescues the CP from serious trouble" (1983:80) as it gives reasons why speakers deviate from the Gricean maxims as often as they do. He illustrates this claim with the following classic example (1983: 80):

A: We'll all miss Bill and Agatha, won't we?

B: Well, we'll all miss BILL.

In this short piece of dialogue, B obviously flouts Grice's maxim of quantity, certainly for a good reason, $v i z$. he did not want to sound offensive by giving the full information, which is nevertheless implicated here ("we won't miss Agatha"). As Watts argues, the "discourse marker well [...] indicates a constraint on [B's] ability to uphold the CP by abiding to the PP" (2003: 65). The two principles seem to be in conflict here and the speaker has to decide for himself which principle to follow.

\subsection{The face-saving view: Brown/Levinson's Politeness Theory}

It seems, however, that no matter where one arrives with politeness, one must begin with Brown and Levinson.

(Meier 2004:7)

Probably the most widely received approach to politeness to this very day was proposed by Penelope Brown and Stephen Levinson in their 1978 essay "Universals in language usage: Politeness phenomena”, once again published in 1987 as a monograph, Politeness. Some universals in language usage. True to Meier's introductory quote, no study concerned with interpersonal relations can do without key concepts of Brown/Levinson's seminal work, which therefore earns the label Politeness Theory or, owed to the central notion of face, the face-saving view (Fraser 1990). 
Brown/Levinson also support Grice's view and adopt the assumption that interlocutors' (verbal) behavior is by its nature rational and efficient. In accordance with Lakoff and Leech, they also justify the temporary non-acceptance of Grice's maxims with considerations for politeness, among - as they concede - other possible motivating forces such as irony, sarcasm or humor. The focus of their model rests, however, exclusively on politeness.

\subsubsection{Key concepts and inner workings}

Brown/Levinson present a speaker-centric model in which the slot of an actual speaker is filled with a cardboard figure, since all assumptions are based on a model person (MP) with the "ability to rationalize from communicative goals to the optimal means of achieving these goals" (Watts 2003: 85). The hearer, however, only serves as a parameter for the MP's assessment of the most appropriate politeness strategy in the current situation. How he might actually react to the chosen strategy is never an issue in Brown/Levinson's paper. The conception of both the speaker and the hearer in this model has earned them serious critique. Following the aforementioned theories of Lakoff and Leech, the fundamental goal of politeness for Brown/Levinson is also the maintenance of social cohesion. The key to achieving just that, however, lies in the well-known basic notions of face and face wants.

Brown/Levinson's rational model person is assumed to have face, an individual's "public self-image that every member [of a society] wants to claim for himself" (1987:61). Although both authors acknowledge that face is a culturesensitive notion whose content will be subject to "cultural elaboration" (1987: 13), they still assume that "the mutual knowledge of members' public self-image or face, and the social necessity to orient oneself to it in interaction, are universal" (Brown/Levinson 1987: 61f.). Despite this claim to universality, the picture Brown/ Levinson have drawn of face still exhibits a Western ethnocentric orientation. As to the nature of face, they add the following reflections:

Thus face is something that is emotionally invested, and that can be lost, maintained, or enhanced, and must be constantly attended to in interaction. In general, people cooperate (and assume each other's cooperation) in maintaining face in interaction, such cooperation being based on the mutual vulnerability of face. That is, normally everyone's face depends on everyone else's being maintained, and since people can be expected to defend their faces if threatened, and in defending their own to threaten others' faces, it is in general in every participant's best interest to maintain each other's face [...].

(Brown/Levinson 1987:61) 
Based on this definition of face, Brown/Levinson distinguish two separate types of face. ${ }^{29}$ Note, however, that the choice of terms is rather unfortunate since positive and negative are not meant to be evaluative terms for 'good' and 'bad' as one's intuition might suggest but as two opposite poles on a scale:

negative face: "the want of every 'competent adult member' that his action be unimpeded by others" (1987:62) or "his want to have his freedom of action unhindered and his attention unimpeded" (1987:129).

positive face: "the want of every member that his wants be desirable to at least some others" (1987:62) or "his perennial desire that his wants (or the actions/acquisitions/values resulting from them) should be thought of as desirable" (1987:101).

A speaker's face is closely linked to so-called face wants, "which every member knows every other member desires, and which in general it is in the interests of every member to partially satisfy" (Brown/Levinson 1987:62). Roughly translated, one could say that a person's negative face is the need to be independent, to have freedom of action, and not to be imposed on by others. The positive face, on the contrary, focuses on the need to be accepted, even liked in a group and to be treated that way. They mirror what O'Driscoll calls the "duality between association and dissociation" (1996:4), ${ }^{30}$ i.e. the need to "come together, make contact and identify with others, to have ties, to belong, to merge [on the one hand, and] the need to go off alone, avoid contact and be deindividuated, to be independent, to separate" on the other. Tannen elucidates:

We need to get close to each other to have a sense of community, to feel we're not alone in the world. But we need to keep our distance from each other to preserve our independence, so others don't impose on or engulf us. This duality reflects the human condition. We are individual and social creatures. We need other people to survive, but we want to survive as individuals.

(1992: 15)

Having introduced the notion of face we are now in a position to comprehend Brown/Levinson's view of politeness. It is not only a means of showing concern for the other, as was rightly assumed by Lakoff and Leech before, but more precisely a means of showing concern for the other person's face. For that reason,

29. In formulating their bipartite notion of face, Brown/Levinson had in mind Durkheim's (1915) positive and negative rites (Brown/Levinson 1987:43; cf. O’Driscoll 1996:2; BargielaChiappini 2003: 1460).

30. Schopenhauer's (1851) often-quoted example of porcupines trying to get through a cold winter can also be used to explain this duality. Tannen (1992: 14f.) elucidates that "they huddled together for warmth, but their sharp quills prick each other, so they pull away. But then they get cold. They have to keep adjusting their closeness and distance to keep from freezing and from getting pricked by their fellow porcupines." 


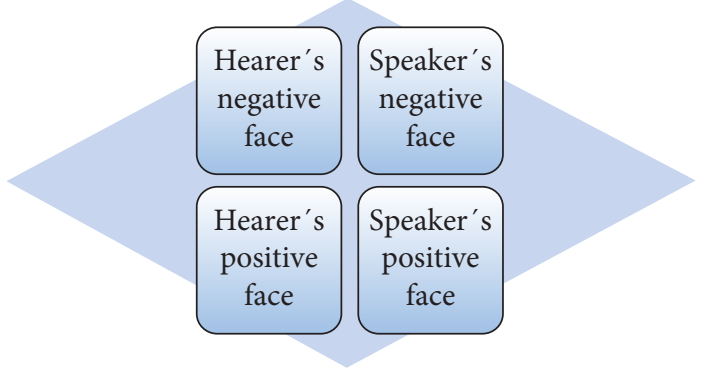

Figure 10. Four directions of FTAs according to Brown/Levinson

Brown/Levinson focus on the maintenance of the other's face and examine very carefully what happens when our interlocutor's face is in fact endangered and what can be done to mitigate that threat. In this context, Brown/Levinson introduce the by now famous notion of face threatening acts (FTAs) with which they associate "those acts that by their nature run contrary to the face wants of the addressee and/or of the speaker" (1987:65), indicating that "some acts are intrinsically threatening to face" (1987:65). This claim, however, has been refuted before, since there can never be a 1:1 relationship between a surface structure of whatsoever form or content and its evaluation in the interpreting mind.

Bearing this strong point of criticism in mind, we nevertheless distinguish four cases of FTAs: Those addressed to the hearer and/or the speaker and those related to the positive or the negative face. Schematically, the possibilities for the direction of FTAs can be depicted in a four-way grid (see Figure 10). Each of these four possibilities is fathomed by Brown/Levinson (1987:65ff.) in the form of detailed and extensive lists. Although this classification seems rather clear-cut, the categories are not mutually exclusive. In fact, there can be overlaps as some acts can simultaneously pose a threat to the negative and the positive face of the hearer and the speaker. According to Brown/Levinson (1987:67), these kinds of crossclassification are evident in complaints, interruptions, threats, strong expressions of emotion but also in requests for personal information.

In order to weaken FTAs, this speaker-oriented production model outlines five strategies for the mitigation of FTAs from which corresponding substrategies are derived. Their five basic superstrategies are summarized in an often cited table (see Table 4). In choosing the appropriate strategy, ${ }^{31}$ speakers will also have to consider

31. The choice of strategies is meticulously illustrated by Yule (1996:62ff.) in his famous example on how to ask someone for a pen. 
the relative weightings of (at least) three wants: (a) the want to communicate the content of the FTA $x$, (b) the want to be efficient or urgent, and (c) the want to maintain H's face to any degree. Unless (b) [and also (a)] is greater than (c), S will want to minimize the threat of his FTA.

(Brown/Levinson 1987:68)

These strategies range from type number one (still a very face-threatening strategy) to type number five, "Don't do the FTA", which stands for the best possible way to protect the other's face by simply avoiding the FTA altogether. The second best way of mitigating face damage is by picking strategy number four, "off record". The speaker avoids unequivocal impositions by veiling their intentions which, of course, necessitates a more complicated inference process on the part of the hearer. Linguistically, this strategy can be implemented by "metaphors, irony, rhetorical questions, understatement, tautologies, all kinds of hints as to what a speaker wants or means to communicate, without doing so directly, so that the meaning is to some degree negotiable" (Brown/Levinson 1987:69). This strategy offers a great advantage to the speaker insofar as they can always make use of its inherent loophole, addressed by Brown/Levinson in the last part of the quote above: Since the speaker's true and maybe face-threatening intentions cannot be pinpointed, they can be denied at all times. As a payoff of this strategy, the speaker can, for example, "get credit for being tactful, non-coercive; [and] he can avoid responsibility for the potentially face-damaging interpretation" (1987:71).

If the speaker does not have the possibility to avoid the FTA altogether or to use the "off record"-strategy, he will have to go "on record" - a strategy which is further divided into two subtypes. The "most direct, clear, unambiguous and concise [and thus face-threatening] way possible" (Brown/Levinson 1987:69) of these two subtypes is strategy number one, which works "without redressive action, baldly". Since this strategy involves the greatest amount of face-threat, it should be used only in circumstances which assure that the face-threat for the

Table 4. Brown/Levinson's five superstrategies for the mitigation of FTAs $(1987: 60,69)$

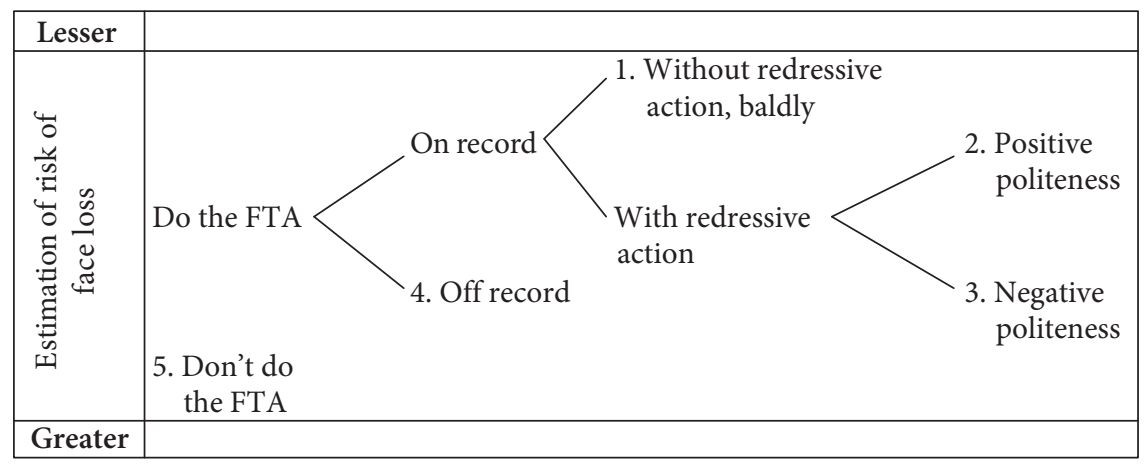


hearer is minimal. These include, for example, emergencies or time constraints, in which a speaker is forced to confine themselves to the most direct way of transmitting their message and where considerations for the other person's face are absolutely marginal:

A situation which combines all these external constraints would be making a "May Day" call from a foundering ship; this will certainly demand speaking with maximum efficiency. In emergencies or in highly task-oriented situations, such as teaching someone to drive, we find that the speaker is likely to focus on the propositional content of the message, and pay little attention to the interpersonal aspect of what is said.

(Thomas 1995: 170)

What is more, utterances can also be pronounced baldly when the power differential is so great, as, for example, between a boss and his employee or between a drill instructor and a recruit (see also Section 6.3), that the face-risk for the employee or the recruit is by the very nature of their relationship of no consequence, regardless of the rating of the imposition. Although going "on record" is an extremely straightforward way of articulating one's point of view, Brown/Levinson still ascribe certain payoffs to this kind of strategy since the speaker can, for example, "get credit for honesty, for indicating that he trusts the addressee; he can get credit for outspokenness, avoiding the danger of being seen to be a manipulator; he can avoid the danger of being misunderstood" (1987:71).

The second possible way for a speaker to go "on record" is "with redressive action", which includes "attempts to counteract the potential face damage of the FTA by doing it in such a way, or with such modifications or additions, that indicate clearly that no such face threat is intended or desired" (Brown/Levinson 1987: 69f.). Redressive actions of that kind can take one of two forms, depending on whether the positive or the negative face is in danger. Accordingly, Brown/Levinson distinguish between positive and negative politeness, the former being understood as the expression of solidarity, informality, and familiarity, which is supposed to emphasize the fact that both speakers want the same thing and have a common goal (Yule 1996: 62). Then again negative politeness, which is rated by Brown/Levinson as more polite than its positive counterpart, focuses on the addressee's negative face and consists of assurances that the speaker recognizes and respects the addressee's negative face wants and will not (or will only minimally) interfere with the addressee's freedom of action (Brown/Levinson 1987:70). Speakers therefore tend to show deference, emphasize the importance of the other's time or concerns, and even include an apology for the imposition or interruption (Yule 1996:62). In substantiating their five superstrategies, Brown/Levinson assign several substrategies to them, which are then illustrated with numerous examples. Similar to their 
implementations on FTAs, these kinds of lists can never be exhaustive and only provide some greater insight into otherwise purely theoretical categories.

The choice of an appropriate strategy is, in fact, based on a rather complex process of calculation in which the degree of face threat must be determined. Brown/Levinson (1987: 74ff.) suggest the equation $\mathrm{W} x=\mathrm{D}(\mathrm{S}, \mathrm{H})+\mathrm{P}(\mathrm{H}, \mathrm{S})+\mathrm{R} x$, thus assessing three basic sociological variables $(\mathrm{D}, \mathrm{P}, \mathrm{R})$ for the more or less conscious calculation of the weightiness of a particular FTA $(\mathrm{W} x)$ : the social distance (D), the relative power between the two interlocutors $(\mathrm{P})$ as well as the rank or size of the imposition of the act involved (R). While D tries to factor in the degree of familiarity and solidarity that exists between a speaker and their hearer, thus describing a symmetric, horizontal relationship based on the frequency and kind of past interactions, $\mathrm{P}$ measures the degree to which the speaker is able to impose their will on the hearer with a view to the power that the hearer has over the speaker. In this case, an asymmetric, vertical relationship exists between interlocutors. Finally, $\mathrm{R}$ is defined by culture and situation, since it accounts for the expenditures of goods and/or services the speaker is asking of the hearer, including the right of the speaker to perform the act, and the degree to which the hearer welcomes the imposition.

It is important to note, however, that we are not dealing with three completely independent variables, distinct and apart from each other, but with interrelated ones. Above that, these variables only matter "to the extent that the actors think it is mutual knowledge between them" (1987:74). In contrast to actors' assumptions of such ratings, actual power, distance etc. are in fact insignificant. These variables - due to their highly culture-sensitive nature - must be (re-)measured for every single instance of interaction. In Fraser's (1990:231) words,

$[\ldots]$ none of these variables can be viewed as a constant between individuals; participants vacillate in their social distance when job and anger intervene, relative power is altered as the roles and responsibilities change back and forth even over short periods of time, and the specifics of an act content or the circumstances of the participants at the time can easily cause a change in the ranking of degree of imposition.

Naturally, the Politeness Theory as outlined above received a lot of criticism ${ }^{32}-$ besides the fact that Brown/Levinson never give a definition of their key concept politeness even though it is a prominent part of the title of both their publications. The same holds true for the notion of universality, which was also heavily attacked

32. Among the most rigorous critics were probably Brown/Levinson themselves when they reviewed their 1978 paper in the introduction to their 1987 monograph, in which they commented on certain weaknesses of their approach, often put forward by fellow researchers. 
considering the fact that only a small corpus of linguistic data from three unrelated languages and cultures was used. In the following, only the most important points of criticism will be mentioned.

One of the most severe points of criticism is concerned with Brown/Levinson's view of FTAs as an almost ubiquitous phenomenon. This prompted Schmidt to state that their concept of politeness is "an overly pessimistic, rather paranoid view of human social interaction" (1980:104) since most speech acts are considered inherent FTAs in one way or another. Fraser carries this point to extremes when he remarks that theoretically all acts "require the hearer to do work to understand the speaker's communicative intentions" (1990:229). On the other hand, social beings that we are, we need contact and hence interaction with others, or, putting it rather extremely, we need to bother and be bothered by others. This would make FTAs the rule rather than the exception in our everyday lives. To solve this obvious contradiction, one could thus argue that a phenomenon as common as an FTA should simply be rid of its negative connotation.

A lot of criticism has struck one of the center pieces of Brown/Levinson's model: The five super-strategies and the politeness-ranking of these strategies. Blum-Kulka (1985) and others doubted, for example, whether off-record strategies are indeed always felt to be the most polite way of conveying say a request, since speakers might appear manipulative rather than polite. Above that, these strategies should not necessarily be seen as mutually exclusive, with positive and negative politeness leading the way.

The number of super- and substrategies did not remain uncommented either as it soon became obvious that "counting strategies is basically a fruitless exercise, since context is so important for interpreting the significance of any linguistic form" (Holmes 2006: 689). Consequently, the three sociological variables, power, distance and rank, took center stage. Although the inclusion of sociological variables in their model clearly was a much appreciated step in the right direction, their number did not remain undisputed. Many voices claim that these three "remarkably vague terms" (Werkhofer ${ }^{2} 2005$ : 175) fall well short of covering all influencing factors and/or that more of them need to be factored in - as, for example, the presence of a third/forth person. Brown/Levinson, however, elucidate that $\mathrm{P}$, $\mathrm{D}$, and R should not be understood as "the only relevant factors [as they simply] subsume all others (status, authority, occupation, ethnic identity, friendship, situational factors etc.) that have a principled effect on such assessments" (1987: 80). The authors also concede that "there may be a residue of other factors which are not captured within the P, D, and R dimensions" (1987: 16). Accordingly, the explanatory power of their equation is affected as well because it clearly oversimplifies the complexity of human relations. 
A false sense of simplicity is also seen - especially by more recent critics - in the misconception of the discursive nature of human interaction. Brown/Levinson have often been accused of not taking into account the discursive struggle over the social values of politeness - an accusation that should certainly be directed to the general modus operandi at that time than to this particular approach. In accordance with the proceedings of other approaches, Brown/Levinson base their analyses on the sentence-level and concentrate basically on the speaker (neglecting the hearer-perspective completely). Meaning-making in this model thus appears to be a unilateral and thus static endeavor. The opposite is, however, far more realistic: More recent approaches, leading to a regular paradigm shift (see Chapter 5), act on the assumption that the creation of meaning and mutual understanding should better be described in terms of a dynamic process in which participants negotiate meaning interactionally (Holmes 2006: 688).

\subsubsection{The predecessor: Goffman's classic approach}

Communication has many functions but an ever-present one is to put forth the kind of person one is and to suggest how one sees the other.

(Tracy 1990:217)

In 1967, eleven years before the first publication of Brown/Levinson's work, sociologist Erving Goffman presented his work about interactional order in human communication, Interaction Ritual. Essays on Face-to-Face Behaviour. This collection of (previously published) Goffmanian papers was to become extremely influential for the work of social science researchers as well as for linguists. ${ }^{33}$ Portioned in six separate essays, the first one of Goffman's reflections, "On Face-Work: An Analysis of Ritual Elements in Social Interaction", introduced the by now famous notions of face and face-work. ${ }^{34}$ Although proposing a theory of social interaction rather than a framework for polite behavior, this chapter nevertheless paved the way for Brown/Levinson's work about politeness and especially for their notion of face.

In identifying the subject of his account on social organization in everyday life interactions, Goffman writes: "It is that class of events which occurs during co-presence and by virtue of co-presence. The ultimate behavioral materials are the glances, gestures, positionings, and verbal statements that people continuously

33. A few years later, in 1979, a similar approach concerned with image work was proposed to the German-speaking academia by Werner Holly in his book Imagearbeit in Gesprächen. Zur linguistischen Beschreibung des Beziehungsaspekts.

34. Departing from Goffman's original spelling of face-work, the contracted form facework will be used in this study. 
feed into the situation, whether intended or not" (1967:1). In order to describe what he considers "behavioral materials", Goffman introduces his well-known terminology, starting with his understanding of a line, a notion which is closely linked to his concept of face. Thus, someone ${ }^{35}$ involved in social interaction behaves according to

a pattern of verbal and nonverbal acts by which he expresses his view of the situation and through this his evaluation of the participants, especially himself. Regardless of whether a person intends to take a line, he will find that he has done so in effect. The other participants will assume that he has more or less willfully taken the stand, so that if he is to deal with their response to him he must take into consideration the impression they have possibly formed of him.

(1967:5)

A line, one could therefore say, is the (un)consciously produced, mostly structured and perceivable way a person acts verbally and/or non-verbally in front of others. Since a speaker wants to convey a self-image, his (verbal) behavior, as expressed in his lines, is the condensate, so to speak, of whom he (thinks he) is and of whom he would like others to think he is. To that effect, face according to Goffman is

the positive social value a person effectively claims for himself by the line others assume he has taken during a particular contact. Face is an image of self delineated in terms of approved social attributes - albeit an image that others may share $[\ldots]$.

$(1967: 5)$

The concept of face, which originated in China, ${ }^{36}$ was known as early as the fourth century BC ( $\mathrm{Hu} 1944)$ by social anthropologists before it entered the scene of sociology. With regard to the line he has taken, a person "may be said to have, or be in, or maintain face" (Goffman 1967:6) when he behaves in accordance with his line - in his eyes and, even more importantly, in the eyes of the others. The typical emotional response, according to Goffman, would be feelings of confidence and assurance. On the other hand, a person "may be said to be in wrong face" (1967:8) in cases where his behavior can by no means be integrated into his line. Finally, a person "may be said to be out of face" (1967:8) in situations where he does

35. Goffman's ideal social actor is also based on a Western model of interactant (BargielaChiappini 2003: 1463).

36. Chinese scholars share Goffman's point of view in stressing that face is a situational construct which is firmly embedded in interpersonal relations (Ho 1994). Goffman's adoption of the Chinese notion of face bears a weak point, though: Originally devised to account for cultures putting group identity before individual identity, Goffman removed this concept from its original context and transported it into the clearly individualistic Western hemisphere (cf. Vilkki 2006: 326ff.; Bargiela-Chiappini 2003). 
not even dispose of a line that would be expected of him in this sort of contact. The latter two cases are likely to trigger feelings of embarrassment, shame and inferiority. Speakers may also feel disappointed and confused because of failed expectations: An encounter which was intended to strengthen face may have effectuated the exact opposite. Consequently, a person may become "shamefaced", as Goffman (1967:9) calls the emotional outcome of this worst case scenario. In the end, a person could even "lose his face". The only way out in this kind of situation is what Goffman (ibid.) calls "to save one's face", referring to the process by which someone sustains an impression for others that he has not lost face. ${ }^{37}$

In this play with faces, Coupland/Jaworski (2004:22) even see a "dramaturgical element of every day encounters", in which speakers as 'actors' do not only talk but actually 'perform'. Face, consequently, resembles a 'stage mask' "that people carefully select and 'wear' to conjure up specific images and effects". The dynamism of this performance is owed to the multiple roles each participant disposes of and to the fact that he is emotionally attached to the image that others associate with him. Accordingly, a person reacts immediately when perceiving even subtle changes in the face he is "given" by his interlocutors. Then again, in encounters with people whom he will probably never have dealings with ever again, his line is of minor importance since his behavior remains without further consequence for his future face, which is attributed but only existent for the short time of this particular encounter. His self-image, thus, stays unaffected.

These lines of thought already indicate that there is in fact an important connection - especially in terms of face creation and modification - between the individual (and his face) and the influence of other participants (and their faces) that must not be underestimated. For this reason, Goffman (1967:7) claims that "a person's face clearly is something that is not lodged in or on his body, but rather something that is diffusely located in the flow of events in the encounter". He thus considers face to be a dynamic, almost ephemeral notion:

In any case, while his social face can be his most personal possession and the centre of his security and pleasure, it is only on loan to him from society; it will be withdrawn unless he conducts himself in a way that is worthy of it. Approved attributes and their relation to face make of every man his own jailer; this is a fundamental social constraint even though each man may like his cell.

(1967: 10)

Accordingly, face is not (only) something that we can determine in and for ourselves by the choice of lines. On the contrary, we very much depend on our

37. The capacity to suppress and conceal any tendency to become shamefaced is what Goffman calls poise (1967:9), which helps people control their embarrassment and hence the subsequent embarrassment that they and others might experience over their embarrassment. 
interlocutors to "give" us face. To put it in a nutshell, we are what and how others think we are. This rationale, however, opens up another can of worms in form of the old, baffling, and largely unanswerable question of whether there is a true self that exists apart from interaction. Above that, face is a conglomerate of a multitude of different, highly situation-specific facets of face, each being appropriate only in certain situations, which is restructured and reinvented through every new communicative encounter. Goffman (1967:31) therefore proposes a double definition of the self as "an image pieced together from the expressive implications of the full flow of events in an undertaking" on the one hand and "as a kind of player in a ritual game who copes honorably or dishonorably, diplomatically or undiplomatically, with the judgment for contingencies of the situation". Face is thus a product of the interactional dyad, not a fixed inner possession, which lies "in the eye of the beholder". Thus, Goffman's notion of face "is a socially attributed aspect of self that is temporarily on loan for the duration of the interaction in accordance with the line or lines that the individual has adopted" (Watts 2003:125).

In Goffman's model, the hearer is allocated a central position: Not only has he major influence on the formation of face in the speaker's mind, but above that, he clearly has a face of his own to gain or to lose:

Just as the member of any group is expected to have self-respect, so also he is expected to sustain a standard of considerateness; he is expected to go to certain lengths to save the feelings and the face of others present, and he is expected to do this willingly and spontaneously because of emotional identification with the others and with their feelings.

(Goffman 1967: 10)

Motivated by different reasons, participants in social interaction are thus concerned with the maintenance of their own as well as their interlocutors' faces, which is why "tacit cooperation will naturally arise so that the participants together can attain their shared but differently motivated objectives" (Goffman 1967:29). Therefore, a person who does not want to appear heartless or shameless should always attend to his interlocutor's face needs. As a side benefit, he avoids having to witness the other's humiliation or his defacement (1967:11). The mutual care for interlocutors' faces, which Goffman calls facework, is a basic structural feature of interaction in general, and even more so of face-to-face interaction. The "maintenance of face is thus a condition of interaction, not its objective" (1967: 12). Facework can be seen as interlocutors' (un-)conscious actions, which can become habitual:

By face-work I mean to designate the actions taken by a person to make whatever he is doing consistent with face. Face-work serves to counteract 'incidents' - that is, events whose effective symbolic implications threaten face.

(1967: 12) 
According to Goffman, facework can exhibit a defensive or a protective orientation. While the first one aims at defending one's own face, the latter seeks to protect the interlocutor's face. Although clearly distinguished terminologically, practices to protect either of those faces can, in fact, be taken simultaneously. As Goffman explains, "[i]n trying to save the face of others, the person must choose a tactic that will not lead to loss of his own; in trying to save his own face, he must consider the loss of face that his action may entail for others" (1967: 14). In terms of tactics to save one's interlocutor's face, Goffman invokes avoidance and corrective processes. While the former subsumes a range of maneuvers, which shall avoid the occurrence of face threats in the first place, the latter becomes operative when a face threat could not be prevented by the participants and cannot be overlooked either. Consequently, "participants are likely to give it accredited status as an incident" and take measures to rectify it because "one or more participants find themselves in an established state of ritual disequilibrium or disgrace, and an attempt must be made to re-establish a satisfactory ritual state for them" (Goffman 1967: 19).

With facework being the default in interpersonal behavior, Goffman argues that "there is no occasion of talk so trivial as not to require each participant to show serious concern with the way in which he handles himself and the others present" (1955:226). Although critical voices accuse Goffman of designing a social actor who is "almost obsessively concerned with his own self-image and selfpreservation" (Bargiela-Chiappini 2003: 1463), it is indeed realistic to assume that "there is no faceless communication" (Scollon/Scollon 1995:38) and that speakers have some sense of facework and experience in its use at their command. Being, however, the multilayered undertaking that it is, the choice of appropriate facework presents considerable problems even for experienced interlocutors. In both mediated and immediate encounters (Goffman 1967:33), usually persons already stand in some kind of social relationship with one another and expect to stand in a given relationship to them after the particular encounter has ended (Goffman 1967:41). For that reason, Goffman claims that interlocutors during encounters mainly make an effort "to get through the occasion and all the unanticipated and unintentional events that can cast participants in an undesirable light, without disrupting the relationships of the participants" (1967:41). Facework is not necessarily transmitted explicitly and sometimes, more subtle ways of conveying facework seem advantageous as it

often relies for its operation on a tacit agreement to do business through the language of hint - the language of innuendo, ambiguities, well-placed pauses, carefully worded jokes, and so on. The rule regarding this unofficial kind of communication is that the sender ought not to act as if he had officially conveyed the 
message he has hinted at, while the recipients have the right and the obligation to act as if they have not officially received the message contained in the hint. Hinted communication, then, is deniable communication; it need not be faced up to.

(Goffman 1967:30)

Finally, Goffman (1967:24) points out that facework can also be exploited strategically, a tactic he calls aggressive use of face-work. Someone can, for example, adopt a particularly modest line, hoping that this behavior will gain him a compliment - a tactic which is also known as fishing for compliments. Here, facework becomes "less a scene of mutual considerateness than an arena in which a contest or match is held. The purpose of the game is to preserve everyone's line from an inexcusable contradiction, while scoring as many points as possible against one's adversaries and making as many gains as possible for oneself" (Goffman 1967:24). It is obvious that Goffman also kept in sight the flip side of the coin of consideration for others, namely the negative, manipulative side of facework, which will be dealt with in detail in Chapter 5 .

\subsubsection{Brown/Levinson vs. Goffman: Some interrelations}

Both approaches being introduced, we can shed some light on the interrelatedness of Brown/Levinson's and Goffman's theories. First of all, let us juxtapose the two notions of face presented above. As we have seen, Goffman had in mind a social concept of face which is dynamically negotiated between interactants in contact. It is bestowed post-factum from the outside for the duration of the exchange, which makes face an instable concept and subject to situation-specific variation (cf. Watts 2003:104). Face is thus not only constituted like a piece of patchwork with center pieces and more peripheral ones, but also partly reinvented within every ongoing talk exchange over longer stretches of time. Therefore, it is a summative construct with more or less changing constituents. Due to its permeable borders that allow for influences from the outside to pass through, the processes of change are triggered by interlocutors, who, in turn, can be biased by the speaker's (non)verbal behavior. It is this behavior which may support or contradict the speaker's line and may have a very strong influence on future alterations and ratifications of his face - again induced by his interlocutors. This social interplay of constant alterations and readjustments of a speaker's face can be visualized in a circular model (see Figure 11).

Brown/Levinson, on the other hand, assume a rather individualistic concept of face which is bestowed as a pre-facto and consistent desire from the inside (cf. O’Driscoll 1996:6). Rather insusceptible for outside influences and hence independent of social interaction, face can be seen as a binary construct, often 


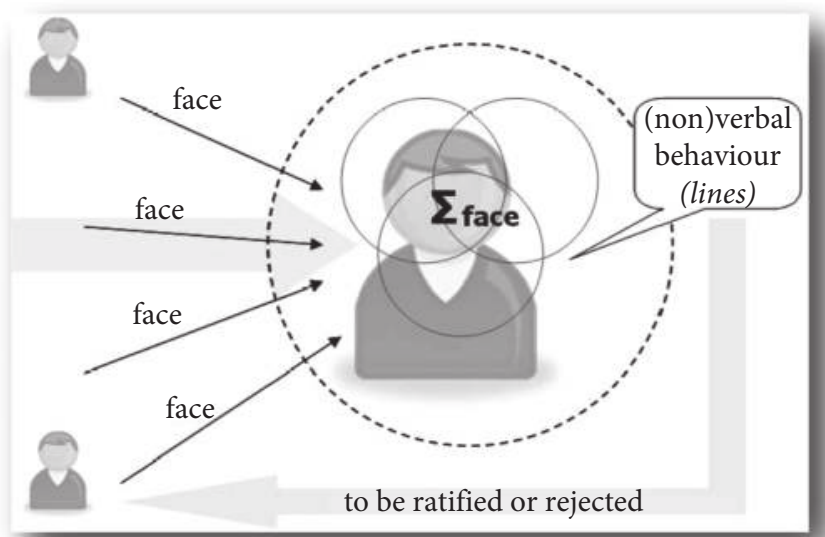

Figure 11. Schematic representation of Goffman's notion of face

entitled as face-dualism, consisting of two innate wants, mirroring basic human needs: The need to be independent and the need to be an accepted part of the group (negative and positive face). The two authors see face not as a socially created, interpersonal reality, but as intrapersonal wants of the model person (cf. Werkhofer $\left.{ }^{2} 2005: 168\right)$. The particular filling of these two abstract needs is determined individually by each and every speaker in each and every culture and context. Accordingly, Brown/Levinson's notion of face is a ready-made self-image, constructed prior to the interaction, which shall be upheld by society. Insights into the speaker's understanding of face and his personal face needs can only be gained from subjective evaluations of the speaker's (non)verbal behavior.

In lacking a pivotal aspect, the interactive dimension, Brown/Levinson's idea of face indeed differs considerably from Goffman's sociologically richer model: "Brown and Levinson seem to be thinking of the self as a stable core of values lodged somewhere in the individual, whereas for Goffman self is far less 'real' and is constantly renegotiable" (Watts 2003:105). Critics hence considered Brown/ Levinson's concept of face as extremely selective and far too static, "abstracting not only from the dimension of ritual order, but from all kinds of social order" (Werkhofer ${ }^{2} 2005: 178$ ). This becomes evident when depicted graphically in the following unidirectional model (see Figure 12).

Although Brown/Levinson's idea of positive face seems to be derived directly from Goffman's understanding of the term, the same cannot be said about their notion of negative face. We can, however, detect a correspondence between positive politeness and Goffman's avoidance rituals, if one neglects the different targets - the hearer (Brown/Levinson) and the speaker (Goffman) - for just 


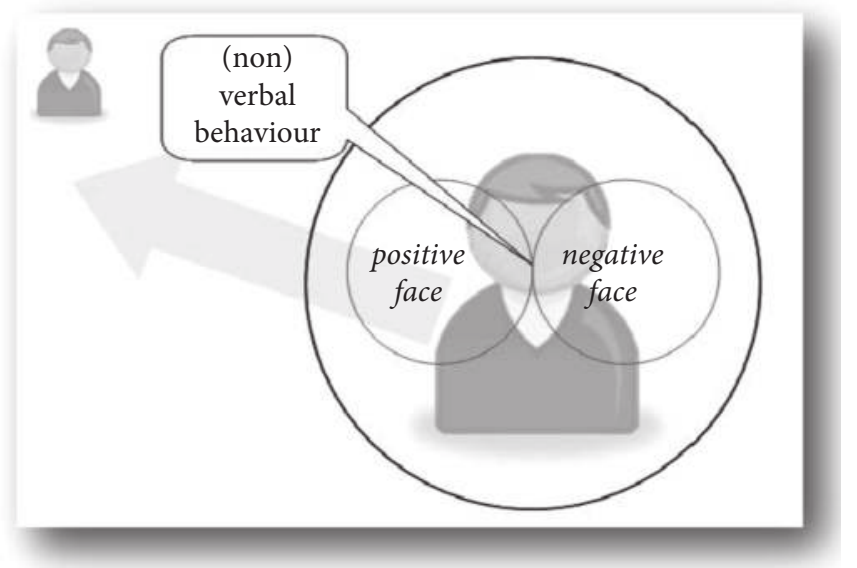

Figure 12. Schematic representation of Brown/Levinson's notion of face

a moment. Both strategies aim at saving face: Positive politeness tries to claim common ground between participants and attend to each other's needs, whereas avoidance rituals try to steer clear of a possible lack of common ground and thus of face challenges as such.

Besides the two different notions of face, there is still a second important question to be answered: What kind of relationship exists between Goffman's notion of facework and Brown/Levinson's concept of politeness? Or in Watts' words: "Is it not the case that the strategies suggested by Brown and Levinson [...] are facework strategies rather than politeness strategies?" (2003:93) - a question which is rather difficult to answer considering the fact that we are still in the dark about Brown/Levinson's understanding of politeness. What we can, however, proceed from is the assumption that politeness, at least in the eyes of Brown/Levinson, strongly equals face-threat-mitigation. As we have seen in the explanations above, politeness is used in their model as a strategy to "soften the blow" for the faces of everyone involved. The sub-strategies proposed by Brown/Levinson to accomplish this goal exhibit, however, a serious structural problem in mixing analytical levels, i.e. by giving behavior-related as well as purely grammatical instructions. Surely, the general idea is not wrong, since politeness is mirrored by more than just grammatical structures in actual language usage. Still, the question remains: What do they actually describe, facework or politeness? We could go as far as ascribing their behavior-related instructions (such as seek agreement) to the sociopsychological field of facework, while its purely linguistic condensate (strategies as, for example, impersonalize $S$ and $H$ ) could be put under the umbrella term of 
linguistic politeness. ${ }^{38}$ To quote Holtgraves, linguistic politeness could then "be viewed as essentially the linguistic means by which face-work is accomplished" (2005:75). Leaving aside the admittedly blurred boundary between these two fields, one can nevertheless outline two general strands of approaching the topic: Socially appropriate behavior - based on a speaker's rational stance as well as his social beliefs, motives and goals - and its realization in linguistic surface structures.

This distinction harbors another serious problem, though. Watts certainly has a point when he doubts the usefulness of a term such as linguistic politeness, which "runs the risk of becoming vacuous, since any linguistic expressions and all which are used in face-maintenance would then be examples of 'linguistic politeness"' (2003: 118). Accordingly, utterances which are labeled as linguistically polite - the typical example being particles such as please and thank you - can, but do not necessarily need to be used for facework, let alone be received as such by interlocutors. And vice versa: Utterances which are obviously not polite on the surface, such as you silly bugger or even swear words, can nevertheless be employed for facework (cf. Watts 2003:93).

In default of a definition of politeness, one could therefore argue that Brown/ Levinson's ideas indeed orbit around what Goffman called facework rather than politeness, although most certainly with a narrowed scope because it is "dealing only with the mitigation of face-threatening acts [and fails to] account for those situations in which face-threat mitigation is not a priority, e.g., aggressive, abusive or rude behavior" (Locher/Watts 2005:10). While politeness, according to Brown/Levinson, operates only when face interests are at stake, facework has a much wider scope and "can be used to defuse, manage, enhance, or downgrade self and/or other's face" (Ting-Toomey 1994:2). Since politeness and facework intersect only partially, with politeness being only one aspect of facework, politeness theory can never be fully equaled with face theory (Watts 2003:117; cf. Bargiela-Chiappini 2003: 1463; Kasper 1997:377).

38. Note, however, that the notion of linguistic politeness is also used heterogeneously, since it can mean the use of verbal means to translate a polite attitude on a textual level but also the study of politeness as conducted by linguists in general. In the following, I will use the term linguistic politeness in the first of the two senses. 


\subsection{Summary: What to keep and what to drop}

The critical introduction to some of the classic approaches to politeness has shown that the linchpin of models trying to get a sensible grip on a fluctuating notion such as politeness is - at least for this investigation - their applicability to actual data as well as the logical consistency of their internal structure. While the rather static approaches proposed by Lakoff and Leech were certainly necessary and useful forerunners for present-day research on politeness, they are clearly not dimensioned for an analysis of interpersonal relations in message boards, since they cannot do justice to the dynamic nature of communication. This is partly due to the fact that both models were built on speech act theory and Grice's CP, treating communication as a rationalistic and objectifiable phenomenon. Above that, speakers appear as central figures whose intentions can be reconstructed faithfully by hearers. Most of the time, this is even done with an extremely limited scope on (usually very complex) contextual variables. Besides the fact that utterances are ascribed single functions, both approaches neglect the negotiation and co-construction of meaning and evaluation of politeness.

A viable theory must take account of all those factors that are elementary to the interactive negotiation of meaning within human communication, most of all situation-specific contextual aspects. Consequently, we need to fathom as many of the contextual factors that are accessible in and relevant for the strings of conversation in our message board corpus as possible (see Chapter 6). As a result, the static approaches will be left behind. The social norm view, including Fraser's conversational contract view, should, however, not be discarded that easily.

The most important input for the upcoming line of thought surely concerns key concepts such as face, facework and politeness. The initial insight that the latter of the three notions should not be placed on the same analytical level as facework will be further elaborated, once politeness will be embedded within a larger framework (cf. O'Driscoll 2011). With facework being a comparatively straightforward and unproblematic notion, designating the negotiation and mutual care for interlocutors' faces, all there is to do is to agree on an understanding of face, having in mind the two propositions by Brown/Levinson and Goffman with their strengths as well as their weaknesses. In short, Goffman's model clearly underestimates the human nature of individuals. Then again, Brown/Levinson's model hardly accounts for the social nature of human interaction. Since a realistic model should combine both aspects, a "best-of-both-worlds" model is called for (see Figure 13). In this combined model, we take Goffman's circular, social view as a springboard and enrich it with a valuable element from Brown/Levinson's model, the idea of inherent, universal human needs of association and dissociation. We 


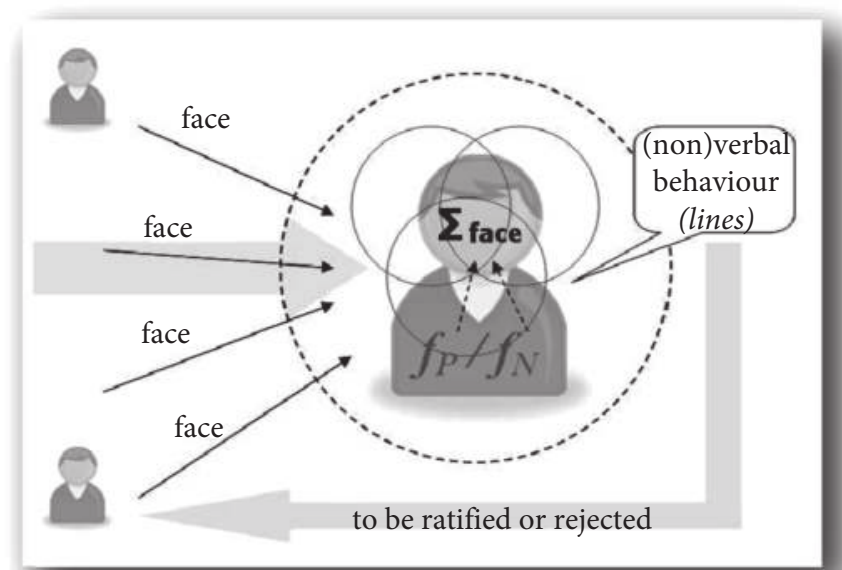

Figure 13. Schematic representation of a combined model of face

will keep their idea of positive and negative face because obviously, "face dualism is just too valuable to be jettisoned" (O’Driscoll 1996:4). According to this model, face is negotiated socially and reconstructed constantly in every instance of social encounter, but always on the basis of immanent human desires, which Brown/Levinson called positive and negative face $\left(f_{P}\right.$ and $\left.f_{N}\right)$. Although those two factors clearly influence the construction of face, we need to remember that their individual nature differs from person to person. As a matter of fact, face is a summative conglomerate, which (re-)constitutes itself in interaction and which is composed of numerous different and partly ephemeral facets. Depending on the situation speakers find themselves in, they activate and sometimes even create a unique constellation of these facets, which form the face speakers consider appropriate for a certain encounter and which they display through their (non)verbal behavior (cf. Goffman's lines). In doing so, speakers must have a great deal of rather stable facets at their disposal, which have grown with them, so to speak, reflect their biography and past experiences and have proven to be successful in previous interactions (cf. Locher 2011; O'Driscoll 2011). If this were not the case, individuals would have to reinvent themselves constantly, which would at best be extremely time-consuming and strenuous. At worst, however, this lack of consistency in our personality would look pathological as in the case of multiple personality disorder.

Finally, the reactions and assessments of interlocutors will show speakers whether they "put on the right face" or whether they need to change their lines to 
yield the desired effect in terms of their face. Even Brown herself later shared this interactive view on face, claiming that

B\&L face wants are an interactionally relevant phenomenon, not a matter of our deepest personality and identity construction [because] they are only attributed to a person who is constructing his/her utterance in an interactional context. [Consequently,] face is indisputably interactionally created and manipulated.

(Arundale 2006: 199f.; cf. Brown 2001: 11623) 



\section{Interpersonal relations II}

\section{Putting (im)politeness in an integrative perspective}

\subsection{Introduction}

Still in search for a viable definition and way of approaching the notion of politeness, this chapter delves into present-day discussions of politeness, thereby focusing on the so-called postmodern approaches. More importantly, though, it aims to see the bigger picture of interpersonal relations by treating politeness for what it is, namely as one ingredient of a comprehensive framework of several related phenomena. In feeling out said framework, this chapter will also lead us to the "dark side" (Austin 1990) of interpersonal relations and to the realm of impoliteness.

Admittedly defined rather vaguely, postmodernism is used as an umbrella term for those approaches which are "grounded in a broadly constructionist position on the nature of reality" (Haugh 2007:297), including, of course, the dynamic endeavor of creating meaning. It is thus out of the question to perceive language use and especially politeness in terms of the infamous conduit metaphor (cf. Reddy 1979), where ready-made linguistic end products are encoded by the speaker, transported and decoded by the hearer. As human communication is far more complex than that, there can be no such thing as ready-made end products, which are transported mechanically and unambiguously to our interlocutors, especially not when it comes to the construction of meaning (cf. Arundale 2006: 195; Bublitz 2009: 40ff.). Viewed from a postmodern perspective, advocated by pragmaticians such as Eelen (2001), O'Driscoll (2001), Watts (2003, $\left.{ }^{2} 2005\right)$, Locher $(2004,2006 a, b)$, Locher/Watts $(2005,2008)$ as well as by Spencer-Oatey $(1993,2002,2005,2007)$, politeness is apprehended as evolving and emerging dynamically during interaction. As the following example illustrates, all conversational action is - as Arundale $(2006,2010)$ calls it - "conjointly co-constituted":

[A] ssume a first speaker utters "That's a nice jumper". If the second utters "You can't borrow it", the two together interactively achieve operative interpretings of the first utterance as a request. If the second speaker were to say "Thanks", the two would conjointly co-constitute operative interpretings of the first utterance as a compliment.

(2006: 196) 
In the following, politeness as well as impoliteness are put in perspective, this time, however, from an integrative and discursive point of view. In order to do so, Locher/Watts' (2005) framework of relational work will be at the center of attention.

\subsection{A working definition of politeness}

Nothing will ever fix the reference of "politeness" to human behavior once and for all.

(Sell ${ }^{2} 2005: 113$ )

Why should one select a statement as daunting and as discouraging as Sell's introductory quote as the starting point for a chapter which intends to do just the seemingly impossible, finding a definition for a quality of interaction which is subject to change through time and across cultural space? The answer is rather simple: because he is right. As will be shown in the following, the search for a consensual definition of a term as complex as politeness is bound to fail, which is why we can at most find a tailor-made, approximate working definition ${ }^{39}$ to serve as a basis for the targeted analysis of interpersonal relations in the online message board at hand.

The search for a definition of politeness is intertwined with a major methodological decision: Either we approach the notion of politeness with a purely abstract model in mind, hoping for the emergence of some universally valid mechanisms, which can be applied to each and every particular instance. In the worst case, however, such an abstract model yields findings that are so general that they will only be of a limited informative value. Alternatively, we dispense with a heightened degree of abstraction in favor of an investigation of a reduced set of individual cases. Although findings are far from universally valid, they are still significant and informative, at least for the limited scope of investigation. It is indeed the latter course of action that will be picked for the empirical analysis of this study.

This methodological predicament is partly mirrored, and most of all named, in Watts et al.'s (2005) differentiation between first-order politeness and second-order politeness - a dichotomy which was later taken up by Eelen (2001), who termed it politeness ${ }_{1}$ and politeness ${ }_{2}$. Watts et al. explain that the "pursuit of universals will necessarily involve us in second-order concepts, whereas the investigation into politeness in individual cultural frameworks will almost inevitably involve

39. See Watts (2003:51f.) for an interesting discussion concerned with the definition of politeness based on an exhaustive compilation of numerous previous definitions. 
first-order concepts" (2005:4). Accordingly, first-order politeness focuses on a common-sense, folk, or lay understanding and evaluation of social behavior as politeness and deals with questions such as "How do members of the community perceive and classify action in terms of politeness?” (Kasper 1997:375). Secondorder politeness, on the other hand, is an abstract, theoretical construct within a top-down model of language usage (Watts et al. ${ }^{2} 2005: 3$ ). Note that only the latter direction involves claims of universality.

In pursuing a first-order approach to politeness, researchers can only hope to find sporadic testimony of interlocutors' individual, atomistic, group-specific evaluative snapshots in current situations on which to base their theorizing. As only those basic tendencies are in fact tangible and hence suitable starting points for investigations, I agree with Watts, ${ }^{40}$ who points out that "investigating firstorder politeness is the only valid means of developing a social theory of politeness [because] second-order politeness should concern itself with the discursive struggle over first-order politeness" (2003:9). In this sense, observations from the domain of first-order politeness can at least give some indication of the concept of second-order politeness. Only this way can social scientists avoid lifting the term politeness out of the realm of everyday discourse and elevate it to the status of a theoretical concept. Based on some evaluative specimens, researchers still tried - and failed - to abstract away from them in search of a second-order grip on politeness. This failure can be chalked up to the fact that

the first thing we would have to do is to find ways of isolating across cultures all those strategies, verbal as well as nonverbal, that construct, regulate and reproduce forms of cooperative social interaction - an obviously impossible task.

(Watts 2003:49)

For this reason, the focus of research must be and has always been, consciously or not, on first-order politeness because the only thing we can actually do is to study how individuals evaluate and struggle over first-order politeness. In so doing, native speakers rely very much on the "feel for the game" (Watts 2003:75), which they develop continuously through the participation in a wide variety of interactions. Clearly, this kind of culturally determined experience evades being wrapped into rules.

Following Locher/Watts' advice "to take native speaker assessments of politeness seriously and to make them the basis of a discursive, data-driven, bottom-up approach to politeness" (2005:16), a first explorative analysis of the corpus material at hand (cf. Section 7.2) proves insightful in terms of participants' explicit

40. In this context, Watts makes it perfectly clear that his 2003 monograph is to be understood as a "radical rejection of politeness" ${ }_{2}$ (2003: 11). 
first-order conceptions of politeness. In addition to the code of conduct described in Section 3.3.2, this procedure provides the second clue to message board users' understanding of this particular term - or at least to some of the evaluative snapshots mentioned above. It is, however, hardly surprising that lexemes such as polite or impolite are very rarely if ever explicitly used for evaluations of interactants' behavior (cf. Watts 2003:218). Indeed, only six instances of users mentioning the lexemes polite or politeness at all in the entire corpus (my emphasis) could be found:

(1) $[\ldots]$ a massive change from the kind-hearted, polite boy he was when I knew him not so long ago $[\ldots]$

(cpj1987, thread \#10: post 47)

(2) [...] If I walk past someone in the street and we make eye contact then I smile out of politeness, regardless of their gender - its jsut a friendly gesture.

(doodle7, thread \#14: post 5)

(3) $[\ldots]$ i think if there are two people walking past each other on a lonely street its awkward not to smile or look at each other! so usually its just out of politeness or being friendly id say.

(Jdizzle09, thread \#14: post 44)

(4) [...] The guy at Chicago was perfectly polite, if a little fed up (but who wouldn't be doing that job?). [...] The guy at LAX when I went in 2005 was really polite, so it's a mixed bag in that respect. [...]

(JSS16, thread \#26: post 81)

(5) [... I'm not insecure, I don't even know who you are, I just don't really see any reason why I need to be espescially polite or civil towards you. [...]

(bete noire, thread \#42: post 58)

(6) [...] After months of me waiting to get a reply I emailed them asking what was taking so long (fairly politely) only to be told that [...]

(LastLordofTime, thread \#47: post 25)

In most of these examples, the search terms are used to describe actions (example (6)) or persons (examples (1) and (4)) within a user's narration of events - unfortunately without letting us know, why these actions and persons deserved the label polite. Since examples (2) and (3) give at least one instance of politeness (smiling when walking past someone on the street), we learn about these users' assessments of these specific situations. The third and the fifth example are interesting insofar as polite is mentioned in the same breath as being friendly and civil. The author of example five even explicitly doubts the necessity of the two qualities for Internet exchanges.

As predicted, we cannot learn nearly enough from these scarce examples to deduce a first-order definition of politeness in this CoP. Two conclusions must be 
drawn from this first empirical endeavor, which are also mirrored in the analytical setup in Chapter 8. (1) More often than not, we are lacking explicit participants' evaluations, which is why we need to take the dyad of the communicating partners as the minimum unit of analysis and focus on complete series of moves and counter-moves. Only this way can we hope to witness a discursive struggle over (first-order) politeness and related phenomena. Although we will definitely keep our eyes open for explicit evaluations, interlocutors' implicit reactions will be assigned a prominent, since hopefully expressive role in this process. (2) For those cases, in which hearer reactions are not as revealing as expected, we should take back-up measures and pursue a second-order line of argumentation for a consistent evaluation of politeness from an outside perspective. To avoid entering the empirical analysis empty-handedly, I propose the following second-order working definition of politeness, which tries to capture this opaque phenomenon the best way possible:

From the speaker's perspective, politeness is rational because purposeful (non-) linguistic behavior, which (un)consciously aims at maintaining social order by showing consideration for others. Based on experience, it exhibits an individualistic and mental nature. From a social perspective, it is negotiated collaboratively in concrete interaction. Strongly dependent on contextual factors, politeness must be considered an evaluative and instable notion.

In a nutshell, being polite means reciprocally showing "consideration for others, often at the expense of one's own interests" (Watts 2003:31), but always with a view to successful present, sometimes even future interaction. Thus, for the time of the actual exchange, participants pursue a common (communicative) goal. They do so either unconsciously by means of ritualized, fixed expressions, or, alternatively, by deploying politeness creatively and idiosyncratically, often in a deliberate and goal-oriented fashion. In any scenario, politeness can be transmitted through a wide range of communication channels, with language being only one such channel.

\subsection{Locher/Watts' comprehensive framework of interpersonal relations}

In fact, politeness is not the only discursively constructed entity, which arises out of actual interaction. The same holds true for related notions such as impoliteness, over-politeness and the like, which is why Locher/Watts (2005) propose a comprehensive framework of what they call relational work. It incorporates the entire spectrum of interpersonal relations, yet tries to set its components apart from each other. Before we can, however, delve into the inner make-up of this 
framework, the very notion of relational work needs defining. According to Locher/Watts, it is

the 'work' individuals invest in negotiating relationships with others. Human beings rely crucially on others to be able to realize their life goals and aspirations, and as social beings they will naturally orient themselves towards others in pursuing these goals.

(2005: 10)

Three years later, Locher/Watts specify their initial definition insofar as relational work includes "all aspects of the work invested by individuals in the construction, maintenance, reproduction and transformation of interpersonal relationships among those engaged in social practice" (2008: 96). It is no coincidence that this definition is reminiscent of Goffman's notion of facework. In fact, Locher/ Watts are very clear about the fact that Goffman's work in general and his idea of face in particular (see Section 4.3.2) is the driving force behind their own approach. ${ }^{41}$ Locher even goes as far as using the terms relational work and facework synonymously, claiming that the "process of defining relationships in interaction is called face-work or relational work" (Locher 2004:51). In this regard, I beg to differ. Though it is certainly true that both notions are dependent on negotiation processes, are always present in any form of socio-communicative encounter and are definitely intertwined, they still differ in terms of their focal point. In retrospect to Goffman's original definition of facework as "the actions taken by a person to make whatever he is doing consistent with face" (1967:12), interlocutors orient to each others' faces and negotiate face claims. Then again relational work, true to its name, foregrounds the negotiation of present and future relationships between individuals (cf. O’Driscoll 2011 for the related differentiation between face and politeness).

Among the string of nouns used in their second definition to relational work, one strikes as particularly characteristic of Locher/Watts' integrative approach. As a matter of fact, the noun transformation hints to the fact that relational work is not only oriented to the maintenance of harmony, cooperation, and social equilibrium (2005:11). Quite to the contrary relational work also touches on the flip side of the coin, as it "comprises the entire continuum of verbal behavior from direct, impolite, rude or aggressive interaction through to polite interaction, encompassing both appropriate and inappropriate forms of social behavior" (Locher 2004:51). While Brown/Levinson distinguish between polite and impolite behavior, the umbrella term relational work clearly subsumes a much wider panoply of

41. Although Locher/Watts (2008:96) mention that they also include Brown/Levinson's perspective on face in their understanding of relational work, they do not give any detail on how they understand these two concepts of face to be actually united (but see Section 4.4). 
Table 5. Framework of relational work (Locher/Watts 2005: 12, adapted)

\begin{tabular}{c|c|c|c}
\hline \multicolumn{4}{c}{ Relational work } \\
\hline negatively marked & unmarked & positively marked & negatively marked \\
\hline impolite & non-polite & polite & over-polite \\
\hline inappropriate & appropriate $^{42}$ & appropriate & inappropriate \\
\hline
\end{tabular}

forms of negotiating interpersonal relations. The focus is thus widened far beyond the fuzzy limitations of politeness, which is now seen as one cog in the wheel within the larger framework of relational work as outlined in Table 5. As indicated in the first horizontal row, Locher/Watts differentiate between unmarked and (positively or negatively) marked behavior. It is marked negatively in those cases where it appears to be inappropriate in the eyes of the interlocutors (see last horizontal row). Participants can come to this negative evaluation when they perceive their interlocutor's behavior to be downright impolite (see the first column) or over-polite (see the last column). ${ }^{43}$ On the other hand, positively marked behavior, as displayed in the black-rimmed column, strikes as being distinctly polite and hence appropriate. Locher/Watts (2005: 14), however, distinguish very carefully between this type of appropriate behavior and a second one: As indicated in the second column, unmarked behavior also counts as appropriate, although non-polite behavior. To illustrate this distinction, Watts (2003:257) invokes the following scenario:

Imagine that you have booked two tickets to see a play and that they are numbered P51 and P52. Twenty minutes before the play is due to begin you locate row $\mathrm{P}$ and move along it to seats 51 and 52 only to find that someone else is already sitting there. What is the appropriate mode of behaviour in this situation?

Watts (ibid.) lists several options for verbally calling attention to the fact that those sitting in seats P51 and P52 must have made a mistake:

42. As a synonym for the term appropriate, Watts (2003:19) proposes the term politic to label interlocutor's evaluations of "[1]inguistic behavior which is perceived to be appropriate to the social constraints of the ongoing interaction, i.e. as non-salient”. Although he sees an advantage in this second-order concept "precisely because it is not in common usage and indexes a wide variety of forms of social behavior that include but are broader than what might be referred to in lay terms as POLITE" (2005: xlii), the notion will be rejected for this study.

43. Since both of these two types of relational work have the same effect in terms of the interlocutor's evaluation, Watts (2005: xliii) represents the same content in a circular shape in which both ends of Table 5 actually meet. The advantage of this form of representation is obvious: Impolite and over-polite behavior appear right next to each other, indicating their relatedness. 
Excuse me. I think you're sitting in our seats.

Excuse me but those are our seats.

I'm sorry. I think there must be some mistake.

I'm sorry, but are you sure you've got the right seats?

All of these four statements seem to be appropriate to resolve this situation. Whether one or all of them can also be considered polite, is, however, totally open to discussion. In this case, I side with Watts, who argues that we are dealing with cases of unmarked, non-polite/appropriate behavior which "can be expected in this type of situation [since] there's not much else you can say in" (Watts 2003:257, original emphasis). Let us not forget, though, that all kinds of indirect speech acts, such as "How strange, you seem to have the same tickets as we." offer lots of creative alternatives to Watts' utterances.

A differentiation between these two types of appropriate behavior is called for, since a great deal of relational work actually is of an unmarked nature and goes largely unnoticed (Locher/Watts 2005: 11). Non-polite behavior thus encompasses all those instances of appropriate behavior which do not excel through being explicitly polite. As a consequence, the scope of politeness is limited considerably. Summarizing the most important insight of their distinction, Locher/Watts affirm that "polite behavior is always [appropriate] while [appropriate] behavior can also be non-polite" (2005:12). In other words, not everything that is appropriate in a certain situation necessarily earns the label polite. For an utterance to be estimated as polite, there must be a certain excess or surplus, which lets it stand out positively in contrast to other appropriate utterances and which encompasses behavior that is perceived to be more than is socially required. For obvious reasons, this perspective has been termed the surplus approach to politeness repeatedly (cf. Kasper 1990; Locher 2004; Watts ${ }^{2} 2005$ ).

Viewed from this perspective, many of Brown/Levinson's strategies of positive and negative politeness need to be assigned to the category of non-polite/ appropriate rather than polite/appropriate behavior. This includes the use of terms of address, honorifics, indirect speech acts and many other forms of ritualized expressions, which are a fixed part of conversations and are thus routinely expected by participants. Since this canonical and institutionalized behavior does not constitute a conversational surplus, it should not count as explicitly polite either - even if that means breaking with traditional evaluations.

Despite their seemingly clear-cut distinction of types of relational work, the two authors do not leave unstated the fact that "there can be no objectively definable boundaries between these categories" (2005:12), and

[ $\mathrm{t}$ ]he points at which speakers perceive [appropriate] behavior to be 'polite' may, and certainly do, vary considerably from speaker to speaker, from community 
of practice to community of practice and even from one situational context to another in the case of individual speakers.

(Watts 2005: xliii)

This holds, of course, also true for the differentiation between other neighboring - or even distant - concepts within Locher/Watts' chart, such as the one between polite and over-polite behavior. Watts et al. (2005:8) elucidate this particular distinction with a rather unambiguous and hence convenient example of a request: While an utterance such as "Would you please close the window?" clearly minimizes a potential FTA, the utterance "Do you think I could possibly prevail upon you to close the window?" does not minimize the FTA any further, as one might expect, but, on the contrary, begins to have the opposite effect. In this case, less is clearly more, which is why "[o]ver-politeness is often perceived as negative exactly because it exceeds the boundary between appropriateness and inappropriateness" (Locher 2004:90). Although these two context-deprived utterances can serve as examples for polite vs. over-polite behavior respectively, this does not eliminate the possibility for the exact same utterances to be evaluated differently in another context by other participants. This means that predicting evaluations, even those of close friends, is virtually impossible since they are based on norms and standards, which have been accumulated individually in a lifelong process.

Then how come that communication - despite potential evaluative gaps between interlocutors' perceptions of interpersonal behavior - can still work out smoothly at all? Apparently, the intersection between interlocutors' evaluations, i.e. their common ground, must be big enough to avoid communicative breakdowns - at least in regular, everyday encounters, with exceptions surely proving the rule. Obviously, when judging the appropriateness of utterances, we seem to possess similar expectations, not only as receivers of (non)verbal behavior but also as their producers. In this regard, we can anticipate which reactions, i.e. evaluations, are expected from us (as receivers) and which reactions, i.e. evaluations, we can expect (as producers) (cf. Spencer-Oatey 2007). These two directions of the cognitive interplay between expectations and evaluations are depicted in Figure 14. Although this deductive process theoretically works for both producing and receiving participants, postmodern approaches stress the primacy of the hearer. In Figure 14, this is indicated through the diverging intensities of the two arrows, suggesting the preferred (right) reading path.

The similarity of our expectations, or rather the willingness to counterbalance diverging expectations, can be ascribed to interlocutors' basic human needs of association (see Section 4.3.1), which prompt them to behave in such a way that they are liked and considered as rightful members of their peer group. As a consequence, we can assume that communities of practice must possess certain norms for appropriate behavior, among them the code of conduct of TSR (see 


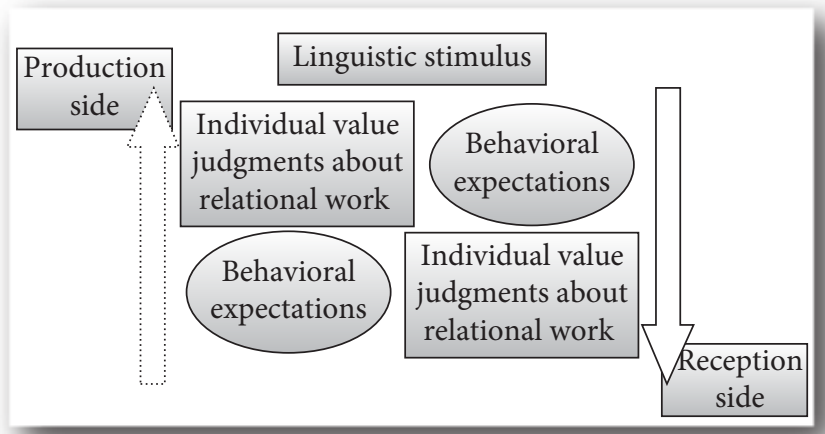

Figure 14. Cognitive processes of individual value judgments about relational work

Section 3.3.2), which members knowingly and willingly follow. This adherence to group specific norms, which mirrors the desire to conform to the expectations of a particular reference group, "engenders a structure and predictability which results in a considerable saving of energy in everyday life and can be viewed as serving the goal of something akin to social harmony and perhaps even survival" (Meier 1995:352).

As underlying frames of knowledge and social norms re-enter the scope of scientific interest concerning interpersonal relations, the social norm view (see Section 4.2) celebrates a comeback - at least to some extent. Werkhofer sees a need in coupling postmodern approaches with prepragmatic ones when he explains that

the modern view is biased towards a one-sided individualism [with] difficulties arising $[\ldots]$ from the corresponding neglect of social dimensions [which] tend to be overlooked or grossly underestimated by the adherents of this view. [A]ll versions of this view either neglect social realities completely or, adopting a remarkably simplistic, traditional approach, reduce them to only a small set of vaguely defined dimensions which are then relegated to a secondary status [...].

( $2005: 157)$

He concludes that while "neither of them [traditional and modern approach] can completely be rejected, neither of them is also fully acceptable" (ibid.). Thus, both perspectives should not be considered as rivaling alternatives but as complementing each other. To this end, Chapter 6 will fathom contextual factors, which may be influential for message board users' expectations and evaluations. 


\subsection{Impoliteness: The neglected stepbrother of politeness}

To completely cover Locher/Watts' framework, we still need to have a close look at the other half of the model of relational work, which encompasses types of negatively marked and thus inappropriate behavior, labeled over-politeness and impoliteness. Since over-polite behavior is usually regarded as "a less than optimal application of politeness patterns which in principle are perfectly acceptable in a given language or culture" (Kienpointner 1997:257), it is clearly of a different quality than impolite behavior per se. The latter needs to be dealt with in more detail, especially so since it is part of a regular terminological jumble which is used to designate various types of inappropriate behavior. While Culpeper (1996) uses the term impoliteness, we can also find rudeness (Kienpointner 1997), aggravating language (Lachenicht 1980), face attack acts (Austin 1990) and, in the field of $\mathrm{CMC}$, flaming, in scientific discourse.

For a long time, research in the field of impoliteness ${ }^{44}$ seemed to have been overshadowed by the omnipresent notion of politeness; in fact, it almost appeared to be treated as its neglected stepbrother (cf. Locher 2011). Although impoliteness is mentioned every now and then in most of the leading approaches to politeness, the focus was nevertheless on the more popular of the two siblings, politeness. Culpeper et al. summarize a common rationale to explain this lack of attention by stating that "an impoliteness framework is unnecessary, since Brown/ Levinson's (1987) politeness framework already postulates a category, bald on record, which accommodates 'impolite' phenomena" (2003:1547). But as there are what Culpeper et al. call "maximally offensive examples" (2003:1548) that fall outside the specific contexts of bald on record strategies, the idea that Brown/ Levinson's category could adequately encompass all kinds of impoliteness phenomena needs to be rejected.

This sustained lack of interest for impoliteness is also astonishing considering the fact that negatively marked behavior was always assumed to be easier to detect than behavior which conforms to politeness norms (cf. Mills 2002; Watts 2003). Still, merely a handful of approaches primarily dealing with impoliteness (and rudeness) is worth mentioning, a rather moderate number of publications compared to the vast amount of literature in politeness research. As impoliteness was hastily dismissed as a by-product of or a deviation from politeness, unworthy of being treated as an autonomous field of research, scientific approaches inadequately tried to explain impoliteness in terms of politeness, either by making use

44. Note that the notion of impoliteness is used here as a hyperonym for inappropriate behavior in general and thus includes various subtypes which will be dealt with in more detail in the course of this chapter. 
of models and methods which were originally designed for a completely different phenomenon or by deriving models for the description of impoliteness from politeness models. As Eelen points out,

the concepts involved can never explain impoliteness in the same way or to the same extent as they explain politeness. So the bias towards the analysis of politeness is not just a matter of differential attention, it goes far deeper than that: it is a conceptual, theoretical, structural matter. It is not so much quantitative, but rather a qualitative problem.

(2001: 104)

This way of proceeding can be exemplified with the help of three approaches, which take politeness, or, to be more precise, Brown/Levinson's model of politeness as a starting point for the classification of impoliteness:

\section{"Aggravating language - a study of abusive and insulting language"}

Lachenicht's (1980) approach ${ }^{45}$ investigates what he calls aggravating language, which he studies "as a rational attempt to hurt or damage the addressee" (1980:607) and which can be "performed with various degrees of intention to hurt" (1980:613). ${ }^{46}$ This hurt can be induced by two types of aggravation, which allude to Brown/Levinson's dichotomy of face in a rather obvious way:

Negative Aggravation: an aggravation strategy that is designed to impose on the addressee, to interfere with his freedom of action, and to attack his social position and the basis of his social action.

Positive Aggravation: an aggravation strategy that is designed to show the addressee that he is not approved of, is not esteemed, does not belong, and will not receive cooperation.

(1980:619)

The center piece of Lachenicht's paper is his modification of Brown/Levinson's theoretical system (see Section 4.3.1), to which he adds another branch, thereby extending it to abusive language (see Table 6). In line with Brown/Levinson, this adapted model takes FTAs as a starting point and assumes that they can be modified - the only difference being that modifications can lead not only to the weakening of their face-threatening potential (with mitigation, which corresponds to Brown/Levinson's category with redressive action) but also to their strengthening (with aggravation). The extra branch, containing positive and negative aggravation,

45. For a detailed critique of Lachenicht's model, dealing, among others, with the authenticity of his data and the selectivity between positive and negative aggravation, see Culpeper et al. (2003).

46. As will become obvious later in this chapter, Lachenicht's concept of aggravation equals with the notion of rudeness. 
Table 6. Lachenicht's strategies to modify FTAs (1980:621, my emphasis)

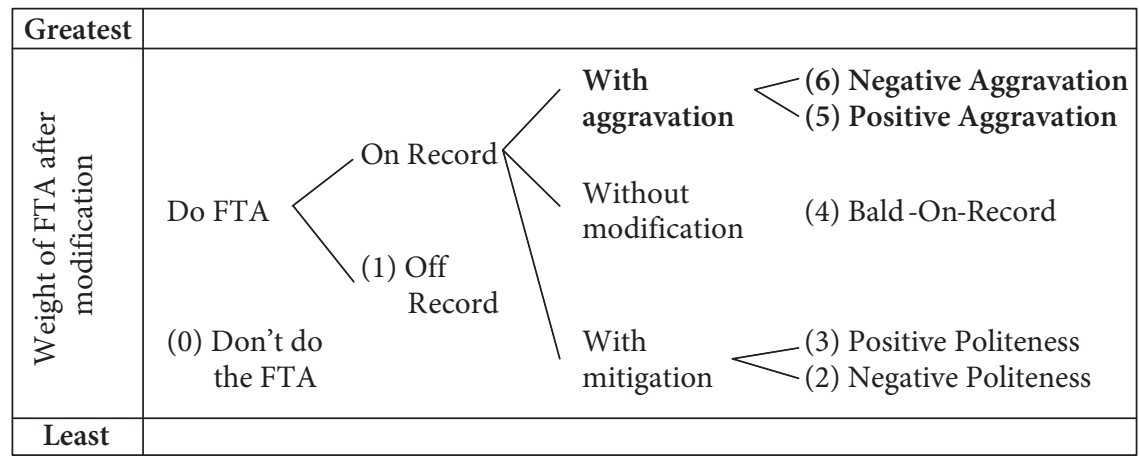

is added right above the familiar bald-on-record strategy to indicate the severity of the FTA's impact (mirrored also by the increasing numbers). Additionally, Lachenicht designates Brown/Levinson's bald-on-record and off-record strategies to serve as possible aggravation strategies.

To illustrate his own two concepts, positive and negative aggravation, he deliberately copies Brown/Levinson's course of action and provides an extensive compilation of linguistic strategies which can be used separately or in a combined way to aggravate face. Yet he hints that the "final realization of the superstrategy need not always be verbal [but] may consist of gifts, punches, reassuring smiles, raised eyebrows, gestures, and the tone of voice" (1980:622), thus indicating that "the range of techniques that may be employed far exceeds the bounds of linguistics" (1980:680).

\section{"Politeness revisited - the dark side"}

Another approach referring back to Brown/Levinson's taxonomy is presented by Austin (1990). Contrary to Lachenicht, she regards the construction of a model which attempts to impose a taxonomic structure as a futile endeavor (1990:291) still she relies on Brown/Levinson's superstrategies. Without questioning them, she claims that they are multi-functional in that "[f]ace attack is often, in fact, an application of the same principles which are used for face preservation; and many of the strategies for the one can be co-opted for the other" (1990:277). By face attack acts, Austin means "those communicative acts which are injurious to the hearer's positive or negative face, and are introduced in a situation which could have been avoided, but where their inclusion is perceived by the hearer to be intentional" (1990:279). As will be seen later on, face attack acts fit the description of rudeness.

For utterances to be interpreted "on the dark side", contexts were regarded as decisive - an insight which is the outstanding feature of her paper. Above that, she 
specifically stresses the role of hearers, thus concentrating her analyses more on their part - definitely a step in the right direction.

\section{"Towards an anatomy of impoliteness"}

A similar, yet more elaborate attempt to modify Brown/Levinson's classic model for the sake of inappropriate behavior is presented by Culpeper (1996). In order to draft "an anatomy of impoliteness", he does not extend Brown/Levinson's original model, but literally turns it upside down. He assumes that each of their politeness superstrategies has its opposite impoliteness superstrategy - meaning that impoliteness superstrategies are opposite in terms of orientation to face, which are thus means of attacking face instead of enhancing or supporting face (1996:356). What is more, Culpeper also adopts Brown and Levinson's formula for assessing the weightiness of an FTA: "The greater the imposition of the act, the more powerful and distant the other is, the more face-damaging the act is likely to be" (1996:357). Culpeper therefore invokes the following five strategies, which do not necessarily have to occur singularly but can be mixed:

1. Bald on record impoliteness: the FTA is performed in a direct, clear, unambiguous and concise way in circumstances where face is not irrelevant or minimised. It is important to distinguish this strategy from Brown and Levinson's Bald on record $[\ldots]$ where little face is at stake, and, more importantly, it is not the intention of the speaker to attack the face of the hearer.

2. Positive impoliteness: the use of strategies designed to damage the addressee's positive face wants.

3. Negative impoliteness: the use of strategies designed to damage the addressee's negative face wants.

4. Sarcasm or mock-politeness: the FTA is performed with the use of politeness strategies that are obviously insincere, and thus remain surface realisations.

5. Withhold politeness: the absence of politeness work where it would be expected (e.g. failing to thank somebody for a present may be taken as deliberate impoliteness).

(1996:356f., 2005: 41, my emphasis)

This clearly needs elaboration as obviously, some politeness strategies cannot be turned around to create impoliteness strategies that easily. While it works quite well with positive and negative politeness/impoliteness by employing a diametrically opposite set of (negative) instructions (e.g. "seek disagreement" or "invade the other's space"), the bald on record-strategy poses the first problem. This time, the strategy as such remains untouched, since - as some have argued before it already is an impoliteness strategy. Instead, and thus diverging from Brown/ Levinson's original model, the contextual conditions are turned upside down here, as there is a danger to face now. 
The fourth strategy resembles Brown/Levinson's off record-strategy insofar as the FTA is performed in a veiled, indirect way. But contrary to the original, Culpeper proposes the use of insincere politeness (and not rhetoric figures) to be deliberately hurtful (and not mitigate an FTA). As Culpeper's understanding of sarcasm strongly resembles Leech's view of irony, Culpeper (1996:356) refers back to Leech's definition of the Irony Principle (IP): "If you must cause offence, at least do so in a way which doesn't overtly conflict with the [Politeness Principle, see Section 4.2], but allows the hearer to arrive at the offensive point of your remark indirectly, by way of an implicature" (1983: 82). On the interplay of the IP and the PP, Leech (1983: 142) later expands as follows:

Apparently, then, the IP is $d y s$-functional: if the PP promotes a bias towards comity rather than conflict in social relations, the IP, by enabling us to bypass politeness, promotes the 'antisocial' use of language. We are ironic at someone's expense, scoring off others by politeness that is obviously insincere, as a substitute for impoliteness.

Consequently, politeness can be used in an inappropriate fashion. For this reason, the result of the IP is "the opposite of social harmony that is supposed to be promoted through Brown and Levinson's Off record politeness” (Culpeper 1996:357). Since irony is normally used for enjoyment and comity, Culpeper replaces Leech's notion of irony with sarcasm (or mock-politeness), which is supposed to cause social disharmony - a fact which is actually stated in Leech's definition of irony as well! ${ }^{47}$ Culpeper then goes on declaring sarcasm (and mock-politeness) as the opposite of banter (and mock-impoliteness, see 5.6), since the latter two usually cause social harmony (ibid.). With reference to Locher/Watts' integrative model, Culpeper's mock-politeness could therefore be regarded as a case of over-polite and thus inappropriate behavior, which is not used accidentally but strategically. Last but not least, withhold politeness, as the last of Culpeper's five strategies, seems to be the appropriate counterpart for the classic politeness strategy withhold the FTA.

Emulating Brown/Levinson's model even further - and consequently attracting the same points of criticism -, Culpeper (ibid.) also gives a provisional list of output strategies for positive and negative impoliteness. Accordingly, he issues a warning to remind his readership that this list is clearly not exhaustive, as strategies always depend upon an appropriate context to be impolite and can also be

47. The distinction between (harmonious) ironic and (disharmonious) sarcastic rudeness, which is based on the underlying intention of speakers rather than the surface form of potentially ironic or sarcastic utterances, is also shared by Kasper (1990:210f.) and Kienpointner (1997:263). 
conveyed by paralinguistic and non-verbal means such as the avoidance of eyecontact or shouting (Culpeper 1996:358).

As the three short overviews have proven, it is not necessarily easy to describe inappropriate behavior based on a model designed for politeness, as strategies cannot be adopted par for par. To my knowledge, attempts to do so have only taken recourse to Brown/Levinson's model, consequently inheriting a lot of its flaws and shortcomings. It is thus little surprising that such courses of action did not only result in descriptively inadequate comments on impoliteness, to say the least (Eelen 2001; Culpeper et al. 2003; Bousfield 2008), but above that, impoliteness also ran the risk of being discredited as "the parasite of politeness" (Culpeper 1996:355). Accordingly, and also true to the corresponding word formation process behind it, impoliteness was simply regarded as the polar opposite of politeness. An admittedly naïve equation based on an extremely oversimplified notion of politeness could therefore read as follows: impoliteness is attributed to the nonadherence or even the deliberate and conscious violation of socially sanctioned rights and obligations of politeness, like the lack of particles such as please, thank you, and sorry when expected by interlocutors in a particular situation. Back in 1973, Lakoff agreed with this premature point of view in arguing that impoliteness was simply the absence of politeness and a form of "plain speaking". Beside the fact that we do not know what exactly Lakoff considers to be "absence" of politeness, this assessment strongly contradicts with insights gained through Locher/ Watts' classification of relational work (see 5.3): A "lack of politeness" in whatever form does not automatically equal with impolite and inappropriate behavior, but might just as well be considered as non-polite and perfectly appropriate behavior. The polar perspective thus cannot be upheld.

Regarding politeness as the norm and rudeness as "merely pragmatic failure at achieving that norm" (Beebe 1995:156) needs to be discarded as a simplistic myth as well. A closer look into the matter easily proves that impoliteness is just as multi-faceted as politeness. A by all means perfect example to illustrate that point is presented by Beebe (1995: 161): In a busy New York restaurant, a group of people were repeatedly asked by different waiters if they would care to order. As they had made it plain that they intended to have a discussion over lunch and would therefore take their time over their meal, the attentiveness of the waiters "seemed to reflect a desire on the part of the waiters to get it over with, not a policy regarding length of stay, a lack of communication among waiters, or a problem with crowding, so it was viewed as rude". This scenario proves the point that "pushy politeness", as Beebe termed this special instance of over-politeness, is most probably evaluated as a case of negatively marked behavior. Beebe concludes that impoliteness is not automatically a failure to be polite, but that it can, 
on the contrary, also be a matter of tactic motivation and thus in fact "a reflection of pragmatic competence" (1995: 154) for the achievement of certain aims in a conversation. Impoliteness is thus non-cooperative but still (mostly) rational behavior.

\subsection{Distinguishing types of inappropriate behavior: Impoliteness vs. rudeness}

The remarkable terminological haziness between the two concepts of impoliteness and rudeness has often been tried to be explained away with respect to speakers' intentions. Terkourafi, for example, remarks that "[p]inning down the speaker's intention plays a decisive role for charting one's subsequent course of action [which is why] resolving the speaker's intention cannot be dispensed with" (2008:62). This entails, however, a severe problem, as vital importance is placed on yet another flighty and indeterminable factor for the assessment of the appropriateness of speakers' (non)verbal behavior. Terkourafi is very aware of the fact that there is no way of undoubtedly attaching these two labels to authentic conversations based on intentions, as they are not available for open inspection. Speaking with Culpeper et al., "[i]nterlocutors do not wear their intentions on their sleeves [and] what is in people's heads is accessible neither to analysts nor to interlocutors (nor even, ultimately, fully accessible to those whose behaviour is under investigation)" (2003: 1552, with reference to Grimshaw 1990:281). Observing analysts cannot count on participants' openly sharing their true intentions with others either because, as a matter of fact, candid statements about their intentions do not necessarily have to mirror the truth but can be deliberately deceiving.

Before, however, we start to use impoliteness and rudeness synonymously or choose one over the other in default of a reliable distinctive criterion, I intend to uphold Terkourafi's (2008:62) definition according to which impoliteness is regarded as accidental and sometimes attributed to speakers' ignorance or incompetence (as in cross-cultural communication), while rudeness is described as intentional. Rudeness is thus regarded as prototypically non-cooperative communicative behavior which usually destabilizes personal relationships between interactants. As examples of unintentional, hence accidental and unmotivated impoliteness, Kienpointner (1997:269) invokes the impolite behavior of children and foreigners, who just do not know better (yet). In both cases, we can witness the consequences of insufficient linguistic and/or cultural knowledge, which Thomas (1983) calls "pragmatic failure". But even adults within the same culture or language can produce impoliteness in the form of slips of the tongue or 
Freudian slips (Kienpointner 1997:269). In the following, I will give preference to the notion of impoliteness, reserving rudeness only for specific cases in which we have at least some kind of evidence that the speaker acted with bad intentions to willfully commit a face-threatening act.

Another way of approaching the matter is by examining the different functions of impoliteness which entails the appealing question whether it is inevitable to consider impoliteness as inappropriate behavior once and for all - especially in view of concepts such as Leech's (1983) mock-impoliteness or banter, which call into play a vital function of impoliteness in human communication. Following Kasper's definition, mock-impoliteness "extends to utterances which are overtly impolite yet blatantly false in their propositional content, and are thus understood as joking behavior" (1990:211). Obviously, we are dealing with a completely different function of impoliteness, one that is extremely marked, yet - in contrast to all the other functions mentioned before - in a positive rather than in a negative way. Strictly speaking, it is thus not even a kind of impoliteness - least not when we look at its function and not at its form - because it is not at all face-threatening but on the contrary face-maintaining or even face-enhancing. For this reason, superficially impolite utterances cannot automatically be judged as inappropriate once and for all, which is why I propose a differentiation between two functions of impoliteness, which are based on the (intended or unintended) effect a speaker's utterance has on his interlocutor's face (see Table 7).

The advantage of this model is its hearer-orientation. We are not reliant on non-tangible speakers' intentions of whatever nature, but can base our judgments on parameters which are comparatively easy to access: hearers' reactions, which mirror their evaluations of the appropriateness of utterances at least to some degree. As discussed before, the differentiation between the notion of impoliteness or rudeness within the left column remains problematic, as it strongly depends on

Table 7. Types of impoliteness according to face-orientation

\begin{tabular}{ll}
\hline \multicolumn{2}{c}{ Types of impoliteness according to face-orientation } \\
\hline face-threatening & face-maintaining / face-enhancing \\
negatively marked impoliteness: & (positively) marked "impoliteness": ${ }^{8}$ \\
- impoliteness & - mock-impoliteness \\
- rudeness & - banter \\
$=$ inappropriate & $=$ appropriate \\
\hline
\end{tabular}

48. Strictly speaking, the term impoliteness can only be used for a superficial, formal description. From a functional perspective, however, we are clearly not dealing with impoliteness at all. 
Table 8. Modified model of relational work

\begin{tabular}{|c|c|c|c|c|}
\hline \multicolumn{5}{|c|}{ Relational work } \\
\hline $\begin{array}{l}\text { negatively } \\
\text { marked }\end{array}$ & $\begin{array}{l}\text { (positively) } \\
\text { marked }\end{array}$ & unmarked & $\begin{array}{l}\text { positively } \\
\text { marked }\end{array}$ & $\begin{array}{l}\text { negatively } \\
\text { marked }\end{array}$ \\
\hline $\begin{array}{l}\text { impolite } \\
\text { rude }\end{array}$ & $\begin{array}{c}\text { mock-impolite } \\
\text { banter }\end{array}$ & non-polite & polite & $\begin{array}{l}\text { over-polite } \\
\text { mock-polite }\end{array}$ \\
\hline inappropriate & appropriate & appropriate & appropriate & inappropriate \\
\hline
\end{tabular}

the state of evidence for speakers' negative intentions. With these two functions of impoliteness separated, we can now add a new column to Locher/Watts' (2005) framework of relational work, representing (positively) marked "impolite" behavior as displayed in Table 8. This new column enriches the original model with one more variety of appropriate behavior - only this time in combination with formally "impolite" behavior. As this type of formal impoliteness is extremely marked, rather positively than negatively, the next chapter will reveal the working principles behind both types of "appropriate impoliteness".

\subsection{Appropriate impoliteness: Mock-impoliteness and banter}

Although swearwords and "other linguistic phenomena which are assumed to be typical examples of rudeness ${ }^{49}$ in many languages/cultures, for instance loudness of voice (shouting), frequent interruptions, bare imperatives, taboo words, ironic remarks, aggressive jokes, indirect attacks, etc." (Kienpointner 1997:255) are strongly biased towards an impolite interpretation - at least from a purely formal point of view -, they might as well be a sign of something completely different: familiarity, solidarity or even a signal of intimacy (cf. Lachenicht 1980:608; Leech 1983: 144; Terkourafi 2008: 68). In this case, mock-impoliteness and banter are instances of a special type of "impoliteness that remains on the surface, since it is understood that it is not intended to cause offence" (Culpeper 1996:352). As established before, a prototypical feature of impoliteness, non-cooperativeness (cf. Kienpointner 1997:258), does not hold for these two kinds of appropriate impoliteness. A closer look at the characteristic workings of these two phenomena

49. For the sake of legibility, one needs to keep in mind that Kienpointner (1997) prefers the term rudeness over impoliteness. The use of the term rudeness thus only mirrors Kienpointner's wording. It is not, however, used intentionally to imply the difference between the two notions as established above. 
might help us tell them apart. In order to do so, let us start with Leech, who introduced the banter principle:

In order to show solidarity with $h$, say something which is (i) obviously untrue, and (ii) obviously impolite to $h$ [which leads to the interpretation that] what $s$ says is impolite to $h$ and is clearly untrue. Therefore what $s$ really means is polite to $h$ and true.

(1983: 144)

Whether utterances as those described by Leech are automatically polite just because they are not impolite has been questioned before. It should go without saying that mock-impoliteness as well as banter can only be assessed correctly and thus appreciated if interlocutors know each other well. Otherwise, utterances such as "You silly bugger" (Kienpointner 1997:261) could easily be misinterpreted as truly face-threatening, when used between relative strangers. Kienpointner interposes that although mock-impoliteness can be a bit risky in rather formal circumstances, it can nevertheless be successfully used to relax the stiff atmosphere (1997:262). The question whether interlocutors who are in fact close to each other still sense the slightest face-threat within mock-impoliteness or banter can only be answered individually by the interlocutors involved. I argue that if mock-impoliteness and banter are appropriate in a given context, chances are rather slim that a face-threat is actually perceived. On the contrary, odds are that a face-maintaining, if not even a face-enhancing stance is detected by the hearer; according to Leech, banter reflects and fosters social intimacy (1983:144). He also points out that in intimate relationships, the necessity and importance to show politeness tends to decrease (ibid.). Consequently, not showing explicit means of politeness and even being superficially impolite can paradoxically be associated with and promote intimacy (cf. Culpeper 1996:352).

In fact, Wolfson (1988:32) came to the same conclusion: In closely examining social distance, Wolfson's bulge model of interaction shows that "speech behavior tends to be most frequent and most elaborated between those who are acquaintances and casual friends, rather than between intimates or strangers" (Holmes 1995: 13). With intimates and strangers marking the two extreme poles on a scale of minimal and maximal social distance respectively, Wolfson perceives a bulge-shaped curve spanning between these two extremes: While behavior among intimates and strangers seems to show great similarities in terms of reduced (interpersonal) attention, there is a marked difference in the more elaborate interpersonal behavior of acquaintances and casual friends at the center of the bulge. Wolfson explains her findings with the relative degree of stability of the relationships involved. 
Although mock-impoliteness and banter are often used synonymously, the latter one stands out as being more ritualized in form, which is why Kienpointner uses the term ritual insults ${ }^{50}$ in his taxonomy, defining them as "sophisticated systems of syntactic and semantic rules [...], known in different languages and cultures all over the world, especially among young male adults" (Kienpointner 1997:262f.). As a kind of language game, known in America as sounding, playing the dozens or signifying, it usually takes place particularly amongst black adolescents (cf. Labov 1972; Culpeper 1996:353). On the basis of shared knowledge within a peer group (see Section 6.3), the key to understanding ritualized banter lies in taking insults in a non-serious way and perceiving them as obviously untrue. Only then can banter be considered as a game: purely competitive, totally detached from actual facework, with winners (those who have the widest range of insults at hand) and losers. Ritual insults thus do not endanger social relationships but are often used as "a societal safety valve" (Culpeper 1996:353), which enhances group solidarity. Unlike real insults, "the more exaggerated ritual insults are, the less they are in danger of being taken seriously" (Kienpointner 1997:263). Typical cases of banter are introduced by Labov in his renowned study Language in the Inner City: Studies in the Black English Vernacular (1972). The target of these insults usually being someone's mother, those forms of banter also go by the name Yo $\operatorname{Mam}(m)$ a Jokes:

J1: Your mother take a swim in the gutter.

J2: Your mother live in a garbage can.

J1: Least I don't live in 1122 Boogie Woogie Avenue, two garbage cans to the right.

Labov (1972) warns that it is consequently the "weak" insults which may cause the most trouble, as this kind of behavior may be interpreted as real personal insults and thus as truly inappropriate behavior (cf. Culpeper 1996:353). In equal relationships, impoliteness has a tendency to escalate due to a lack of "a default mechanism by which one participant achieves the upper hand" (1996:355). An insult can thereby trigger a counter-insult which may set in motion a spiral of insults, possibly even including physical violence. This leads us to the dynamics of insults and corresponding counter-measures as described by the notion of flaming.

50. Ritual insults can also be witnessed in army recruit training discourse, where they appear to be the norm rather than the exception. Drill sergeants' behaviors may or may not be evaluated by recruits as impolite, as the face-threat is based on "ritualized and institutionalized codes of linguistic behavior" (Mills 2005:270). 


\subsection{Strings of inappropriate online behavior: Flaming}

[L]ay observers commonly represent flaming as a highly negative message that functions like a metaphorical flamethrower that the sender uses to roast the receiver verbally.

(O’Sullivan/Flanagin 2003:68f.)

The presumed reasons for the occurrence of flaming - among them anonymity, text-based communication without social context cues, an initial lack of a normative code of conduct, cultural diversity and disparate interests, needs and expectations - have already been outlined in Section 2.6.1. In the past, this term has been used abundantly to label the most diverse phenomena: swearing, insults and name-calling, but also an increased willingness to communicate bad news or negative information or even expressions of personal, emotional feelings towards other people. It is therefore imperative to delineate the notion of flaming as precisely as possible. ${ }^{51}$

Originally borrowed from computer subculture, to flame meant "to speak incessantly and/or rabidly on some relatively uninteresting subject or with the patently ridiculous attitude" (Steele 1983:63) - not only in CMC but in general. Flaming in this sense was thus equaled with violations of Grice's maxims. In recent years, however, semantic changes of this notion seem to have limited its scope of application to forms of computer-mediated communication only. According to general consensus, flaming is "hostile and aggressive interaction" (Thurlow et al. 2004: 70) directed towards an immediate interlocutor. Accordingly, my reading of flaming is restricted to ad hominem attacks only. Whether repetitious punctuation, constant capitalization, swearing and other expressive and uninhibited verbal means count as flaming, needs to be decided by interlocutors, making flaming another emergent and evaluative notion. Being a co-constructed phenomenon very similar to other types of inappropriate behavior, we cannot speak of flaming if nobody seems to take offence (cf. Avgerinakou 2003:276), which is why we are again reliant on interlocutors' (explicit) reactions. What is more, flaming in tightknit groups is not automatically negative and destructive but can, on the contrary, be used just like mock-impoliteness or banter to express identification with the group and convey a sense of belonging and camaraderie. Since "affiliation reduces the danger of threats to interpersonal face" (Arndt/Janney 22005:39), the term pseudo-flaming or even mock-flaming clearly is in order in these cases.

51. For an ample collection of definitional approaches to the notion of flaming see Turnage (2007). 
If the notion of flaming encompasses the same interpersonal functions as impoliteness, or even rudeness, and mock-impoliteness - only in an online context - how come we need this additional notion at all? As a matter of fact, there is only one characteristic that does set flaming apart from all the other notions, prompting some users to even speak of flame wars for dramatic effects: Flames can only be witnessed in strings of utterances, the minimum being two causally but not necessarily spatially consecutive inappropriate entries, in which one impolite utterance is followed by another and maybe even by yet another. In other words, flames involve one, sometimes more users in reciprocally exchanging ad hominem attacks, which can be interspersed with unmarked, pacifying comments of third party interlocutors. Thus describing a structural quality rather than a functional one, the notion of flaming does not qualify to be incorporated into Locher/Watts' (2005) framework of relational work. It could, however, be seen as a structural hyperonym to all the other types of inappropriate behavior - at least for the sphere of online interaction.

The notion of flame war is insofar more fortunate as it reflects the dialogic or even polylogic quality of a process, which continues until one of the parties involved gets bored and is too tired to continue. To illustrate this process, Shea cites a USENET (see Section 2.4) participant who describes the typical sequence of a flame war and points out that, at least in his view, they are ubiquitous:

${ }^{*}$ Every ${ }^{*}$ discussion list of which I have been a part - no matter what its subject has fallen victim to such ills - a few have gone down in e-flames. The pattern is absolutely consistent. Writer A drops a light remark - always ${ }^{*}$ tangential ${ }^{\star}$ to the main discussion. Writer $B$ interprets the message in the worst possible light and fires off an outraged reply, in which writer A is called a racist, a classist, a fascist - whatever seems to apply. Writers C-L chime in, rather like the crowds in a DeMille film, muttering 'Shame!' or 'I agree!' or 'A is right!' or 'B is right!' Writer A replies saying, 'Gosh, it was just a joke. I'm not a fascist. Lighten up. Writer B says, 'This issue (the South, date rape, Nicaragua) is DEADLY SERIOUS. I won't lighten up. I won't.' By the time things have cooled down, Writers A and B have left the list; or Writers N-DD have left the list; or the list has died. These are not ${ }^{*}$ odd $^{*}$ occasions - they happen to *every list.*

Investigating ways of resolving attacks in a USENET group, Baker (2001) lists and explains the following, CMC-specific strategies:

1. Advocate "sympathy/understanding" (albeit issued with a patronizing and superior tone, one of the least aggressive resolution strategies);

2. Laissez-faire (ignore the attacker);

3. Flame (by far the most common resolution strategy was to respond in kind); 
4. Threat of physical violence (less common, more aggressive and probably facetious means of challenging);

5. Censorship (complaining to the postmaster in charge to express intention of denying the attacker e-mail access, in effect to censor him);

6. Exposure (outing a user by listing all of his postings to a Usenet group, about any subject, thus substantiating any antagonistic tendencies).

In fact, flames hardly ever arrive in an agreement between the opponents. Third party interventions, on the other hand, are all the more common and involve other users in attempting to reconcile or least to silence the flaming parties. In this regard, the fifth strategy, the contacting of the attacker's system administrator, can be seen as the last and certainly most extreme resort in online environments (Lee 2005:388).

\subsection{Summary: Implications of an integrative perspective for the analysis}

In the course of finding a working definition for politeness from a postmodern, discursive point of view, a strategy for the handling of the slippery notion of politeness in the forthcoming empirical analysis emerged. It became obvious that labeling utterances as polite always involves a certain amount of subjectivity on the part of the observing analyst. This bias can, however, be reduced by allocating the hearer perspective, i.e. fellow users involved in a string of message board conversation, and contextual variables a central position within the analytical framework.

Above that, the notion of politeness was put in perspective and integrated in Locher/Watts' framework of relational work. With the advent of appropriate behavior, the scope of politeness was reduced considerably, leaving only those utterances to the label polite which feature a surplus of consideration towards interlocutors. This revised notion of politeness was then contrasted with inappropriate behavior. The vagueness between the two terms impoliteness and rudeness could not, however, be eliminated completely since the only difference lies in a willfully hurtful intention on part of the speaker in the case of rudeness, which can, of course, never be proven without doubt in actual data.

A special case of impoliteness could be found in mock-impoliteness (and its ritualized version banter): Utterances which appear impolite and hurtful on the surface level actually aim at creating solidarity and consequently usually lack any face-threatening force. In the right context, mock-impoliteness as (positively) marked behavior is thus absolutely appropriate. As a distinct type of relational work, it was assigned a place of its own in Locher/Watts' model, which was 
expanded accordingly to serve as a profound basis for the upcoming empirical analysis.

The last of the subtypes of impoliteness to be distinguished, flaming, was not granted that privilege, since it cannot be set apart from types of impoliteness functionally. It can, however, be considered a hyperonym of negatively marked behavior of whatever sort, as it designates a particular structural feature that can theoretically be sported by all of them: strings of utterances which are used to reciprocate inappropriate behavior over a longer stretch of time. 



\section{CHAPTER 6}

\section{Prelude to the analysis}

\section{Gathering contextual factors}

\subsection{Introduction}

[C]ontext can be the whole world in relation to an utterance.

(Pinkal 1985:36)

As already hinted in previous chapters, it is imperative to base "objective" analy$\operatorname{ses}^{52}$ of interlocutors' interpersonal relations and their very own assessments thereof on two major keystones: speakers' and hearers' (non)verbal actions and reactions as well as contextual factors, which might have influenced these kinds of (non)verbal choices in the first place. The relation between these two cornerstones is depicted by Goodwin/Duranti (1992:3) through the simple binary distinction between figure and ground, as the notion of context involves "a fundamental juxtaposition of two entities: (1) a focal event; and (2) a field of action within which that event is embedded." Although contextual factors certainly help solidify judgments which would otherwise be based on hearers' (non)verbal reactions alone,

$[\ldots]$ it is often difficult to put one's finger on exactly what aspects of the situation ought to count as context and sometimes even more difficult for the analyst to gain access to those aspects of the communication, especially when they involve the 'private' actions of people sitting alone in front of their computer screens.

(Jones, R. 2002: 21)

Observing outsiders can therefore barely guess which of these factors actually triggered a certain assessment and to which degree. As this will remain the hearer's secret - just like the intentions behind a speaker's utterance - we always run the risk of over- or underestimating certain variables. What is worse, we might not even have access to some or be aware of other variables.

The intriguing notion of context has been in the center of pragmatic attention since the discipline's inauguration and has ever since been used as a pretext to

52. Despite arduous efforts to consult all kinds of sources for an analysis that is as objective as can be, we are always left with a subjective tinge of the analyst's very own perspective. According to Ehlich, the object of study "can only be researched from within, not from some seemingly external position" (22005:74). 
explain away unexpected results of analyses. But what exactly does context stand for? Taking Schmid's proposal as a first landmark, context is "anything that can have influence on the interpretation of an utterance" (2002:435). As convenient and appealing as this description may seem, this broad definition certainly needs refinement. First and foremost, context is never a fixed and solid entity which is simply "out there" for us to find. We should therefore stick to those approaches which point out that context is, in fact, a cognitive construct, which is just as emergent and dynamic a notion as is politeness, because in speaking and understanding, speakers and hearers create current contexts for current utterances (cf. Goodwin/Duranti 1992; Gumperz 1992; Quasthoff 1994; Bazzanella 2002; Bublitz 2003 and Clark 2006). This can easily be illustrated considering the fact that the moment a thought is uttered, it instantaneously becomes part of the context and thus relevant for the interpretation of the following as well as the preceding utterances. What is more, contextual factors, such as power relations, do not stand in a simple unidirectional cause and effect relationship with politeness and related phenomena. On the contrary, the dialectic relationship between the two leads to a transformation of both interpersonal relations and contextual factors within communicative exchanges (cf. Kasper 1990:203). To a greater or lesser extent, contexts are thus constantly changing. Consequently, Schmid fittingly remarks that "context resides neither in the surrounding text nor in the situation, but in the mind" (2002:442). For this reason, context is "never completely shared by participants" (Auer 1995: 13). This is especially true for CMC in general and message board interaction in particular, where users usually participate from separate places and time (cf. Chapter 2).

There are, however, some influential variables which are not liable to change and which can thus indeed be shared completely by participants. Bublitz regards these factors as "the given infrastructure of an utterance" (2003:382, original emphasis), which he calls environment, thereby stressing its rather stable nature in contrast to the fluctuating character of context. Of course, not all of these "resources" (cf. Mercer 2000:44) within the environment are automatically relevant for the ongoing interpretative process since only those which have in fact significance for the negotiation of meaning are turned into context. Following Bublitz, "context is interpreted environment" (2003:383).

As a prelude to the analysis, this chapter tries to bring together those influencing variables from the online and the offline world which presumably have an impact on the interpretation of interpersonal relations in online message boards (cf. Savas 2011 for chats). May the determination of relevant contextual factors be a diligent task for FtF interaction, it is even more so for CMC, where possibilities to monitor our interlocutors' faceted social presence in multiple social situations 
Table 9. Three dimensions of context

\section{Dimensions}

- personal (context)

- interpersonal (context)

- (extra-) medial (environment)

- location

- accessibility

- verifiability

are clearly limited. The framework for the investigation of contextual factors in message boards used in this chapter is based on earlier approaches such as Firth (1957), Hymes (1974, 1986), Murray (1988), Herring (2007) etc. Although the eight components of Hymes' model for the ethnography of speaking (setting/scene, participants, ends, act sequence, key, instrumentalities, norms, and genre) will partly resurface within the three major categories proposed here, the tripartite structure itself is reminiscent of Murray's proposal to distinguish between three dimensions: the participant, the activity being engaged in (the interpersonal axis), and the physical dimension or setting (including both the physical environment in which communication takes place as well as the various channels available for communication). In maintaining Bublitz' differentiation between (emergent) context and (static) environment and by ascribing message board specific location, accessibility and verifiability to these dimensions, the following analytical grid presents itself (see Table 9).

Emphasizing once more the interrelations between the online and the offline sphere, users automatically draw on their FtF experiences and expectations - even or especially in the context of context. For that reason, online interaction cannot be divorced from offline contexts and a good deal of shared understanding among participants (even among newbies) is directly imported from the offline world. Although most of these factors can easily be compared to those regularly occurring in $\mathrm{FtF}$ interaction, some of them may reappear as a medium specific equivalent in message boards.

\subsection{The participant: Personal contextual factors}

The first dimension is solely dedicated to the participant, dealing with all those factors of a user's personal background which can but do not necessarily have to be turned into context for the evaluation of (interpersonal) utterances:

- appearance: voice, bodily features, clothes etc.;

- demographics: gender, age, ethnicity, social class, educational level, occupation, status, region etc.; 
- current feelings, moods etc.;

- cultural background;

- knowledge gathered in past experiences.

Although neatly ordered in a list, which makes no claim of being exhaustive, it is the interplay of all these (and coming) factors within a user's mind, which turns their demarcation as influencing variables into a guessing game. While most of the factors are quite straightforward, others are in themselves indistinct, which is why at least for the last two bullet points, some elucidations are in order.

Culture is insofar an important influencing variable as it sets the course for participants' very particular face sensitivities, ${ }^{53}$ strategies in relational work, as well as conceptions of (role-related) rights and obligations (Spencer-Oatey 2002:543) (cf. social norm view, Section 4.2). Naturally, "people from different cultures often have not only different ideas about what counts as language, but also different ideas about what count as imposing, options, friendliness, dominance, supportiveness, and other key concepts" (Arndt/Janney 22005: 29f.). Accordingly, users' expectations and interpretation of their own as well as their interlocutors' behavior are closely linked to their cultural background. Culture is also a dynamic notion, which can be defined as "traditional (i.e. historically derived and selected) ideas and especially their attached values" (Clyne 1994:2). In focusing on its inner make-up, Spencer-Oatey adds that culture is "a fuzzy set of attitudes, beliefs, behavioural conventions, and basic assumptions and values that are shaped by a group of people" (2000a:4). In structuring these components, she regards basic assumptions and values as belonging to the inner core, while attitudes, beliefs and social conventions are part of the outer core, resulting in (non-)verbal surfacelevel behavioral manifestations (ibid.), which are hence only the condensate of the underlying cultural framework and need to be evaluated as such.

These are, of course, only two of numerous ways to define this notoriously difficult term. As Watts points out, its use "ranges from national groupings, through languages, gender-specific differences, social classes, subcultures determined by interest groups, age groups, in-groups, etc. and back to broad, sweeping notions such as 'western European and North American culture', 'Asian culture"' (2003: 101). For the purpose at hand, I will stick to the above definitions, keeping in mind that even with a clear-cut, comprehensive definition of culture at hand, there is no way for us to access users' real life cultural influences, as they are simultaneously members of different cultural groups anyway. Even more so in CMC environments, where we can only keep our eyes open for implicit clues within

53. For a comprehensive and supposedly universal framework of ten value constructs, which form the basis of individual face sensitivities, see Schwartz (1992) and Schwartz et al. (2001). 
users' messages: As studies by Johnstone/Bean (1997:222f.) have proven, speakers deliberately choose how they sound, meaning that conscious speech choices are used as an instrument to identify with larger groups or individuals. These choices concern all levels of language (among them dialect stereotypes, unconventional figurative language, poetic repetition, alliterations etc.) and can serve as devices for the creation of a personal style and thus a social self.

Although users' points of views are clearly biased by their cultural background, they are not submissive puppets on a string. On the contrary, their very own experiences as individuals within cultures must be ascribed equal importance, which brings us to the last item on the list: knowledge gathered in past experiences. Obviously interwoven with the notion of culture, a special form of shared knowledge, it encompasses not only interactional patterns, proficiencies and skills with regard to language but also knowledge on how to handle a computer, what to do in CMC and how to communicate via TSR. These random examples of past experiences can be grouped into types of knowledge, the first basic distinction being the one between procedural knowledge ('knowing how to') and factual or declarative knowledge ('knowing that' or 'knowing about') (Rumelhart/Norman 1981).

The latter notion comprises three further subtypes: Linguistic knowledge describes the knowledge of the general principles and strategies of human verbal interaction. Tulving (1972:386) compares it to "a mental thesaurus, organized knowledge a person possesses about words and other verbal symbols, their meaning and referents, about relations among them, and about rules, formulas, and algorithms for the manipulation of these symbols, concepts, and relations". With a view to this type of knowledge, we have to remember that, in theory, message boards are open for everyone all over the world, not only native speakers of English. Linguistic knowledge, just like any other type of knowledge, is thus totally user-specific and can differ immensely among users.

Another important component of factual knowledge is episodic knowledge (also known as episodic memory) which "is concerned with unique, concrete, personal experience dated in the rememberer's past" (Tulving 1983:v) - as, in the context of message board conversations, previous exchanges with other users. In Tannen's words, "[i]n a series of conversations between the same people, each encounter bears the burdens as well as the fruits of earlier ones" (1992:23). In message boards, these burdens and fruits are put down in writing, as these encounters are automatically stored in archives. One could therefore say that (parts of) a user's message board related episodic knowledge is preserved and portrayed electronically - not unlike a diary open to public - to be accessed by anyone, any time (see Section 2.4). Due to these message board structures and in contrast to FtF communication, it is extremely easy for rather personal episodic knowledge to "go public", first and foremost by quoting older posts from the archive, thus 
transforming them from comparatively private to public knowledge, ready to be shared by the entire CoP.

This transformation is most natural for the third and last type of factual knowledge: Semantic or conceptual knowledge is easily turned into shared knowledge since it "refers to a person's abstract, timeless knowledge of the world that he shares with others" (Tulving 1983:v). Conceptional knowledge, like being familiar with the concept of a computer or a message board, is insofar impersonal, as it is detached from and independent of personally experienced episodic memory dated in the past.

As has become obvious in the last paragraph, it is imperative for users to share at least part of their knowledge with interlocutors in their $\mathrm{CoP}$ in order to secure smooth and trouble-free conversation. This is all the more endangered the less overlap between users' mutual knowledge or common ground (Clark 1982, 2006) there is, including expectations for appropriate behavior. In a nutshell,

the more convergent people's situational assumptions are in a given instance, the more they feel that they understand each other; and the more divergent their situational assumptions are, the less they feel that they understand each other. Conflicts arise when people repeatedly fail to achieve some type of convergence.

(Arndt/Janney ${ }^{2} 2005: 32$ )

Looking back at the introductory list, it is fairly obvious that most of its factors are rooted in the offline world. Therefore, it is interesting to see if and how they can be transferred online. Table 10 gives an overview of TSR templates and other structural features of this particular message board (see Section 2.6.2) which offer users a platform for self-portrayal, also including explicit self-definitions within the verbalized contents of posts, which are typical for message board newbies.

Obviously, users and their individual traits are in fact visible in each and every post they submit: As Table 10 shows, most of the user related information is presented in the form of pre-filled templates, which are inserted automatically in each post. Above that, peripheral elements outside of the post, which accompany contributions but are not an integral part thereof, can further substantiate (but also contradict) first impressions. Finally, users have the opportunity to round off their self-portrayal independent of templates and in various ways by manipulating and playing with the contents of their posts as such. What is interesting is the fact that users are virtually "present" even if they are not participating synchronously: By means of older posts and said central and peripheral elements, their self-presentation is available even if the person is not. In this sense, users always inhabit both offline and online environments simultaneously, which led some researchers to consider the self as multiple (Stone 1995; Turkle 1995). R. Jones speaks of "a 
Table 10. Message board structures for self-portrayal

\begin{tabular}{|c|c|c|}
\hline & $\begin{array}{l}\text { Central elements } \\
\text { (within the post) }\end{array}$ & $\begin{array}{l}\text { Peripheral elements } \\
\text { (outside of the posts) }\end{array}$ \\
\hline $\begin{array}{l}\text { Template dependent } \\
\text { inserted automatically }\end{array}$ & $\begin{array}{l}\text { - nickname } \\
\text { - avatar } \\
\text { - labels } \\
\text { - statistics } \\
\text { - signature } \\
\text { - reputation system } \\
\text { - societies }\end{array}$ & $\begin{array}{l}\text { - profile information } \\
\text { - links to personal } \\
\text { webpages, weblogs, } \\
\text { facebook accounts and } \\
\text { other social network } \\
\text { sites }\end{array}$ \\
\hline $\begin{array}{l}\text { Template independent } \\
\text { inserted individually }\end{array}$ & $\begin{array}{l}\text { - embedded objects (real life pictures, } \\
\text { sound files, videos, etc.) } \\
\text { - explicit self-definitions } \\
\text { - idiosyncratic formal make-up of } \\
\text { posts (fonts, sizes, colors, emoticons, } \\
\text { capitalizations, emotes, etc.) }\end{array}$ & \\
\hline
\end{tabular}

new kind of interactional accessibility involving new ways of being present and monitoring others' presence" (2002: 17, original emphasis).

Still, a lot of details that make an individual unique cannot be expressed through templates or other message board specific compensatory mechanisms to mimic FtF or real life conditions. This holds especially true for those features which cannot be captured in numbers or pictures but which have to be distilled from whatever can be read between the lines in messages, such as a person's cultural background or educational level, to name but two. Regarding the search for users' background information, Herring points out that a lot of information "is given off largely unconsciously by [users'] sophistication of language use, including adherence to prescriptive norms" (2001:621), thus possibly revealing more authentic information than any strategically filled template ever could. While deliberately stated information indicates how one wishes to be perceived, there are also much more subtle, even unintentional clues slipping almost unnoticed as, for example, contained in writing style, proper use of abbreviations and the like within messages. Although both forms of expressions can be used strategically, the second one is certainly much harder to control (cf. Donath 1999:38).

In any event, we cannot assume that all personal factors, whether given consciously or not, are carried over truthfully, as "the presentation of self is tilted away from the physical axis [which] paves the way for the manipulated presentation of alternative, ephemeral, and disposable selves through our relationships with others" (Lea/Spears 1995:220). Theoretically, this means that the technical make-up of message boards leave a lot of leeway for their users to creatively reinterpret 
Table 11. Users' options for self-portrayal online

\begin{tabular}{llll}
\hline Mirroring self $\rightarrow$ & Masquerading self $\rightarrow$ & Borrowing self $\rightarrow$ & Fabricating self \\
\hline $\begin{array}{l}\text { real }: \text { virtual }=1: 1 \\
\text { "'WYSIWYG") }\end{array}$ & $\begin{array}{l}\text { slight alterations, } \\
\text { foregrounding or } \\
\text { downplaying traits }\end{array}$ & $\begin{array}{l}\text { pretending to be } \\
\text { someone else }\end{array}$ & 100\% fictitious \\
\hline++ & \multicolumn{1}{c}{ degree of authenticity } & -- \\
\hline
\end{tabular}

and manipulate their self-portrayal online. This can be achieved either by leaving out or de-emphasizing certain individual traits or by foregrounding, altering or plainly inventing others. Table 11 depicts a scalar chart of users' options for selfportrayal with two extreme poles and a gradual decrease of authenticity.

In order for us to verify users' self-presentations, we would need access to their real life data to make the comparison. But there lies the rub: With the exception of those rare cases in which we know our interlocutors from previous FtF encounters, there is no way for us to access "the real person" behind the keyboard. Since even personal information made explicit in users' posts or portrayed on users' personal webpages, weblogs, facebook-accounts and the like can be a hoax, we have to settle for dealing with online personae of varying degrees of truthfulness. Hints to a user's potential insincerity and attempt to deceive could, however, be found in the rare but nevertheless possible cases of contradicting information given about an online persona. In any case, the consequence of not really knowing about interlocutors - be it from an inside (user) or from an outside (observer) perspective - remains the same: Users' perceptions of interactions and our assessments thereof can exhibit discrepancies. Despite or even because of the fact that our analyses are based on message contents and on possibly doctored contextual factors, our input could, in the worst case, be contradictory.

In this connection, several questions have been posed repeatedly: How big are the differences between online personae and their real life originators, sitting in flesh and blood behind their keyboards? In other word: How much identity construction is really going on in message boards? Is it as bad as it appears to be? Is the term identity construction even justified or should we talk about face constitution instead? Especially with regard to our key concepts, relational work and facework, we have to ask ourselves how face is actually constituted in this computer-mediated environment. 


\subsubsection{Face constitution in online message boards}

[W] can be multiple, a different person... each time we enter cyberspace, playing with our identities, taking ourselves apart and rebuilding ourselves in endless new configurations.

(Bell 2000:3)

The first step in answering all these questions and in validating the substance of the above quote certainly lies in a distinction between identity and face, two terms which should be kept apart carefully (cf. Cupach/Metts 1994; Ting-Toomey 1988; Tracy 1990). While face is the epitome of social self is a dynamic, emergent notion, which is constantly negotiated in interaction (see Section 4.3), identity is an individual, much broader phenomenon (Arundale 2006:202). Admittedly, both notions have a lot in common: They are both interrelated and multi-faceted as they are composed of multiple attributes and arise, are maintained and changed with the help of others in dialectic processes - each to a certain extent. For this reason, Giddens (1991) refers to identity as "projects of the self" which are under current, life-long construction. We should not make the mistake of considering identity as something unitary, fixed and stable per se, which has matured completely by the time we reach adulthood (Thurlow et al. 2004:97). In comparison to the chameleonic notion of face, however, identity appears to be the more stable of the two concepts as it is face rather than identity which stresses the "centrality of human communication in forming and sustaining the social self" (Arundale 2006:200). Consequently, I side with Spencer-Oatey (2007:642), who considers identity to be relatively stable and enduring "cognitive representations" which are formed by speakers, telling them who they are.

This can be illustrated with the help of the following example: Those who have tried to rid themselves of bad habits or of unattractive character traits, such as smoking or making hasty judgments about people, know how hard it is to change one's way lastingly. While it is relatively easy to let others think that we have changed for the better (change of face), it is all the more difficult to allocate these alterations a permanent place within ourselves (change of identity). Not unlike a promotional poster, face is indeed "a public self-image" in the best Goffmanian sense, which means that it does not only encompass "what one thinks of oneself, but what one thinks others should think of one's worth" (Spencer-Oatey 2007: 642). Lines help us create this (momentary) image (used here synonymously with face), which depends on the hearer to be ratified in a dyadic process (see Figure 11). Our interlocutor, on the other hand, takes an opportunistic stance towards face constitution, since or although clues certainly do not superabound. In accordance with the assumption that we meet like-minded people on the Internet, with whom 
we share a great deal of our very own interests, views and values, face is assumed and holds until proven otherwise (Kendall 1999:66). It can thus be characterized as being comparatively more open for change, created to have an effect on our interlocutors, certainly based on our identities but not necessarily a truthful mirror thereof. Then again identity means our inner make-up, resting upon and solidified through a variety of influences and episodic and semantic memories of past experiences, which is only partly open to alteration.

Transferred to the domain of CMC, I proceed from the assumption that users do not, in fact, (re-)construct their own identities, as these are too deeply entrenched constructs within themselves to be altered just by a mouse-click. Instead, they constitute their online faces for the current purpose and goal of activity by changing their nickname, avatar or other superficial components of their online presence. As is often the case with technological innovations, the first explorative investigations ascribed almost magic potency and unprecedented possibilities of interaction to CMC. It was then that terms such as netspeak (Crystal 2001) and online identities were coined. With regard to the latter, Chandler (1998), for example, believed that the Web is a truly powerful "technology of self", a term used by Michel Foucault in 1988 to refer to the means with the help of which individuals represent themselves. Let us follow Chandler's (1998) train of thought yet a bit further: In the text-based world of CMC, in which people talk themselves into existence and thus construct their identities, these technologies of the self allow us not only to think about our identity and to transform the way we think of ourselves, but also to change ourselves to who we want to be. Turkle agrees and claims that

[y] ou can be whoever you want to be. You can completely redefine yourself if you want. You can be the opposite sex. You can be more talkative. You can be less talkative. Whatever. You can just be whoever you want really, whoever you have the capacity to be. You don't have to worry about the slots other people put you in as much. It's easier to change the way people perceive you, because all they've got is what you show them. They don't look at your body and make assumptions. All they see is your words.

(Turkle 1995: 184)

Meanwhile, however, we have learned not to overestimate the creative power of $\mathrm{CMC}$, which is why I consider the term identity construction (cf., for example, Locher 2011) as set too high and thus inappropriate. Instead, I prefer the notion of face constitution to designate more realistic processes of impression management in CMC.

Contrary to common belief, the relatively conscious creation of face is not a CMC-related innovation, but again an imitation of a procedure that is most natural in FtF interaction, as users unconsciously orient themselves by familiar 
FtF mechanisms: Goffman (cf. 4.3.2) compared social life in general to some kind of multi-staged drama in which each of us performs different roles in different social arenas (cf. Meyrowitz 1985:2). Just like social actors are constantly changing their costumes and roles, the presentation and texture of their faces (i.e. the facets they have shown) change accordingly, always with regard to new situations and audiences. For this reason, the aforementioned "dramaturgical element" of face constitution (cf. Coupland/Jaworski 2004:22) should not be overrated, as it is long since "old hat": Whether online or offline, the constant activity of representing ourselves to others accompanies us at every turn, on any stage of life.

Another inherent "item" that accompanies us wherever we go is those basic human needs which loom behind Brown/Levinson's bipartite concept of face (see Section 4.3.1). Logically, these needs for association and dissociation can also be spotted every so often in message board interaction. From a user's perspective, the need for association is evident in his getting involved to varying degrees, either by (constantly) contributing messages to threads, or by assuming responsibility in the board (for example as a moderator), by joining "societies" and by extending his buddy list, to give just a few examples. On the other hand, a user's need for dissociation is mirrored in behavior, which aims at keeping one's distance and thus guarding one's privacy and autonomy, for example by using nicknames and unrelated avatars or by refraining from disclosing (links to) private information (such as photos or contact details). Note, however, that avoiding affiliation is rather atypical for and counterproductive to the collaborative nature of message boards. This will also become evident in the empirical analysis to come.

Online codes of conduct urge their users, among other things, to respect their interlocutors' face needs (see Section 3.3.2). In changing the perspective and focusing on the interlocutor, we can detect that a user's need for association is met when their interlocutor welcomes them, interacts with them, invites them into a "society" or offers friendship. In Brown/Levinson's terms, this kind of behavior would be called positive politeness (see Section 4.3.1). Then again, this need can be threatened when interaction is withheld, users are ignored or, even worse, bullied or flamed (see Section 5.7). Finally, if interlocutors support a user's need for dissociation - thus showing negative politeness in Brown/Levinson's words - they respect his privacy, i.e. they accept nicknames and the like as they are. Not showing consideration for this need could be manifest in behavior which pressures a user to disclose more than he is willing to. In the worst case, interlocutors could even disclose information about someone single-handedly (such as photos, private mails, links etc.). The following example demonstrates the need for association and the ratification thereof: 
(1) Re: ¡Hola! Soy Lily.

Originally Posted by lilythrash 21

Hey, I stumbled upon this site last night and found it interesting, so I decided to make an account.

Allow me to introduce myself.

I'm Lily, and I'm a Cuban-American in Stamford, CT. I'm a musician,

a photographer, and a writer. I play bass, guitar, and I sing.

I'm not a very low-profile person. XD

Anyway, I hope to eventually become a part of this community. Can't wait to talk to some of you.

Hello Lily and Welcome to The Student Room

This place is very addictive! Enjoy your time here!.

Eventually become a part of this community.... You already are! $\oplus$

(RESPECT, thread \#2: post 2)

In her original post, which is quoted here by $R £ S P £ C T$, newcomer lilythrash 21 introduces herself and expresses her wish to "eventually become a part of this community". Her interlocutor replies by welcoming her with a typical smiley. He further indicates that she already is a part of the community, thereby attending to her positive face needs.

Instances of the need for dissociation can also be found in TSR message boards, as displayed in the next example, which shows an empty user profile. Although a regular user with a lot of reputation, not a single piece of personal information is disclosed. The user's privacy and distance to the community are thus protected:

(2) Toiletpaper8

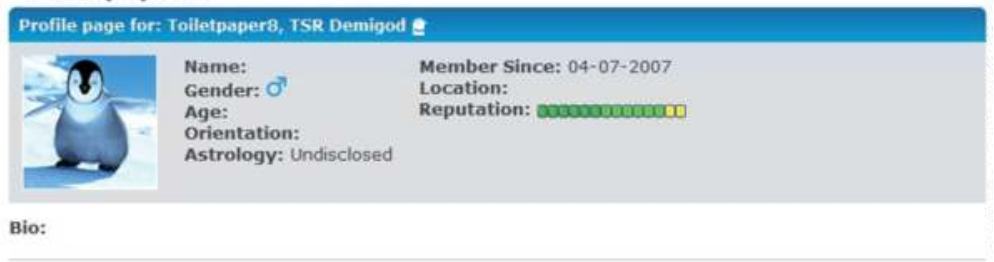

Where you study:

Academic Info:

Interests:

(Toiletpaper8, thread \#2: profile) 
The question that still needs to be answered, though, is how face is dynamically constituted and negotiated in TSR message boards in the best Goffmanian sense. Similar to FtF interaction, ${ }^{54}$ online face is also a bricolage of many diverse and partly ephemeral facets, a summative conglomerate which transpires through lines. Just as we spontaneously combine facets of our face for the current purpose in FtF interaction and make them known through our (non)verbal behavior (the lines), we can also piece together an online mosaic of our face - the only difference lies in the means involved: Technological gimmicks and templates lend themselves as vehicles for the transportation and publication of lines, which in turn serve as clues in the identification of a user's face claims. By altering and (re-)combining templates with a few clicks, we can constantly manipulate and remodel our lines and thus our face claims. Just like in FtF, we can thereby foreground one trait to the disadvantage of another. Unlike FtF, however, we certainly do this more consciously: In FtF, we are only partly in control of our lines due to time constraints and the simple fact that we are physically present and thus perceivable, to name but two among many other reasons. This process can be visualized in the following negotiation cycle (see Figure 15).

This interplay between lines dropped by users and interlocutors' as well as observers' possible line-based interpretations of their face claims shall be elucidated with the help of some examples.

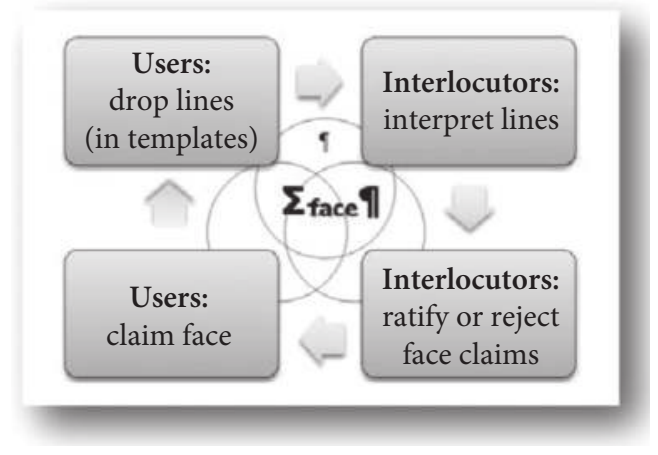

Figure 15. Cycle of face negotiation

54. But see O'Driscoll's objection “that participants' faces in mediated communication remain untouched at the time [since] production and reception of messages are dislocated not only in time but also in space" (2011:25f., original emphasis). This view is only partly shared. After all, facework does have effects on interlocutors, be they immediate as usually (but not always!) the case in $\mathrm{FtF}$ or lagged as in message boards. 
(3)

Tha_Black_Shinobi

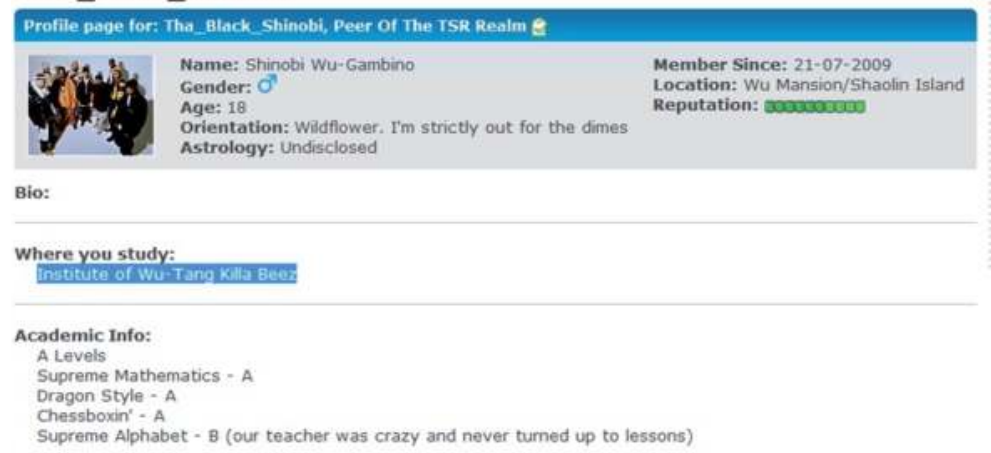

But seriously $I$ will hopefully be doing Law at the University of Nottingham in September If 1 Get 3 As

Interests:

Jesus, Hip Hop, Music, Films, girls, football, food, xbox and $i$ do a bit of reading here and there.

Extras

(Tha_Black_Shinobi, thread \#3: profile)

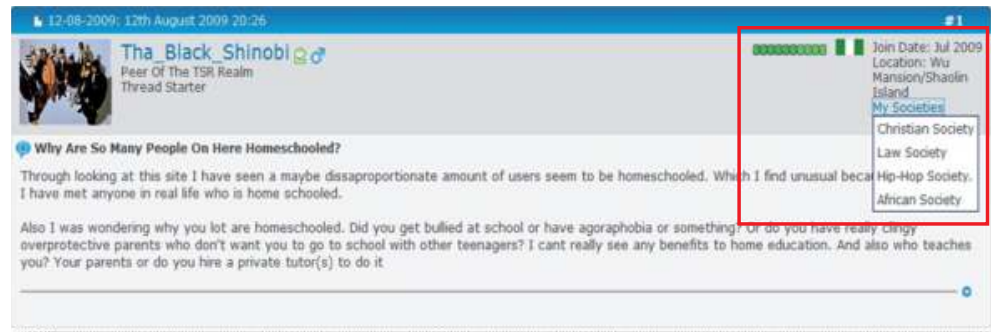

(Tha_Black_Shinobi, thread \#3: post 1)

This user's profile and posts exhibit a rather playful conduct with face consitution: His (or her, for that matter) self-portrayal shows a strong affiliation with a New York Hip Hop clan, the "Wu-Tang Clan", according to which he aligns (most of) his face claims. All the templates available to him, among them his nickname, avatar, signature, location information and flag as well as his societies ("Christian, Law, Hip Hop and African Society”), are used consistently to convey his major line, which could be phrased as either "I am a die-hard 'Wu-Tang Clan' fan" or even "I belong to the "Wu-Tang Clan"'. The latter interpretation of his lines can be deduced when looking at his signature, which shows a picture of said clan and is titled "My Family Portrait". Presumably winkingly, he fabricates a whole new online persona for himself. 
Further, maybe more authentic lines such as his religious and sporting streak can be derived from the section "Interests". With his statement in the lower section of his "Academic Info", he informs other users that "seriously [he] will hopefully be doing Law at the University of Nottingham in September [...]", thereby dropping another, maybe more truthful line about himself, which is insofar believable as it is backed up by his membership in the "Law Society". Above that, and even more importantly, this statement is an admission that most parts of his self-portrayal are not to be taken too seriously, which is, at least in this case, rather obvious.

This example of face constitution goes to show that lines cannot only lead to contradicting face claims and interpretations thereof ("I'm a Clan member" vs. "I'm a future student"), but also that lines do not necessarily have to add up to a coherent mosaic. Although the same holds also true for FtF interaction, the increased deceptive potential of online message boards, which is based on the technological possibilites, is nevertheless stressed by this example.

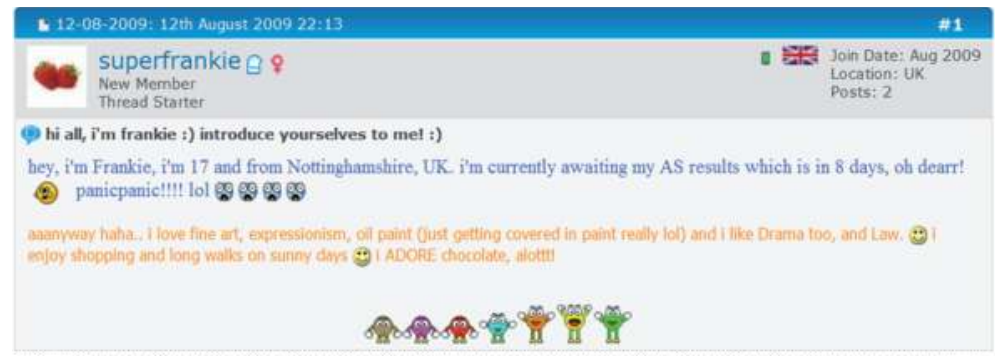

(superfrankie, thread \#1: post 1)

Picking up on the lines conveyed through various templates such as the label ("New Member"), the number of posts ("2"), the join date (with regard to the time stamp of the post) and the reputation system (only one gem), a rather coherent picture presents itself: superfrankie is an inexperienced newcomer, a newbie. In addition to this template-induced information, the content of her post as well as its rather idiosyncratic make-up further substantiate this first impression: She does not only utter explicit face claims about herself but drops more lines when she creatively garnishes these claims with abundant use of smileys, color changes, acronyms ("lol") and other in-group abbreviations ("AS"), capitalization (“ADORE”), repetitious punctuation (“!!!!”) and emulated speech ("aaanyway", "alottt"). Interpreting users could assume that superfrankie wants to be seen in a certain way, thus inferring the following face claims: UK, female, sociable, self-confident (see her nickname superfrankie), lively, emotional, casual (see the typos), young, versatile and probably many other things. Obviously, we do not 
always have to consult the profile, as some posts themselves are extremely revealing and full of lines. At least with regard to her self-confident and sociable nature, her profile information confirms this impression by proclaiming "i'm a bubbley, bright girl who loves to meet new people". The same is true for her versatility, as she lists plenty of interests in the respective section. Although consistent in her self-portrayal, she could, of course, exaggerate or masquerade her "true" face. Again we have to rely on the user to provide us with authentic information.

Whether online or offline, lines are indeed ratified by hearers - either explicitly or implicitly. Although most ratifications are tacit, some are indeed explicit. The following pair of examples bears witness to that fact:

(5) Re: ¡Hola! Soy Lily.

Originally Posted by lilythrash 21

Hey, I stumbled upon this site last night and found it interesting, so I decided to make an account.

Allow me to introduce myself.

I'm Lily, and I'm a Cuban-American in Stamford, CT. I'm a musician, a photographer, and a writer. I play bass, guitar, and I sing.

I'm not a very low-profile person. XD

Anyway, I hope to eventually become a part of this community. Can't wait to talk to some of you.

Hello. 9 You sound very talented. $:$

I hope you stick around. PM me if you need help or a chat.

(Kagutsuchi, thread \#2: post 6)

lilythrash21's introductory post, which also served as the starting point for example 1 (see above), is quoted and then commented on by another user, Kagutsuchi. By stating "You sound very talented", he interprets the line(s) which she consistently presented in her post as well as in her profile in her favor, thus explicitly ratifying her face claims.

Since lilythrash 21 chose to make her first appearance with a Spanish heading - she started her thread with the heading "Hola! Soy Lily!" - she dropped another line of her having a Spanish-speaking background and most likely being fluent in that language. This is also reinforced through her profile, where she describes herself with the words "I'm this Cuban-Irish-American number". 
Re: ¡Hola! Soy Lily.

Originally Posted by lilythrash 21

Ese debe ser el más breve mensaje de siempre.

(That must be the shortest message ever)

(Afcwimbledon2, thread \#2: post 21)

As this conversation unfolds, Afcwimbledon 2 picks up on her language choice and decides to formulate a reply to another one of her posts, a smiley, in Spanish as well, thereby implicitly ratifying her face claims.

\subsubsection{Trolling: A means of strategic deception}

Structures and templates available in message boards can also be exploited strategically for deceptive purposes, as information about our interlocutors is in most cases not verifiable. Deliberately misleading someone online is called trolling, a term which is borrowed from the domain of fishing. Trolling expert Donath explains this technique as follows:

[Y]ou set your fishing lines in the water and then slowly go back and forth dragging the bait and hoping for a bite. Trolling on the Net is the same concept someone baits a post and then waits for the bite on the line and then enjoys the ensuing fight.

(1999:45)

Trolling is thus a game in which participants regularly and intentionally bring the play with their face-masks to the boil. Unfortunately, this game is often played without everyone involved knowing about his participation - except for one person, of course, the deceiver. The "troll" 55 sets out to gain access to a certain group of people within a community, pretending to be one of many legitimate participants to share interests and values. He fools his interlocutors into believing his face claims by posting messages which are "intentionally incorrect but not overly controversial" (Herring et al. 2002:372), thus stirring up trouble from within. While flames aim at inciting all readers, the goal of a stereotypical troll is "to draw in particularly naive or vulnerable readers" and to catch inexperienced users (ibid.). Then again the result of both flaming and trolling are rather similar. According to Donath (1999:45), trolling cannot only disrupt or divert the

55. As noted by Herring et al. (2002:382), the terms "troll" and "troller" can be used interchangeably to refer to a person who sends messages which deliberately convey a wrong impression about themselves. Unfortunately, their messages are also called "trolls". 
discussion or disseminate bad advice. Above that, it can also damage the feeling of trust in a community, or create an atmosphere of constant suspicion. A troll can thus be extremely detrimental to interpersonal relations in online communities in such a way that simple and naive questions are not answered anymore, if everyone is suspected to be a troll anyway. Consequently, an ambience of mistrust and latent accusations can scare away newbies.

Although trolls indeed walk abroad one message board or the other, thereby personifying the malicious intent to veil one's true nature, basic claims about face deception in CMC in general and in message boards in particular as described above should be considered as the exception rather than rule. Burnett/Marshall (2003) rightly point out that early assumptions about the creative potential of "identity" play online were overgeneralizations based on particular CMC contexts like MUDs, in which the playful adoption of fantasy identities is their raison d'être. A more realistic stance is adopted by Döring (1999:38f.), who assumes that, despite technological possibilities to deceive, users prefer exchanges which are based on authentic facts rather than on creative fabrications. When we recall the motivation of users to go online and to participate in message boards in the first place, the saying "Birds of a feather flock together" springs to mind. As discussed before (see Chapter 3), the majority of users seek the casual get-together and exchange with like-minded people, to detect genuine commonalities and maybe even bond over shared topics. Deception thus appears highly inappropriate at least with regard to the accomplishment of this special social purpose. Besides, keeping up a façade is certainly much more exhausting than sticking to the facts.

\subsection{The relations: Interpersonal contextual factors}

The discussion of face deception online already involved another important component of interaction other than the speaker: the interlocutor. As these two belong together like two sides of the same coin, the focus is now shifted from the (self-portrayal of the) individuals to their relations with their interlocutors and their manifestations in TSR message boards. With the exception of the first two questions, which have already been answered in the previous part, Arndt/ Janney's (2005:32f.) complex of questions fathoms the second dimension rather appropriately:

What kind of partner am I dealing with? What kind of person does the partner think I am? Are we equal or unequal? Do we like each other or not? Is the partner a member of group with which I identify myself? What is the partner's role in 
the group? What do we have in common? How does the partner view our social relationship? What are the partner's motives? How does the partner expect me to talk? What strategies have worked in the past with partners like this?

Accordingly, we need to take the following factors, which determine interpersonal relations, into consideration:

- social distance between interlocutors;

- power relations between interlocutors;

- social norms shared between interlocutors;

- content-related and interpersonal motives;

- participation structure;

- previous exchanges (common ground).

Previous exchanges hold an exceptional position insofar as they do not only provide an important basis for interlocutors' common ground and necessary co-text for the evaluation of utterances. Above that, they also lend themselves as a source for the determination of all the other factors on the list. It becomes once more obvious that all these interpersonal contextual factors cannot be viewed as operating detached from each other but rather as small cogs in a big wheel, constantly influencing one another. Similar to personal contextual factors, interpersonal relations and practices are also established in message boards by means of continued message exchange but also along the lines of experiences made in FtF interactions. Only in those rare cases in which interlocutors have created and defined their relations in real life before, is it theoretically possible to transfer these relations to the online domain. The distinction of these two cases, let alone the detection of shades of grey in-between, lies again beyond the capacity of observing outsiders.

The first two items on the list, social distance and power relations can be described as multidimensional, constantly evolving and interacting concepts. With recourse to Brown/Levinson's (1978) outline of the two contextual factors $\mathrm{D}$ (distance) and $\mathrm{P}$ (power), we can characterize the former as symmetric, horizontal and the latter as asymmetric, vertical relations (see Section 4.3.1). Social distance, which is also known under various aliases such as solidarity, closeness, familiarity and relational intimacy (Spencer-Oatey 2000b:33), is dependent on factors like social similarities, frequency of contact and length of acquaintance, which can create "likemindedness or similar behavior dispositions" (Brown/ Gilman 1972:258).

Then again power is sometimes labeled status, dominance or authority (Spencer-Oatey 2000b:32) and alludes to the degree to which one person is able to control the behavior of and impose on the other. Watts further explains that "A exercises power over B when A affects B in a manner contrary to B's initially 
perceived interests, regardless of whether B later comes to accept the desirability of A's actions" (1991:62). Accordingly, power is a relationship between at least two people, which is not reciprocal in the sense that both can have power in the same area of behavior (Brown/Gilman 1972:255). It is interesting to note that, especially in the case of power, relational work and power are mutually influential. Determining directions of causality reasonably is thus rendered almost impossible. An integral part of the concept of power certainly are those (expectations of) rights and obligations that come with the territory of social roles within a CoP such as TSR (see Section 3.3.1). Social online roles are dynamically renegotiated among participants based on "the extent and quantity of participation rather than [on] social pre-givens" (Avgerinakou 2003:281).

Since item number three on the above list, shared norms, has already been dealt with in detail in Section 3.3.2, we can focus on content-related and interpersonal motives for social encounters in TSR boards right away. Following Tomasello (2008: 136), we are animated to interact with each other online or offline by three fundamental motives: requesting (things, information), sharing (emotions, opinions) and informing others, which can be done for recreational, professional, pedagogical, creative and many other communication purposes (Herring 2001:622). Of course, these motives are closely linked, if not even directly related to interpersonal aims as well. All of these motives can be dealt with in more or less detail in various topical discussions provided by the hierarchy of subtopics and related threads within TSR. Depending on the current purpose of interaction, interlocutors naturally invoke certain expectations, which help them evaluate the appropriateness of certain utterances.

Finally, the item participant structure aims to amass those demographics of the CoP which might also be influential for interpersonal group processes. We are therefore looking for clues concerning, for example, the distribution of gender, the average age, the sum total of ratified members (both actually, actively participating as well as potential members), the amount and rate of participation and the like. All of these aspects will be fathomed in the empirical part of this study (see Section 7.2).

Most of these interpersonal contextual factors find expression in the same technological means of TSR that have already been discussed above in the context of self-representation of participants. The contents and make-up of current and previous posts, including all of their central and peripheral templates (with signatures, labels and societies leading the way), thus also appear as a major source for the retrieval of interpersonal information. Above that, however, registered users have access to "buddy lists", which mirror online friendships. Furthermore, FAQs have already proven useful as a source for normative views on behavior in TSR (see Section 3.3.2). 


\subsection{The setting: (Extra-) medial environmental factors}

Last but not least, some thoughts about environmental factors are still in order. To cut a long story short, this third type of factors, which can have an impact on interlocutors' evaluations of utterances, encompasses basically anything that can be pointed or referred to. With regard to the offline world, pointing is to be taken literally, while referring to something online can also be carried out with the help of hyperlinks or embedded objects such as photos, graphics, videos, sound files and the like. It could be argued, however, that hyperlinks are not variables within a user's environment themselves but rather textual, cohesive means to relate a source domain with a target domain. Only the latter of the two and thus part of the hyperlink is a piece of the user's environment to be pointed at. R. Jones remarks that "the physical circumstances in which computer mediated communication takes place can have important effects on how such interaction is conducted" (2002:3). In this way, almost everything and everybody in the real or virtual environment can become influential, if turned into context by interlocutors. Needless to say that contextualized resources from the offline world can hardly be traced from the interlocutor's and the observer's online perspective, unless made explicit. For this reason, R. Jones criticizes the use of the notion setting as it "is too static and material to adequately capture the dynamic, contingent and expansive interaction of material and virtual realities involved in computer mediated communication" (2002:11) and considers the German term Umwelt the more appropriate choice to capture practically anything that surrounds social actors during the process of interaction.

\subsection{Summary: A comprehensive framework of context in message boards}

Obviously, there is a vast number of (personal and interpersonal) contextual as well as environmental factors which can - at least in theory - be held responsible for the highly individual allocation of evaluative labels for instances of relational work. As outside observers and also as interlocutors, or even speakers for that matter, we can never be sure which factor was indeed pivotal for a certain value judgment, since we are dealing with a multifaceted and unpredictable interplay of a diverging number of partly highly ephemeral factors (see Table 12).

Although I have argued that message board templates make a noticeable effort to provide contextual information, a word of warning still is in order: (1) Templates, independent of their number and degree of sophistication, cannot work miracles, which is why a lot of contextual factors still remain in the dark or would have to be filtered from message contents in extremely elaborate processes - with 
Table 12. Contextual and environmental variables in message boards

\begin{tabular}{l|l}
\hline Domain & Factors \\
\hline & $\begin{array}{l}\text { - appearance: voice, bodily features, clothes etc. } \\
\text { - demographics: gender, age, ethnicity, social class, educational } \\
\text { level, occupation, status, region etc. } \\
\text { - current feelings, moods etc. } \\
\text { - cultural background } \\
- \text { knowledge gathered in past experiences }\end{array}$ \\
\hline
\end{tabular}

no prospect of success in terms of accuracy and validity. But everything is not lost as users, following Suler's (2004:n.p.) line of argumentation, are quite savvy in deducing missing contextual information and filling in missing clues, always carried by the wish to have found a likeminded soul. (2) The danger of circular argumentation is looming when it comes to determining evaluations of relational work based on contextual factors and vice versa! This becomes alarmingly evident in Morand/Ocker's (2003:n.p.) statement about role orientations: "The specific tactics of politeness may thus prove useful to researchers in measurement of these role orientations." The following analysis will thus treat contextual factors as helpful indices for the evaluation of relational work without falling into the trap of overinterpreting template-mediated clues. 


\title{
Analyzing online message boards I
}

\author{
Thread starts
}

\subsection{Introduction}

It is the lack of direct correspondence between text and discourse that makes communication so indeterminate, and so intriguing.

(Widdowson 2007:8)

The discussion of relational work in previous chapters has shown that (the meaning of) discourse cannot be read off unambiguously from text, since it is cooperatively and spontaneously created and negotiated by interlocutors - in Widdowson's words, "when we use language we do not just present the meanings that are encoded in it, we exploit them as a potential resource for making meaning of our own [thus making] semantic meaning serve a pragmatic purpose" (2007:8). In this context, Herring warns that the textual basis "can only tell us what people do (and not what they really think or feel). Any interpretations of the latter based on the former necessarily contain an element of speculation and risk being incorrect" (2004:358). The speculative and risky business of interpreting texts can, however, be diminished to at least some extent by incorporating contextual factors and metapragmatic utterances, which both have the potential to enhance the accuracy of the allocation of form and function. ${ }^{56}$

While contextual factors have been discussed in great detail in the previous chapter, a few elucidations on metapragmatic utterances are still in order. Caffi defines metapragmatics, among others, as "the investigation of that area of the speaker's competence which reflects the judgments of appropriateness on one's own and other people's communicative behavior" (1993:2461). Accordingly, metapragmatic utterances refer to speakers' quotes or comments on their own, someone else's, or the speech community's use of language (Hongladarom 2007:32).

56. Another way of backing up interpretative analysis would be to ask users about their underlying intentions. As argued in previous chapters (see Section 5.5), more often than not users are not at all capable of pinpointing the exact nature of their doing, viz. their intention, neither in retrospect nor during the actual conversation. For this reason, this study desists from interviewing participants and bases its argumentation on the textual documentation alone. 
Realistically, however, we will not encounter helpful contextual factors and/or enlightening metapragmatic utterances at every step of the way throughout the analysis. Still, the investigation of interpersonal relations in message board entries can be promoted by adopting a favorable perspective, viz. as participant observer. Originated by social anthropologists such as Malinowski (1922), who also coined the term participant observation, this traditional and renowned research strategy has developed into the principal method for ethnographic research and has been used in a wide range of disciplines, among them sociology, communication studies, social psychology but also in linguistics (see, for example, studies by Holly (1979) or Beißwenger (2010)). The analyst becomes or at least takes the stance of a participating observer who does not merely observe, but tries to find a role within the group he observes - even if only as outside observer. In putting himself in the shoes of his objects of study - in this case, message board participants - he hopes that his evaluations mirror those of actual participants.

In any event, one can argue that message boards and other forms of CMC have known participant observers for a long time and even recruit actual users from them: they are called lurkers (see Section 3.3.1), who sometimes follow the proceedings of a single thread or an entire board religiously until they either decide to get actively involved or lose interest. These participant observers, including the analyst of this study, are as familiar with the thread and its contributors as any other user and are thus absolutely capable of valid value judgments. Since participants in most CMC settings are usually aware of the possible and even probable presence of lurkers, the observer's paradox is of no consequence. Of course, "official" online participants and participant observers rely on slightly divergent pools of contextual information, with participant observers intentionally looking for as many contextual clues as possible and "official" participants sharing more episodic knowledge. Still, this fact is also rather unproblematic - after all this is a characteristic feature of communication in general, be it in FtF settings or online. Similar to FtF, we can act on the assumption that the overlap between these two pools is big enough to ensure somewhat identical evaluations on both sides (see also Section 5.3).

With the intention of getting a realistic picture of the structure of interpersonal relations on "the Internet", only a handful of studies have been conducted in the recent past. ${ }^{57}$ In general, Herring criticizes that

57. See the 2010 special issue of the Journal of Politeness Research containing, among others, Locher's general synopsis of the study of (interpersonal relations in) CMC in the introduction. 
$[\ldots]$ much research on online behavior is anecdotal and speculative, rather than empirically grounded. Moreover, Internet research often suffers from a premature impulse to label online phenomena in broad terms, e.g., all groups of people interacting online are 'communities'; the language of the Internet is a single style or 'genre'.

(2004:338)

Although mostly investigating authentic online interaction in one form of computer-mediated communication or the other, such as Internet lists (Herring 1995; Maricic 2001), email discussions (Harrison 2000; Hatipoglu 2007), instant messaging/chats (Darics 2010; Savas 2011), voice mail (Hobbs 2003), YouTube postings (Lorenzo-Dus et al. 2011), news sites (Neurauter-Kessels 2011) and even bulletin board systems (Simmons 1999) and fora (Lee 2005), most studies nevertheless exhibit certain weaknesses which do not only limit their general persuasiveness but also their value as a role model for this present study. While some of them concentrate on one aspect of interpersonal relations alone, like flaming as in Lee (2005) or identity construction (Erickson 1996; Taylor 2000; Baker 2001; Benwell/Stokoe 2006), most of them can be accused of showing too strong a bond with Brown/Levinson's original model when trying to apply (parts of) their system of categories to CMC data.

Against this background, the present study, which is to my knowledge unprecedented in this form, tries to steer clear of the aforementioned analytical traps. In trying to find empirically valid insights for one form of CMC alone, a corpus containing a cross section of the message board system of The Student Room (cf. Chapter 2 and 3) is investigated mostly qualitatively, keeping a safe distance from Brown/Levinson's classification. In terms of the validity of these results, a word of warning still is in order: Although the results gained in this study may exhibit some general trends and communicative mechanisms, they still represent nothing but a snapshot, as these findings are only true for the excerpts investigated at this specific point of time under these specific circumstances. Since every sample is unique, we should not assume the findings to be representative of TSR as a whole, message boards in general, let alone "the Internet".

The aim of this chapter is hence twofold: describing the inner make-up and the compilation of the corpus first, before conducting the first part of the empirical analysis, which is supposed to shed some light on how interpersonal relations are established in this environment. In order to do so, the focus will be put on thread starts, viz. first entries, alone. After the investigation of the content and structure of their headings, the users behind these first entries will be introduced. The third and most important component of this chapter is, however, dedicated to the formal and functional description of actual thread starts. 


\subsection{Aspects of corpus compilation and its internal structure}

It should go without saying that the mere process of compiling a corpus - at first totally independent of its analysis - is an endeavor which is just as biased as the judgment of interpersonal relations itself since corpora are always designed with a certain purpose in mind (cf. Schulze 1985: 119). Consequently, it is important to shortly outline those aspects that influenced the compilation of the respective corpus: For the empirical analysis targeted in this study, I was looking for material which involved "typical" message board users in "typical", non-specific topical communication. According to S. G. Jones' report "The Internet Goes to College" (2002), which documents a large-scale survey of Internet use among several thousand university and college students across the United States, students appear as heavy users of the Internet in comparison to the general population. As S. G. Jones' study revealed, using the Internet is completely integrated into college students' daily routines and is as much a tool for educational as it is for interpersonal purposes. With this in mind, a message board which is predominantly populated by students took center stage in the first process of selection: The Student Room (henceforth TSR). Beside its appealing target group of British and international students ${ }^{58}$ and its topical variety, it also qualified because of its structural features (see Chapters 2 and 6), which disclose a lot of additional, valuable information about the context and the participants.

Above that and every bit as important as the aforementioned aspects, TSR also met essential ethical considerations. As message board conversations can be read long after they actually took place, they are a hybrid between interpersonal and mass communication (Marcoccia 2004:117, see also Section 3.3.1). This raises an ethical issue, including concerns about (personal) copyright, which orbits around the much discussed question what kind of online material can be exploited unhesitatingly for scientific analyses. It is thus important to note that for the purpose of this study, every single piece of input for the corpus - messages as well as surrounding interpersonal information - was and still is completely open to public. It can be accessed unhindered, since TSR does not require observers to register as a member in order for them to read through its threads. Claridge (2007: 88) compares fora in general (thus also message boards) to "normal' web sites [which] can be visited and read by any Internet user at any time, in the same way as people would read an online newspaper" - a view which is also shared by Graham (2010) and Holmberg (2008). Tanskanen views message board discussions "as unproblematic in this respect" (2007: 89). For the purpose of this study, I

58. Although message boards may be designed and intended to attract a certain crowd, theoretically they are open to practically everybody interested (cf. Claridge 2007:94). 
adopted Maricic's (2005: 71f.) standards, according to which participants' consent for the usage of their discourse is not required if

- the data are gathered from a public forum which requires neither password nor registration in order to access it;

- the participants do not represent a group of people in a potentially vulnerable position (such as minorities of any kind);

- the thread does not deal with topics that could possibly damage the participants if exposed.

In view of the vagueness of the last of the three items on the list, Maricic (2005:72) proposes to disguise anything that might disclose participants' real identities, just to be on the safe side.

Once the appropriate message board had been chosen, the corpus could be compiled. From the various data sampling techniques proposed by Herring (2004:348), I decided for a random mode of thread selection within TSR, meaning that "each [thread was] selected or not by a coin toss" (ibid.) within various subtopics, thus guaranteeing representativeness and generalizability. The usual disadvantage of this course of action, according to Herring loss of context and coherence, could be counteracted by downloading complete and coherent threads instead of single, decontextualized messages. The term complete has to be handled with care, though. Threads always have a clear beginning as they come into existence the moment a user, the thread starter, decides to introduce a new topic. They do not, however, end in a pre-determined way, but simply "die" without any warning due to lack of interest (Claridge 2007:91), a process which can take hours, days, months or even years. Since threads can always be taken up again, even after a sizable amount of time with no activity at all, it is practically impossible to tell whether a thread is "complete" or not. ${ }^{59}$ Consequently, completeness could not be taken as a defining criterion for the choice of a thread. Instead, deadlines were set for the acquisition of samples (see Table 13 below): all the posts within the thread available up to this point in time were collected in their entirety. However, a look at the sampling deadline in comparison to the date of the last entry shows that in most cases, users had already lost interest in the topic some time before the download date. This means that the latest discussions must have been brought to a preliminary end, hence temporarily concluding the entire thread.

59. A classic example of this phenomenon can be found in thread \#5 (Meteor Shower :)): Started in 2008 (07/08/2008), it was quite attractive as a topic for two days before it died down. It was picked up again and continued, however, almost exactly one year later (12/08/2009) - again staying interesting for two days before dying down once more. Another thread which was carried forward for several years was \#38 (Jolly useful Geography websites). 
To enable easy processing, the complete material gathered in this way was saved as screenshots to ensure visual cohesion and in the format of word files to facilitate subsequent work with the text. At the end of the sampling process, the corpus comprised 50 threads, featuring the following details (see Table 13). ${ }^{60}$

Table 13. Internal structure of the message board corpus

\begin{tabular}{|c|c|c|c|c|c|c|c|}
\hline \# & Thread title & $\begin{array}{l}\text { No. } \\
\text { posts }\end{array}$ & $\begin{array}{c}\text { No. } \\
\text { users }\end{array}$ & $\begin{array}{l}\text { Date of first } \\
\text { \& last post }\end{array}$ & $\begin{array}{l}\text { No. } \\
\text { days }\end{array}$ & $\begin{array}{c}\text { Posts/ } \\
\text { day }\end{array}$ & $\begin{array}{l}\text { Sampling } \\
\text { deadline }\end{array}$ \\
\hline 1 & $\begin{array}{l}\text { hi all, i’m frankie :) introduce } \\
\text { yourselves to me! :) }\end{array}$ & 18 & 8 & $\begin{array}{l}12 / 08 / 2009- \\
15 / 08 / 2009\end{array}$ & 4 & 4.5 & $01 / 10 / 2009$ \\
\hline 2 & ¡Hola! Soy Lily. & 42 & 10 & $\begin{array}{l}05 / 08 / 2009- \\
11 / 08 / 2009\end{array}$ & 7 & 6.0 & $01 / 10 / 2009$ \\
\hline 3 & $\begin{array}{l}\text { Why Are So Many People On Here } \\
\text { Homeschooled? }\end{array}$ & 81 & 49 & $\begin{array}{l}12 / 08 / 2009- \\
13 / 08 / 2009\end{array}$ & 2 & 40.5 & $01 / 10 / 2009$ \\
\hline 4 & booze illegal? & 67 & 37 & $\begin{array}{l}13 / 08 / 2009- \\
14 / 08 / 2009\end{array}$ & 2 & 33.5 & $01 / 10 / 2009$ \\
\hline 5 & Meteor Shower :) & 74 & 45 & $\begin{array}{l}07 / 08 / 2008- \\
13 / 08 / 2009\end{array}$ & 7 & 10.6 & $01 / 10 / 2009$ \\
\hline 6 & Are you in favour of Euthanasia? & 67 & 49 & $\begin{array}{l}11 / 08 / 2009- \\
13 / 08 / 2009\end{array}$ & 3 & 22.3 & $01 / 10 / 2009$ \\
\hline 7 & $\begin{array}{l}\text { Who are more friendly: Northerners } \\
\text { or Southerners (England) }\end{array}$ & 120 & 90 & $\begin{array}{l}11 / 08 / 2009- \\
13 / 08 / 2009\end{array}$ & 3 & 40.0 & $01 / 10 / 2009$ \\
\hline 8 & 'Life is too short' & 57 & 31 & $\begin{array}{l}10 / 08 / 2009- \\
13 / 08 / 2009\end{array}$ & 4 & 14.3 & $02 / 10 / 2009$ \\
\hline
\end{tabular}

\begin{tabular}{|c|c|c|c|c|c|c|c|}
\hline 9 & $\begin{array}{l}\text { Is TSR an accurate reflection of today's } \\
\text { youth? }\end{array}$ & 34 & 31 & $\begin{array}{l}12 / 08 / 2009- \\
12 / 08 / 2009\end{array}$ & 1 & 34.0 & $02 / 10 / 2009$ \\
\hline 10 & Could you ever forget your family? & 112 & 39 & $\begin{array}{l}12 / 08 / 2009- \\
13 / 08 / 2009\end{array}$ & 2 & 56.0 & $02 / 10 / 2009$ \\
\hline 11 & $\begin{array}{l}\text { What does this girl mean by this? } \\
\text { (convo included) }\end{array}$ & 120 & 92 & $\begin{array}{l}13 / 08 / 2009- \\
15 / 08 / 2009\end{array}$ & 3 & 40.0 & $02 / 10 / 2009$ \\
\hline 12 & I'm bitter and twisted & 66 & 36 & $\begin{array}{l}12 / 08 / 2009- \\
13 / 08 / 2009\end{array}$ & 2 & 33.0 & $02 / 10 / 2009$ \\
\hline 13 & Do intellectuals put you off? & 65 & 35 & $\begin{array}{l}13 / 08 / 2009- \\
13 / 08 / 2009\end{array}$ & 1 & 65.0 & $02 / 10 / 2009$ \\
\hline 14 & $\begin{array}{l}\text { How to stop strange people speaking } \\
\text { to me }\end{array}$ & 54 & 41 & $\begin{array}{l}13 / 08 / 2009- \\
14 / 08 / 2009\end{array}$ & 2 & 27.0 & $02 / 10 / 2009$ \\
\hline
\end{tabular}

6o. As noted before, spelling and grammar mistakes in the original posts, headings etc. remained unchanged. 
Table 13. (continued)

\begin{tabular}{|c|c|c|c|c|c|c|c|}
\hline$\#$ & Thread title & $\begin{array}{l}\text { No. } \\
\text { posts }\end{array}$ & $\begin{array}{l}\text { No. } \\
\text { users }\end{array}$ & $\begin{array}{l}\text { Date of first } \\
\text { \& last post }\end{array}$ & $\begin{array}{l}\text { No. } \\
\text { days }\end{array}$ & $\begin{array}{c}\text { Posts/ } \\
\text { day }\end{array}$ & $\begin{array}{l}\text { Sampling } \\
\text { deadline }\end{array}$ \\
\hline 15 & To drink or not to drink....? & 67 & 54 & $\begin{array}{l}12 / 08 / 2009- \\
01 / 09 / 2009\end{array}$ & 21 & 3.2 & $02 / 10 / 2009$ \\
\hline 16 & $\begin{array}{l}\text { What does it mean if a girl smiles at } \\
\text { you? }\end{array}$ & 61 & 52 & $\begin{array}{l}11 / 08 / 2009- \\
13 / 08 / 2009\end{array}$ & 3 & 20.3 & $2 / 10 / 2009$ \\
\hline 17 & $\begin{array}{l}\text { Burton's rejected me for a credit card, } \\
\text { I think:S }\end{array}$ & 23 & 12 & $\begin{array}{l}12 / 08 / 2009- \\
13 / 08 / 2009\end{array}$ & 2 & 11.5 & $02 / 10 / 2009$ \\
\hline 18 & $\begin{array}{l}\text { The Official 'I'm going to fail my } \\
\text { exams' Society }\end{array}$ & 172 & 123 & $\begin{array}{l}18 / 05 / 2005- \\
24 / 06 / 2009\end{array}$ & 1498 & 0.1 & $02 / 10 / 2009$ \\
\hline 19 & Snow Patrol!!! & 75 & 24 & $\begin{array}{l}30 / 11 / 2006- \\
19 / 08 / 2009\end{array}$ & 1115 & 0.1 & $02 / 10 / 2009$ \\
\hline 20 & Most underrated Actor? & 84 & 70 & $\begin{array}{l}03 / 08 / 2009- \\
08 / 09 / 2009\end{array}$ & 6 & 14.0 & $02 / 10 / 2009$ \\
\hline 21 & Half Blood Prince thread ${ }^{\star}$ no spoilers ${ }^{\star}$ & 143 & 86 & $\begin{array}{l}15 / 07 / 2009- \\
10 / 08 / 2009\end{array}$ & 27 & 5.3 & $03 / 10 / 2009$ \\
\hline 22 & Favourite Musical and Why? & 49 & 34 & $\begin{array}{l}03 / 06 / 2009- \\
31 / 08 / 2009\end{array}$ & 90 & 0.5 & $03 / 10 / 2009$ \\
\hline 23 & $\begin{array}{l}\text { Who's your favourite Friends } \\
\text { character? }\end{array}$ & 69 & 53 & $\begin{array}{l}26 / 07 / 2009- \\
06 / 08 / 2009\end{array}$ & 10 & 6.9 & $03 / 10 / 2009$ \\
\hline 24 & $\begin{array}{l}\text { Would you go on holiday with some- } \\
\text { one from TSR you don't know irl? }\end{array}$ & 44 & 35 & $\begin{array}{l}17 / 07 / 2009- \\
14 / 08 / 2009\end{array}$ & 29 & 1.5 & $03 / 10 / 2009$ \\
\hline 25 & What countires have you been to? & 120 & 104 & $\begin{array}{l}05 / 08 / 2009- \\
25 / 08 / 2009\end{array}$ & 21 & 5.7 & $03 / 10 / 2009$ \\
\hline 26 & $\begin{array}{l}\text { Has anyone been to America recently } \\
\text { ??...border control experience }\end{array}$ & 86 & 54 & $\begin{array}{l}27 / 07 / 2009- \\
10 / 08 / 2009\end{array}$ & 15 & 5.7 & $03 / 10 / 2009$ \\
\hline 27 & $\begin{array}{l}\text { Why modern feminism is illogical, } \\
\text { unnecessary, and evil }\end{array}$ & 87 & 21 & $\begin{array}{l}03 / 08 / 2009- \\
12 / 08 / 2009\end{array}$ & 10 & 8.7 & $03 / 10 / 2009$ \\
\hline 28 & Women Make Crappy Musicians & 88 & 69 & $\begin{array}{l}21 / 07 / 2009- \\
31 / 08 / 2009\end{array}$ & 42 & 2.1 & $03 / 10 / 2009$ \\
\hline 29 & Creationism of evolution? & 100 & 30 & $\begin{array}{l}17 / 06 / 2009- \\
01 / 10 / 2009\end{array}$ & 107 & 0.9 & $03 / 10 / 2009$ \\
\hline 30 & $\begin{array}{l}\text { how much of the bible is 'truth' and } \\
\text { can god really have made everything? }\end{array}$ & 80 & 25 & $\begin{array}{l}18 / 03 / 2009- \\
22 / 03 / 2009\end{array}$ & 5 & 16.0 & $03 / 10 / 2009$ \\
\hline 31 & Should governments block websites? & 31 & 24 & $\begin{array}{l}25 / 02 / 2008- \\
16 / 08 / 2009\end{array}$ & 538 & 0.06 & $03 / 10 / 2009$ \\
\hline 32 & $\begin{array}{l}\text { Are we waling into a surveillance } \\
\text { society? }\end{array}$ & 49 & 26 & $\begin{array}{l}10 / 08 / 2009- \\
12 / 08 / 2009\end{array}$ & 3 & 16.3 & $03 / 10 / 2009$ \\
\hline
\end{tabular}


Table 13. (continued)

\begin{tabular}{|c|c|c|c|c|c|c|c|}
\hline$\#$ & Thread title & $\begin{array}{l}\text { No. } \\
\text { posts }\end{array}$ & $\begin{array}{l}\text { No. } \\
\text { users }\end{array}$ & $\begin{array}{l}\text { Date of first } \\
\text { \& last post }\end{array}$ & $\begin{array}{l}\text { No. } \\
\text { days }\end{array}$ & $\begin{array}{c}\text { Posts/ } \\
\text { day }\end{array}$ & $\begin{array}{l}\text { Sampling } \\
\text { deadline }\end{array}$ \\
\hline 33 & What makes you happy? & 104 & 90 & $\begin{array}{l}05 / 12 / 2008- \\
26 / 09 / 2009\end{array}$ & 296 & 0.4 & $03 / 10 / 2009$ \\
\hline 34 & Pros and cons of boarding school & 15 & 09 & $\begin{array}{l}01 / 05 / 2009- \\
12 / 08 / 2009\end{array}$ & 103 & 0.1 & $04 / 10 / 2009$ \\
\hline 35 & $\begin{array}{l}\text { Do you use a timetable to study? Or } \\
\text { don't you even plan?? }\end{array}$ & 33 & 31 & $\begin{array}{l}06 / 08 / 2009- \\
07 / 08 / 2009\end{array}$ & 2 & 16.5 & $04 / 10 / 2009$ \\
\hline 36 & Guide to How to Revise Effectively? & 42 & 31 & $\begin{array}{l}22 / 05 / 2008- \\
30 / 08 / 2009\end{array}$ & 466 & 0.09 & $04 / 10 / 2009$ \\
\hline 37 & $\begin{array}{l}\text { Grammar schools beat the private } \\
\text { sector. }\end{array}$ & 56 & 33 & $\begin{array}{l}09 / 08 / 2009- \\
13 / 08 / 2009\end{array}$ & 5 & 11.2 & $04 / 10 / 2009$ \\
\hline 38 & Jolly useful Geography websites & 88 & 49 & $\begin{array}{l}12 / 10 / 2004- \\
20 / 09 / 2009\end{array}$ & 1804 & 0.05 & $04 / 10 / 2009$ \\
\hline 39 & $\begin{array}{l}\text { music /noise whilst staying at uni } \\
\text { halls? }\end{array}$ & 19 & 13 & $\begin{array}{l}12 / 08 / 2009- \\
15 / 08 / 2009\end{array}$ & 4 & 4.8 & $04 / 10 / 2009$ \\
\hline 40 & Veganism & 27 & 22 & $\begin{array}{l}25 / 03 / 2009- \\
11 / 08 / 2009\end{array}$ & 140 & 0.2 & $04 / 10 / 2009$ \\
\hline 41 & $\begin{array}{l}\text { So it seems everyone just wants to ruin } \\
\text { there livers in university }\end{array}$ & 39 & 32 & $\begin{array}{l}10 / 08 / 2009- \\
15 / 08 / 2009\end{array}$ & 6 & 6.5 & $04 / 10 / 2009$ \\
\hline 42 & $\begin{array}{l}\text { Your pearls of wisdom to university } \\
\text { freshers... }\end{array}$ & 108 & 75 & $\begin{array}{l}03 / 08 / 2009- \\
18 / 08 / 2009\end{array}$ & 16 & 6.8 & $04 / 10 / 2009$ \\
\hline 43 & Image change when going to uni? & 50 & 48 & $\begin{array}{l}08 / 08 / 2009- \\
11 / 08 / 2009\end{array}$ & 4 & 12.5 & $04 / 10 / 2009$ \\
\hline 44 & $\begin{array}{l}\text { Am I wasting my time studying } \\
\text { English? }\end{array}$ & 31 & 14 & $\begin{array}{l}26 / 05 / 2009- \\
12 / 08 / 2009\end{array}$ & 79 & 0.4 & $04 / 10 / 2009$ \\
\hline 45 & Best universities for english? & 38 & 25 & $\begin{array}{l}15 / 07 / 2009- \\
22 / 07 / 2009\end{array}$ & 8 & 4.8 & $04 / 10 / 2009$ \\
\hline 46 & $\begin{array}{l}\text { Can studying literature become too } \\
\text { pretentious? }\end{array}$ & 64 & 40 & $\begin{array}{l}18 / 06 / 2009- \\
03 / 07 / 2009\end{array}$ & 16 & 4.0 & $04 / 10 / 2009$ \\
\hline 47 & $\begin{array}{l}\text { The best universities for film and } t v \\
\text { production? }\end{array}$ & 42 & 27 & $\begin{array}{l}05 / 01 / 2009- \\
07 / 09 / 2009\end{array}$ & 245 & 0.2 & $04 / 10 / 2009$ \\
\hline 48 & Q\&A thread - Journalism Courses & 22 & 6 & $\begin{array}{l}02 / 05 / 2009- \\
08 / 05 / 2009\end{array}$ & 7 & 3.1 & $04 / 10 / 2009$ \\
\hline 49 & Gap Year vs Career...Me vs Parents & 18 & 10 & $\begin{array}{l}29 / 07 / 2009- \\
31 / 07 / 2009\end{array}$ & 3 & 6.0 & $04 / 10 / 2009$ \\
\hline 50 & 'Tories plan tougher teacher tests' & 70 & 26 & $\begin{array}{l}02 / 07 / 2009- \\
02 / 10 / 2009\end{array}$ & 93 & 0.8 & $04 / 10 / 2009$ \\
\hline
\end{tabular}


Table 14. Sum total of contributions per user (C) in relation to their occurrence (O)

\begin{tabular}{lllllllllllll}
\hline $\mathrm{C}$ & $\mathbf{1}$ & $\mathbf{2}$ & $\mathbf{3}$ & $\mathbf{4}$ & $\mathbf{5}$ & $\mathbf{6}$ & $\mathbf{7}$ & $\mathbf{8}$ & $\mathbf{9}$ & $\mathbf{1 0}$ & $\mathbf{1 1}$ & $\mathbf{1 2}$ \\
\hline $\mathrm{O}$ & 979 & 279 & 117 & 60 & 29 & 34 & 16 & 14 & 10 & 9 & 7 & 3 \\
$\%$ & 62.4 & 17.7 & 7.5 & 3.8 & 1.8 & 2.2 & 1.0 & 0.9 & 0.6 & 0.6 & 0.4 & 0.2 \\
\hline $\mathrm{C}$ & $\mathbf{1 3}$ & $\mathbf{1 7}$ & $\mathbf{1 9}$ & 22 & 24 & 25 & 27 & 29 & 30 & 32 & 3271 posts \\
\hline $\mathrm{O}$ & 4 & 1 & 1 & 1 & 1 & 1 & 1 & 1 & 1 & 1 & 1570 users \\
$\%$ & 0.3 & 0.1 & 0.1 & 0.1 & 0.1 & 0.1 & 0.1 & 0.1 & 0.1 & 0.1 & $\%$ all user \\
\hline
\end{tabular}

Casting a first glance at the corpus, we can learn that the sum total of $3271^{61}$ posts is produced by ca. $1570^{62}$ users - the ratio of posts and users being a first clue to the fact that interaction in this material is not as dialogic, or even polylogic, as one could have imagined. As a matter of fact, there is only a small portion of users who make more than only one appearance within the entire corpus, which reflects the unstableness and fluctuating character, maybe even the enormous size of this special CoP. This latter aspect can, of course, only be determined when investigating the complete content of TSR. According to TSR self-assessment as "the world's largest and fastest growing student community, with over 250,000 members and more than 16,000,000 posts", this estimation seems reasonable. A click on the member list, which is open to public and updated constantly, even discloses the amazing number of more than 460,000 participants. ${ }^{63}$ In figures, the personnel's (inter)activity within this corpus can be described as shown in Table 14.

The pie chart visualizes the high amount of users who contributed only once in all 50 threads of the entire corpus. Indeed, only 56 of all users (3.6\%) authored eight or more posts, with one participant being responsible for the highest amount of posts per user, cpj1987 with 32 posts (see Figure 16).

61. As the length of contributions varies considerably from solitary emoticons to full-grown monologues, it is hard to determine the actual word count for such a corpus. What is more, posts always contain a certain amount of words which are inserted automatically by the system and cannot be counted as part of the actual entry. With this in mind, we can only give an approximation to the sum total of words in this corpus, which amounts to ca. 285,000 words.

62. Note that this figure cannot simply be deduced from the sum total of users per thread, since some users take part in more than one thread. Also, some nicknames are so similar (for example jammydodger09 vs. jammythedoger, katielou vs. katie_lou or Matt_1991 vs. matt_91) that it is questionable whether we are really dealing with two separate users or with one and the same. For that reason, the number of users should only be taken as an approximation.

63. http://www.thestudentroom.co.uk/memberlist.php, accessed September 12th 2010. 


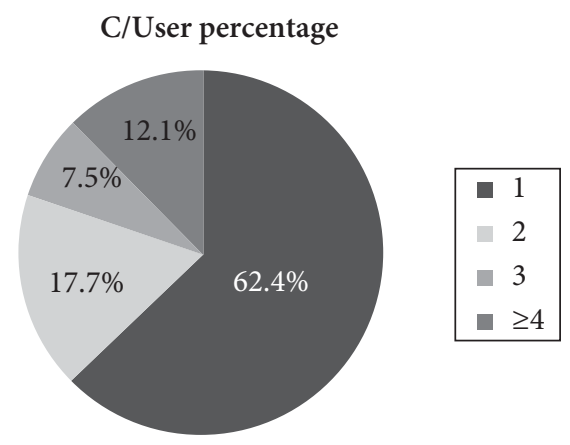

Figure 16. Percentages of contributions per user (C/User)

Obviously, the number of users who could be termed regulars in this corpus is rather slim, since $62.4 \%$ of all users posted only one time, just $2.0 \%$ contributed ten times or more and the small portion of $0.4 \%$ of all users entered the conversation more than twenty times. Based on these figures, I propose the following, certainly arbitrary differentiation of user types (see Table 15). These figures can also be illustrated as a pie chart (see Figure 17), again emphasizing the overwhelming amount of onetime posters.

Since these statistics only give a first, general impression about the users' activities throughout the entire corpus, their significance for users' activities in single threads is certainly limited. The latter, however, is by all means much more interesting and will be focused on in the progress of this chapter.

Table 15. User types according to frequency of contribution

\begin{tabular}{llrr}
\hline User type & & No. & $\%$ \\
\hline onetime poster & $(1$ post $)$ & 979 & 62.4 \\
recurring poster & $(2-19$ posts $)$ & 584 & 37.2 \\
regular poster & $(\geq 20$ posts $)$ & 7 & 0.4 \\
& & $=1570$ & $=100.0$ \\
\hline
\end{tabular}

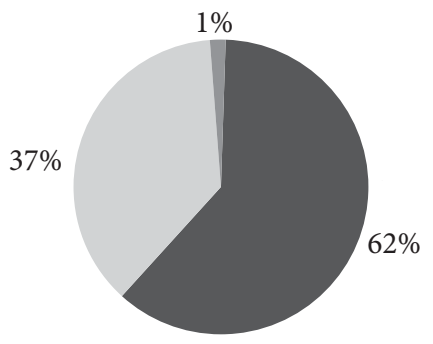

- Onetime poster (1 post)

Recurring poster (2-19 posts)

- Regular poster ( $\geq 20$ posts)

Figure 17. User types according to frequency of contribution (in percentages) 


\subsection{Establishing interpersonal relations online}

To get a picture of interpersonal relations in online message boards, we need to consider what users actually do to establish and/or manage relations and how they proceed in doing so. To begin with, an analysis of the very first stimulus for budding conversations in threads is called for, as subsequent posts are formulated most of the time in reaction to them. This means concentrating on initial posts and their headings. In the first step of the analysis, we will see how topics are introduced and, in so doing, how contact with future interlocutors is established. It will also be interesting to see at a later point whether, on an interpersonal plane, some kind of groundwork for the following course of action is laid at this initial point by the thread starter.

Table 16 proposes one possible grouping of all 50 threads, which was established based on their content and above all their (interpersonal) communicative purpose. It should go without saying that overlaps in categorization can hardly be avoided. Accordingly, the six broad categories (see Table 16) are posited, which will serve as the basis of the second part of this analysis to be conducted (see Section 8.2).

Table 16. Thread titles (headings) according to their communicative purpose

\begin{tabular}{|c|c|c|}
\hline $\begin{array}{l}\text { Communicative } \\
\text { purpose }\end{array}$ & Thread title (headings) & No. \\
\hline $\begin{array}{l}\text { 1. Getting to know } \\
\text { others }\end{array}$ & $\begin{array}{l}\text { \#1 hi all, i’m frankie :) introduce yourselves to me! :) } \\
\# 2 \text { iHola! Soy Lily. }\end{array}$ & 2 \\
\hline $\begin{array}{l}\text { 2. Looking for like- } \\
\text { minded others }\end{array}$ & $\begin{array}{l}\text { \#18 The Official 'I'm going to fail my exams' Society } \\
\text { \#19 Snow Patrol!!!! }\end{array}$ & 2 \\
\hline 3. Asking for advice & $\begin{array}{l}\text { \#11 What does this girl mean by this? (convo included) } \\
\text { \#14 How to stop strange people speaking to me } \\
\text { \#15 To drink or not to drink....? } \\
\text { \#16 What does it mean if a girl smiles at you? } \\
\text { \#24 Would you go on holiday with someone from TSR you don't know irl? } \\
\text { \#34 Pros and cons of boarding school } \\
\text { \#35 Do you use a timetable to study? Or don't you even plan?? } \\
\text { \#39 music /noise whilst staying at uni halls? } \\
\text { \#40 Veganism } \\
\text { \#41 So it seems everyone just wants to ruin there livers in university } \\
\text { \#42 Your pearls of wisdom to university freshers... } \\
\text { \#43 Image change when going to uni? } \\
\text { \#44 Am I wasting my time studying English? } \\
\text { \#45 Best universities for english? } \\
\text { \#47 The best universities for film and tv production? } \\
\text { \#49 Gap Year vs Career...Me vs Parents }\end{array}$ & 16 \\
\hline
\end{tabular}


Table 16. (continued)

\begin{tabular}{|c|c|c|}
\hline $\begin{array}{l}\text { Communicative } \\
\text { purpose }\end{array}$ & Thread title (headings) & No. \\
\hline $\begin{array}{l}\text { 4. Asking for } \\
\text { opinions }\end{array}$ & $\begin{array}{l}\text { \#3 Why Are So Many People On Here Homeschooled? } \\
\text { \#4 booze illegal? } \\
\text { \#6 Are you in favour of Euthanasia? } \\
\text { \#7 Who are more friendly: Northerners or Southerners (England) } \\
\text { \#8 'Life is too short' } \\
\text { \#9 Is TSR an accurate reflection of today's youth? } \\
\text { \#10 Could you ever forget your family? } \\
\text { \#13 Do intellectuals put you off? } \\
\text { \#20 Most underrated Actor? } \\
\text { \#21 Half Blood Prince thread `no spoilers } \\
\text { \#22 Favourite Musical and Why? } \\
\text { \#23 Who's your favourite Friends character? } \\
\text { \#25 What countires have you been to? } \\
\text { \#27 Why modern feminism is illogical, unnecessary, and evil } \\
\text { \#28 Women Make Crappy Musicians } \\
\text { \#29 Creationism of evolution? } \\
\text { \#30 how much of the bible is 'truth' and can god really have made } \\
\text { everything? } \\
\text { \#31 Should governments block websites? } \\
\text { \#32 Are we walking into a surveillance society? } \\
\text { \#33 What makes you happy? } \\
\text { \#37 Grammar schools beat the private sector. } \\
\text { \#46 Can studying literature become too pretentious? } \\
\text { \#50 'Tories plan tougher teacher tests' }\end{array}$ & 23 \\
\hline 5. Blowing off steam & $\begin{array}{l}\text { \#12 I'm bitter and twisted } \\
\text { \#17 Burton's rejected me for a credit card, I think :S } \\
\text { \#26 Has anyone been to America recently ??...border control experience }\end{array}$ & 3 \\
\hline $\begin{array}{l}\text { 6. Offering advice/ } \\
\text { information }\end{array}$ & $\begin{array}{l}\text { \#5 Meteor Shower:) } \\
\text { \#36 Guide to How to Revise Effectively? } \\
\text { \#38 Jolly useful Geography websites } \\
\text { \#48 Q\&A thread - Journalism Courses }\end{array}$ & 4 \\
\hline
\end{tabular}

As Table 16 reveals, the two main communicative purposes of this particular message board are asking others for advice (category three) and for opinions (category four), thus dominating the topical orientation of this cross-section of TSR with 39 of 50 threads.

\subsubsection{What's in a heading?}

As could be anticipated, headings are a way of foreshadowing the content of a post and thus serve as a kind of condensed abstract to it. Accordingly, they exhibit a 
rather compact structure with one to 14 words only, averaging 6.4 words, consisting either of (complex) noun phrases (10 times), (strings of elliptical) interrogative sentences (29 times), or (strings of) declarative sentences (11 times). Naturally, their formal embodiment varies considerably, as it is strongly dependent on users' idiosyncratic ideas thereof. Nevertheless, a lot of thread starters make use of CMC-specific netlingo in including abbreviations, acronyms ("irl", \#24, "Q\&A", $\# 48)$, strings of punctuation marks $(\# 15,19,26)$ and also various ASCII-smileys, which help convey a certain emotional stance that goes with the heading and the post. This can be witnessed in thread \#17, to give but one example. Here, the user expresses their contriteness with the smiley “: $S$ ”. What is more, orthography is respected in most cases as typos hardly occur (for an exception see \#25 and 41). Case sensitivity varies.

With regard to the interpersonal focus of this work, we can discern that headings are of course used to establish a first contact between the thread starter and his potential interlocutors. In order to be successful and to trigger the desired reaction, the thread starter needs to have the purpose of his thread in mind, thus formulating the heading accordingly. This fails only in three cases $(\# 8,40,41)$, in which the headings - voluntarily maybe - are not very revealing at all and do not contain any clues as to what to expect from the thread. All the other headings in this corpus are more goal-oriented. In the first category, Getting to know others, the two users chose basically two strategies: explicitly greeting the audience ("hi all”, \#1, “¡Hola!”, \#2) and introducing their names, thus giving insight into a very small part of their being. Thread \#1 even tried to stimulate the anticipated communication with the rather straightforward yet mitigated imperative "introduce yourselves to me! :)", in which the smiley seems to serve as a softener. All the other headings avoid this type of DIRECTIVE in favor of interrogative clauses, which seem adequate for most of the categories. The interrogative clauses, which appear with (e.g. \#14) rather than without (e.g. \#3) the corresponding question mark, can be very elliptic (\#4 booze illegal?), at times even intertextual and poetic (\#15 To drink or not to drink....?), sometimes consisting of simple noun phrases only (\#20 Most underrated Actor?). In some cases, the question structure is abandoned completely to the benefit of noun phrases (e.g. \#21 Half Blood Prince thread ${ }^{*}$ no spoilers ${ }^{*}$ \#34 Pros and cons of boarding school) or simple declarative clauses (e.g. \#37 Grammar schools beat the private sector.). In view of the categories, users in the third category, one way or another, request advice, while participants of the fourth category try to elicit opinions. Some requests, however, refer to a certain form of the posts to come, as in thread \#21, in which its initiator asks their audience not to post any spoilers (“* no spoilers ${ }^{\star}$ ), at least not in an unmarked way.

The strategy for the second category, Looking for like-minded others, and for the sixth, Offering advice/information, lies in stating the object of the common 
interest, which can either be interesting or helpful for interlocutors, and thus spark off lively discussions. This is achieved by noun phrases, simple and adorned with repetitive punctuation as in thread \#19 (Snow Patrol!!!) or complex and unvarnished as in \#18 (The Official 'I'm going to fail my exams' Society), \#36 (Guide to How to Revise Effectively?), \#38 (Jolly useful Geography websites) and \#48 (QঊA thread - Journalism Courses).

The remaining category, Blowing off steam (number five), is the most heterogeneous one. It can be considered a special case since users do not directly ask for advice or for a discussion but only seem to vent about something, thus sharing their feelings with (unknown) others.

Reactions to these and all the other headings and their corresponding initial posts will show, whether these first, very diverse attempts to establish contact and hence to launch some kind of rapport are considered appropriate.

\subsubsection{The initiators: Investigating thread starters' face claims}

With a look at the thread starters' nicknames, the 50 initial posts were obviously composed by 44 different users, as the user Just Another Student originated a pair of threads $(\# 6,7)$, the user Have Your Say three of them $(\# 31,32,33)$ and four posts $(\# 11,12,14,16)$ appear to have been penned down by Anonymous (anonymous user \#1). While the first two cases are cut and dried, the third one is all the more problematic in terms of consistency of face claims. Although the sparse information given in the profile, only the join date, indicates that we are indeed dealing with one and the same user, the contents of the four initial posts speak a different language: Surely, thread \#11 (What does this girl mean by this? (convo included)) and \#16 (What does it mean if a girl smiles at you?) could have been initiated by the same person, a somewhat inexperienced, insecure male user in need of advice on how to read girls. In these two cases, (unconscious) face claims appear to be consistent. Even the first post of thread \#12 (I'm bitter and twisted) agrees with this impression, as, again, we are presented with a person who suffers from his lack of social skills for the establishment of relations. Fittingly, the incapacity he describes is also mirrored in the very composition of his post, as there is no visible attempt to actually make contact, let alone establish some kind of relation with other users who are, in all likelihood, supposed to help him. His lines provide once more the picture of a withdrawn, asocial character - still we cannot be sure in this case if the post was composed by a male person. Although written under the same nickname, the first post of thread \#14 (How to stop strange people speaking to me) clearly breaks with the aforementioned face claims as, most strikingly, this is the report of a girl ("this 40 year old wolf-whistled at me", "I don't wear revealing clothes or makeup, I'm not pretty”). 
Consequently, we cannot be sure about who is behind these posts, let alone how many persons we are actually dealing with, since neither signatures nor all the other templates provide any further information to convince readers of the authenticity of these posts. Therefore, one might even suspect these posts to be a complete fabrication, strategically placed to inflame a discussion and test reactions. ${ }^{64}$ All of this goes to show that even if communicative platforms such as TSR provide users with a wide range of compensatory mechanisms for the (re)production of social context, they are still not immune from strategic deception such as trolling (see Section 6.2.2) as these mechanisms can easily be circumnavigated.

Fortunately, the other 43 users' face claims are rather low-maintenance, either because they are straightforward and consistent or because there is not much to interpret: While 16 users did not bother to fill out the profile templates at all, 13 thread starters disclosed personal information very selectively. Only 15 users made the most of the options provided by TSR to drop lines and to establish face claims. In addition to personal background information provided by users in their profiles, more clues can be gathered in post-inherent templates, which are either filled out individually by the user or automatically by the system (see Section 2.6.2). Information given in the latter form is certainly more reliable, as users do not have many possibilities to rig these facts. A user's TSR experience thus becomes obvious in the sum total of their posts and in their label as well as in the length of their membership at the point of their posting (shortened LMP), which can be roughly determined through the difference between the time stamp of the post and the date of becoming a member.

Depending on how much personal information is disclosed in the profile, the field of thread starters can be categorized into three broad classes, empty profiles, fragmentary profiles and informative profiles, which contain more or less face claims. The first group of users does not really disclose anything about their personal background. Although we can learn something about their user habits with reference to TSR by looking at the post-inherent templates which are inserted automatically, the individually filled templates are for the most part empty and hence inconclusive: Users mention their name and age very seldom, only giving random hints about their geographic whereabouts - partly via embedding flags,

64. Looked at from a forensic linguistic point of view, arguments can be found which substantiate the claim that the author of all four posts is indeed one and the same person: In contrast to other message board contributions, this user follows case sensitivity consequently, always introduces new segments of his post with paragraphs, displays an almost flawless syntax and orthography and does not make use of typical CMC netlingo. Only the lengths of these posts vary. 
partly explicitly. Accordingly, face claims remain rather vague and can sometimes only be based either on group affiliations, which are traceable via TSR-inherent societies, some telling nicknames or avatars, the latter being used only in one of two cases. With the exception of one user, we are not dealing with newbies who did not find the time to fill out their profiles either but, on the contrary, with more or less experienced users - with memberships ranging from less than a month to ten months tops.

An interesting TSR feature to provide insightful information about users' standing within the CoP, thus revealing previous effort to relational work, is the reputation and warning system (see Section 2.6.2). With eleven users exhibiting an unknown (Anonymous) or small amount of positive reputation - ranging from one (seven times), two (two times) to three (once) gems - we come across two very experienced users with ten and 18 positive evaluations respectively: MelonFace and Lord Hysteria, the latter also being a so called PS Helper. ${ }^{65}$ It will be interesting to see whether this somewhat elevated position among "ordinary" members is also mirrored in their initial posts as well as in their interlocutors' reactions to them. The same holds, of course, also true for the flip side of the coin: Within this first batch of users, there are also three users with negative reputation: Maker (-1), Neo Con (-3) and Don_Scott (-10) seem to have the tendency to stir things up, with the last two even being admonished by moderators repeatedly. This becomes obvious when looking at their warning levels (Don_Scott 3, Neo Con even 5). We will see whether these three live up to their reputation in their first (and subsequent) posts and how other users will respond to them. Finally, it is not surprising either that three of the five users highlighted in this paragraph are in fact veteran users: Neo Con (1295 posts), Don_Scott (3090 posts) and Lord Hysteria (8829 posts).

In contrast to the first of the three groups, most users in the second group give their interlocutors as well as random lurkers a rather good impression about themselves by answering at least to some sections of the profile - the only exceptions being Jambre (\#20) and alienpmk (\#40): Despite filling out one profile section each (school names), this is hardly enough evidence to deduce face claims. All the others, however, detail quite a few facets of their faces, thereby mostly touching on hobbies and interests. Again, we can tell from the societies they are engaged in as well as from nicknames, avatars and various profile sections. In contrast to the previous group, however, some of these users drop clues about their faces

65. A PS Helper is an experienced student and user who takes over responsibility within TSR (see Section 3.3.1) by offering help with the wording of other members' Personal Statements for their university application. As this voluntary work is pursued by a lot of users, we can find 55 PS Helpers among the users in this corpus. 
by means of evaluating themselves explicitly, as, for example, in "Im not a geek" (\#5), "I'm left-wing", "I'm an agnostic" (\#6/7), "I know a thing or two" (\#13), "I am a mature student" (\#28). While most users can be considered established and experienced TSR-members, having composed between 50 and 500 posts, Adonis surely tops the bill with over 6000 contributions within a year. Another veteran user of this group is logic123 with 2218 posts.

In terms of reputation, a slightly different picture presents itself. This time, there are twelve out of 13 users who have a small amount of positive reputation with one (eight times), two (two times) or three (two times) gems and only one user, Adonis, with a rather high amount of reputation (12). Despite and in contrast to these positive evaluations, some users have been warned and even banned by the moderators, such as ShiVji (\#8) and Mr_Mad (\#28), who have 15 warnings and are consequently banned permanently. Two more users, Barz (\#29) and the aforementioned user Adonis have gained two warnings, the latter also being "Banned". With respect to this group, we will thus have to keep a close eye on the behavior of ShiVji, Adonis, Mr_Mad and Barz and on their interlocutors' reactions.

TSR-members of the last group display an intensive, sometimes even playful conduct with most of the sections of their profiles. A large proportion of users posts numerous and extensive self assessments with regard to political views, interests, habits and personal characteristics in general. Consequently, profiles are often exploited as another platform, besides entries in posts, to describe and introduce themselves - a special usage of profiles which will be picked up once more in Section 7.4. Suffice it to say for now that a lot can be deduced from these profiles about their creators' faces and how they want to be apprehended. In some cases, face claims are even contradicting and overtly reveal their contents not be taken too seriously (see thread starter of thread \#3 and Section 6.2.1 for details on the profile of Tha_Black_Shinobi). Above that, even more than half of all users (8 in 15) let an avatar speak for themselves, while the LMP varies considerably from less than a month (including two newbies, superfrankie and lilythrash21, who posted their first contributions the very day they became a member) to several years. The most experienced users are $9 \mathrm{MmBulletz}$ with 1437 posts and Geogger with 3517 posts. Geogger can not only chalk up the highest amount of reputation (12), but is also a PS Helper. Eleven other users also enjoy popularity with one gem (5 times), two gems (2 times), four, five, six and seven gems (each once). While three users do have a negative reputation (-1 for elixira, -2 for J_Hunter and -4 for stellatommo20), it is again a user with positive reputation who has been warned nine times by moderators: $9 \mathrm{MmBulletz}$.

In concluding this section, we can proceed from the assumption that thread starters are a heterogeneous group of users, encompassing newbies as well as 
Table 17. Thread starters as "do-gooders" and "trouble makers"

\begin{tabular}{|c|c|c|c|c|c|}
\hline \multicolumn{3}{|c|}{ “do-gooders" } & \multicolumn{3}{|c|}{ "trouble makers" } \\
\hline nickname & reputation & experience & nickname & reputation & experience \\
\hline $\begin{array}{l}\text { Lord Hysteria } \\
(\# 36)\end{array}$ & $\begin{array}{c}+18 \\
\text { PS Helper }\end{array}$ & 8829 posts & $\begin{array}{l}\text { Don_Scott } \\
(\# 27)\end{array}$ & $\begin{array}{c}-10 \\
\text { WL } 3\end{array}$ & 3090 posts \\
\hline $\begin{array}{l}\text { Geogger } \\
(\# 38)\end{array}$ & $\begin{array}{l}\quad+12 \\
\text { PS Helper }\end{array}$ & 3517 posts & $\begin{array}{l}\text { Neo Con } \\
(\# 17)\end{array}$ & $\begin{array}{c}-3 \\
\text { WL } 5\end{array}$ & 1295 posts \\
\hline $\begin{array}{l}\text { MelonFace } \\
(\# 46)\end{array}$ & +10 & 478 posts & $\begin{array}{l}\text { Mr_Mad } \\
(\# 28)\end{array}$ & $\begin{array}{c}+1 \\
\text { WL } 15\end{array}$ & $\begin{array}{c}19 \text { posts } \\
\text { Permanently banned }\end{array}$ \\
\hline $\begin{array}{l}\text { Sahds } \\
(\# 42)\end{array}$ & +7 & 711 posts & $\begin{array}{l}\text { 9MmBulletz } \\
(\# 34)\end{array}$ & $\begin{array}{c}+1 \\
\text { WL } 9\end{array}$ & 1437 posts \\
\hline $\begin{array}{l}\text { Geritak } \\
(\# 15)\end{array}$ & +6 & 883 posts & $\begin{array}{l}\text { ShiVji } \\
(\# 8) \\
\text { Barz } \\
(\# 29) \\
\text { Adonis } \\
(\# 13)\end{array}$ & $\begin{array}{c}+2 \\
\text { WL } 15 \\
+3 \\
\text { WL } 2 \\
+12 \\
\text { WL } 2\end{array}$ & $\begin{array}{c}\text { ? posts } \\
\text { Permanently banned } \\
420 \text { posts } \\
6349 \text { posts } \\
\text { Banned }\end{array}$ \\
\hline
\end{tabular}

those who have been around for a long time, males (19) as well as females (16) and those who do not want to disclose their sex (9). Primarily, they state to be from Great Britain (30), but also from the United States (2) or from some unbeknownst locations (5). In four cases, other locations were stated; three users gave contradicting and thus unclear information. With users' age varying from 16 to 22 years, with 23 abstentions from disclosing this piece of information, the average age in this cross section of TSR lies at 18.76 years.

In the course of the ensuing investigation, extra attention will be paid to those users who stand out from the other thread starters in terms of experience as well as a heightened amount (i.e. more than five points) of negative and positive reputation, including their warnings levels. In Table 17, these candidates are listed accordingly as either "do-gooders" or "trouble makers".

Although Table 17 displays a surplus on the negative side, this does not mean that users tend to be rather uninhibited. On the contrary, most of these alleged "trouble makers" still possess a positive reputation, which hints at the fact that other users must have considered them valuable interlocutors at some point in the past. This impression is also supported by a look at the overall distribution of positive and negative reputation, which reveals that 38 users have been rated positively by their fellow peers, while only five of them were evaluated negatively (with one case not being reported). The sum total of warnings level among all 44 thread starters is also low with seven instances only. It still goes to show, however, whether this rather positive first impression can be upheld. 


\subsubsection{Face claims in first posts}

A first glimpse of the content of the first posts, which will be examined in some detail in the next chapter, can already be caught here, as it stands to reason to link some of the user related insights gained in the previous section to their first posts. In so doing, I will not only focus on those users who have been highlighted as experienced "do-gooders" and "trouble makers" before, but also cite one or two connections between face claims and first posts of "ordinary" thread starters. A comparison between these two components yields 22 results: While half of the 44 users' face claims are either intangible or cannot be associated at all with any part of the first post, the other 22 cases do exhibit a connection, as one face claim or the other is picked up again somewhere within the post. In a lot of cases (9), face claims, especially those concerning interests, are simply mirrored in the choice of topic for the post:

- Just Another Student $(\# 6,7)$ claims to be interested in debates, thus picking hot topics such as "Are you in favour of Euthanasia?" and "Who are more friendly: Northerners or Southerners (England)";

- i.am.lost (\#9) is a "social media addict", who is very involved in TSR and member of several societies, initiated the thread "Is TSR an accurate reflection of today's youth?";

- Adonis (\#13), who opposes the TSR specific trend to brag with grades and diplomas, asks "Do intellectuals put you off?";

- Geritak (\#15), who is interested in social sciences and enthused about going to university soon, asks herself and others whether drinking could enhance her chances of integration on campus (“To drink or not to drink...??);

- Have Your Say $(\# 31,32,33)$ praises himself as a valuable and smart interlocutor in his label ("Best Thread Starter in Town"), consequently coming up with current and partly controversial topics, which are bound to evoke responses ("Should governments block websites?", "Are we walking into a surveillance society?", but also "What makes you happy?");

- 9MmBulletz (\#34) claims to be "naturally academically intelligent, but [...] also naturally lazy/laid back", prompting him to ask whether boarding schools could be an option to enhance his working morals ("Pros and cons of boarding school");

- stellatommo20 (\#39) is very clear about his musical streak, thus wondering "music /noise whilst staying at uni halls?";

- Eden09 (\#48) exhibits a journalistic streak and consequently opens a "Q\&A thread - Journalism Courses";

- Geogger (\#38), an experienced former student and "Traveller extraordinaire", offers "Jolly useful Geography websites" in her thread. 
A closer look at the last two examples shows that face claims can also be manifest in the choice of purpose of one's thread. This is especially evident in the combination of the category Offering advice/information and users whose lines represent them as experienced, helpful and/or maybe even older than the other users, for which both Eden09's and Geogger's threads are perfect examples. It is thus not surprising that thread initiators in this category are not only valued members but sometimes even PS Helpers, such as Geogger or Lord Hysteria, the latter offering helpful information in his Guide to How to Revise Effectively? (\#36).

Apart from topic and category choices, face claims can also loom in standpoints taken firmly, as in the case of logic123 (\#41, So it seems everyone just wants to ruin there livers in university): This user's lines present him as a straight A student, who proudly discloses his grades, which matches the following statement in his initial post: "Id dont drink alcohol, never will, never have and will not be pressurised into doing so."

What is more, some face claims become evident not only in the choice of content as such but also in the way this content is conveyed: Inexperienced newbies looking for contact, such as alienpmk (\#40) or superfrankie (\#1), are perceived to be very sociable, enthused, even "bubbly", which also shows in their initial entries, which can be colorful (in the true sense of the word) and very straightforward, as proves part of the heading of the first post of thread \#1 "introduce yourselves to me! :)". The "Best Thread Starter in Town", Have Your Say (see above), is indeed rather skillful, since he poses questions without answering them and takes himself out of the focus by quoting articles, thus providing best conditions for a balanced discussion.

The connection between face claims and (the formal side of the) content can also be witnessed in the posts of those users whose lines hint to a rather belligerent background (see "trouble makers" above). More often than not, they do not seem to seek a balanced discussion at all, as the following examples will attest to:

- ShiVji (\#8) seems to be permanently banned (Warnings Level 15) for a reason: The user presents himself very outspoken in his thread "Life is too short", when he teases "If so, why do you come on TSR? It's the most brutal way to waste your 'short life'. Also, is it true that people who live under this philosophy die young?";

- Don_Scott (\#27) not only picks a controversial and polarizing topic but also phrases his view of things unambiguously and unmitigated in the heading Why modern feminism is illogical, unnecessary, and evil. Although this is only the quote of the title of an article, to which he even posts the link, he evaluates it positively, thus underlining his perspective once more: “... great article provides a great answer to the modern feminist myths"; 
- The same holds true for Mr_Mad (\#28): Women Make Crappy Musicians, which is also expressed in the choice of wording. He continues his rant, among others, with "You could probably do some digging on the Internetand if you were a woman you could convince a man to do some digging on the Internet and then", thereby attesting once more to his biased and almost hostile attitude, thus solidifying his negative reputation;

- Neo Con (\#17), posting in the category "Blowing off steam", features a signature in which he quotes and comments on a flame by another user, thereby keeping a former conflict alive. Another hint to his impulsive nature can be seen in his choice of wording ("Wtf").

Counterexamples can be found in the posts of Barz (\#29) and MelonFace (\#46): Despite his negative reputation (Warnings Level 2) and the rather hot topic (Creationism of [sic! or] evolution?), Barz manages to leave a lot of room for discussion:

I personally beleive we have evolved from apes. and that god does not exist. If you beleive otherwise could you state your reasons why and show any evidence to support your claims $\underset{*}{*}$ let the discussions begin [...] plz dont turn this into a flame war.

As the request in the last sentence implies, this user is very aware of the explosive nature of this topic and behaves accordingly. MelonFace lives up to his positive reputation ("do-gooders", see above) by taking every possible measure to avoid friction:

Apologies in advance to any literature students, this is only my opinion. [...] Ok rant over (9) ${ }^{\circ}$, just interested to see if anyone feels the same way? [...] From Past experience I hereby declare to every reader of this thread that: I May Be Completely Wrong And By No Means Endorse Said View Or Expect It To Be Agreed Upon By The General Public And As Such Do Expect Mild/ Severe Criticism So Please Be As Trigger Happy On The Neg Rep As You Wish.

Whether the content and form of this supplement has indeed the desired effect of de-escalation or the exact opposite is yet to be seen. As could be proven in this section, lines cannot only be detected in profiles and other template driven sections within posts. On the contrary, these first impressions can be sensibly linked to and are also graspable in headings and entries in multifarious ways. 


\subsection{Laying the groundwork for thread discussions: First entries}

Much of what has been found out when investigating headings holds also true for first entries - albeit in most cases in a prolonged form. The first entries usually spell out in more detail what has already been insinuated more or less explicitly in the heading. They are the lynchpin for the ongoing conversation insofar as they are the centerpiece of the conversational stimulus, which elicit responses in the form of all subsequent entries. In phrasing answers to these thread starts, which set the standards at least in terms of content, if not also in terms of style and rapport, interlocutors always interpret them on various levels, thus taking them as the yardstick. We should not, however, ignore the fact that first entries do have competition among themselves, as the entirety of comments and responses as well as the relations established therein also serve as a model for subsequent contributions. But let us take one step at a time. First of all, we need to determine what thread starters do in these first entries and how they do it. Only then is it possible to sketch the interplay between first entries and subsequent ones in the proceeding thread or, to be more accurate, to evaluate the interpersonal appropriateness of these first entries based on interlocutors' interpretations and reactions thereof. With this in mind, recurring (interpersonal) patterns of thread starts shall be carved out in the following.

You would think that the first and easiest way of establishing relations consists of greeting your peer group and that those who try to lure others into joining the thread, the thread starters, would greet their future interlocutors. In the realm and reality of TSR, though, greetings are a scarce good. Only seven in 50 posts contain an explicit greeting formula at all, two of which belong to the category Getting to know others, in which a greeting appears to be directly linked to the introduction of self: In thread \#1, the greeting of the heading ("hi all") is followed up with another greeting in the actual entry ("hey"). The same can be witnessed

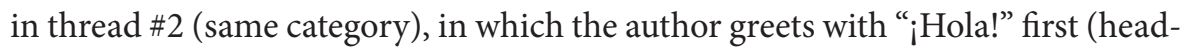
ing), in order to repeat this function in the actual entry with "Hey". Two more greetings form part of first entries in the category Asking for advice: "Well hello there, my first thread." (\#40) and "Hello everyone." (\#42). The other instances of greetings can be found in the category Looking for like-minded others in the first entry of thread \#19 ("Hey"), in the category Offering advice/information in thread \#5 ("Hi guys") and in thread \#48 ("Hi"). Although the small sample is certainly not fit for generalizations, it seems as though first entries only get to see a greeting, when something is at stake, like the acceptance of the two newbie-posters in thread \#1 and 2 in the TSR community, where first impressions count.

Closest to greeting interlocutors is another way of beginning a post, namely by spelling out who the thread is directed at, as in \#8 ('Life is too short'): “The 
thread is aimed at the ones who live life under this philosophy" (my emphasis), thereby also referring back to the heading. Revisiting the heading one way or the other, thus creating lexical cohesion and further topical coherence, is generally one of the most common means of starting (or, for that matter, also ending) an entry, which can be found in almost every thread. Then again, users far and few between also refer to other threads (\#20), link to websites $(\# 6,37)$ or quote parts of or even entire online articles $(\# 27,37)$ to create common ground or preview the form and/or contents of their entry in the first few lines, like in \#13, "Here's a Q." (see also thread \#4 and 11). Especially the latter technique is used quite frequently to pave the way for what is coming up and thus to avoid, as it seems, anticipated misunderstandings and conflicts. The success of this strategy lies again in the eyes of the beholding interlocutors (see Section 8.2). Accordingly, we can find the following metapragmatic utterances:

Now - i'd better say before anyone mocks me - i'm not...

This is not meant to be an overly serious thread btw!

I know it [the TV series Friends] finished years ago, but...,

Now, i dont know if this topic has been brushed apon before but $\mathrm{i}$ wanted to hear peoples opinions as to what they believe.

Apologies in advance to any literature students, this is only my opinion.

(\#46, original emphasis)

Speaking of metapragmatic utterances, they are, of course, not only booked for entry-initial positions but can occur throughout the entire entry, providing as much clarity about the content and users' attitudes towards it as possible, thus trying to avoid tension. To that effect, they are high in number with some entries even making use of metapragmatic utterances more than once:

Allow me to introduce myself.

[...] i feel the need to stress this as [...] - maybe my post is slightly misleading - soz bout that

Here's a Q.

Sorry for the long post.

plz dont turn this into a flame war

Obviously, some metapragmatic utterances are used to comment on the upcoming content in a rather ritualized, fixed form as in thread \#2 and \#13 to give but two examples. Then again others are quite verbose (e.g. \#4, 29), as they detail former reactions in trying to avoid similar experiences. 
In the last and most common way of launching an entry, users start to narrate almost abruptly what has happened to them (to ask for advice subsequently, third category) or what is on their mind concerning a certain controversial issue (to start a discussion, fourth category). The following entry-initial excerpts bear witness to this pattern:

OK, so I'm doing a prjoect over the summer (a Nuffield Bursary) at a place...

I'm 18 and for my entire life, I have been...

Ok so I am paying for my clothes and she goes...

$\stackrel{m}{\odot}$ ok i have gone through all of my education so far in a catholic environment.

Basicaly, Im in first year college and...

Now that we had a look at how thread initial entries are usually introduced, let us jump to the other end and examine how the thread starters at least in this corpus manage to close their very first entries and thus lead over to the interactive part. It certainly does not come as a big surprise that a farraginous variety of closings can be found - even in this small sample - with animated or ASCII smileys playing a central role in a lot of cases, to which the following examples will attest:

Discuss

...might calm your nerves :P

Just a basic traveling question - why not?!

WHAT DO YOU THINK????

Good luck to all sitting exams now and in future!

I'd really appreciate your help.. thanks!!

Help!

Obviously, smileys as keystones of entries can accompany REPRESENTATIVES, EXPRESSIVES and DIRECTIVES (including suggestions to join the group or the discussion), even metapragmatic utterances (\#25). In any case, the composer's emotive stance and attitude towards the content seem to be stressed more or less unambiguously by the presence of this emoticon: While the smiley in \#36 reveals the author's emotional state quite straightforwardly and discloses amusement, it is all the more difficult to tell the smiley's meaning in $\# 4$ beyond a general open and friendly attitude. One could also argue that the smiley serves as a mitigatory device to soften the bare imperative of the DIRECTIVE. In this regard, smileys seem to have a unique way of establishing contact on an interpersonal plane between 
online interlocutors, which is why their impact on further discussions will be closely observed. Sometimes, the closing of entries is even taken over completely by smileys, as they do not directly refer to the previous utterance and are separated from it at least by a paragraph or a break:

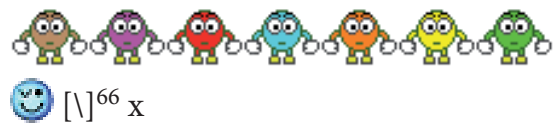

Underneath the smiley in \#23, the small $\mathrm{x}$ indicates a virtual kiss, which can also be found in thread \#40: "Thanks guys. [ 1$]$ x". Although this is quite customary in traditional written exchange between (at least) friends, it seems quite intimate and almost rushed for a message board entry - especially in view of the fact that at that point it is unpredictable who is going to answer at all.

Apart from inserting smileys, there are still other ways of rounding off an entry, among them repeating the initial question(s) $\left(\# 3,16^{67}, 49\right)$, putting in one's own two cents right after initiating a discussion (\#20,25,28) or explicitly asking for people's thoughts and opinions $(\# 6,27,50)$. Naturally, these closings differ considerably from a formal point of view and can be encountered in combination with other closing rituals, such as thanks (\#8, 15, 39, 40,42), links to articles (\#32, $33)$, best wishes and invitations to contact the user $(\# 36,48)$ or again metapragmatic utterances $(\# 29,46)$. Above that, some closings are further ornamented with other accessories such as apologies ${ }^{68}$ like in \#14, "Sorry for the long post.", additional reflections $(\# 6,14)$, general justifications for the existence of the post as such (\#15), even instructions for the moderators as to the form of this thread, e.g. in \#48: "Mods - can this be made a sticky thread?". Finally, some entries do not seem to have a real closing after all, such as threads \#12, 18, 26, 38 or 47, which do not include further closing formulae but simply end with the narration and with a member list respectively. In one case (\#36), the thread starter even made use of a second, subsequent post to add his invitation to discuss his previous contribution.

Although the majority of closings in this corpus does not exhibit any peculiarities, some of them do stand out against the others, such as the following two

66. Borrowed from HTML, the combination $[\backslash]$ will be used henceforth to indicate paragraphs contained in the original entries.

67. Thread \#16 constitutes an exception insofar as the whole entry only comprises one question, which is - to make matters worse - quite repetitive of the heading. Consequently, categories such as beginning or closing of an entry cannot really be applied.

68. The second of the only two apologies to be found within these 50 initial posts is part of thread \#4: “...maybe my post is slightly misleading - soz bout that”, soz being short for 'sorry'. 
DIRECTIVES: While "Discuss." in thread \#13 appeared as totally unmitigated bare imperative, the closing of thread \#21, "Post what you think of the film, or whatever." sounds almost lukewarm and indifferent. What is more, an opinion was presented in the form of an extremely inflammatory statement in \#28, "Women always like to use sexism as an excuse for their lack of genius.", which is most certainly bound to provoke fierce reactions by interlocutors.

The prominent role of smileys has already been stressed in the context of closings. Still they are not confined to that position alone. On the contrary, smileys can be found all over the entry, as they are one major and easy to produce compensatory mechanism for the transportation of emotive prosody. A rather extreme but all the more vivid example of how smileys can be employed to convey an emotional stance presents itself in thread \#1, in which superfrankie, a newbie who seems to be very enthused about being part of TSR now, confides her feelings about the upcoming AS results as follows:

\section{[...] oh dearr! \\ (g) panicpanic!!!! lol} ADORE chocolate, alottt! [...]

Obviously, a variety of (animated) smileys helps this user to express her feelings (sheer panic) quite straightforwardly and unambiguously. In addition to that, she also uses emulated speech in "oh dearr", "aaanyway" and "alottt", capitalization ("ADORE"), acronyms ("lol"), color and font changes and action turns ("panicpanic") as well as repeated punctuation (!!!!) to further substantiate her feelings. Note, however, that this example is the exception rather than the rule, as users usually make moderate use of these compensatory mechanisms and only seldom put the whole array into one single entry. To voice their emotions or at least their attitudes towards a certain proposition, thread starters picked smileys like

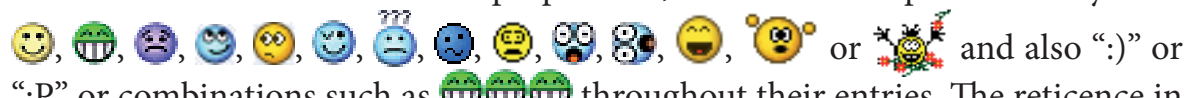
“:P" or combinations such as $\oplus \oplus \mathcal{\Psi}$ throughout their entries. The reticence in view of using compensatory mechanisms and netlingo in general becomes all the more obvious considering the fact that 18 in 50 entries can do completely without any kinds of compensatory mechanisms (\#3,20, 50 among others). What is more, not a whole lot of acronyms and abbreviations has been used either: With the exception of typical CMC acronyms such as lol ('laughing out loud'), rly ('really'), Wtf ('what the fuck'), irl ('in real life') and also kthnxbai ('okay, thanks, bye') and very few other abbreviations like convo ('conversation'), soz ('sorry') and $Q$ ('question'), first entries pretty much resembled regular spoken rather than written exchanges. Accordingly, orthography was partly erroneous and syntax was handled flexibly, especially in terms of punctuation and mixed case, which could also be owed to a certain disinclination to correct typos in this kind of communicative environment. 
Still users fortuitously rather than consciously followed the fifth rule of TSR netiquette and desisted from using much "text speak" (see Section 3.3.2). Whether or not thread starters actually read the code of conduct according to which they are supposed to phrase their contributions, there still seems to be a general awareness about when you run the risk of pushing limits too far. For this reason, Just Another Student, to give an example, starts his initial post in thread \#6 with a prelude about the positioning of his contribution within the categories of TSR (including giving permission to the moderators to move it), which reflects rule number 2 in TSR netiquette ("Post in the correct forum and stay on topic", see Section 3.3.2): "I'm not sure if this should be in the Debate and Discussion Forums or whether it can be in either. I know on this forum you can a broader participation. But feel free to move it, of course." Another thread starter, Anonymous (\#14), is "Sorry for the long post", as this user feels to have overstretched the audience's patience. Then again others, obviously disobeying such rules, are not even aware of doing so: lilythrash 21 starts the second thread with a Spanish heading, clearly violating another part of the fifth rule, which states "Post in the English language unless in an area where it is acceptable to do otherwise (a language society thread or the languages academic help forum.). Outside these areas always provide a translation." Whether these infringements of the TSR code of conduct are actually perceived and commented on and, above all, how interlocutors will handle the first and extremely comprehensive rule "No personal attacks or inflammatory behaviour" is yet to be seen in the second half of this analysis, revolving around hearer reactions.

To round off this chapter, the last few paragraphs will be dedicated to the description of prototypical functional patterns of each of the six broad categories of communicative purposes. Beforehand, we can establish that there are at least two aspects that all of these categories have in common: users' need to explain or even justify the choice and/or genesis of the topic as well as the prominent role of a certain emotional stance, which is contained in almost any post in whatsoever category.

\section{Getting to know others}

Posts of this category mostly contain a lot of personal background information, including the disclosure of current issues of this person's life as well as interests. With the help of simple declarative clauses of the pattern S V Cs as in I'm X. or $\mathrm{S} \mathrm{V}$ O such as $I$ do X., a lot of face claims are revealed. The aim of these kinds of posts, to establish contact with other users, can be pursued quite differently: While superfrankie in thread \# 1 chooses the slightly mitigated but still rather direct imperative "introduce yourselves to me :)" right away (heading), lilythrash21 (thread \#2) resorts to the rather formal and fixed expression "allow me to introduce myself” (entry) to preview her next communicative moves. 


\section{Looking for like-minded others}

As already indicated in the title, users of this category want to find like-minded others in order to find members for a new society within TSR (see Section 2.6.2), dedicated to their mutual hobbyhorse. This activity is per se a social one, aimed at creating interpersonal bonds, for which the target audience is often sketched, the purpose and aim of this society is outlined and existing members are listed.

\section{Asking for advice}

The crucial point in this category is the backdrop of the story, which needs to be narrated and evaluated by the affected person, the thread starter, in order to bring the audience up to speed. Only then is it possible to get advice from the others, the sole purpose of this category, which can be accomplished in many different ways. Looking through the corpus, though, we can detect at least four different types of eliciting advice, perceivably ranging from a low to a high degree of imposition (cf. 4.3.1), with type three and four being approximately on the same level:

1. narration without interrogative clauses explicitly addressing interlocutors;

2. interrogative clauses summarizing the problem without explicitly asking for advice, e.g.: What could she mean by it though? (\#11);

3. explicitly addressing the others when asking for advice, e.g.: What say ye? (\#24);

4. Others: DiReCtives, EXPRESSIVES, REPRESENTATIVES, e.g.: What are the pros and cons and is it for me? [ $\backslash]$ Are you allowed game consoles there and laptops? [two breaks] Please answer as many questions that I pose as you can. (\#34).

\section{Asking for opinions}

The same narrative procedure can be witnessed in this category, which also requires a background to be told first before a discussion can be encouraged - unless, of course, the heading is self-explanatory and already contains the basic issues, as in threads \#29 Creationism of [sic! or] evolution? and \#30 how much of the bible is 'truth' and can god really have made everything? If that is not the case, the bone of contention needs to be spelled out one way or another in the entry. If it is a personal opinion or theory that a user wants to put up for discussion, he needs to make his views known. Otherwise, we are dealing with more or less common knowledge that has to be (re)created in order for it to become common ground. As the following list shows, users in the corpus often proceed by posting (links to) online articles or by quoting or summarizing (parts of) them: 
A very interesting article here: [link] Highlights are: [quotes from the original article]

Here's another story: [link] In condensed form: [summarizing main points]

Thread starters usually avoid imposing on their interlocutors, as they phrase their attempts to create common ground very tentatively, for example with the help of modal auxiliaries or by just posting a link. The decision to visit a website or not is thus left to the interlocutor. Only in some rare cases is the interlocutor faced with excerpts or even with a complete article which he has to read through first before getting the drift of the desired discussion. The second excerpt from thread \#6 obviously does not create common ground per se but rather stresses the necessity of common ground for the upcoming discussion.

To actually launch said discussion, (strings of) interrogative clauses are used to raise the subject. In most cases, these are directly addressed to potential interlocutors:

Also I was wondering why you lot are homeschooled. Did you get bullied at school or have agoraphobia or something? Or do you have really clingy overprotective parents who don't want you to go to school with other teenagers? [...] And also who teaches you? Your parents or do you hire a private tutor(s) to do it

Is the UK sleepwalking into a surveillance state? Do we need more adequate safeguards? Have you been snooped on? (\#32, original emphasis)

Labours argument is that they brand kids as failures? $\stackrel{m}{-}$ But what about the private schools, branding us all as failures in terms of parent income? (\#37)

The second half of this list shows that a few users prefer posing questions impersonally, thus imposing less on interlocutors. In some cases, these questions are followed up with an invitation to discuss, frequently in the form of imperatives, sometimes, however, also as interrogative clauses or as combinations of both:

... - does anyone agree/disagree? [...] Discuss $\because$

Anyway, what are other people's thoughts?

Post what you think of the film, or whatever.

WHAT DO YOU THINK???? $\stackrel{m}{\ominus} \stackrel{m}{\odot}$

Ok rant over (9) ${ }^{\circ}$, just interested to see if anyone feels the same way? (\#46)

Reactions? 
Again, some users do not feel the need to post either a question or an invitation to discuss at all. It will be interesting to see whether there are any differences in the way interlocutors react to these diverse invitations to discuss. Another ingredient which reflects the nature of this category consists of users' evaluations of the topic, viz. adopting one position or the other:

I cant really see any benefits to home education.

...- terrible though it is i can't help but wonder... [...] personally i don't think completely illegalising it is the way to go - perhaps if...

[in answering the question in the heading] No, I am not. [...] My views aren't as strong as they may seem. I am only slightly against it and can see the for arguments.

Women always like to use sexism as an excuse for their lack of genius. (\#28)

I wholly support grammar schools - I think they enable so much social mobility and are a credit to Britain.

While some users mitigate $(\# 4,6,37)$ or even lay low about their opinions, presumably to steer clear from biasing the discussants beforehand, others seem to pursue the exact opposite: In the best case, they are simply open about their point of view (\#3). In the worst case, however, they seem to want to pick a fight (\#28).

\section{Blowing off steam}

The second to last category consists of mainly two parts: the narration of the problem and the evaluation thereof. Compliant with the name of this category, it is not unusual either to show some emotions in this process:

It makes me so angry.

Anyway this is a rant,... [...] I find it so stupid how they cannot say your aplication has been rejected, they always pussyfoot around the subject. (\#17) and whats the reason for your trip??...Lifestory or what..

\section{Offering advice/information}

Finally, examples in this category usually stem from older, experienced users, who feel that they can share their experiences about whatsoever topic with the younger crowd. Accordingly, these thread starts are rather long and can take the form of a guide (\#36), a list (\#38), or a Q\&A section (\#48). These can be accompanied by explanations about the necessity or form of these posts, some also by good luck wishes:

Good luck to all sitting exams now and in future!

Hope this helps. Feel free to ask any more questions $;$ 


\subsection{Other hotbeds for establishing contact: Profiles and signatures}

As a matter of fact, we do not have to look as far as the content of the threads in order to find attempts to make contact with fellow-participants. Two templates to be filled out creatively by users, profiles and signatures, lend themselves for this purpose as well - at least theoretically. As we have seen before (see Section 7.3.2), profiles are seldom filled out and hardly contain any kind of personal information at all. Still, five out of 50 users prove to be the exception to this rule, as they use their profile for this particular purpose, very well knowing that - unlike in thread interaction - the only way to receive a response is via private mail or as part of an entry in one thread or another:

hey! $[\ldots]$

(\#1, profile of superfrankie)

Hiya! [...] Feel free to PM me with queries about any of the above. I don't bite. (\#15, profile of Geritak)

Hi- how are you? No, really. How's the form? [...]

(\#19, profile of imnotapenguin)

[...] Feel free to ask if you want to know more about the course/uni $: ;$ [...] (\#50, profile of lingwist)

An even bigger exception to the rule is DaveSteed24, initiator of thread \#4, and his use of his profile, since he dedicates an entire section of his profile to some of his favorite TSR interlocutors:

(9) you all

There are some people i've met on here who mean an awful lot to me, [ 1$]$ Firstly to Dannidolly who introduced me to the this site i'm really really happy that we've got back into contact $:-$ not having you in my life was really strange and i missed our chats so much $:$ ) here's to many many more $:-$ there are some people i think that you know you're meant to have in your life and thats definitely what i feel with you $: \dot{*}$ i hope so anyway (:) $[\backslash]$ MissAphrodite, talking with you over the past year about everything and anything really means the world to me $:-$ I don't think i could have got through this last year without you to talk to every day $:-$ you always help me with my problems i hope i'm just as good at helping with yours $:$ it would really put a smile on my face to see you happy one day and i definitely am to be able to call you my friend $(-)$ love you sis 3 [।] AccioJellyBean thank you so much for the compliments about my work $: ;$ you gave me faith in it when i needed it and i'll always be grateful - and i hope i'll get to acknowledge you in the back of my book one day $: \vec{*}$ thank you for being my friend and making me smile $:$ you're really one of the 
loveliest, kindest, funniest girls i could ever hoped to have met and uni will be that much more awesome now i'll be going with you $: ;$ [ $\backslash]$ And Writergirl, thank you for always knowing exactly what to say when im down, i don't know what i'd do without you sometimes you really have an ability for making me smile when i dont think i can $:$ you really are one of the sweetest girls in the world $: \dot{i}$ i truly am lucky and grateful that you quoted me on here $:$ i hope you find someone special one day $;$ [ $[\backslash]$ hope i don't get into trouble for writing this

Instead of seeking new contacts by means of his profile, DaveSteed 24 concentrates on existing relationships with fellow-participants. By thanking them for their support and friendship as well as by praising their qualities, he verbally strengthens their positive faces and their need for association as well as his own.

Besides profiles, signatures can also be used as a means of transportation for messages to co-participants, be they known or unknown. Another look at the first 50 posts revealed three such examples, two of which involved thread starters in giving instructions to potential future interlocutors:

Quote me if you want a reply $:-$

(9) $[I]$ Please quote me.
(\#21, signature of $\left.J \_H u n t e r\right)$ (\#48, signature of Eden09)

Exceptional for this category is the signature that Neo Con, originator of thread \#17, has attached to each and every one of his contributions: In displaying excerpts from a bickering with another user, he keeps alive an old flame:

Funny neg rep, nice to see you still thinking of me days on end, who really doesn't have a life hmm

'Funny you mock me for caring too much about TSR when you're spending HOURS each day bickering with your similarly stupid peers. Don't you have anything else to do, I KNOW wanna-be dentists have to study!

I was going to say 'grow some balls' but then again your genome is a cancer on the world, we don't need more weak, stupid, angry and restless retards in this world so I certainly hope you and your trash parents don't excrete out another foul one, we're damn saturated, OK?:ye From DJkG.1'

It further confirms that neoconservatism is a bonafide ideology to protect western civilization and it's values.

Naturally, one can only speculate about Neo Con's reasons for the content of his signature. In terms of facework, however, he clearly distances himself from his interlocutor and threatens his face by parading his interlocutor's aggressive behavior. 


\subsection{Summary: Findings for the establishment of interpersonal relations}

This chapter has given formal and functional insights into integral patterns of thread starts in the form of headings and first entries. As could be shown, headings managed in an average of six words to attract interlocutors' attention for the topic. More often than not, this was insofar successful as the purpose of the thread could be conveyed adequately within this first line (for exceptions see thread \#8, 40, 41), for example by means of greetings and introductory statements in the first category (Getting to know others), stating the target group in the second one (Looking for like-minded others) or by requests for advice and opinions in the third and forth category (Asking for advice, Asking for opinions). Headings were further employed to convey a first emotional stance and included initial signs of mitigation.

The investigation of face claims was insightful in those cases, in which users decided to disclose a certain amount of their personal backgrounds, thus dropping lines for analyzing participants, which could partly be rediscovered either in the form and the content of their first entries. In the course of this, valuable demographic insights about the thread starters could be gained. Above that, a dozen users could be distinguished from the other thread starters based on their extraordinary good or bad reputation, which gives a first reference point as to their previous interpersonal behavior. Accordingly, it will be in the interest of this study to keep a close eye on these "do-gooders" and "trouble makers" in the developing interaction.

Last but not least, the analysis of the first entries illustrated that greetings were a scarce good and rather informal in style ("hey, hi"). Users often decided on other ways of beginning an entry, among them linking to other webpages, quoting (parts of) articles, revisiting the heading or previewing the content, to name but a few. Above that, closings were multifarious and often accompanied by smileys, which is an important feature, among others, to convey emotions and attitudes towards propositions but also to mitigate DIRECTIVEs. More than once, the main part contained a narrative component which set out to create common ground or introduced a personal opinion, subsequently segueing into asking for advice or opinions and invitations to discuss. Finally, metapragmatic utterances appeared to be a welcome means in the attempt to create clarity and avoid ambiguity and could be encountered in various positions within an entry. Whether this attempt is indeed crowned with success will show the analysis of all subsequent entries which found entrance into this corpus.

In the following, it will be interesting to see how interlocutors actually react to what is presented to them, including their verbal behavior with regard to accepting invitations to discuss, answering questions, dealing with and generating 
emotional display, responding to (inflammatory) opinions and much more. Will economics and laziness win over relational work or will it be the other way round, for example when the smoothness of conversation is at stake? In a word, what kind of communicative behavior can we expect from interlocutors? Will they mirror those of thread starters without further ado, will they display completely different kinds of communicative behavior or will they even comment explicitly on what seems either appealing and appropriate or intolerable and inappropriate to them? Although explicit comments will certainly appear only far and few between, we will definitely find implicit comments hidden in the propositions of utterances themselves. Only in the light of hearer reactions is it possible to evaluate and subsequently label thread starters' original posts as appropriate or inappropriate, thus revisiting the comprehensive framework of relational work proposed in Section 5.5 .

A first hint on the judgment of appropriateness of initial posts can already be found in seven of the said initial posts themselves. Even without looking at hearer reactions, we know that the first entries of threads \#4, 6, 30, 37, 39, 44, 46, in their original form, must have caused or at least have been suspected to cause some kind of misunderstanding or tension among the interlocutors. As automatically inserted system notes at the end of each entry reveal, these thread starters saw the need to clarify parts of the entry by modifying its original content - at least once. A look at the time stamps of the posts shows that the users had several minutes to a few hours to alter their contributions after their first publications:

thread \#4 (DaveSteed24):

time stamp original post:

13-08-2009: 13th August 2009 13:37

automatic system note:

Last edited by DaveSteed24 : 13-08-2009 at 16:04.

potential change in content: Prohibition is a stupid idea when applied in real life i feel the need to stress this as a lot of people who have posted seem to think i am pro - maybe my post is slightly misleading - soz bout that

thread \#6 (Just Another Student):

time stamp original post: automatic system note: potential change in content:
10-08-2009: 10th August 2009 23:54

Last edited by Just Another Student : 11-08-2009 at 00:06.

Anyway, what are other people's thoughts? My views aren't as strong as they may seem. I am only slightly against it and can see the for arguments.

thread \#30 (bunny9213): time stamp original post: automatic system note: potential change in content:
18-03-2009: 18th March 2009 13:14

Last edited by bunny9213 : 18-03-2009 at 13:46. 
thread \#37 (Worried Pasta):

time stamp original post:

automatic system note:

potential change in content:

thread \#39 (stellatommo20):

time stamp original post:

automatic system note:

potential change in content:

thread \#44 (elixira):

time stamp original post:

automatic system note:

potential change in content:
09-08-2009: 9th August 2009 19:36

Last edited by Worried Pasta : 09-08-2009 at 19:44.

?

12-08-2009: 12th August 2009 19:40

Last edited by EierVonSatan : 12-08-2009 at 20:51.

?

26-05-2009: 26th May 2009 20:36

Last edited by elixira : 26-05-2009 at 20:40.

P.S. I do not want to be any sort of teacher.

18-06-2009: 18th June 2009 23:13

Last edited by MelonFace : 18-06-2009 at 23:27.

From Past experience I hereby declare to every reader of this thread that: I May Be Completely Wrong And By No Means Endorse Said View Or Expect It To Be Agreed Upon By The General Public And As Such Do Expect Mild/Severe Criticism So Please Be As Trigger Happy On The Neg Rep As You Wish.

As suggested in the excerpts, lurking observers who did not witness the launching of the first draft of the post can only guess as to which parts were altered, deleted and/or added. The passages quoted from the first post of thread \#4 and \#46 are insofar noticeable as they appear to be a supplement to the original, separated from the rest of the entry by a break and standing out for their bold type face and coloring respectively. Also, the P.S. in thread start \#44 most likely seems to be a supplement. The other posts leave much more room for speculation as neither content nor typeface offer any further clues. Another secret revolves around the question whether the change was purely prophylactic or caused by actual reactions. So far we can only be sure about thread \#4, since the user refers to "a lot of people who have posted" which, together with a clarifying statement about this user's point of view in this discussion, must have been the part added later on. The real reason(s) behind all the other modifications can at most only be determined when looking at the subsequent posts. 



\section{Analyzing online message boards II Thread interaction}

\subsection{Introduction}

Seizing up on the description of the fifty first posts gained in the previous chapter, this one undertakes the second step of the analysis by examining reactions to these first posts, as contained in all subsequent posts within the threads, with the management of ensuing interpersonal relations naturally leading the way. Taking the two central concepts from Chapter 4 and 5, facework and relational work, as a springboard, the two major strands of investigation to be pursued in this study can roughly be sketched as in Figure 18. In order to pursue the two strands of investigation, it is necessary to remember and uphold the distinction between the terms relational work and facework proposed in Section 5.3. Although both notions are certainly closely related and share more than one feature (e.g. their discursive nature), they differ in terms of their focal point, which is the negotiation of present and future relationships between individuals in the context of relational work and the negotiation of faces, face claims and basic human needs as subsumed under the notion facework. The scope of latter of the two notions

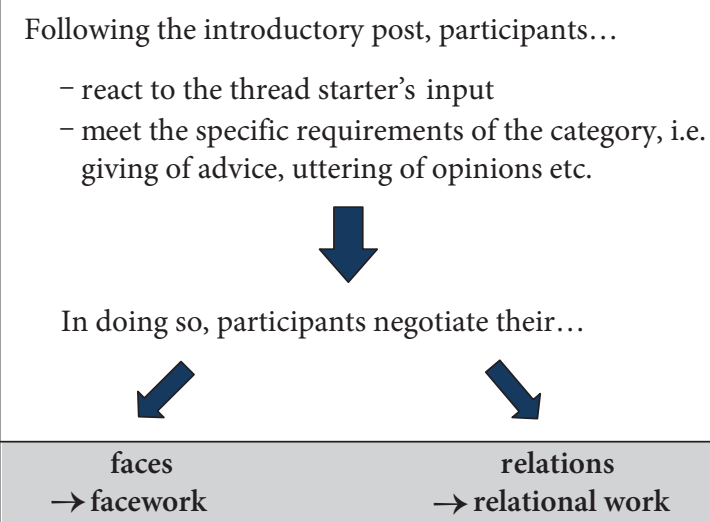

Figure 18. Two strands of investigation: facework and relational work 
also finds expression in the combined model of face as introduced in Section 4.4, which unites the advantages of both Goffman's and Brown/Levinson's notion of face. Based on the understanding of face expressed in this model, we can not only describe the discursive negotiation of face claims among message board participants, but also spot strategies which support or subvert their need for association and/or dissociation within interaction (henceforth positive and negative facework respectively). On the other hand, there is the modified model of relational work (see Section 5.3), which helps us classify the various types of appropriate and inappropriate behavior among interlocutors. According to this schema, all other 3221 posts of the fifty threads, which have not been discussed so far, will be sounded out. While results to the first strand (facework) will be listed qualitatively in the form of strategies for each subchapter, the latter (relational work) will be displayed quantitatively in a grid.

To avoid measuring all threads by the same yardstick and to do their various communicative purposes justice, the analysis will be conducted according to the classification proposed in Section 7.3 (see Table 16), viz. in six groups. To structure the content of all these threads even a little bit further, thus facilitating the actual analysis, their interactional nature needs to be foregrounded: While some threads exhibit a rather flat hierarchy, others are far more complex and branched. For the sake of illustration, Figure 19 shows what two prototypical threads could look like. On the left-hand side, one stimulus, the thread start (indicated by "1"), triggers entries which exclusively respond to the first post, thus creating a low degree of interaction. A high degree of interaction is shown on the right-hand side, where responses refer to the first post (see 2, 4, 6, 8) or comment on the comment of the comment (see, for example, posts 5 and 10). Then again, posts can com-
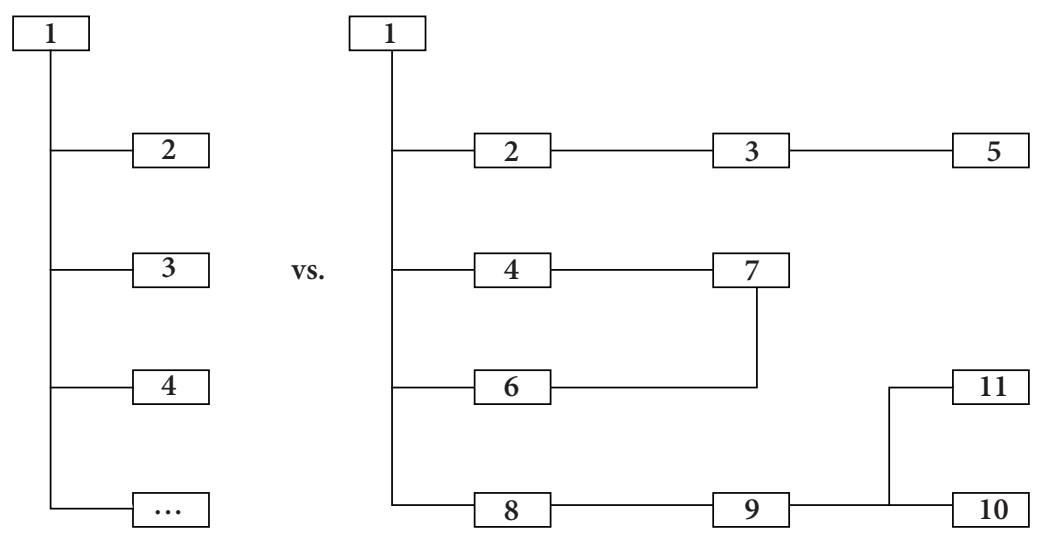

Figure 19. Discourse structure and types of interactivity in two prototypical threads 
ment on the content of two (or more) previous posts simultaneously (see post 7) or on the interaction between two (or more) users (see post 11).

Needless to say that these two types of interaction are prototypical extreme cases, each at an end of an imaginative scale. The more realistic, intermediate types, however, need to be located somewhere in the middle of that scale, displaying at most a tendency to either of the two poles of the scale. If we want to determine the interaction of threads more accurately and distinguish them numerically by that feature, all we need is a simple formula, which interrelates the number of users $(\mathrm{U})$ with the number of posts $(\mathrm{P})$ in a thread, thus generating an approximate interaction quotient: IQ $=1-\mathrm{U}: \mathrm{P}^{69}$ The lowest possible degree of interaction is achieved, when 50 users contribute 50 posts, resulting in an interaction quotient of $0(0 \%)$. Then again, the IQ is the highest possible, when the same amount of posts (50) is created by only two users: $0.96(96 \% \text {. })^{70}$ With the help of this formula, we can group all threads according to their interaction quotient (see Table 18).

A look at the corresponding pie chart (see Figure 20) reveals that the interaction in the TSR corpus is rather low, as almost half of all threads display an IQ below $35 \%$ (48\% of all threads). Then again the small proportion of $14 \%$ of all threads features an IQ above $65 \%$. This results in an average IQ of only $44 \%$ for all threads, which means that only a minority of users in these threads engages in exchanges with more than one turn per interlocutor, i.e. in dialogues or even polylogues. In the best case, we have an IQ of 76\% (threads \#2, 27), at worst only $4 \%$ (thread \#43).

Another look at the two prototypical patterns of interaction described above tells us also something about the nature of the posts themselves. Reconsidering the importance of the hearer perspective for the evaluation of the appropriateness

Table 18. Grouping of the threads according to their interaction quotient (IQ)

\begin{tabular}{lccc}
\hline Interaction quotient (IQ) & No. threads & $\%$ \\
\hline high & $(100-65 \%)$ & 7 & 14 \\
medium & $(64-35 \%)$ & 19 & 38 \\
low & $(34-0 \%)$ & 24 & 48 \\
\hline
\end{tabular}

69. The first part of this formula ( $1-)$ is necessary for the quotient to better mirror the degree of interaction: large numbers for a high degree and small numbers for a low degree of interaction - and not the other way around.

70. The number of one user can be ruled out in this formula, as monologues are not only very unlikely but also uninteresting for this study. For obvious reasons, it is impossible to ever yield an interaction quotient of $100 \%$. 


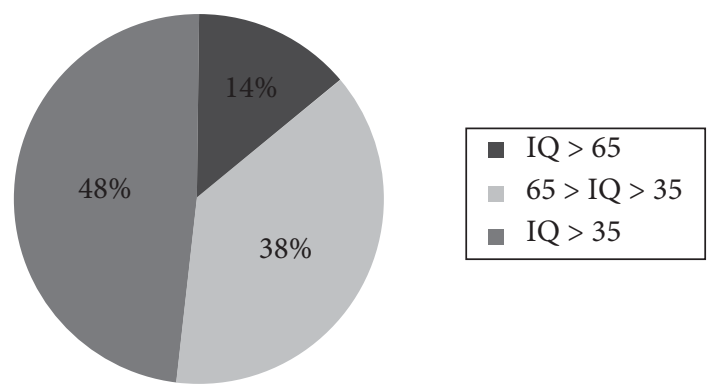

Figure 20. Threads according to their interaction quotient (IQ) in percentages

of posts, we need to develop a system to distinguish those posts which receive value judgments from their successors from those which do not. Ideally these value judgments replace but at least back up the evaluation of the participant observer. Accordingly, we should make a point about three different categories of posts:

- evaluated posts (= type A) are analyzed based on subsequent posts referring to them:

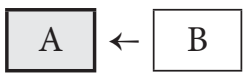

- evaluating posts (= type B) contain value judgments about preceding type A posts:

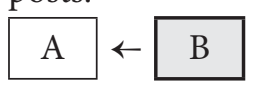

- erratic posts (= type C) stand isolated and without any reference to previous posts: ${ }^{71}$

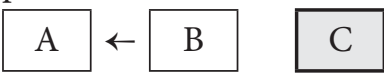

With regard to the communicative purpose of message boards, isolated type C posts are not likely to be found very often in the corpus and will only play a marginal role in this study. Instead, the analysis will focus on the interpersonal appropriateness of type A posts, which can be judged to some degree on the basis of subsequent type $B$ posts. In dialogic structures, however, most posts can be both type A and type B, as they are evaluating (B) and being evaluated (A) at the same time. The only two exceptions to this overlap of functions concerns the first and

71. This also includes posts which comment on the entire thread, lacking reference to one specific contribution previously posted. As potential evaluations contained in this special kind of type $\mathrm{C}$ post cannot be attributed to one or two particular (type A) posts, they are useless for this study. At best, they may be beneficial for capturing one user's impression of the atmosphere of (parts of) the thread. 
the last post within dialogic structures: Lacking a predecessor to evaluate, the first post can only be a type A post. Then again, the last one can only be a type B post, since there is no subsequent post to evaluate it. Consequently, only type A posts in dialogue-initial position and type B posts in dialogue-terminal position (henceforth $\mathrm{B}_{\mathrm{t}}$ ) are functionally fixed.

By their very nature, all 50 initial posts (see Chapter 7) can be labeled type A posts, as they are evaluated by all those subsequent type B posts which refer to the first one. Evidently, these numerous evaluations can vary considerably, in case of which the initial posts concerned will be dealt with separately. The same special attention will, by the way, also be paid for elaborate posts which, for one reason or another, contain more than one direction of relational work evaluating maybe even more than one previous post. Apart from initial posts, a second group of type A posts can be spotted here and there, comprehending those comments to the thread start which serve themselves as springboards for further comments. This is typical for dialogic or even polylogic structures, which are ideal hotbeds for the negotiation of interpersonal relations. Bearing in mind the low IQs discussed above, which hint to flat communicative hierarchies with few dialogic or polylogic structures, it is easily predictable that evaluated, type A posts will be fewer in number than dialogue-terminal type $B_{t}$ posts (see also Table 36 and 37). Since we cannot afford to concentrate on type A posts alone, type $B_{t}$ posts will be an integral part of this analysis as well.

\subsection{Managing interpersonal relations online}

Although the basic course of action and the central concepts for the analysis have been laid out in the introductory section, the first chapter to really dissect the material, 8.2.1, will be quite elaborate in order to demonstrate as clearly as possible the analytical steps and to spell out the underlying methods and assumptions of the results. For that reason, both threads of this category will be discussed in detail. Since qualitative analyses are not only a finicky but also a lengthy business, subjecting each and every one of the fifty threads to this procedure cannot be the aim of this chapter - at least not this graphically. Accordingly, the course of action for the other subchapters will change: Only one thread per category will be examined in detail to be supplemented with insights gained in the other threads subsequently. Noteworthy examples will, however, be quoted whenever necessary.

A first clue to the popularity of topics picked in first posts and/or of the way these were presented by the respective thread starter can be found out rather easily: by simply looking at the speed of interaction. This is revealed in the amount 
of posts per day of each thread (see Table 13, Section 7.2) and can be estimated against the average number of 12.5 posts per day $(\mathrm{p} / \mathrm{d})$.

\subsubsection{Getting to know others}

The purpose of this category, to get to know others on TSR, ideally calls for an interactive exchange between interlocutors. Indeed, both threads in this category, \#1 (hi all, i'm frankie:) introduce yourselves to me! :)) and \#2 (;Hola! Soy Lily.), exhibit a medium to high IQ with $56 \%$ and $76 \%$ respectively. The sub-average number of posts per day, 4.5 and 6 respectively, indicates a moderate interest in the content of both original posts, making them perfectly comparable. Before we can start relating further thread developments to them, the content of the initial posts (henceforth presented in boxes before the analysis) needs to be summarized in the shortest possible way:

hi all, i’m frankie :) introduce yourselves to me! :)

hey, i'm Frankie, i'm 17 and from Nottinghamshire, UK. i’m currently awaiting my AS results which is in 8 days, oh dearr! (9) ${ }^{\circ}$ panicpanic!!!! 101898980 [1]

aaanyway haha.. i love fine art, expressionism, oil paint (just getting covered in paint really lol) and i like Drama too, and Law. (-) i enjoy shopping and long walks on sunny days $\because$ i ADORE chocolate, alottt! [\]

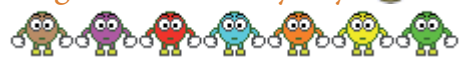

(superfrankie, thread \#1: post 1)

The first entry of thread \#1 was composed by superfrankie (henceforth $s$ ), a newbie who presented herself as a "bubbly", sociable and extremely enthused young girl. These face claims also come out clearly in the way her post is designed (e.g. change of color, exuberant use of smileys and emulated speech, etc.). In trying to get others to introduce themselves to her, she makes her intentions known very soon (heading) and above all quite straightforward (imperative, mitigated with a smiley). To do her bit, she greets her interlocutors before introducing herself.

Before going into any details, it is safe to say that interlocutors' reactions are uniformly positive, as s's behavior is widely accepted and not met with disapproval in any way, thus hinting to an absolutely appropriate way to start a thread. With the exception of some smileys here and there, which are an integral part of every thread, her very idiosyncratic style is neither commented on in any way nor copied. Among the seven users who reacted to her post, four newbies and three established members could be identified, which is insofar important as the kind of response varied according to the respondents' status: Newbies show a tendency 
to recreate (part of) the structure of the introductory post and to pick up and elucidate on the proposed topics (i.e. the disclosure of personal information, AS results). This is quite evident in the following example:

hey $:-$ im lauren, good like with your AS results, i got my standard grade results a week ago. $[\backslash]$ i love drama too, its like my main hobbie $;$

Other newbie reactions saw the creation of very similar posts when compared to the thread starter's attempt to make contact, formally as well as functionally. Still no one, including the thread starter, bothers to answer these two:

Hello all, I am Andre and just become member of thgis community. I really appreciate this TSR lobby and would love to share my views with others.

hey guys, im ali, im waitin on A2 results aswell, good luck 2 u all 4 urs!! :;

Established members' responses, on the contrary, vary considerably in terms of their structure as well as their content and do not copy the initial post. Corresponding to their heightened status, experienced members do not seem to feel the need to concentrate on and introduce themselves or to pick up on presented topics. Instead, they focus on the new user, whom they welcome and include into the group. In some cases, they even praise the value of the new inductee for the community:

$\mathrm{g}_{\mathrm{s}}$ Welcome to TSR. I get my A2 results next week too, not too long now 9 [1] Happy posting

9 [ 1 ] Seems like another great addition to TSR

Big up da Nottingham massive!!”....and all that. welcome $:$

The last user acknowledges the thread starter's home with a casual line about Nottingham before welcoming her also.

The facework towards the thread starter as provided by newbies as well as established members aims at strengthening her sense of belonging and becoming in-group. s's need for association, which is clearly expressed in her post, is thus met. Owed to these very face wants, $s$ gives answers to as many comments as possible (4 out of 7) and does so as suavely as possible, using, for example, first names whenever possible. Thereby, she does not only heighten the IQ of this thread but also initiates several dialogic structures with other users. In so doing, she reciprocates the others' good intentions and fulfills the preset frames of adjacency pairs. In other words, she thanks for welcome and good luck wishes, laughs to indicate 
that she is still present and interested in the conversation or continues conversational topics:

Hi Lauren $:-)$ thankyouu! i'm really nervous... haha. and really? in year 13 we're gonna study Steven Berkoff, should be interesting! how did you do in your results?? $:-$

Thank you for the welcome, Hannah $:$ Good luck in your results too! (p5) hahaha $:$ thankyou very much!! how are you?

Those users who do not disclose much information are also answered, even if only in the same non-committal way:

$\begin{array}{ll}L: & \text { Hi there. I'm new too. } \rightarrow \\ s: & \text { heyyy. how are you? } \because\end{array}$

Since the conversational flow runs smooth and does not exhibit any amplitudes to either side, we can assume that $s$ and her fellow-users consider them at least appropriate, if not even a little bit more. We will return to the question of evaluation a little later.

In this first thread, four dialogic structures can be detected, involving the thread starter $s$ and four other users: $l, h, L$ and $D$. As a matter of fact, two of these four dialogic structures have already been touched on before when talking about adjacency pairs. Accordingly, s's interaction with $h$ basically consists of the thread start, followed by a welcome ( $\mathrm{p} 4$, see above) and a thankful reaction thereto ( $\mathrm{p} 5$, see above). The same holds true for s's short-lived chat with $L$ ( $\mathrm{p} 11,12$, see above). Only two users, $l$ and $D$ do indeed engage in longer conversations with the thread starter, with altogether 7 and 5 turns respectively. $l$, herself a newbie, partly imitates the structure of $s$ 's post in that she also uses a greeting before she introduces herself (see above, p2). Subsequently, she wishes $s$ luck for her exams and picks up on topics introduced by $s$, thereby creating topical cohesion. From an interpersonal point of view, her response establishes common ground between the two, telling $s$ that some of her likes ("drama") and dislikes ("AS results") are indeed shared. Thus having found commonalities to base future interaction on, the following posts revolve around those two topics. More importantly, though, it is not the exchange of information per se but the evaluation thereof which creates closeness and bonding in the form of the positive atmosphere of encouragement and thankfulness on the part of $l$ and $s$ respectively. Concentrating only on the pivotal parts of this interaction, their positive facework surfaces in the following:

s: oh dearr! (90) panicpanic!!!! lol 8989

$l$ : good like with your AS results 
$s:$ thankyouu! i'm really nervous... haha.

$l$ : aww you'l be fine $\because$

s: $\quad$ well done $\because$

l: thanks $\because$

(p7) [referring to l's splendid results]

s: that sounds good!! $\because$

(p9) [referring to l's plans]

Obviously, this exchange is an example of functional and thus appropriate behavior. The well-known question, however, is this: Is $l$ and s's behavior towards each other unmarked or can we detect a surplus somewhere in between their reactions, thus indicating politeness? In a word: Where to draw the line between these two types of interpersonal behavior? A part of the answer to this question certainly lies in the hearer's perspective, which is why it is important to stick to those evaluations that are explicitly made by the interlocutors or that can implicitly be inferred by observing participants on the basis of said subsequent utterances. Unfortunately, this course of action almost never yields concrete labels. If we want to attach them anyway, we need to differentiate between them the best possible way. With regard to message board interaction and following the insights gained in previous chapters (see the working definition of politeness in 5.2 and subsequent elaborations), this study will stick to the following distinction between unmarked/appropriate and positively marked/polite behavior: Any attempt to show consideration for the other (i.e. wishing someone luck, cheering someone on, evaluating something positively or supporting views etc.) will be classified as positively marked/polite behavior, since users are not forced by convention or fear of sanctions to behave that way. This does not, however, include behavior which can be expected by means of the purpose of the category, as for example, advice-giving in an advice thread. There must be a real discernable surplus, originating directly from the voluntary good will of interlocutors. Ritualized reactions thereto, prototypically in the form of thanks, are, however, considered appropriate yet unmarked behavior, because there is actually really not much else for interlocutors to do in order to complete the adjacency pair. Omitting to do so can not only entail negative reactions but even endanger the continuation of the exchange as such.

In illustrating the evaluative processes within $s$ and $l$ 's dialogue and in attaching some labels, the following picture ${ }^{72}$ presents itself:

72. Note that the change of coloring towards the end of s's and l's exchange, from white to grey, represents the types of posts, starting with (white) type A posts in the first six turns and ending with s's (grey) type $\mathrm{B}_{\mathrm{t}}$ post. 


\begin{tabular}{|c|c|c|c|}
\hline$s:$ & $\mathrm{p} 1$ & oh dearr! "(9) panicpanic!!!! lol 89 89 89 & im. $\varnothing$ \\
\hline$l:$ & $\mathrm{p} 2$ & good like with your AS results & ex. + \\
\hline$s:$ & p3 & thankyouu! i’m really nervous... haha. & im. $\varnothing$ \\
\hline$l:$ & p6 & aww you'l be fine $: \dot{\theta}$ & im. + \\
\hline$s:$ & p7 & well done $:$ [referring to l's splendid results] & ex. + \\
\hline$l:$ & p8 & thanks $:$ & im. $\varnothing$ \\
\hline$s:$ & p9 & that sounds good!! $:$ [referring to l's plans] & im. + \\
\hline
\end{tabular}

Once more we realize the importance of spelling things out and reacting verbally (or at least with the help of smileys) in computer-mediated environments (see, for example, p3 and p8), even if this means completing an adjacency pair in the most typical, ritualized way, as mechanisms usually employed in $\mathrm{FtF}$ to respond, like a smile or a nod of the head, are not available in message boards. Still, we should be careful to pigeonhole this behavior as just a case of politeness. Three different types of relational work, which will be picked up again in Table 19, can be discovered in this short excerpt:

- "ex. +" = positively marked, i.e. appropriate, polite behavior as in posts 2 and 7 based on explicit evaluative judgments by the interlocutor (see arrows in posts 3 and 8);

- "im. +" = positively marked, i.e. appropriate, polite behavior as in posts 6 and 9; given the lack of explicit judgments by interlocutors, evaluations remain entirely implicit and can only arise from the participant observer's perspective;

- “im. Ø” = unmarked, appropriate behavior as in posts 1, 3 and 8; again, evaluations remain implicit and draw on the evaluative judgments of the participant observer.

The exchange between $s$ and $l$ eventually ends with $s$ not asking any further questions. This may be due to the fact that she is already engaged in another conversation, this time with $D$. In contrast to the previous dialogue, this one is far more superficial, containing casual small talk and badinages, in which $D$ does not disclose any personal information or pick up any of s's topics:

\begin{tabular}{|l|l|l|l|}
\hline$D:$ & p13 & $\begin{array}{l}\text { Big up da Nottingham massive!!.....and all that. } \\
\text { welcome }:-()\end{array}$ & ex. + \\
\hline$s:$ & p14 & hahaha $:-$ thankyou very much!! how are you? & im. $\varnothing$ \\
\hline
\end{tabular}




\begin{tabular}{|l|l|l|l|}
\hline$D:$ & p15 & $\begin{array}{l}\text { good thanks, enjoyed the nottingham sunshine today } \\
\text { and yourself? }\end{array}$ & im. $\varnothing$ \\
\hline$s:$ & p16 & haha $\because$ i’m goodgood thankyou! you been upto much? X & im. $\varnothing$ \\
\hline
\end{tabular}

What seems to have worked for their conversation so far, comes to a sudden halt: Despite or maybe even because of the virtual kiss, which marks the end of s's last post, her question remains unanswered. While the first three contributions can clearly be labeled "appropriate", we cannot be sure about the last one, since we do not know the reasons for D's decision not to keep their casual chat going. It is more than probable, though, that $D$ did not answer to s's post (16) for other reasons than his feeling of inappropriateness.

In order to count and group the diverse incidences of interpersonal behavior along the lines of unmarked/appropriate, positively marked/appropriate and negatively marked/inappropriate behavior, a matrix will be used to note down the results gained in qualitative analyses. In it, evaluations for type A posts will be distinguished from those of type $B_{t}$ posts. Above that, the matrix also reveals whether judgments of appropriateness were explicitly delivered by interlocutors themselves ("ex.", see above) or whether they could be implicitly read out by the analyzing participant observer ("im."). ${ }^{73}$ Due to the very nature of $B_{t}$ posts, meaning that they can never be explicitly evaluated, the latter distinction only applies to type A posts. With regard to the entire first thread, this is what the matrix looks like (see Table 19).

In evaluating the thread start ( $\mathrm{p} 1)$, the reactions of all seven interlocutors indicate a unanimous judgment of unmarked behavior. The first impression of thread \#1 being an epitome of smooth conversation can now be backed up by figures: no incidence of inappropriate behavior could be found, as all 18 posts were classified as appropriate behavior, eight of which even as positively marked, polite behavior. Four of the politeness labels were directly derived from interlocutors' own verbal estimations, while the other four were the results gained by adopting a participant observer's perspective.

73. Obviously, explicit statements, which reveal interlocutors' personal judgments about the appropriateness of utterances, are more reliable than those of outside observers. Considering the fact that the former are extremely rare, we cannot help but factoring in the latter into our analysis as well. To account at least for the difference of reliability, uninvolved observers' judgments will always be listed separately. 
Table 19. Relational work in thread \#1

\begin{tabular}{|c|c|c|c|c|c|}
\hline \multirow{2}{*}{$\begin{array}{l}\text { Type of } \\
\text { post and } \\
\text { evaluation }\end{array}$} & \multicolumn{3}{|c|}{ Functional $\rightarrow$ appropriate } & \multicolumn{2}{|c|}{ Dysfunctional $\rightarrow$ inappropriate } \\
\hline & $\begin{array}{l}\text { marked + } \\
\text { "polite" }\end{array}$ & $\begin{array}{c}\text { marked + m } \\
\text { "mock-impolite" }\end{array}$ & $\begin{array}{c}\text { unmarked } \\
\emptyset^{74}\end{array}$ & $\begin{array}{l}\text { marked - } \\
\text { "impolite" } \\
\text { ("rude" = r) }\end{array}$ & $\begin{array}{l}\text { marked - m } \\
\text { "mock-polite" }\end{array}$ \\
\hline \multirow{2}{*}{ A: $11 \frac{\mathrm{ex}}{\mathrm{im}}$} & 4 & 0 & & 0 & 0 \\
\hline & 1 & 0 & 6 & 0 & 0 \\
\hline $\mathrm{B}_{\mathrm{t}}: \quad 7 \mathrm{im}$. & 3 & 0 & 4 & 0 & 0 \\
\hline \multirow[t]{2}{*}{$\sum: \quad 18$} & 8 & 0 & 10 & 0 & 0 \\
\hline & \multicolumn{3}{|c|}{18} & \multicolumn{2}{|c|}{0} \\
\hline
\end{tabular}

\section{¡Hola! Soy Lily.}

Hey, I stumbled upon this site last night and found it interesting, so I decided to make an account. [ 1$]$

Allow me to introduce myself. [\]

I'm Lily, and I'm a Cuban-American in Stamford, CT. I'm a musician, a photographer, and a writer. I play bass, guitar, and I sing. [I]

I'm not a very low-profile person. XD [I]

Anyway, I hope to eventually become a part of this community. Can't wait to talk to some of you.

(lilythrash21, thread \#2: post 1)

The initial part of thread \#2 was composed in the same vein as thread \#1, the only difference being the user's method to convey her wish to make contact. Although lilythrash 21 ( $l$ ) also starts her entry with a greeting, which is followed by an introduction of self, she does not force herself on her future interlocutors in the same way superfrankie did: Every so often, she softens the sensed imposition of her addressing others. Evidence for this claim can be found in the metapragmatic yet extremely ritualized utterance "Allow me to introduce myself." as well as in the use of the downtoning adverb "eventually" and the restricting premodification of the noun phrase "some of you".

This post prompted nine other users to react, with four of them being similarly inexperienced as the thread starter herself and five of them being regular experts. As shown by the IQ (76\%), this thread is one of the two most interactive threads in the entire corpus. This is due to the fact that the thread consists of basically five subordinate but at times quite extended dialogues between $l$ and someone else.

74. Note that the combination "ex. $\varnothing$ " does not exist, since explicit evaluations and unmarked behavior are categories which are mutually exclusive. 
The structure alone is thus indicative of the thread starter's wish to embark on conversations that are carried on over more than just two turns. The active part adopted by $l$ is also mirrored in her number of posts: 21 of altogether 42 .

Reactions to the introductory posts are very similar to the ones described in the first thread of this category - the only difference being the fact that new and old users cannot be told apart that easily by their reactions this time. Six out of eight interlocutors start off with a greeting before welcoming her, confirming some of her face claims, declaring her as part of the in-group, picking up on some of her topics (i.e. her Spanish heading and her name), expressing the wish to stay in contact or inviting her to private chats. Some examples:

Hello Lily and Welcome to The Student Room [ $\backslash]$ This place is very addictive! Enjoy your time here!. [I] Eventually become a part of this community.... You already are! $\oplus$

Hello. 9 You sound very talented. $:-[1]$ I hope you stick around. PM me if you need help or a chat.

Hey there $:-[\backslash]$ I'm probably not the best person for advice on this site, I'm kind of new too $\bigcirc$ [ I I just saw your spanish thread title and got a bit excited about it $\oplus[\backslash]$ It's what I want to do at Uni! [ $]$ Anyway, Enjoy the Student Room!

is there anything that your not into? $(1)$ [ welcome to

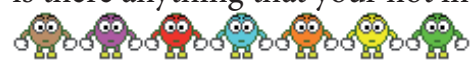

Since she did not explicitly ask her fellow members to introduce themselves, only one user did so voluntarily. ${ }^{75}$ Based on a misunderstanding, one user even tried to give her some technical advice (see below). Further bonding processes involve positive evaluations of things that are dear to the thread starter (i.e. the Spanish language) or shared status ("hi lily, im also new here..^^ and welcome", p28), part of which serve as a basis for dialogic structures.

Again similar to her predecessor, $l$ acts as a moderator within the entire thread and tries to pick up as many conversational strings as possible in order to keep them going. Accordingly, she gives thanks for the kind wishes, offers and advice in English and in Spanish, uses metapragmatic utterances to secure comprehension

75. Note, however, that this user replied in Spanish to reciprocate l's heading (see negotiation of face claims, Section 6.2.1). Maybe one of the simplest phrases to remember in Spanish is an introduction like “¡Hola! ¡Me illamo Simon!”. With regard to his typo and the fact that he later admits to need a dictionary to produce Spanish sentences, one could argue that Simon chose his introduction only as a means to an end and not necessarily with a truly interpersonal intention of introducing himself in mind. 
and avoid friction and positively evaluates ideas brought forward by her comrades. The following excerpts bear witness to this:

Thank you very much. $\oplus$

Why thank you! $\bigoplus$ I just might take you up on that.

Really? Wow, that's kind of nice. $:$

Oh okay. Thanks.

Thank you. And same to you!

$\mathrm{XD}$ gracias.

Now that general types of reactions on both sides have been discussed, let us turn to the inner structure of the dialogic encounters which have not been dealt with so far, focusing again on the relational work. The first rather short dialogue (three turns including the thread start) involves $l$ and $g$ :

\begin{tabular}{|l|l|l|l|}
\hline$g:$ & p15 & $\begin{array}{l}\text { is there anything that your not into? } \\
\text { welcome to } 80\end{array}$ & ex. $+\mathrm{m}$ \\
\hline$l:$ & p17 & Hm... not much that I can think of. XD Thank you. & im. $\varnothing$ \\
\hline
\end{tabular}

P15 exhibits a very interesting form of positively marked, appropriate behavior. Labeled as "ex. + m", g's entry borders on mock-impoliteness, which becomes evident when looking at $g$ 's first smiley. The fact that in her reaction $l$ plays along supports this view. Similar to thread \#1, l's thanks is ritualized and aims at completing the adjacency pair opened by "welcome". It is thus classified as "im. Ø".

\begin{tabular}{|c|c|c|c|}
\hline$R:$ & p2 & 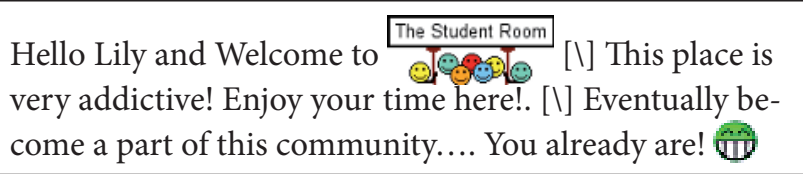 & ex. + \\
\hline$l:$ & p3 & $\begin{array}{l}\text { Thanks! Haha I just meant figuring out how to find my } \\
\text { way around here, you know? }:-\end{array}$ & im. $\varnothing$ \\
\hline$R:$ & $\mathrm{p} 4$ & $\begin{array}{l}\text { Oh right! Well if you need any help then just ask! Anyone } \\
\text { will be glad to help you!. Though Im sure you'l be able to } \\
\text { find you way around. }:-\end{array}$ & ex. + \\
\hline$l:$ & p5 & Thank you very much. & im. $\varnothing$ \\
\hline
\end{tabular}

In this exchange, $R$ almost outdoes himself in offering in-group membership. Funny enough, in his attempt to do positive facework for the newbie, he misunderstands her. In resolving this misunderstanding, $l$ softens the blow by showing 
thankfulness and with the help of emulated laughter at the beginning, downtoning particles ("just", "you know") and a smiley at the end. $R$ accepts her explanations without elaborating on this misunderstanding any further, thus implicitly evaluating her behavior as appropriate. Still he continues his attempts at positive facework in two different ways: by offering help and by judging her as a capable user. This extraordinary amount of consideration is met with thanks by $l$, who thereby accomplishes what is usually expected from her. Although her formulaic expression is adorned with yet another smiley, we should be wary of taking this as an indicator for another type of facework, such as positively marked behavior. After all, almost every entry ends with a smiley.

The exorbitant use of smileys is also evident in the next dialogue, in which $l$ converses with $K$ :

\begin{tabular}{|l|l|l|l|}
\hline$K:$ & p6 & $\begin{array}{l}\text { Hello. } \\
\text { around. PM me if you need help or a chat. }(-)\end{array}$ & ex. + \\
\hline$l:$ & p7 & Why thank you! $\oplus$ I just might take you up on that. & ex. + \\
\hline$K:$ & p8 & Glad to hear. $:-$ I'd be happy to chat. & ex. + \\
\hline$l:$ & p9 & $\because$ & im. $\varnothing$ \\
\hline$K:$ & p10 & $\bullet$ & im. $\varnothing$ \\
\hline
\end{tabular}

After a range of posts which give and reciprocate positive facework verbally, this conversation clearly fizzles out with two posts containing nothing but a regular smiley, whose expressiveness is rather limited, as it only signals something like agreement and joy.

The last of the rather short exchanges to be discussed here takes place between $l$ and $R a$, who picks up on $l$ 's Spanish speaking background by greeting her in Spanish. In so doing, $R a$ makes a grammatical mistake, which he addresses when correcting himself. $l$ attenuates the gravity of his mistake by telling him that she did not even see a need to correct it herself, meaning that she did not consider it worth a face threat:

\begin{tabular}{|l|l|l|l|}
\hline$R a:$ & $\mathrm{p} 39$ & Hola Latino Cien por Ciento! & ex. + \\
\hline$l:$ & $\mathrm{p} 40$ & XD gracias. & im. $\varnothing$ \\
\hline$R a:$ & $\mathrm{p} 41$ & I meant latina of course. $\odot$ & im. $\varnothing$ \\
\hline$l:$ & $\mathrm{p} 42$ & - I wasn't going to correct you, but thank you for that. & im. $\varnothing$ \\
\hline
\end{tabular}


With $D, l$ embarks on a topic-driven conversation, revisiting the central face claim of her initial post, her Spanish background:

\begin{tabular}{|c|c|c|c|}
\hline$D:$ & $\mathrm{p} 11$ & 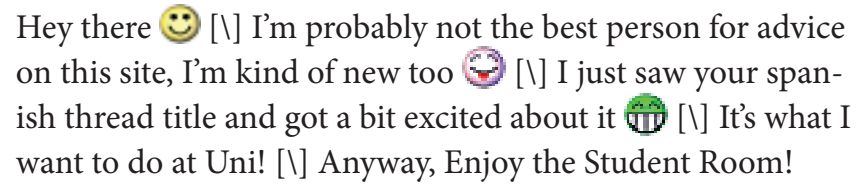 & ex. + \\
\hline l: & p12 & $\begin{array}{l}\text { Oh haha that's cool. Thank you! }:-{ }_{i}[\backslash] \text { I love Spanish, too. } \\
\text { Of course, it's constantly pounded into by my dad and } \\
\text { grandma, but that doesn't change the beauty of the lan- } \\
\text { guage. }[\backslash] \text { Quick question, just out of curiosity. Are there } \\
\text { many Hispanics or Latinos in England? I'm partially of Eu- } \\
\text { ropean heritage, myself, but when I go to Europe, I mostly } \\
\text { stay in Ireland and Spain. }\end{array}$ & im. $\varnothing$ \\
\hline$D:$ & p35 & $\begin{array}{l}\text { Erm... I don't really know. In fairness I can't see how THAT } \\
\text { many would like to move somewhere cold and rainy like it is } \\
\text { most of the time here. [ } \backslash] \text { I presume people will move here, } \\
\text { but I don't think they're the most popular culture to move } \\
\text { here }:-\nabla] \text { I I would love to spend more time in Spain; I re- } \\
\text { ally should try and do some more for my A-level. I feel a bit } \\
\text { of a let down for not doing as much work on it as I thought } \\
\text { I would }(-\end{array}$ & im. $\varnothing$ \\
\hline$l:$ & p36 & $\begin{array}{l}\text { I see what you mean. However, there are a lot of Puerto } \\
\text { Ricans in New York, and it gets considerably cold there. [ } \backslash] \\
\text { Oh, well. I'm sure you were busy with many other things, as } \\
\text { well. Maybe after you complete all these things, you can take } \\
\text { a while to explore the culture in Spain. }\end{array}$ & im. + \\
\hline$D:$ & p37 & $\begin{array}{l}\text { That's true. }[\backslash] \text { I suppose they're probably is a number of } \\
\text { Hispanics living here. I know people that have Spanish rela- } \\
\text { tives tho }:-[\backslash] \text { When I go to Uni I'll hopefully get to go on } \\
\text { the year abroad, which is exciting! [ } \backslash] \text { Have you kept a lot of } \\
\text { your Cuban roots? }\end{array}$ & im. Ø \\
\hline l: & p38 & $\begin{array}{l}\text { That's cool. }:-[\backslash] \text { Oh, yeah. I'd imagine so. I'm actually } \\
\text { about to do the same thing. }:-[\backslash] \text { I do keep my Cuban } \\
\text { roots, and close. I always joke that my dad's house is Cuba } \\
\text { in Connecticut. You step inside, and there are people yelling } \\
\text { in Spanish and making arroz con leche. We have pictures } \\
\text { of Cuba all over the place. We go and visit a lot of family in } \\
\text { Little Havana (AKA, a certain area of Miami) a lot, too. }\end{array}$ & im. + \\
\hline
\end{tabular}


In the first instance, however, $D$ points out that the two of them share the status of newbies, which is why she cannot help her find her way in TSR either - even though she certainly would have liked to offer help. In this way, $D$ has created a common ground between herself and $l$ which is instantly expanded by $D$ 's shared interest for Spanish. Her wishing $l$ a good time in TSR concludes her contribution as well as her positive facework, which is acknowledged positively by $l$. Afterwards, it is $l$ who gives her interlocutor consideration by showing her understanding and cheering $D$ on with her plans, even though $D$ does not explicitly appreciate l's efforts.

The longest of all dialogues in this thread takes place between $l$ and $A$, yet another interlocutor to pick up on her Spanish heading, which is why some part of the conversation is conducted in Spanish, with an English translation provided her and there. The moment this translation is missing, another user, $T$, intervenes to issue a warning: In order for them not to get in trouble with the moderators, TSR netiquette requests entries to be composed in the English language; if they are not, a translation needs to be provided (see Section 3.3.2). Both interlocutors are thankful for the advice, willingly accept this condition and each provide a translation for l's last post. After that, however, their conversation switches back to English. In the end, their exchange is mainly concerned with the weather, a typical subject for small talk and phatic communication, obviously even in computer-mediated environments:

\begin{tabular}{|l|l|l|l|}
\hline A: & $\mathrm{p} 16$ & $\begin{array}{l}\text { ¡Hola! ¡Me illamo Simon! [\] (Hello, my name is Simon). [I] } \\
\text { Welcome to TSR! }\end{array}$ & ex. + \\
\hline$l:$ & $\mathrm{p} 18$ & అ Gracias. & im. $\varnothing$ \\
\hline$A:$ & $\mathrm{p} 19$ & Thats Ok! & im. $\varnothing$ \\
\hline$l:$ & $\mathrm{p} 20$ & $\bigoplus$ & im. $\varnothing$ \\
\hline$A:$ & $\mathrm{p} 21$ & $\begin{array}{l}\text { Ese debe ser el más breve mensaje de siempre. [\] (That must } \\
\text { be the shortest message ever) }\end{array}$ & im. $\varnothing$ \\
\hline$l:$ & $\mathrm{p} 22$ & $\begin{array}{l}\text { Eso es cierto. Probablemente es el más corto. Ahora, ¿qué } \\
\text { hora es allí? }\end{array}$ & im. $\varnothing$ \\
\hline$T:$ & $\mathrm{p} 23$ & $\begin{array}{l}\text { I would provide a translation before the wannabe-mods and } \\
\text { mods arrive. }\end{array}$ & ex. + \\
\hline
\end{tabular}




\begin{tabular}{|c|c|c|c|}
\hline A: & p24 & $\begin{array}{l}\text { Yeh - I'm not that good at spanish - I had to use a diction- } \\
\text { ary to work my answer out. [quote of } l \text { 's p22] = That is true. } \\
\text { It is probably the shortest. Now, what time is it there? [ } I] \text { En } \\
\text { la actualidad unos } 15 \text { minutos hasta el } 4 \text {, y el tiempo ahora } \\
\text { es que no merecen la etiqueta de "verano". [ } I] \text { (It currently } \\
\text { about } 15 \text { minutes until } 4 \text {, and the weather right now isn't } \\
\text { deserving the label of "summer".) }\end{array}$ & im. $\varnothing$ \\
\hline$l:$ & p25 & $\begin{array}{l}\text { Oh okay. Thanks. [ } \backslash] \text { I said, "That is true. It is probably the } \\
\text { shortest. Now, what time is it there?" [ }[] \text { Because here, we're } \\
\text { hours behind. I'm just kind of fuzzy on exactly how many } \\
\text { hours. }\end{array}$ & im. $\varnothing$ \\
\hline$l:$ & p26 & $\begin{array}{l}\text { I'm going to switch back now. }:-(\backslash] \text { Why, is it nice and cold } \\
\text { there? AGH it's hot here. Hate it. When I wrote that mes- } \\
\text { sage before, it was around 9:45 in the morning. Wow... big } \\
\text { difference. }\end{array}$ & $\operatorname{im} . \varnothing$ \\
\hline A: & $\mathrm{p} 27$ & Its not cold, its incredibly humid, and very wet. & im. $\varnothing$ \\
\hline$l:$ & p30 & $\begin{array}{l}\text { What percent humidity? I have friends that live in Texas, } \\
\text { and they HATE how miserable it gets in the summer. }\end{array}$ & im. $\varnothing$ \\
\hline A: & p31 & I have no idea - its alot better today though... & $\operatorname{im} . \varnothing$ \\
\hline$l:$ & p32 & $\begin{array}{l}\text { I hate humidity. It's disgusting. Feels like you're breathing } \\
\text { water. }\end{array}$ & $\operatorname{im} . \varnothing$ \\
\hline A: & p33 & Except that fact that you are not. & im. $\varnothing$ \\
\hline$l:$ & p34 & But you kind of are. Just evaporated water. & im. $\varnothing$ \\
\hline
\end{tabular}

A last word in terms of the evaluation of the very first post of this thread still is order. l's thread start has been directly evaluated by those eight users whose ensuing dialogues with $l$ have been discussed meticulously in the previous paragraphs. All of these eight users concordantly yet implicitly agree on the appropriateness of $\mathrm{p} 1$ ("im. Ø”). With this in mind, the relational work displayed by the ten participants of this second thread is summarized in Table 20.

As the figures attest, the second thread was very similar to the first one as it was also characterized by an extremely harmonious atmosphere. Instances of inappropriate behavior could not be detected and appropriate behavior went mostly unnoticed (29 times). In twelve cases, interlocutors' demeanor could be labeled polite, with one instances of mock-impoliteness as a special type of appropriate behavior standing out in particular.

In summarizing the behavior in the entire first category, Getting to know others, the following recurring strategies could be witnessed, which all added up to 
Table 20. Relational work in thread \#2

\begin{tabular}{l|c|c|c|c|c}
\hline \multirow{2}{*}{$\begin{array}{l}\text { Type of } \\
\text { post and } \\
\text { evaluation }\end{array}$} & \multicolumn{3}{|c|}{ Functional $\rightarrow$ appropriate } & \multicolumn{2}{c}{ Dysfunctional $\rightarrow$ inappropriate } \\
\cline { 2 - 6 } & $\begin{array}{c}\text { marked }+ \\
\text { "polite" }\end{array}$ & $\begin{array}{c}\text { marked }+\mathrm{m} \\
\text { "mock-impolite" }\end{array}$ & $\begin{array}{c}\text { unmarked } \\
\varnothing\end{array}$ & $\begin{array}{c}\text { marked }- \\
\text { "impolite" } \\
\text { ("rude" }=\mathrm{r} \text { ) }\end{array}$ & $\begin{array}{c}\text { marked - m } \\
\text { "mock-polite" }\end{array}$ \\
\hline $\mathrm{A}: 33 \mathrm{ex}$. & 10 & 1 & 21 & 0 & 0 \\
\hline $\mathrm{im}$. & 1 & 0 & 8 & 0 & 0 \\
\hline $\mathrm{B}_{\mathrm{t}}: 9 \mathrm{im}$. & 1 & 0 & 29 & 0 & 0 \\
\hline $5: 42$ & 12 & 1 & & & 0 \\
\hline
\end{tabular}

positive facework on part of the extremely active thread starter as well as his interlocutors. In particular, interlocutors

- greeted each other in various forms

- welcomed newcomers

- offered in-group membership

- exchanged bits and pieces of personal information

- picked up on and elucidated topics introduced by the thread starter, thus creating common ground and topical cohesion

- thanked for considerate utterances

- confirmed face claims

- reciprocated facework by completing adjacency pairs

Maybe due to a lack of experience, newbies showed the tendency towards humbly imitating previous posts in terms of form and content, especially the thread start. Then again savvy, self-confident members did not disclose much information about themselves but rather concentrated on their interlocutors, obviously with the intention to "recruit" as many newcomers as possible into TSR. In that vein, they tried to make them feel welcome and comfortable by instantly including newbies into the community. They also took the liberty of delivering downright positive value judgments about their new fellow-users, which is yet another means of attending to their (explicitly stated) need for association. Although there are no explicit comments about prevalent facework, the lack of interpersonal incidents and the smooth flow of the conversation indicate that everything must have worked within the normal boundaries of conduct within these two TSR-threads. This is once more emphasized by the composition of the sum total of relational work, comprehending 39 cases of unmarked/appropriate behavior and even 21 examples of positively marked behavior (see Table 21 ). 
Table 21. Relational work in the first category (Getting to know others)

\begin{tabular}{l|c|c|c|c|c}
\hline \multirow{2}{*}{$\begin{array}{l}\text { Type of } \\
\text { post and } \\
\text { evaluation }\end{array}$} & \multicolumn{3}{|c|}{ Functional $\rightarrow$ appropriate } & \multicolumn{2}{c}{ Dysfunctional $\rightarrow$ inappropriate } \\
\cline { 2 - 6 } & $\begin{array}{c}\text { marked }+ \\
\text { "polite" }\end{array}$ & $\begin{array}{c}\text { marked }+\mathrm{m} \\
\text { "mock-impolite" }\end{array}$ & $\begin{array}{c}\text { unmarked } \\
\varnothing\end{array}$ & $\begin{array}{c}\text { marked - } \\
\text { "impolite" } \\
\text { ("rude" }=\mathrm{r} \text { ) }\end{array}$ & $\begin{array}{c}\text { marked - m } \\
\text { "mock-polite" }\end{array}$ \\
\hline $\mathrm{A}: 44 \mathrm{ex}$. & 14 & 1 & 27 & 0 & 0 \\
\hline $\mathrm{im}$ & 2 & 0 & 12 & 0 & 0 \\
\hline $\mathrm{B}_{\mathrm{t}}: 16 \mathrm{im}$. & 4 & 0 & 39 & 0 & 0 \\
\hline$\sum: 60$ & 20 & 1 & & & 0 \\
\hline
\end{tabular}

\subsubsection{Looking for like-minded others}

This category brings together users who have something in common outside TSR. In the case of the first thread within this category, The Official 'I'm going to fail my exams' Society (\#18), it is the fear of having failed an exam, while the second one, Snow Patrol!!! (\#19), is designated for fans of this band. Despite this shared orientation, the IQs of the two threads could not differ more with $28 \%$ (low, \#18) vs. $68 \%$ (high, \#19). In terms of posts per day, however, both threads are very similar in sharing the exact same amount of only $0.1 \mathrm{p} / \mathrm{d}$. Since the second one promises more dialogic or even polylogic structures in view of the high IQ, thread \#19 will be chosen as exemplary thread for this category:

\section{Snow Patrol!!!}

Hey I'm thinking of starting up a Snow Patrol Society and was just wondering if anyone would be interested in joining. If you love Snow Patrol then you are very welcome $\oplus \oplus \tilde{\oplus}$

(imnotapenguin, thread \#19, post 1)

Based on common interest in the music group "Snow Patrol", this thread aims at starting a society, thereby inviting fans to join the discussion. The driving force behind this thread is a female user who presents herself as multifaceted, sociable and enthused about the things she likes. Accordingly, she is in search of other users sharing one of her hobby horses. Her thread start does not seem to impose too much on her fellow-users ("I'm thinking of...", "was just wondering if anyone would...”). Then again, her eagerness to attract like-minded users finds expression in her use of repetitive punctuation in the heading, the three smileys at the end of the entry as well as the premodifying adverb very before welcome. 
Among the 23 users who react to this post, only two of them are relatively new to TSR with all the others being experienced TSRians. The comparatively high IQ (68\%) stems from the regular polylogue which is created by the Snow Patrol fans, who do not only engage in dialogic interaction but in-between also comment on the comment. Frequently of the party is the thread starter, who adopts an active role with 18 further contributions dispersed over the thread. Before dialogues do develop it is common practice to signal interest in the society. As the following examples show, the wish to join the society is sometimes adorned with a reason why this user should be allowed to become a member:

meee I adore snow patrol, I listen to their latest album almost daily and I'm still not bored of it. I wish they were coming to Sheffield

Count me in!!!

I wanna join! I wanna join! $\bigoplus\left[\begin{array}{l}\Psi \\ I\end{array}\right.$ I love Snow Patrol

Join me up! Snow Patrol are an incredible band, I've played them 700 times or so in the past 2 months. I think I have an obsession =/ [list of favorite albums]

Apart from expressing the wish to become a part of the society, which enthused users phrase with the help of emulated speech (p8) or repetition of punctuation or entire sentences $(\mathrm{p} 19,32)$, there are other ways for interlocutors to react to the initial post, especially as the conversation proceeds. Naturally with regard to Snow Patrol, participants discuss their opinions about songs and albums, share their experiences with and knowledge about the group and recommend pieces of music to each other. They do so verbally, but also via links and quoted passages from other webpages. Metacommunicatively, some even comment on the thread activity. Here are some examples for posts reacting to the topic rather than to the invitation to join the group:

They ain't what they used to be.

I've just been getting back into them, I keep going through little periods where I listen to loads of them. I liked the second album a lot when it came out, but I keep going back to the first $\oplus$.

Their 1st album was Songs for Polarbears, and the 2nd was When It's All Over We Still Have to Clear Up, are those what you meant?

Honestly my fav band. Ever. $:(1)[1$ Can't believe it's so dead in here tho.

The thread starter adopts an active role in the thread as she welcomes members, comments on and evaluates responses and shares information and emotions: 


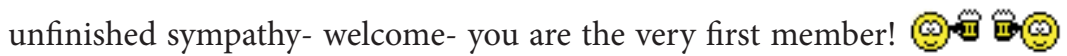

hmmm that is really hard to find. try here: [link] its in dollars like, but they ship worldwide. its pure dear on amazon, something like $£ 20$ for a few tracks! \% $[\backslash]$ You might want to check this out too: [link] however, it's not very clear if it's the 2003 or 2004 version [...]

The following short dialogues involving the thread starter $(i)$ also give evidence for her creating topical cohesion and displaying emotions. In terms of facework, this means the strengthening of interpersonal bonds based on their common topic and by attending to all of their need for association:

\begin{tabular}{|c|c|c|c|}
\hline M: & p32 & I wanna join! I wanna join! $\bigoplus[\backslash]$ I love Snow Patrol & im. $\varnothing$ \\
\hline$i:$ & p33 & $\begin{array}{l}\text { Hey no problem just go here: }[\text { link] }[\backslash] \text { and click Join. }[\backslash] \\
\text { Then just put in your sig that you're a member or something. } \\
\text { Spread the word! There has to be more than } 5 \text { fans here:o }\end{array}$ & $\operatorname{im} . \varnothing$ \\
\hline M: & p34 & 5 members only? & im. $\varnothing$ \\
\hline$i$ : & p35 & $\begin{array}{l}\text { yeah, and that's including me! snow patrol totally rule- there } \\
\text { have to be other sensible like-minded people on this site } \\
\text { surely } \odot\end{array}$ & $\operatorname{im} . \varnothing$ \\
\hline
\end{tabular}

\begin{tabular}{|c|c|c|c|}
\hline$F:$ & p37 & $\begin{array}{l}\text { Join me up! Snow Patrol are an incredible band, I've played } \\
\text { them } 700 \text { times or so in the past } 2 \text { months. I think I have } \\
\text { an obsession }=/[\backslash] \text { What are your favourite albums? My } \\
\text { order of preference goes Final Straw > When It's All Over } \\
\text { We Still Have To Clear Up > Eyes Open > Songs for Polar- } \\
\text { bears (though Velocity Girl, one of my favourite songs is on } \\
\text { that). [ } \backslash] \text { There's not a single track I don't like on any of them } \\
\text { though! }\end{array}$ & $\operatorname{im} . \varnothing$ \\
\hline$W:$ & p38 & $\begin{array}{l}\text { [quote: There's not a single track I don't like on any of them } \\
\text { though!] } \\
\text { Ditto }\end{array}$ & im. Ø \\
\hline$i:$ & p39 & $\begin{array}{l}\text { Hi welcome to the club! haha } 700 \text { times- wow that's dedica- } \\
\text { tion! }[1] \text { Hmm my fav album has got to be Final Straw, } \\
\text { then When It's All Over. I can't choose between the other } 2 \\
\text { yet because im having trouble getting my hands on Songs } \\
\text { for Polar Bears. Nobody sells it!!! } 8\end{array}$ & im. + \\
\hline
\end{tabular}


During the course of interaction, the thread starter reports the official launching of said society, which can be assessed as an effort to expand their commonality, now in the form of a real society on TSR (see 2.6.2). It is also one of three attempts to keep the conversation going, which seem to become more and more outspoken and fervent:

Just to let you know, the Snow Patrol Society is now up and running! $: ;$ [...]

well $\mathrm{i}$ haven't been on in a while but $\mathrm{i}$ just saw the guys in belfast and they were

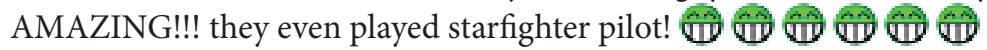

Just thought I'd try to kick this off again! [ 1$]$ Well i must say, i was quite disappointed that the Patrol went home from the Brits empty handed. They totally deserved best album- way better than the Arctic Monkeys 32 38 38 [।] Any one else catch their performance or is planning to go see them soon???

Indeed, her last attempt to reboot the discussion, which seemed necessary to her after half a month of silence, is quite successful: In picking up on a topic which is current and relevant for fans, she sets in motion a very complex polylogue between herself ( $i$ ) and four other members, $F, a, b$, and $D$. Although this thread featured polylogues before, this particular one strikes as impressing due to the interwoven topics and activities of its five authors:

\begin{tabular}{|c|c|c|c|}
\hline$i:$ & p40 & $\begin{array}{l}\text { Just thought I'd try to kick this off again! }[\backslash] \text { Well i must say, } \\
\text { i was quite disappointed that the Patrol went home from the } \\
\text { Brits empty handed. They totally deserved best album- way } \\
\text { better than the Arctic Monkeys } 35[\backslash] \text { Any one else } \\
\text { catch their performance or is planning to go see them soon??? }\end{array}$ & $\begin{array}{l}\text { im. } \varnothing \\
(41, \\
46, \\
47)\end{array}$ \\
\hline$F:$ & $\mathrm{p} 41$ & $\begin{array}{l}\text { Yeah, I was disappointed too } 8[\backslash] \text { Performance?! Where? } \\
\text { When? } \because \text { *hopes it is near-ish* }[\backslash] \text { Do you know how hard it } \\
\text { is to get hold of this shirt? Very hard, take my word for it } \\
\text { Been trying for while now - so far, one American company } \\
\text { that never sent it, and one off eBay that's a bit too tight } \odot \text { I'll } \\
\text { get it eventually... }[\backslash]-\text { Forgotmytea }\end{array}$ & im. $\varnothing$ \\
\hline
\end{tabular}




\begin{tabular}{|c|c|c|c|}
\hline$i:$ & p42 & $\begin{array}{l}\text { Unfortunately, i meant their performance on TV-if only they } \\
\text { would hurry up and play the UK again!!! [\] That's a nice shirt } \\
\text { I must say- do they sell it on the shop section of the official } \\
\text { site? I've been looking for one myself that has the wee snow- } \\
\text { flake logo on it- feel free to send me the one that didn't fit! } \oplus \\
{[\backslash] \text { I've just noticed the bonus points available for guessing }} \\
\text { the deep obscure meaning behind your location and i think i } \\
\text { know the answer. Do I get a prize??? }\end{array}$ & $\operatorname{im} . \varnothing$ \\
\hline$F:$ & p43 & $\begin{array}{l}\text {;no; Sadly, they only have it in green on their site, which is (in } \\
\text { my opinion) nowhere near as nice When are they playing } \\
\text { on TV, then? I don't have aTV Here at uni so I'll have to } \\
\text { email home and get my parents/brother to record them } \\
\text { [ }] \text { Well, if by bonus points you mean rep, then yeah } 11] \\
\text {-Forgotmytea }\end{array}$ & im. Ø \\
\hline$F:$ & p44 & $\begin{array}{l}\text { [announcement from Snow Patrol webpage] [\] Sounds } \\
\text { good - I might just have to go to that.... Anyone else } \\
\text { planning on seeing the Patrol live then? }[\backslash] \text {-Forgotmytea }\end{array}$ & $\operatorname{im} . \varnothing$ \\
\hline$i:$ & p45 & $\begin{array}{l}\wedge \wedge \wedge \text { oh wow nice one!!! I’m definitely going to do my best to } \\
\text { get to that! It'll make up for not going to Oxegen } \oplus\end{array}$ & im. Ø \\
\hline$a:$ & $\begin{array}{l}\mathrm{p} 46 \\
(\rightarrow 40)\end{array}$ & $\begin{array}{l}\text { Hey everyone, I love Snow Patrol they are one of the best } \\
\text { bands in a long while. Have seen them twice in Northern } \\
\text { Ireland and can't wait to see them headlining at Oxegen, it } \\
\text { should be great. I agree about the Brits they were robbed. }\end{array}$ & $\begin{array}{l}\operatorname{im} . \varnothing \\
(48, \\
49)\end{array}$ \\
\hline$F:$ & p48 & 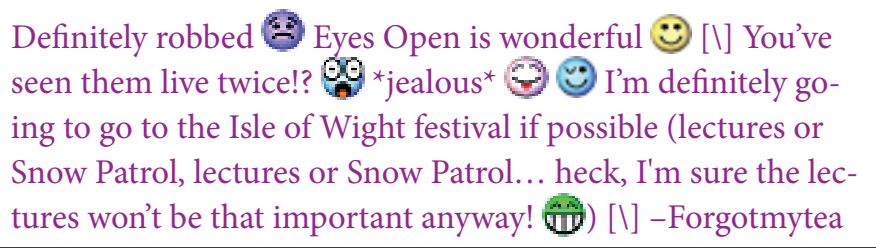 & $\operatorname{im} . \varnothing$ \\
\hline$i:$ & p50 & $\begin{array}{l}\text { ooooh tough choice there- just get someone to tape the lec- } \\
\text { tures for you- sorted! }\end{array}$ & $\operatorname{im} . \varnothing$ \\
\hline$b:$ & $\begin{array}{l}\mathrm{p} 47 \\
(\rightarrow 40)\end{array}$ & $\begin{array}{l}\text { No I didn't see it but I defo think that Snow Patrol should } \\
\text { have got the best album - although I do love the Arctic Mon- } \\
\text { keys too. }\end{array}$ & im. $\varnothing$ \\
\hline$i:$ & $\begin{array}{l}\mathrm{p} 49 \\
(\rightarrow 46 \\
47)\end{array}$ & 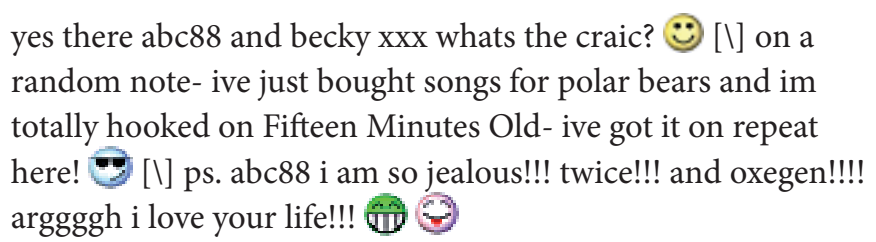 & $\begin{array}{l}\operatorname{im} . \varnothing \\
(51, \\
54)\end{array}$ \\
\hline
\end{tabular}




\begin{tabular}{|c|c|c|c|}
\hline$F:$ & p51 & 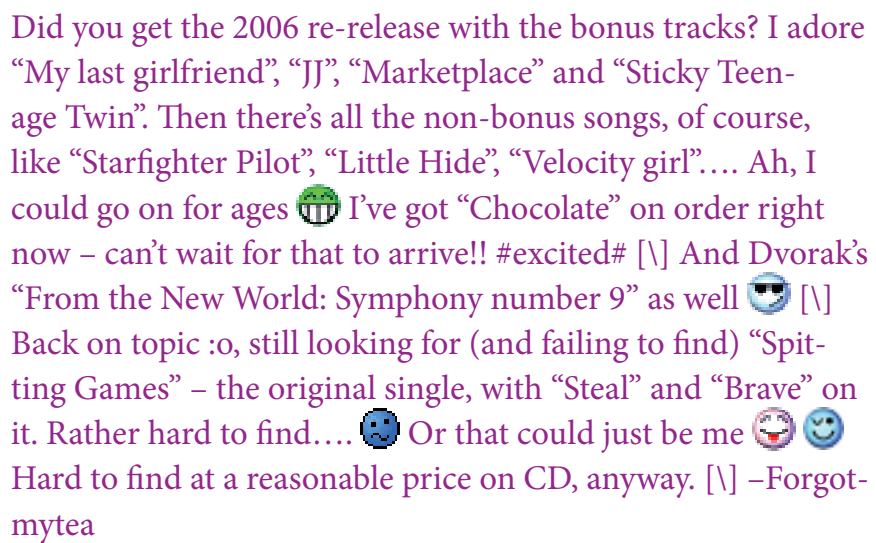 & im. $\varnothing$ \\
\hline$i:$ & p52 & $\begin{array}{l}\text { hmmm that is really hard to find. try here: [link] [ } \backslash] \text { its in dol- } \\
\text { lars like, but they ship worldwide. its pure dear on amazon, } \\
\text { something like } £ 20 \text { for a few tracks! } \$ 9 \text { [ } \backslash] \text { You might want } \\
\text { to check this out too: [link] [ } \backslash] \text { however, it's not very clear if } \\
\text { it's the } 2003 \text { or } 2004 \text { version }[\backslash] \text { If I find anything better Ill let } \\
\text { you know! Oh, and during my searches I found that shirt you } \\
\text { were looking for: [link] [\] Yeah I got the } 2006 \text { release, so i } \\
\text { have piles of new tracks that i haven't got round to listening to } \\
\text { yet. I still have a couple to hear on When It's All Over... cause } \\
\text { I keep on replaying Olive Grove and cant get past it! :o }\end{array}$ & ex. + \\
\hline$F:$ & p53 & 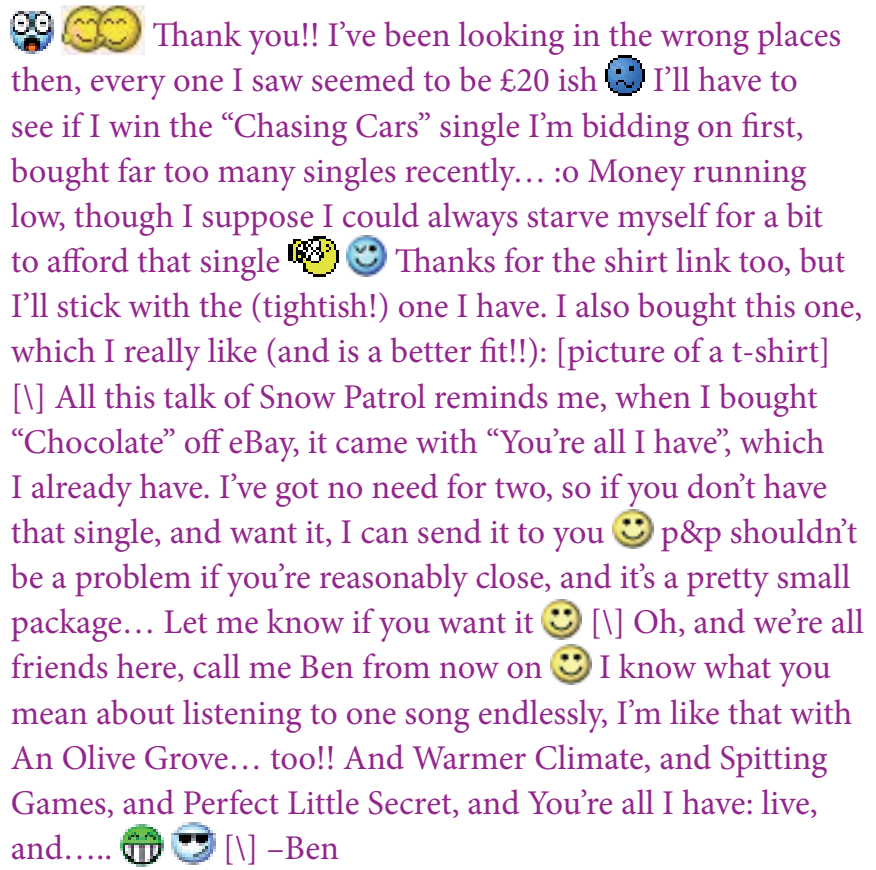 & $\begin{array}{l}\text { im. } \varnothing \\
(61) \\
\text { ex. }+ \\
(56)\end{array}$ \\
\hline
\end{tabular}




\begin{tabular}{|c|c|c|c|}
\hline$D:$ & p61 & $\begin{array}{l}\text { I've got this t-shirt [link] }[\backslash] \text { Well, nearly that t-shirt. I bought } \\
\text { it at a gig they did at Meadowbank Stadium in Edinburgh last } \\
\text { year (octoberish I think). Fits really well and I luuuuve it lol. } \\
{[\backslash] \text { I really want to go to see SP again but im starting uni in }} \\
\text { september so wont have time for a while } 8\end{array}$ & im. Ø \\
\hline$F:$ & p62 & 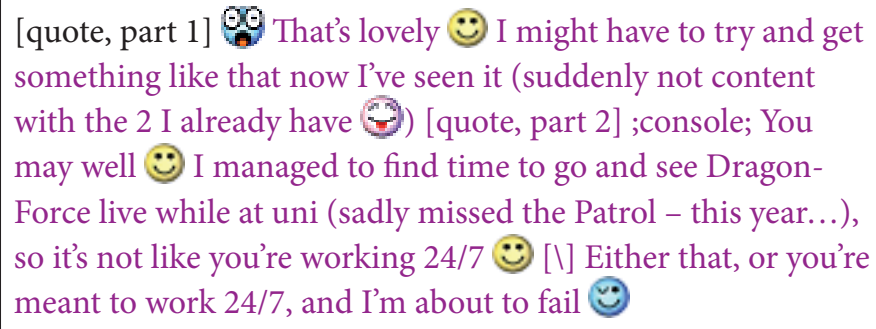 & im. + \\
\hline$D:$ & p64 & $\begin{array}{l}\text { Well apart from Radio 1s Big weekend I've not been to see } \\
\text { any other bands like that. Fantastic feeling and at the Ed- } \\
\text { inburgh one I was right at the front to the left of the stage. } \\
\text { Gives me shivers just thinking about it (apart from when a } \\
\text { drunk old man spilled his beer on my ankles lol, that's a dif- } \\
\text { ferent kind of shiver). I think that t-shirt cost me } £ 18 \text { - most } \\
\text { expensive t-shirt I've ever had lmao but it's lovely really. [ } \backslash] \text { As } \\
\text { I say, that one's slightly different but the picture's the same. On } \\
\text { the back it had the tour-dates on it too. [ } \backslash] \text { Anyone else loving } \\
\text { "Open Your Eyes"? Really love the bit near the end where it's } \\
\text { just crashing music - very overwhelming eh? }\end{array}$ & im. Ø \\
\hline$i$ : & $\begin{array}{l}\mathrm{p} 56 \\
(\rightarrow 53)\end{array}$ & $\begin{array}{l}\text { [quote, part 1] Im glad i was some help! yeah i spend wayyyy } \\
\text { too much on CD's, as soon as I get paid im off to HMV } \oplus \\
\text { [quote, part 2] Wow thats really nice of you! } \because \text { I'll have to } \\
\text { have a hunt around my room to see if I have that one and I'll } \\
\text { let you know- might take a while though, my room is such a } \\
\text { mess :o }\end{array}$ & im. + \\
\hline$a:$ & $\begin{array}{l}\text { p54 } \\
(\rightarrow 49)\end{array}$ & $\begin{array}{l}\text { Thanks lol. If it is any consolation the second time I saw Snow } \\
\text { Patrol was at a festival in Northern Ireland and had to spend } \\
\text { most of the set looking for my friend who i had lost. They are } \\
\text { great live though especially the first time I saw them at the } \\
\text { Ulster Hall. When they played 'Run' everyone got out their } \\
\text { lighters and mobile phones and started singing. [ } I] \text { I am really } \\
\text { loving Eyes Open at the moment so I can't wait to hear them } \\
\text { perform songs from the album. }\end{array}$ & $\begin{array}{l}\operatorname{im} . \varnothing \\
(55, \\
57)\end{array}$ \\
\hline
\end{tabular}




\begin{tabular}{|c|c|c|c|}
\hline$F:$ & p55 & 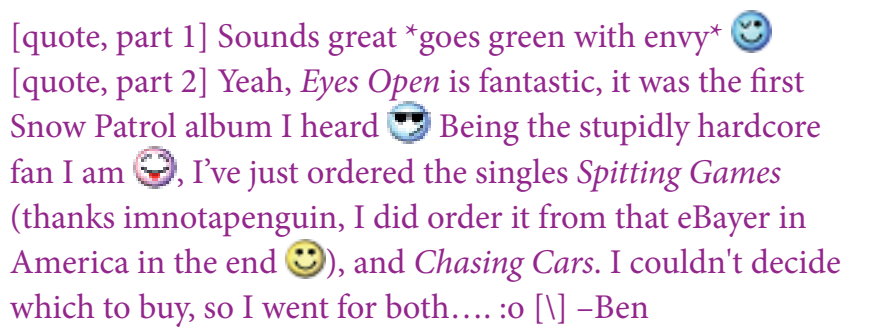 & $\operatorname{im} . \varnothing$ \\
\hline$i:$ & $\begin{array}{l}\text { p57 } \\
(\rightarrow 54)\end{array}$ & $\begin{array}{l}\text { aw I would love to see them in the Ulster Hall- it would be } \\
\text { nice to see them in a smaller venue than the Odyseey, because } \\
\text { they are closer to the audience and its just a more intimite } \\
\text { gig- you feel like you are their best mate for a couple of hours! } \\
\text { I went to see Duke Special in a small club, after hearing them } \\
\text { support the Patrol in december, and he was brilliant- highly } \\
\text { recommended. }\end{array}$ & $\operatorname{im} . \varnothing$ \\
\hline$a:$ & p58 & $\begin{array}{l}\text { Yeah I agree, I love the Ulster Hall, you feel so close to the } \\
\text { band on stage. I love Duke Special!!!! i am soo jealous that } \\
\text { you got to see them. Hopefully I will see the at oxegen as i'd } \\
\text { say they are good live. }\end{array}$ & $\operatorname{im} . \varnothing$ \\
\hline$i:$ & p59 & $\begin{array}{l}\text { aye they were brilliant- although it was so typical right- me } \\
\text { and my friends managed to get right to the front, and then } \\
\text { near the end they come down off the stage, go to the back of } \\
\text { the hall, and start singing there! All the people at the back } \\
\text { had a perfect view and we couldn't see a thing! } \\
\text { they going to be at Oxegen? ohhhh i am so ragin i didnt get a } \\
\text { ticket!!! Although im not sure the whole tent thing would be } \\
\text { my scene- id have to check into a nearby 5-star hotel and only } \\
\text { emerge on the field when the patrol appear! } \oplus\end{array}$ & $\operatorname{im} . \varnothing$ \\
\hline$a:$ & p60 & $\begin{array}{l}\text { Duke Special are not on the bill for oxegen. But I am hoping } \\
\text { they will be added later as I really want to see them play live. }\end{array}$ & $\operatorname{im} . \varnothing$ \\
\hline
\end{tabular}

Obviously, this polylogue does not provide any exceptional cases regarding relational work, as most entries are considered to be perfectly appropriate, with only four exceptions showing a tendency to positively marked, polite behavior. Owing to the polylogic structure, however, five entries (p40,46, 49, 53, 54) received more than one evaluation, viz. two or even three (p40). Although this has no consequence for four of these five posts, in which evaluations are unanimous, the fifth example (p53) yielded diverging results: p61 did not judge it at all (“im. Ø”), p56 explicitly praised its author ("ex. +"). There is, however, a compelling reason for this: While the author of p56 was offered a kind gift in p53, for which she is 
190 (In)Appropriate Online Behavior

Table 22. Relational work in thread \#19

\begin{tabular}{|c|c|c|c|c|c|}
\hline \multirow{2}{*}{$\begin{array}{l}\text { Type of } \\
\text { post and } \\
\text { evaluation }\end{array}$} & \multicolumn{3}{|c|}{ Functional $\rightarrow$ appropriate } & \multicolumn{2}{|c|}{ Dysfunctional $\rightarrow$ inappropriate } \\
\hline & $\begin{array}{l}\text { marked + } \\
\text { "polite" }\end{array}$ & $\begin{array}{c}\text { marked + m } \\
\text { "mock-impolite" }\end{array}$ & $\begin{array}{c}\text { unmarked } \\
\varnothing\end{array}$ & $\begin{array}{l}\text { marked - } \\
\text { "impolite" } \\
\text { ("rude" = r) }\end{array}$ & $\begin{array}{l}\text { marked - m } \\
\text { "mock-polite" }\end{array}$ \\
\hline \multirow{2}{*}{ A: $44^{*} \frac{\mathrm{ex} .}{\mathrm{im}}$} & 2 & 0 & & 0 & 0 \\
\hline & 1 & 0 & 41 & 0 & 0 \\
\hline $\mathrm{B}_{\mathrm{t}}: 30 \mathrm{im}$. & 3 & 0 & 27 & 0 & 0 \\
\hline \multirow[t]{2}{*}{$\sum: \quad 74$} & 6 & 0 & 68 & 0 & 0 \\
\hline & \multicolumn{3}{|c|}{74} & \multicolumn{2}{|c|}{0} \\
\hline
\end{tabular}

* +1 exception p53: im. Ø, ex. +

thankful, this offer did not concern the user who composed p61, who commented on other parts of the rather longish contribution of p53. As an exception with more than one evaluation, this entry cannot become part of the evaluative matrix, but needs to be treated separately.

The impression of a harmonious and smooth conversation among like-minded users is further substantiated by other dialogic as well as polylogic structures, in which appropriate, unmarked behavior clearly prevails. This also includes the thread start, which was implicitly but nevertheless unanimously considered to be appropriate (“im. Ø”) by twenty users. The results gained for this thread are summed up in Table 22.

The Official 'I'm going to fail my exams' Society

Clearly a lot of people are going to faill their exams. (1). Only joking 6 . [ $[1]$ No successful people are allowed on this thread - only people who are going to fail or who feel miserable about their exam performance. You can all cry on this thread without being riduculed by succesful people. [ I] [list of 27 members]

(inequality, thread \#18, post 1)

The sole purpose of the second thread in this category is to comfort those who believe to fail or have failed an exam. For this reason, the thread starter makes it perfectly clear that "successful people" are excluded. In contrast to thread \#19, some obvious differences need to be addressed. First of all, thread \#18 contains the first type $\mathrm{C}$ posts so far, the relevance of which will be discussed later on. What is more, the hierarchy of the communication structure is extremely flat: dialogues, let alone polylogues are a scarce good, a fact which is also mirrored in the distribution of the types of posts (see Table 23). Most entries refer to and answer the 
initial one, which they implicitly evaluate as unmarked, appropriate (“im. Ø”). Fittingly, the thread starter, $i$, plays an extremely subordinate role: In contributing only two further posts, he leaves the thread largely unmoderated. In his first post after the initial one, $i$ tries to silence a trouble maker who aims at bringing him into disrepute by telling his fellow users that $i$ is a fraud:

\begin{tabular}{|l|l|l|l|}
\hline$a:$ & $\mathrm{p} 4$ & $\begin{array}{l}\text { this person is so cool hes going to cambridge hes doing } \\
\text { double maths and he got 100\% in all the maths exams so } \\
\text { far P1-6 M1-3 S1 }\end{array}$ & ex. - r \\
\hline$i:$ & $\mathrm{p} 5$ & Ne [sic! Be] quiet, go and work in wyevale. & im. - r \\
\hline$a:$ & $\mathrm{p} 47$ & $\begin{array}{l}\text { inequality has already got an A in maths and hes do- } \\
\text { ing double maths he was doing 5 A levels, double maths, } \\
\text { chem, phys and music but he dropped chem after AS then } \\
\text { he dropped music aabout 2 months ago and he doesn't } \\
\text { even go to maths he teaches himself he hasn't done a single } \\
\text { physics question in the whole year and he is still going to } \\
\text { get an A theres no way hes going to fail hes taking the pi*s } \\
\text { out of all you }\end{array}$ & im. - r \\
\hline
\end{tabular}

This clearly is the first example for dysfunctional, inappropriate behavior between message board interlocutors. We can even speak of rude behavior (" $r$ ") in all three cases, since the intention to hurt and discredit the other is obvious. Faced with a small string of negatively marked utterance after negatively marked utterance, we could call the entire incident a flame.

The second time $i$ chimes into the conversation, he welcomes another user into the society. Interestingly, and true to his two previously discussed posts ( $\mathrm{p} 1$ and p5), his third contribution also exhibits marked behavior - this time, however, it is positively marked in the form of mock-impoliteness:

\begin{tabular}{|c|c|c|c|}
\hline$W:$ & $\mathrm{p} 21$ & $\begin{array}{l}\text { I'm going to get a B in maths, hist and eng lit } \Theta \text { which } \\
\text { means I wont get into Uni } \Theta \Theta O[\backslash] \text { Can i join too } \\
\text { please } \Theta\end{array}$ & im. $\varnothing$ \\
\hline$i:$ & $\mathrm{p} 22$ & No. Only joking yes. & im. $+\mathrm{m}$ \\
\hline
\end{tabular}

This second and last of the thread starter's involvements in the thread is also exceptional insofar as he only allows one user explicitly to become part of the society - even though most posts contain exactly the same wish. In this regard, thread \#18 pretty much resembles its predecessor, since most users' responses consist in the wish to join the society - again phrased in many different ways. In 
a lot of cases, this wish is accompanied by remarks or worries about the subject they are going to fail or already have failed. The second largest group of responses comprises those users who do not utter the wish to join but express their worries and feelings of hopelessness right away. Then there are also those who look for someone with similar problems and those who try to create a more positive atmosphere by offering help, wishing luck and cheering others up. Since this is a special feature of this thread, here are some examples:

YOUR NOT GOIN TO FAIL

WTF are you doing in here? - get out Lu [lighten up]! 9

9 Best of luck for tomorrow anyway, It's not worth getting worked up the day before $=$ )

[...] I’m sure you'll all do FANTASTIC! If I can pass, anyone can! Yay!

Come on guys, this thread is so pessimistic and melancholic 8 ! If you BELIEVE you will pass, then you will! I know it is cliched, but I'm sure it works! [ $\backslash]$ If you revise and work hard in lessons then why will you all fail? 32 [ 1$]$ POSITIVE THINKING!!

The first example surely strikes as odd, mostly because the utterance is written in capitals, which is usually used for screaming in CMC. The calming influence of this post is thus highly questionable and also not met with a response. The same holds true for the second example, which also borders on inappropriate behavior, as can be told by the following reaction: "as in get out of this thread or get out of TSR 20" (p55). Another maybe naïve but still appropriate attempt to make others feel a little better is p14 ("im. Ø”), "Nobody here is going to fail., eliciting the response "What a stupid thing to say." (p15, "im. - r"), which substantiates users' general tendency to fly off the handle easily in this thread. For this reason, advice sometimes mingles with patronizing and mocking behavior, thus crossing the line to inappropriate behavior:

well i know that iam succesful and iam not allowed on this thread!!!!! but there is nothing that you can do. $[\mathrm{I}]$ imagine that $\mathrm{i}$ do not think that you are serious people.... once you fail just work harder and you will be better $\mathrm{i}$ think...if not then you are dommed $\oplus$ ha ha ha

Other reactions to the thread start include:

- self-pity: [...] Im doomed! (p9)

- self-mocking: Hey I’m gonna fail higher maths, art \& design \& chemistry (its bloody difficult). haven't even sat the exams yet $:-$ there's positive thinking for ya $\because(\mathrm{p} 74)$ 
- calculated optimism: ${ }^{\star}$ thinks posativly ${ }^{*}$ i might just fail my psychology unit 6" (p17)

- comments on the entire thread: This thread just cheered me up $\oplus$ (p87)

_ joking: i need to join this soc too 8 [two breaks] i hate august 6 i really don't want to know my results 8 why aren't we allowed to burn 'em?? (p157)

- explicit emotional display: I feel awful,I’ve just remembered some of my physics phy4 mistakes and it made me feel awful $\mid \Theta$ it made me panic more and I'm very scared ( $\mathrm{p} 120)$

Finally, there are also those users who counteract the instructions of the thread starter and deliberately distance themselves from the apocalyptic mood - again, more often than not to the detriment of the desperate folk and with an insult or an admonition to follow hard on the heels:

Nope, I'm going to pass them all.

well i’m not going to fail anything, just might not get into uni

\begin{tabular}{|l|l|l|l|}
\hline C: & p44 & $\begin{array}{l}\text { [quote: Nobody here is going to fail.] [I] lol! [I] [quote: ooh } \\
\text { ooh can I join?.....Im gonna fail physics especially....had a } \\
\text { practical exam today... [...] I dont think I did too well..coz } \\
\text { I was so p*ssed off....grrrr] [\] lol again!! [I] sorry - good } \\
\text { luck everyone. Sorry if i don't join, but I'm probably not } \\
\text { going to fail - i probably won't do as well as everyone } \\
\text { thinks, but not fail thank god. }\end{array}$ & ex \\
\hline$a:$ & $\mathrm{p} 49$ & $\begin{array}{l}\text { why do you think your better than everyone else? you } \\
\text { didn't get into oxford and you only applied to Aston so } \\
\text { now you have to go to Aston how stupid is that lol. }\end{array}$ & im. - \\
\hline
\end{tabular}

\begin{tabular}{|c|c|c|c|}
\hline$w:$ & p 12 & $\begin{array}{l}\text { Im going to pass my exams, and gets As in them - hahaha- } \\
\text { haha u f } \mathrm{f}^{*} \text { cking crybabies (p12) }\end{array}$ & ex. $-r$ \\
\hline$J:$ & $\mathrm{p} 13$ & $\begin{array}{l}\text { Wow, they just give 'em away these days, don't they. [I] } \\
\mathrm{F}^{*} \text { cker. }[\backslash] \oplus(\mathrm{p} 13)\end{array}$ & im. + m \\
\hline
\end{tabular}

The question of whether we are faced with mock-impoliteness, impoliteness or even rudeness in the second part of the latter exchange can be answered at least approximately with a look at contextual information (see Chapter 6), which provides some hints for likely evaluations of these two utterances. With $w$ being a new member, who is already banned, and $J$ an experienced user with an extremely high reputation, chances are good that at least $J$ did not have a hurtful intention in mind. Both utterances are evaluated accordingly. 
As the next excerpt demonstrates, malevolent members also use mock-politeness in this thread, thus providing us with an example for the last possible type of relational work:

\begin{tabular}{|l|l|l|l|}
\hline q: & p134 & $\begin{array}{l}\text { I'm going to fail my exams. I'll never finish all my music } \\
\text { exam grades by next year } \\
\text { A levels. }\end{array}$ & im. $\varnothing]$ I'm also going to fail my \\
\hline J: & p135 & good for you (马) & im. - m \\
\hline q: & p136 & Yes very good for me 9 & im. $\varnothing$ \\
\hline
\end{tabular}

In terms of facework, there are basically three types of respondents: The lamenting folks (highest proportion), who think they will fail and try to find like-minded people and comfort. Their behavior is appropriate for the purpose of the thread and mirrors their need for association. The insulting folks (low proportion) mock and insult the lamenting folks, partly bragging with their own success. Their behavior is inappropriate, mostly even negatively marked behavior to the disadvantage of the lamenting folks. Finally, there is the comforting folks (low proportion), who encourage and comfort the lamenting folks with the help of appropriate, partly even positively marked behavior in favor of the lamenting folks' need for association. Interestingly, the moderators in this thread behave rather unobtrusively and do not call rowdies to order.

In order to end on a positive note and to illustrate the facework of a member of the comforting folk, one last example shall be presented for this thread:

\begin{tabular}{|c|c|c|c|}
\hline A: & p52 & $\begin{array}{l}\text { Can't sleep because I'm too worried about my maths exam } \\
8\end{array}$ & im. $\varnothing$ \\
\hline$m:$ & p53 & Aww, but Maths is cool. So so cool. & im. $\varnothing$ \\
\hline A: & p54 & It's not cool when you need a B, and are failing. & im. Ø \\
\hline$m:$ & p56 & $\begin{array}{l}\text { It pains me to hear that. } 6[\mathrm{I}] \text { I guess it's a little late to } \\
\text { offer my help...? }\end{array}$ & im. + \\
\hline A: & p57 & $\begin{array}{l}\text { Are you a maths genius? Do you know a brain surgeon? } \\
6 \text { ? }\end{array}$ & im. $\varnothing$ \\
\hline
\end{tabular}




\begin{tabular}{|c|c|c|c|}
\hline$m:$ & p58 & $\begin{array}{l}\text { I do A-level Further Maths, so I could've come in useful. = } \\
{[\backslash] \text { Don't worry. I'm sure I could become a brain surgeon, }} \\
\text { unlock the secrets of time travel and bam! All sorted. [\] } \\
\text { Alternatively, get some sleep. Exam tomorrow? }[\backslash] \text { Worry- } \\
\text { ing about it won't help now. You've learned as much as you } \\
\text { can learn. Just do your best. }\end{array}$ & im. + \\
\hline A: & p60 & $\begin{array}{l}\text { Friday afternoon. I'm going to study as much as I Can til } 9 \\
\text { tomorrow night. } 8\end{array}$ & im. $\varnothing$ \\
\hline$m:$ & p61 & $\begin{array}{l}\text { Hmm. Well I'll be on MSN pretty much throughout the } \\
\text { course of the day tomorrow (because I have no sense, and } \\
\text { lack serious will power!). So, you know where to find me. } \\
=\text { ) }\end{array}$ & ex. + \\
\hline$A:$ & p62 & Thanks $:$ Might need some last minute hints & im. $\varnothing$ \\
\hline
\end{tabular}

Unlike all the other threads dealt with so far, this one was not only characterized by functional communication. Accordingly, the final account (see Table 23), which factors in the interpersonal value of every single post, bears testimony to that.

As the matrix reveals, thread \#18 contains 13 cases of inappropriate behavior, three of which have even been explicitly evaluated as such by interlocutors. What is also interesting is the fact that five out of 13 cases of negatively marked behavior could not only be classified as impolite, but as downright rude. Still, appropriate behavior was predominant even in this thread and could be counted almost twelve times as often as inappropriate behavior. Within this category, unmarked, appropriate behavior clearly outstripped all forms of positively marked behavior with 130 to 24 incidences.

Table 23. Relational work in thread \#18

\begin{tabular}{|c|c|c|c|c|c|}
\hline \multirow{2}{*}{$\begin{array}{l}\text { Type of post } \\
\text { and evaluation }\end{array}$} & \multicolumn{3}{|c|}{ Functional $\rightarrow$ appropriate } & \multicolumn{2}{|c|}{ Dysfunctional $\rightarrow$ inappropriate } \\
\hline & $\begin{array}{l}\text { marked + } \\
\text { "polite" }\end{array}$ & $\begin{array}{c}\text { marked }+\mathrm{m} \\
\text { "mock-impolite" }\end{array}$ & $\begin{array}{c}\text { unmarked } \\
\varnothing\end{array}$ & $\begin{array}{l}\text { marked - } \\
\text { "impolite" } \\
\text { ("rude" = r) }\end{array}$ & $\begin{array}{l}\text { marked - m } \\
\text { "mock-polite" }\end{array}$ \\
\hline A: 54 ex. & 4 & 1 & & $3(2 r)$ & 0 \\
\hline im. & 6 & 1 & 37 & $2(1 \mathrm{r})$ & 1 \\
\hline $\mathrm{B}_{\mathrm{t}}: 113 \mathrm{im}$. & 10 & 3 & 93 & $7(2 \mathrm{r})$ & 0 \\
\hline$\sum: \quad 167(+5 \mathrm{C})$ & 20 & 4 & 130 & $12(5 r)$ & 1 \\
\hline & \multicolumn{3}{|c|}{154} & \multicolumn{2}{|c|}{$13(5 r)$} \\
\hline
\end{tabular}


Incidentally, the analysis of this thread brought us also the first bunch of type $\mathrm{C}$ posts, which shall not remain unmentioned. The author of $\mathrm{p} 87$ describes his change of mood after reading through the thread: "This thread just cheered me up 1 ". Then again others address more or less the entire cast of the thread, such as p131, "Come on guys, this thread is so pessimistic and melancholic $8[\ldots]$ ". Although capturing the mood of the entire thread, these posts still dropped out of the final calculation, as they do not directly refer to any other of their surrounding posts. These examples prove, however, that not every type $\mathrm{C}$ post is automatically unrelated to the content of the ongoing discussion. Of course, there are also "classic" type C posts, such as $\mathrm{p} 38$, in which user $D$ - totally unrelated to previous posts - utters the following request: "I am Boared Can I Talk Tio U", to which no one bothers to react.

When summarizing the entire category, Looking for like-minded others, several new insights in contrast to the first category strike the eye. In responding to the initial post, users

- $\quad$ signal their interest in the group by uttering the wish to join or by joining;

- look for others with similar problems;

- state opinions and express feelings;

- create a positive atmosphere by cheering others up, offering help and wishing luck;

- create a negative atmosphere by patronizing, mocking and even insulting others.

The thread starter's behavior differed considerably within this category: While the initiator of the first thread (\#19) composed 18 out of 75 posts, thus assuming an active role in the conversational flow, the second thread starter appeared very withdrawn with only two out of 172 posts. In general, thread starters

- welcomed their fellow users;

- evaluated their responses and shared information;

- tried to keep the conversation going (only \#18).

Finally, it is worth mentioning that this category does not only exhibit cases of positively marked and unmarked behavior, but also incidences of "the dark side". Still, they do not carry much weight with 228 to only 13 examples of appropriate versus inappropriate behavior respectively. Above that, thread \#18 saw the first specimen of type $\mathrm{C}$ posts, which could not be factored into the analysis due to the lack of anaphoric as well as cataphoric reference. In figures, this can be expressed as shown in Table 24 . 
Table 24. Relational work in the second category (Looking for like-minded others)

\begin{tabular}{|c|c|c|c|c|c|}
\hline \multirow{2}{*}{$\begin{array}{l}\text { Type of post } \\
\text { and evaluation }\end{array}$} & \multicolumn{3}{|c|}{ Functional $\rightarrow$ appropriate } & \multicolumn{2}{|c|}{ Dysfunctional $\rightarrow$ inappropriate } \\
\hline & $\begin{array}{l}\text { marked + } \\
\text { "polite" }\end{array}$ & $\begin{array}{c}\text { marked + m } \\
\text { "mock-impolite" }\end{array}$ & $\begin{array}{c}\text { unmarked } \\
\varnothing\end{array}$ & $\begin{array}{l}\text { marked - } \\
\text { "impolite" } \\
\text { ("rude" = r) }\end{array}$ & $\begin{array}{l}\text { marked - m } \\
\text { "mock-polite" }\end{array}$ \\
\hline \multirow{2}{*}{ A: $98^{\star}$} & 6 & 0 & & $3(2 r)$ & 0 \\
\hline & 7 & 1 & 78 & $2(1 \mathrm{r})$ & 1 \\
\hline $\mathrm{B}_{\mathrm{t}}: 143 \mathrm{im}$ & 13 & 3 & 120 & $7(2 r)$ & 0 \\
\hline \multirow[t]{2}{*}{$\sum: 241(+5 \mathrm{C})$} & 26 & 4 & 198 & $12(5 r)$ & 1 \\
\hline & \multicolumn{3}{|c|}{228} & \multicolumn{2}{|c|}{$13(5 r)$} \\
\hline
\end{tabular}

*+ 1 exception \#19p53: im. Ø, ex. +

\subsubsection{Asking for advice}

In this category, participants are mostly explicitly asked for advice for a problem put forward by the thread starter. In sharing their pearls of wisdom and trying to help the initiator of the thread, TSR users seldom engage in lively discussions, thus hardly producing dialogic structures. This is also mirrored in the statistics for a category that comprises the second largest number of items in the entire corpus: Of the sum total of 16 threads, 12 (75\%) exhibit a low IQ, with the remaining 4 threads (25\%) featuring a medium IQ. Surprisingly, there are no threads with a high interaction quotient. Similar results can be spotted when looking at the number of posts per day as an indicator of the attraction of the content of the opening post: As a matter of fact, 11 out of 16 threads stayed beneath the average of 12.5 $\mathrm{p} / \mathrm{d}$, thus receiving responses only extremely hesitantly (see, for example, thread \#34, Pros and cons of boarding school, with only $0.1 \mathrm{p} / \mathrm{d}$ or thread \#40, Veganism, with $0.2 \mathrm{p} / \mathrm{d}$ ). The other five threads attracted more contributions per day, thus sporting a high amount of $\mathrm{p} / \mathrm{d}$ such as thread \#11, What does this girl mean by this? (convo included), with a whooping $40 \mathrm{p} / \mathrm{d}$. To illustrate this category, thread \#14, How to stop strange people speaking to me (IQ: $24 \%$, p/d: 27) will be picked:

\section{How to stop strange people speaking to me}

OK, so I'm doing a prjoect over the summer (a Nuffield Bursary) at a place that's an hour and a half away by train. Been there for two and a half weeks, got a week and a half left. [ $\backslash]$ Last week as I was walking to the station, this 12 year old kid asked me for my number and this 40 year old wolf-whistled at me, but that was fine because I could just walk away. [another incident] [ $\mathrm{I}$ ] Today however, another guy spoke to me. I'd say he was in his mid-twenties, single (he told me) and he sat a lot closer than the other guy - opposite me 
in a 4 - but the train was practically empty and I saw him walk down the carriage past loads of empty seats. He said he'd seen me sitting alone on the train before and he started speaking to me. He didn't ask creepy questions like "Are you a virgin?" or whatever (I've had that before) but he talked about seeing me later/tomorrow and invited me to his barbershop tonight when I finish my project for the day. [ $\backslash$ ] Now of course I'm not going to go, but I'm concerned that I may see him tomorrow or over the next week and he'll keep being friendly. I'd much rather sit alone on the train! Also he might ask why I didn't turn up. I don't want any trouble, particularly because by the time he gets on (half an hour from my destination, which is the last stop) it's almost empty. [weighing options to avoid the guy] [ $\mathrm{I}$ I don't wear revealing clothes or makeup, I'm not pretty and when they talk to me I always act even more shy than I am naturally, so I don't know why people talk to me. It's not like I ask for it! I always give false details so they can't track me down, but that doesn't stop people recognising me on the journey. [ $I$ ] So what can I do to stop this guy and people in general speaking to me? I don't mind friendly chats like with the bloke last night but I don't want to develop a 'friendship' with this other guy. Of course I could say tomorrow's my last day or something, but then if he sees me next week..? [ $\backslash]$ Sorry for the long post.

(Anonymous User \#1, thread \#14, post 1)

The opening post of thread \#14 stands out against most other initial posts because of its length (which is why it needed to be shortened here). Even the thread starter, Anonymous user \#1 (A), acknowledges this violation of the TSR code of conduct and apologizes with the metapragmatic utterance "Sorry for the long post". The rather obscure thread starter, who does not disclose anything about him- or herself, has already been suspected to be a troll (see Section 7.3.2), since all four threads set up by him contain diverging face claims thus pointing to different, maybe deliberately faked personas. In this thread, $A$ plays the part of a girl who narrates her problem in detail before asking her co-participants for advice. She does so in a well-structured way, abiding by orthography and punctuation. Not only can we find hardly any typos but also no signs of netlingo, at least the latter being unusual for message board entries.

A sum total of 53 responses were elicited by this initial post - among them only two follow-up contributions by the thread starter, who only intervenes to justify her position and to thank everyone present for their helpful suggestions to get rid of the man on the train. The latter is the most prominent of all possible reactions:

Easy, whenever someone is about to sit next to you on a bus/train, simply pat the seat whilst, raising your eyebrows and grinning leeringly. Works for me 
every time. A pox to the fat person who dares to try and sit next to me on the bus

Invest in an mp3 player. Works a charm.

Stare out the train window therefore not inducing any conversation.

Headphones.

Others, however, react less understanding and helpful, laying the blame on the thread starter herself. Some of them are presented with a corresponding answer immediately:

Wow, women sure can be paranoid...

put down the giant sign, saying, 'talk to me'.

funny how guys wouldn't mind at all if a girl spoke to them, but if its the other way around, girls bitch, stfu [= shut the fuck up]

\begin{tabular}{|l|l|l|l|}
\hline 9: & p5 & $\begin{array}{l}\text { you are the reason why guys dont talk to girls in public } \\
\text { places... }\end{array}$ & ex. - \\
\hline A: & p33 & $\begin{array}{l}\text { Gee, wow thanks. If a guy roughly my age approached me, } \\
\text { say, aged } 15 \text { to } 19, \text { I'd be more chatty even though I have a } \\
\text { boyfriend, simply because it's nice to make friends. Quite } \\
\text { frankly though, I don't want guys } 10 \text { years older than me } \\
\text { trying to be my mate. [...] }\end{array}$ & im. \\
\hline
\end{tabular}

While sharing strategies, some users discover commonalities with their interlocutors and contribute their own experiences:

ब्व $[\backslash]$ I'm not the only one!

it can be flattering until it gets to that uncomfortable point $[\backslash]$ i always feel unhapy when a person interest in me doesn't equate to the how well they know me $[\backslash]$ i struggle to say no too but because you can't be too careful this day and age $i$ will always say $i$ have a boyfriend who fancy very much because of how strong he is $[\backslash]$ it can also be insulting that people think you can be won over that easily i suppose

OP, tell them to ${ }^{* * *}$ off. it's only a stranger, so who cares? (1) [ $\left.\backslash\right]$ I remember one time, some 16 year olds were on a bus with me and asked me "yo blud, you got a cigarette?" i said "no, i don't give money to low-class chav people". they wanted to hit me, but they got off the next stop, and shouted and swore at me as they left. [ $\backslash$ ] I couldn't care less. I didn't know them, he who gets angry loses. 
Then again others comment on the views and/or face claims expressed by their co-participants, or joke about the problem described:

Balaclava.....nobody sits next to someone wearing a balaclava. Oh the fun of it... [...]

cover yourself in manure, im sure nobody would want to sit next to you if you stink.

\begin{tabular}{|c|c|c|c|}
\hline$n:$ & p9 & $\begin{array}{l}\text { You can tell them to foff. Wouldn't work on me, but on } \\
\text { other less confident guys. MP3 Player works well too. But } \\
\text { that ain't ever stopped me either, so really just be polite and } \\
\text { if they creep you out move. }\end{array}$ & $\operatorname{im} . \varnothing$ \\
\hline$S:$ & p14 & $\begin{array}{l}\text { You do realise how bad you make yourself sound right? } \\
{[\ldots]}\end{array}$ & $\operatorname{im.~} \varnothing$ \\
\hline$n:$ & p18 & $\begin{array}{l}\text { Last time I hit on a girl on a train she orginally told me to } \\
\text { f off. } 20 \text { minutes later she was giving me her number and } \\
\text { asking for mine. I'm good at what I do. }\end{array}$ & im. $\varnothing$ \\
\hline$G:$ & $\begin{array}{l}\mathrm{p} 21 \\
(\rightarrow 18)\end{array}$ & $\begin{array}{l}\text { It was probably a fake number to make you }{ }^{* * * *} \text { off. }[\backslash] \text { To } \\
\text { be honest, I feel terrible for the girls who have to put up } \\
\text { with desperate guys constantly trying to hit on them when } \\
\text { they're just trying to get from A to B. There's something } \\
\text { very creepy about having to trap a girl in a moving train } \\
\text { before they will talk to you. [ } \backslash] \text { Even more repulsive is the } \\
\text { fact that most of these guys are completely oblivious to } \\
\text { how uncomfortable they make the poor girls feel. Espe- } \\
\text { cially the idiots who are like "naw man i am usin mar skillz } \\
\text { to get da girlz lol dey like it u see." }\end{array}$ & im. $\varnothing$ \\
\hline$f:$ & $\begin{array}{l}\text { p24 } \\
(\rightarrow 18)\end{array}$ & I really want to see how you do that $\&[\ldots]$ & im. $\varnothing$ \\
\hline$n:$ & $\begin{array}{l}\text { p30 } \\
(\rightarrow 24)\end{array}$ & $\begin{array}{l}\text { I think the specific line I used was, wow, that's very rude. } \\
\text { You this impolite to all strangers. Then i got a sorry, what } \\
\text { did you want. And then we just got talking. Then I had to } \\
\text { get off and she asked for my number and gave me hers. } \\
\text { And it wasn't phony cause she was the one texting me. [ }[] \\
\text { I'm not some creep, I don't do that kind of thing often, but } \\
\text { if I do stick out my neck, a girl trying to insult me before } \\
\text { I've spoken a single word kinda means nothing. Of course } \\
\text { after a minute if she still hatin, it's me that shes hating, not } \\
\text { the being approached part. }\end{array}$ & im. $\varnothing$ \\
\hline
\end{tabular}




\begin{tabular}{|l|l|l|l|}
\hline$p:$ & $\begin{array}{l}\mathrm{p} 28 \\
(\rightarrow 18)\end{array}$ & $\begin{array}{l}\text { OMG i hate guys like you. Are you stupid enough to not } \\
\text { the get the msg if she swore at you? I bet it was a fake num- } \\
\text { ber cos I do it all the time } \Psi\end{array}$ & im. $-\mathrm{r}$ \\
\hline
\end{tabular}

In offering advice to the thread starter, $n$ provoked a multilayered discussion about his character in $\mathrm{p} 9$, basically dealing with the negotiation of his face claims between himself and six other participants. Here is the second string of dialogue elicited by $\mathrm{p} 9$ :

\begin{tabular}{|c|c|c|c|}
\hline$n:$ & p9 & $\begin{array}{l}\text { You can tell them to foff. Wouldn't work on me, but on } \\
\text { other less confident guys. MP3 Player works well too. But } \\
\text { that ain't ever stopped me either, so really just be polite and } \\
\text { if they creep you out move. }\end{array}$ & im. $\varnothing$ \\
\hline$F:$ & p22 & $\begin{array}{l}\text { If a girl told you to } \mathrm{f} \text { - off you wouldn't take a hint and leave } \\
\text { her alone? } 68\end{array}$ & $\operatorname{im} . \varnothing$ \\
\hline$n:$ & p29 & $\begin{array}{l}\text { If she told me after a minute or so. But if the opening line } \\
\text { is F-off then yeah, I'll try my luck for a bit. That's just how I } \\
\text { am. If i don't get anywhere after like } 30 \text { seconds I'll apolo- } \\
\text { gize and cut my losses. }\end{array}$ & im. $\varnothing$ \\
\hline T: & p31 & $\begin{array}{l}\text { People who boast about such things on the internet are } \\
\text { generally compensating for their failures in life. }\end{array}$ & im. $-r$ \\
\hline$n:$ & p32 & $\begin{array}{l}\text { Lol, I'm not boasting. I was just replying to some state- } \\
\text { ments. But who cares. it's the internet! } \bigoplus[] \text { And yes, like } \\
\text { everyone else I have my own failures in life. I know my } \\
\text { short comings, no need to tell me. }\end{array}$ & im. $\varnothing$ \\
\hline
\end{tabular}

In summarizing the exemplary thread for this category, we obtain the following figures, which correspond with previously gained results in terms of the distribution of appropriate and inappropriate behavior. Interestingly, in most cases of negatively marked behavior, the intention to hurt the other's face was quite obvious, which is why these posts earned the label rude (see Table 25).

Naturally, the analysis of the other 15 threads belonging to this category has brought to light more examples for the facework strategies as discussed for thread \#14. Above that, more strategies, positive as well as negative ones, could be detected in the behavior of thread starters and their interlocutors likewise. Classified into groups, one might summarize them in the following, far from exhaustive list: 
Table 25. Relational work in thread \#14

\begin{tabular}{|c|c|c|c|c|c|c|}
\hline \multirow{2}{*}{\multicolumn{2}{|c|}{$\begin{array}{l}\text { Type of post } \\
\text { and evaluation }\end{array}$}} & \multicolumn{3}{|c|}{ Functional $\rightarrow$ appropriate } & \multicolumn{2}{|c|}{ Dysfunctional $\rightarrow$ inappropriate } \\
\hline & & marked + & marked $+\mathrm{m}$ & unmarked & marked - & marked - m \\
\hline \multirow[t]{2}{*}{ A: 15} & ex. & 0 & 0 & & 1 & 0 \\
\hline & $\mathrm{im}$. & 1 & 0 & 12 & $1(1 \mathrm{r})$ & 0 \\
\hline $\mathrm{B}_{\mathrm{t}}: \quad 39$ & im. & 2 & 0 & 33 & $4(3 r)$ & 0 \\
\hline \multirow[t]{2}{*}{$\sum: \quad 54$} & & 3 & 0 & 45 & $6(4 r)$ & 0 \\
\hline & & \multicolumn{3}{|c|}{48} & \multicolumn{2}{|c|}{$6(4 r)$} \\
\hline
\end{tabular}

A. positive facework, i.e. in favor of the need for association or dissociation:

- giving helpful suggestions, advice and/or warnings to solve the problem and/or to answer the initial question;

- contributing own experiences;

- thanking others for suggestions;

- discussing impressions (face claims) of others;

- discovering commonalities, sharing worries and feelings, teaming up;

- reassuring and encouraging others;

- leaping at someone's defense;

- commenting on others' views by either supporting and agreeing with them or by correcting or contradicting them;

- enjoying others' jokes;

- discussing (more or less seriously) the code of conduct in TSR;

- dissolving misunderstandings by asking for and giving clarifications;

- praising formal aspects such as others' signatures.

B. negative facework, i.e. to the detriment of the need for association or dissociation:

- laying the blame for the problem on the one with the problem (mostly the thread starter);

- not helping but joking instead;

- mocking the other (also with the help of smileys, inappropriate links and pictures);

- insulting the other bluntly;

- commenting negatively on the form of the post;

- suspecting the other to be a troll - verbally and pictorially;

- criticizing the content of the other's post. 
Table 26. Relational work in thread \#11

\begin{tabular}{l|c|c|c|c|c}
\hline \multirow{2}{*}{$\begin{array}{c}\text { Type of post } \\
\text { and evaluation }\end{array}$} & \multicolumn{3}{|c|}{ Functional $\rightarrow$ appropriate } & \multicolumn{2}{c}{ Dysfunctional $\rightarrow$ inappropriate } \\
\cline { 2 - 6 } & $\begin{array}{c}\text { marked }+ \\
\text { "polite" }\end{array}$ & $\begin{array}{c}\text { marked }+\mathrm{m} \\
\text { "mock-impolite" }\end{array}$ & $\begin{array}{c}\text { unmarked } \\
\varnothing\end{array}$ & $\begin{array}{c}\text { marked }- \\
\text { "impolite" } \\
\text { ("rude" }=\mathrm{r})\end{array}$ & $\begin{array}{c}\text { marked - m } \\
\text { "mock-polite" }\end{array}$ \\
\hline $\mathrm{A}: 28^{*} \mathrm{ex}$. & 0 & 0 & $6(4 \mathrm{r})$ & 1 \\
\hline $\mathrm{im}$. & 0 & 0 & 14 & $5(5 \mathrm{r})$ & 2 \\
\hline $\mathrm{B}_{\mathrm{t}}: 88 \mathrm{im}$. & 3 & 0 & 34 & $49(42 \mathrm{r})$ & 2 \\
\hline$\sum: 116(+2 \mathrm{C})$ & 3 & 0 & 48 & $60(51 \mathrm{r})$ & 5 \\
\hline & & 51 & & \multicolumn{2}{c}{$65(51 \mathrm{r})$} \\
\hline
\end{tabular}

*+2 exceptions p1, 7: im. Ø, im. $-\mathrm{r}$

A lot of examples for the negative side of facework stem from thread \#11, a thread which poses an exception insofar as its originator was suspected to be a troll at all times. For this reason, almost every reaction was downright hostile with only a small proportion of posts trying to deal with the actual question seriously. Consequently, the figures depicting the relational work in this thread (see Table 26) speak for themselves.

Never before has a thread contained so many instances of inappropriate, dysfunctional posts, with impoliteness and mock-politeness, and most of all rudeness leading the way. Indeed, this is the only thread in which there are more instances of inappropriate behavior than appropriate behavior - a reliable indicator for the fact that the bulk of participants considered the thread starter and the content of his opening post to be a fraud.

Beside this notable exception, there were other exchanges worth mentioning for one reason or another: In answering the initial question of the thread starter, a user in thread \#43 stated his opinion without criticizing or even addressing anyone else: "I've no intention on changing whatsoever. Why should I? It's my life, not anyone else's." (p8). Still, someone must have taken offence in this post, as the user received a negative reputation (i.e. he was "negged"), leaving him wondering "And why was I negged for this? $\stackrel{m}{-2}$ " (p17). This goes to show that it is not always easy - not even for users of TSR - to separate between a disagreement on a content-related plane and one on an interpersonal plane. In theory, only the latter should be relevant for the reputation system, whose purpose is not to reprimand users for (diverging) opinions but only for misconduct.

In thread \#16, we encounter users who gang up against the thread starter to mock him. What is interesting about their behavior, though, is the fact that with 
their "laughter" (basically "lol" and smileys), they mutually evaluate their conduct as appropriate. They do so with a view to the opening post, which contains a question that can indeed hardly be taken seriously (heading: "What does it mean if a girl smiles at you?"; first post: "What is she thinking?"). Accordingly, the label appropriate has a different value in this particular thread. Hence, it can also be attached to a post, which would have been estimated differently in other contexts. As noted before, this example illustrates that appropriateness really lies in the eye of the beholder.

The same thread holds another cluster of posts worth discussing, since they can be considered one of the very rare cases of a cut and dried evaluation. With reference to two previous posts, which answer the thread starter's question more than frivolously, this particular TSR member types underneath both quotes "Mean!" (\#16p51), thereby clearly evaluating the interpersonal effect of his two predecessors' entries. Other examples for beautifully outspoken evaluations of a preceding post can be found in thread \#15, in which the entry "and if, at 18 , you've not ever even touched alcohol, your parents much be so so obsessive about controlling what you eat - never had good chocolate mousse or anything?” (p53) is followed up with these two comments: "Totally uncalled for og" (p54) and "Couldn't really let this one go. $[. .$.$] " (p63). It is cases like these which are$ ideal for a post-hoc evaluation, since users' views and interpretations are virtually committed to writing.

An interesting contradiction of form and content can be detected in thread \#49. Proving that there is indeed no constant connection between these two, a user literally screams at his interlocutor with a large font size (36pt) and bold capitals - which usually counts as rude behavior - just to encourage him. The reaction of the one shouted at confirms the positive effect of this rather unusual facework strategy, though:

E: DO WHAT YOU WANT [original: 36pt] [ $\mathrm{I}$ ]'m tired of answering this question. Your an adult [original: 36pt], you don't need your parents to run your life anymore. It's entirely up to you what you do with your time.

M: Fantastic! That made me smile $(;)$

Last but not least, a very explicit statement about how to treat other users online, posted in thread \#42, shall not be left out either. At the end of a heated argument between two participants, which could also qualify as a flame, one of the users leaves the following remark:

[...] I'm not insecure, I don't even know who you are, I just don't really see any reason why I need to be espescially polite or civil towards you. My social skills are fine, but this is the internet and I'm not really looking to make friends with 
you or anyone like you. There's nothing to gain from kissing your arse and there's nothing I lose from being impolite to you.

(\#42p58)

The simple fact that most other users do behave when interacting with their fellow members, even become friends, signals that this opinion is not shared by the majority. The figures for the entire third category (see Table 27) support this claim. Even thread \#11 (the troll-thread) could not change the general outcome of all 16 threads. As noted before, cases of appropriate behavior outstrip cases of inappropriate behavior at the rate of 6:1 approximately. While examples of both mock-politeness and mock-impoliteness are rare, it is again unmarked behavior which surpasses all other types by far. Even the relatively high amount of 49 incidences of politeness (see Table 27) cannot change that.

A look at the seven exceptions reveals the obvious: Evaluations tend to vary between marked and unmarked behavior in the eyes of the interlocutors. Although the biggest possible contrast could not be detected in this batch of threads - i.e. the contrast between positively and negatively marked behavior - some posts at least managed to elicit such diverse evaluations as unmarked vs. rude.

On a marginal note, this category saw three of those thread starters in action that excelled either by their positive or negative reputation (see Section 7.3.2). Two of them, Geritak (\#15) and Sahds (\#42), both of whom were labeled "do-gooders" previously, did not leave an impression at all, as they hardly intervened in the threads created by them. The third of them, the "trouble maker" $9 \mathrm{MmBulletz}$

Table 27. Relational work in the third category (Asking for advice)

\begin{tabular}{|c|c|c|c|c|c|}
\hline \multirow{2}{*}{$\begin{array}{l}\text { Type of post } \\
\text { and evaluation }\end{array}$} & \multicolumn{3}{|c|}{ Functional $\rightarrow$ appropriate } & \multicolumn{2}{|c|}{ Dysfunctional $\rightarrow$ inappropriate } \\
\hline & $\begin{array}{l}\text { marked + } \\
\text { "polite" }\end{array}$ & $\begin{array}{c}\text { marked + m } \\
\text { "mock-impolite" }\end{array}$ & $\begin{array}{c}\text { unmarked } \\
\varnothing\end{array}$ & $\begin{array}{l}\text { marked - } \\
\text { "impolite" } \\
\text { ("rude" = r) }\end{array}$ & $\begin{array}{l}\text { marked - m } \\
\text { "mock-polite" }\end{array}$ \\
\hline \multirow[t]{2}{*}{ A: $210^{*}$} & 10 & 0 & & 17 (10r) & 1 \\
\hline & 6 & 0 & 166 & $8(7 r)$ & 2 \\
\hline $\mathrm{B}_{\mathrm{t}}: 536 \mathrm{im}$ & 33 & 3 & 426 & $70(48 \mathrm{r})$ & 4 \\
\hline \multirow[t]{2}{*}{$\sum: 746(+12 \mathrm{C})$} & 49 & 3 & 592 & 95 (65r) & 7 \\
\hline & \multicolumn{3}{|c|}{644} & \multicolumn{2}{|c|}{$102(65 r)$} \\
\hline \multicolumn{6}{|c|}{ 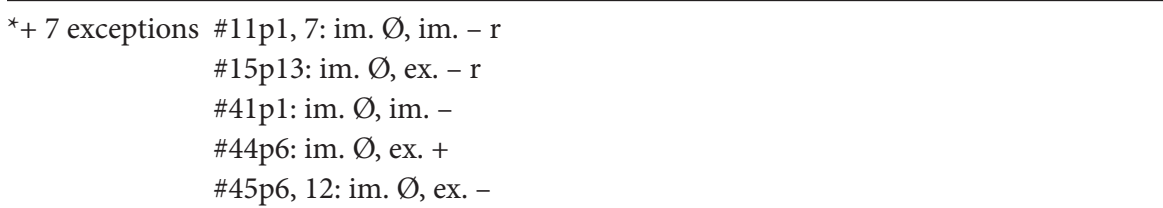 } \\
\hline
\end{tabular}


(\#34), gave us at least something to work with in the form of four further contributions. Although he did not really stir up any trouble in them, a rather demanding and impatient streak was still salient: Seven minutes after his initial entry, he posted "someone?" (p2), approximately half an hour after his first appearance in this thread, he addressed his interlocutors once again rather bluntly by uttering "anyone else?" (p7), thus pushing noticeably unmitigated for answers.

\subsubsection{Asking for opinions}

In this category, high IQs could be expected, as interlocutors are (mostly explicitly) asked by thread starters to exchange their opinions and to discuss a topic. In reality, though, ten out of 23 threads feature a low (43.5\%), eight a medium (34.8\%) and five a high IQ (21.7\%). Despite these figures, the interactive character of this category does shine through, as five of the seven threads in the entire corpus that rank "high" in terms of their IQ can be found in this category. A look at the number of posts per day yields diverging results, since some threads exhibit very high results, such as thread $\# 13$ with $65 \mathrm{p} / \mathrm{d}$, then again others extremely low ones (see, for example, thread \#33 with only $0.4 \mathrm{p} / \mathrm{d}$ ). To illustrate this category, a thread in which participants engage in a heated discussion shall be chosen. Thread \#13 (Do intellectuals put you off?) appears to be the ideal candidate, since it does not only exhibit an elevated IQ of $46 \%$ and a high amount of 65 posts per day. Above that, the thread starter has been found out to be one of the "trouble makers" in Section 7.3.2, which makes this thread even more appealing from an analytical point of view.

\section{Do intellectuals put you off?}

Here's a Q. [ I] Would you be put off by someone if they were a bit of an intellect? [ $\backslash]$ Now I have to make the distinction between someone who is intelligent vs an intellect $[\backslash]$ The latter being someone who chooses to engage themselves in certain pursuits of the mind, and could be classified as a deep thinker, someone who say engages in heated debates and likes to be expressive about their viewpoint and theories and whatnot, being into socio-cultural issues, etc... etc ... [ $\backslash]$ Discuss.

(Adonis, thread \#13, post 1)

Adonis (A) is an extremely savvy TSR user with more than 6000 posts at the moment of posting this contribution. Despite a warnings level of two, he sports a high amount of positive reputation (12 gems). To a certain extent, this documents his behavior in previous exchanges and the varying results yielded in terms of relational work therein. Showing awareness for his effect on others, he even puts 
this face claim into words by crowning himself "Winner of: Most controversial member on TSR" in his profile (see Section 7.3.2). In his opening post, $A$ makes his expectations for the progress of this thread perfectly clear: The question he would like to see answered is not only repeated (heading and entry) but also labeled as such by the metapragmatic statement "Here's a Q". The main part of this entry attempts to define the most central concept of his object of discussion, intellectuals. Afterwards, his fellow-participants are forthrightly asked to discuss by means of a bare imperative.

In complying with this request, 34 experienced participants speak their minds, thus creating 64 subsequent posts, among them five follow-up contributions by the thread starter himself. As usual, only a small proportion of users try to answer the question seriously, exploiting the advantages of netlingo and the means of communication here and there as the following examples attest:

no, i find them very interesting to talk to..so im not put off. $:-;[\backslash]$ but as long as they wernt like that all the time..it's good for convo every now and then.

Depends how keen they are for everyone to know they're intelligent.

Real intellectuals (read: not people who are simply intelligent and go to a top uni) $=$

Agreement with certain lines of argumentations is signaled in a similar manner:

Ha ha I see your point. I meant I have [...]

I know what you mean... hate it when people are patronising on the basis of what seems like your appearance. HATE it.

LOOOOOOL.

What is more, a big part of this thread includes reactions which show that the initial question is not taken seriously and thus answered ironically or jokingly, sometimes even offensively, as the last two excerpts demonstrate:

Yep, definitely. I hate it when people think. Why can't we just walk around naked and do each other at whim.

People's biggest muscle shouldn't be their brain. Time and time again, I've told that to people, sigh. [I] Ok seriously. [I] I don't like it when people [...]

R: I like my girls like I like my horses. Stubborn at first, but easily broken.

O: That'd be all of them then. 
intellectuals, I will hump the first female intellectual i meet- I haven't met any yet.... but i love listening to intellectual people.

In some cases, the thread starter is even mocked for asking the question (in this form) in the first place, leading at times to full-fledged discussions about his or others' face claims:

Great idea asking in a student forum.

I don't think this is something you have to worry about.

\begin{tabular}{|c|c|c|c|}
\hline$C:$ & p7 & $\begin{array}{l}\text { Looks like you've used 'intellect' in place of 'intellectual'. An } \\
\text { intellect is something a person possesses, not what a person } \\
\text { is. [\] Anyhow, the way I look at things, your description } \\
\text { of an intellectual here sounds like a person I can have a } \\
\text { meaningful relationship with (be it platonic or otherwise) } \\
\text { and anyone else I would dismiss as a drone. }\end{array}$ & im. $\varnothing$ \\
\hline A: & p14 & $\begin{array}{l}\text { you know... I should've been more specific and proof-read } \\
\text { things }[\backslash] \text { anywaaaaay }[\backslash] \text { basically... what I meant was }[\backslash] \\
\text { like what someone else pointed out earlier }[\backslash] \text { people who } \\
\text { are bit toooooooo keen on trying to prove their 'intelli- } \\
\text { gence' or overly-analytic types }\end{array}$ & im. $\varnothing$ \\
\hline$W:$ & p63 & Are you a bit dumb? & im. - \\
\hline A: & p64 & nope & im. $\varnothing$ \\
\hline$C:$ & $\begin{array}{l}\mathrm{p} 20 \\
(\rightarrow 14)\end{array}$ & $\begin{array}{l}\text { You must realise that loading a question with two qualifiers } \\
\text { suggesting excess renders it completely pointless. If some- } \\
\text { one is "too" keen and "overly" analytical, that by definition } \\
\text { means that these qualities in them are developed more } \\
\text { than would be desirable in the context of the question, so } \\
\text { the answer that I would be put off is necessitated. [ } \backslash] \text { You've } \\
\text { also changed the question. At first you were talking about } \\
\text { intellectuals, and now you're talking about people who are } \\
\text { attempting to appear intellectual, and there is a big differ- } \\
\text { ence between these types. }[\backslash] \text { Before you accuse me of being } \\
\text { picky, notice that you've started a thread about intellectuals } \\
\text { so you've brought it on yourself. }\end{array}$ & ex. - \\
\hline A: & p32 & $\begin{array}{l}\text { Tis true Mr. Pedantic }[\backslash] \text { I would answer the rest, but you } \\
\text { know me.. laziness }\end{array}$ & im. $-\mathrm{m}$ \\
\hline
\end{tabular}


What is intriguing about the exchange between these three users is not only the fact that they negotiate their face claims but also how these face attributions are taken. $C$ positions himself as a rather pedantic discussant who needs to define the objects of discussion meticulously before actually joining said discussion - a behavior which is outspokenly mocked by $A$ in $\mathrm{p} 32$, thus finishing off the dialogue between these two users. $A$ on the other hand does not live up to his reputation as a "trouble maker" at all, when he does not answer but endorse allegations against his face. Indeed, he agrees when others would have protested (p14) and simply negates when others would have picked a fight (p64). Some posts later, $A$ even admits voluntarily, "[...] on a side note: I swear I have issues communicating [ I ] I know what I wanna say, but it never seems to come out right lol” (p18), thus even reinforcing previous face constitutions.

In fact, neither $A$ nor $C$ are off the hook yet when it comes to negotiating their face claims - the only difference being that $A$ passes the torch to someone else, $I$, as $A$ reports back only once in p31:

\begin{tabular}{|l|l|l|l|}
\hline I: & p21 & $\begin{array}{l}\text { People's biggest muscle shouldn't be their brain. Time and } \\
\text { time again, I've told that to people, sigh. [\] Ok seriously. } \\
{[\backslash] \text { I don't like it when people go overboard with intellectual }} \\
\text { debates. [...] }\end{array}$ & im. $\varnothing$ \\
\hline$C:$ & p25 & $\begin{array}{l}\text { I think you're confusing the majority of people with a genu- } \\
\text { ine interest in 'intellectual pursuits' (as much as I have that } \\
\text { expression - it typically means 'things you have to think } \\
\text { about a bit to take an interest in') with a specific - though } \\
\text { admittedly voal - subset comprising those who [...] }\end{array}$ & im \\
\hline I: & p28 & $\begin{array}{l}\text { Er that's kind of what I thought OP meant. I a bit confused } \\
\text { to what OP means then. }\end{array}$ & im. $\varnothing$ \\
\hline
\end{tabular}

\begin{tabular}{|l|l|l|l|}
\hline C: & p30 & $\begin{array}{l}\text { Well, given that it's a thread by Adonis, it usually means } \\
\text { he's thought of something but not bothered to actually } \\
\text { decide how he's going to put it before posting, write a mess } \\
\text { which people interpret in various ways and then tell people } \\
\text { he meant something else (in an equally ambiguous way) } \\
\text { whenever they answer it in a way that he didn't want them } \\
\text { to. }\end{array}$ & $\begin{array}{l}\text { im. }-\mathrm{r} \\
\text { also: } \\
\text { im. } \varnothing\end{array}$ \\
\hline$I:$ & p32 & $\begin{array}{l}\text { I'm a fairly new member so I don't know much about } \\
\text { Adonis accept he likes weight training. [ }[] \text { oh well. }\end{array}$ & im. $\varnothing$ \\
\hline
\end{tabular}




\begin{tabular}{|l|l|l|l|}
\hline C: & p35 & $\begin{array}{l}\text { He also enjoys looking at oiled up men far more than } \\
\text { any man claiming to be straight should. Probably more } \\
\text { than any man claiming to be gay should. [ } \backslash] \text { Don't worry, } \\
\text { there are actually plenty of us in the fitness forum (myself } \\
\text { included) who enjoy weight training but don't go for the } \\
\text { oily men. }\end{array}$ & im. - r \\
\hline A: & $\begin{array}{l}\text { p31 } \\
(\rightarrow 30)\end{array}$ & $\begin{array}{l}\text { it's funny because it's true lol [ } \backslash] \text { ah [C] making me lol in } \\
\text { real life at 2am [yes my clock is 7 mins fast in adonistan] }\end{array}$ & im. $\varnothing$ \\
\hline p33 & $\begin{array}{l}\text { so basically you're a well liked troll with lots of positive rep? } \\
{[\backslash] \text { I'm rather fond of trolls as they make TSR interesting, }} \\
\text { and seem to rebel in a forum that is governed by so many } \\
\text { strange rules that you have to abide by (I got alerted for } \\
\text { claiming Mr.Blobby was a celebrity hence my thread should } \\
\text { remain in Celeb gossip rather than chat). [I] I don't see why } \\
\text { some people get so worked up by trolls, their threads don't } \\
\text { really have any significance upon their own life do they } \\
\text { (they have the power to ignore them or resist the tempta- } \\
\text { tion to launch in a scathing attack on them). }\end{array}$ & im \\
\hline
\end{tabular}

In this example, users' rather unexpected reactions result in two different evaluations of $\mathrm{p} 30$. Practicing his pedantry yet again, $C$ once more tries to incite $A$, while discussing with $I$. As seen before, $A$ keeps his cool and agrees with $C$ 's evaluation of himself instead of disagreeing and being insulted. Accordingly, p30 can only be labeled "im. Ø" - at least from A's and, for that matter, also from I's perspective. Other participant observers, however, would have estimated this entry as "im. - r" in all likelihood. For that reason, this particular entry deserves two labels and needs to be treated as an exception.

Although C's behavior has escaped direct commentary so far, the next participant, $m$, is not as indulgent as her predecessors. In her conversation with $T$, in which she creates common ground by sharing T's views, she also starts on $C$ s previous entries, renegotiating once more his face claims:

\begin{tabular}{|l|l|l|l|}
\hline$m:$ & p22 & $\begin{array}{l}\text { I know what you mean... hate it when people are patronis- } \\
\text { ing on the basis of what seems like your appearance. HATE } \\
\text { it. }\end{array}$ & ex. + \\
\hline T: & p26 & $\begin{array}{l}\text { Thank you, someone who understands }: \text {. Snap on GCSE's } \\
\text { btw. }:\end{array}$ & ex. + \\
\hline
\end{tabular}




\begin{tabular}{|c|c|c|c|}
\hline$m:$ & p38 & $\begin{array}{l}{ }^{*} \text { High five }{ }^{*} \text { and lets hope the the next set of results follow } \\
\text { suit! } 80[\backslash] \text { The combination of intelligence, sportiness, } \\
\text { a tendncy to be intellectual, good looks and courtesy is } \\
\text { too rare for my liking (-) }[\backslash] \text { And }[C] \text { is doing an excel- } \\
\text { lent job of demonstrating the kind of aggressive, annoying } \\
\text { and pointless pedantry that would put me right off. Thank } \\
\text { you. }[\backslash] \text { (Yes I know this was not the question but I feel its } \\
\text { related. }[A] \text {, everyone else got what you meant }(9)\end{array}$ & $\begin{array}{l}\text { im. }+ \\
\text { (to } T \text { ) } \\
\text { ex. - } r \\
\text { (to } C \text { ) } \\
\text { im. }+ \\
\text { (to } A \text { ) }\end{array}$ \\
\hline$C:$ & $\mathrm{p} 40$ & $\begin{array}{l}\text { Wicked, thanks. One more I can add to the ever-growing } \\
\text { list of women who won't sleep with me because I'm obnox- } \\
\text { ious. }[\mathrm{I}][\ldots]\end{array}$ & $\operatorname{im} . \varnothing$ \\
\hline$m:$ & $\mathrm{p} 44$ & $\begin{array}{l}\text { I understand your need to be so pedantic, your posts } \\
\text { resemble the way I think. But I've learnt to restrain it } \\
\text { because it tends to annoy people and in a lot of cases it's } \\
\text { just pointless really. [ } \backslash] \text { I think this thread has been fairly } \\
\text { coherent for a TSR thread, with most people having un- } \\
\text { derstood the question or having at least made a comment } \\
\text { related in some way. [ } \backslash] \text { I wouldn't worry, no-one properly } \\
\text { understands the world. But it sounds like you have a better } \\
\text { understanding than most. }\end{array}$ & im. + \\
\hline$C:$ & $\mathrm{p} 49$ & $\begin{array}{l}\text { I tend to look at the pedantry issue the other way round; } \\
\text { what place could it more appropriate or more necessary for } \\
\text { me to unleash my otherwise (slightly) restrained pedantry } \\
\text { than on a forum populated by students? [ } \backslash \text { ] I've long since } \\
\text { realised that it's pointless trying to represent myself ac- } \\
\text { curately on TSR so instead I find it a much more pleasant } \\
\text { (and less frustrating) use of my time to be far more con- } \\
\text { frontational and picky than I otherwise would, and pretend } \\
\text { that I'm some sort of wit. }\end{array}$ & im. $\varnothing$ \\
\hline$m:$ & p56 & $\begin{array}{l}\text { Of the two options, unleashing your pedantry upon real life, } \\
\text { or upon anonymous strangers in a student forum, I would } \\
\text { agree that the second option is better. Even so, its still not } \\
\text { appropriate or neccessary in most cases. Just annoying. Not } \\
\text { what people want to be distracted with, especially in light } \\
\text { hearted discussions, like this one. You can learn to recognise } \\
\text { when its appropriate to be pedantic, I've found. [ } \backslash] \text { People } \\
\text { will always have communication issues and not express } \\
\text { their views perfectly, or interpret questions differently. The } \\
\text { nature of language itself prevents perfect communication. } \\
\text { Just have to do your best to understand I guess. }\end{array}$ & $\operatorname{im} . \varnothing$ \\
\hline
\end{tabular}




\begin{tabular}{|l|l|l|l|}
\hline $7:$ & $\begin{array}{l}\mathrm{p} 42 \\
(\rightarrow 40)\end{array}$ & $\begin{array}{l}\text { You're always speaking the truth, everytime i read your } \\
\text { posts they're always quality either funny/clever/helpful @i }\end{array}$ & im. + \\
\hline C: & $\mathrm{p} 43$ & $\begin{array}{l}\text { Unfortunately evidence in this thread suggests that those } \\
\text { whose opinions matter the most (at least if I am to ever get } \\
\text { laid again) - the lady types - are not in agreement. }\end{array}$ & im. \\
\hline $7:$ & $\mathrm{p} 46$ & $\begin{array}{l}\text { I must agree then, your internet reputation has been } \\
\text { dented, you must hide all evidence of your existence in this } \\
\text { online world }\end{array}$ & im. $\varnothing$ \\
\hline
\end{tabular}

After the bonding between $m$ and $T$ in posts 22,26 and 38, the latter one also addresses two other users, $C$ and $A$. Naturally, the three different addressees elicit different types of interpersonal behavior, mirrored in the labels attached to this post: "im. +" and "ex. - r". In discussing C's behavior, the ensuing interaction between $m$ and $C$ also touches on the coherence of the thread and on what is appropriate in TSR and what is annoying. Unexpected help for C's face arrives in form of user 7 , who does positive facework for $C$ by praising the enduring quality of his posts.

Astonishingly, even insults can be subject to negotiation, as the following excerpt - despite being rather cryptic in terms of content - proves:

\begin{tabular}{|c|c|c|c|}
\hline$B:$ & p9 & No, it's very feckable. & im. $\varnothing$ \\
\hline$a:$ & $\mathrm{p} 45$ & $\begin{array}{l}\text { as far as i undestand, i'm assuming someone's written that } \\
\text { down in a sketchbook somewhere. or more, screenshotted } \\
\text { it and put it into a large word document entitled "I WILL } \\
\text { GET HER" }\end{array}$ & im. $\varnothing$ \\
\hline$B:$ & $\mathrm{p} 47$ & (9) Do you think someone cares that much? & im. $\varnothing$ \\
\hline$a:$ & p50 & $\begin{array}{l}\text { Well, I know nothing about TSR, however, I stumbled } \\
\text { across the poem thread for Cultivated which I think sug- } \\
\text { gested as much....? [ } \backslash \text { ] However, I'm just a little creeped out } \\
\text { that you don't find my comment rather insulting }\end{array}$ & im. $\varnothing$ \\
\hline$B:$ & p51 & Should I? [ $\backslash]$ Is it? & $\operatorname{im} . \varnothing$ \\
\hline$a:$ & p53 & $\begin{array}{l}\text { Well no, it just makes me out to be a lot more stalkerish } \\
\text { than I am... [ } \backslash] \text { Meh insulting was probably the wrong } \\
\text { word, but hey, most of my words are the wrong words... }\end{array}$ & im. $\varnothing$ \\
\hline$B:$ & p54 & I'm used to stalkers on here tbh. & $\operatorname{im} . \varnothing$ \\
\hline$a:$ & p55 & oh..... $\Theta[\backslash]$ and you're still here? & im. $\varnothing$ \\
\hline
\end{tabular}


Table 28. Relational work in thread \#13

\begin{tabular}{|c|c|c|c|c|c|c|}
\hline \multirow{2}{*}{\multicolumn{2}{|c|}{$\begin{array}{l}\text { Type of post } \\
\text { and evaluation }\end{array}$}} & \multicolumn{3}{|c|}{ Functional $\rightarrow$ appropriate } & \multicolumn{2}{|c|}{ Dysfunctional $\rightarrow$ inappropriate } \\
\hline & & marked + & marked + & unmarked & marked & marked - m \\
\hline \multirow[t]{2}{*}{ A: $30^{*}$} & ex. & 2 & 0 & & 1 & 0 \\
\hline & $\mathrm{im}$. & 2 & 0 & 24 & 1 & 0 \\
\hline $\mathrm{B}_{\mathrm{t}}: 32$ & $\mathrm{im}$. & 0 & 0 & 27 & $2(2 r)$ & 3 \\
\hline \multirow{2}{*}{\multicolumn{2}{|c|}{$\sum: 62(+1 \mathrm{C})$}} & 4 & 0 & 51 & $4(2 r)$ & 3 \\
\hline & & \multicolumn{3}{|c|}{55} & \multicolumn{2}{|c|}{$7(2 r)$} \\
\hline
\end{tabular}

* +2 exceptions p30: im. - r, im. $\varnothing$

p38: im. + , ex - r

Table 28 summarizes the relational work found in this thread. A look at the other 22 threads within this category reveals even more possibilities to more or less meet the expectations of the respective thread starters. In the following, these strategies will be listed and illustrated with a few examples.

As we have seen before, the initial question or problem contained in the thread start can be answered in more or less detail, ranging from a single smiley to several paragraphs. Some users do with a single quote to phrase their response, as in "Whoever kills himself, kills a man". $[\backslash]$ - St. Augustine (\#6p56). Other TSR-members make use of the multimodal potential of the communicative platform, for example by embedding links to other webpages (see, for example, \#3p62, \#27p5 or $\# 28$ p34, 73). These may include links to "You Tube"-videos (\#20p38) or any kind of picture to be found online (\#20p9). Then again, the latter two can also be directly embedded in the entry (\#7p97, \#20p21). The screenshot taken from thread \#32, post 6 illustrates how a picture can serve as a response almost single-handedly.

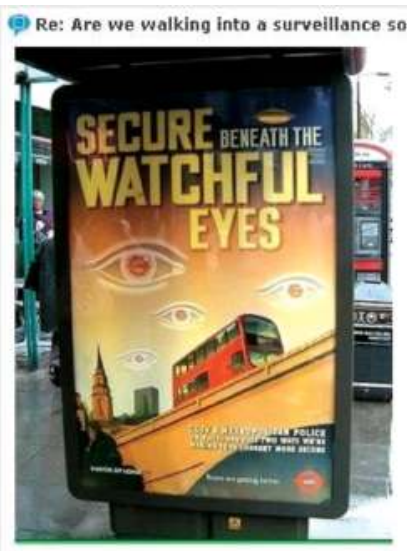

Too late!

In agreeing with certain lines of argumentation of their predecessors, participants do not necessarily have to be wordy to make their agreement known, but can resort to other, sometimes message board specific means of expression: 
Wow, I Agree Completely $\odot \odot[1]$

I second this.

snap!

(\#25p77)

yes.

Hear hear.

(\#50p56)

EXACTLY.

(\#8p21)

My thoughts exactly.

(\#7p18)

i concur!

(\#31p15)

[... reconfirmation of emma watsons utter perfection. :) ] DAYUMMM she's fine!! $\mathbf{\theta}_{\text {目 }}$

I know man!

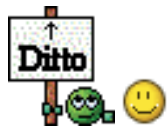

(\#20p10)

(2) [animated smileys high-fiving]

$\wedge$ This one.

(:) $[\backslash]$ This. This. A thousand times this! I don’t think I've ever [...]

(\#20p32)

As a look at the above list reveals, a lot can be expressed with the help of smileys, which cannot only be used to accompany and modify utterances but which can also serve as entire utterances by themselves: To signal agreement, the smiley in \#20p10 holds up a sign to refer to the previous entry, while \#7p24 shows two animated smileys which are about to high five. The same effect, referring and agreeing, is achieved by another strategy, registered towards the end of the list: using the determinative pronoun this, sometimes in combination with small arrows (see \#3p4) to indexically portend to the point made in the preceding entry.

On an interpersonal plane, this kind of verbal behavior involves bonding and sympathizing with others over shared views and experiences. While this is only implicitly contained in some of the above excerpts, it becomes explicit in the following examples:

I'm exactly the same. [I] OP, I don't think [...]

I feel awful for you

Same here and I'm $23 \oplus$ Birmingham and North Wales are the furthest North I've been. Never been to Scotland. 
I feel muchhh the same as you, OP. $\circledast[\backslash]$ I suppose $[\ldots]$

My feelings exactly. No wonder I didn't [...]

In this context, one should not forget to mention either that this also includes the sharing of emotions:

thinking about it makes me want to punch one right now punch something now. [...]

Grrr I need to

I am never happy thesedays. [ $\backslash$ ] I try to be happy, going out etc ... but I never reach a state where I can say "I am satisfied and happy with life" (Tis)

!!!ICE CREAM!!! [1] 涫紧

I love my grammar school (L)

Besides bonding, positive facework can also be accomplished by praising others' contributions explicitly, a strategy which mostly subsists on metapragmatic utterances:

Good argument

[...] You just pooned the Harry Potter heathen. Well done.

Good point.

brapboybrap's posts make me happy.

Then again some users go as far as distributing positive reputation for entries which were very much to their liking; some users at least promise to do so. Others, very aware of the possibility of being negatively "repped", give utterance to that fear also:

lol +rep

Awesome post, I'll rep you when I've replied $(\underset{*}{*}$

Ross and very close behind is Chancey (+rep for anyone who can name the episode where this comes up)

I just want to say that I agree wholeheartedly with missygeorgia. Positive rep for you. थี1

If you could, please PM me tomorrow so I can pos-rep you. I haven't got a rep left today.

[...] (awaits bad rep, but TBH, I don't care)

Who cares, we all know London is the best place to be in England. ( Please don't neg 
In this category, another kind of cooperative behavior is demonstrated by users who engage in the negotiation of meaning, be it in terms of clarifying and asking for clarification with regard to the phrasing of certain entries, reference or (potential) misunderstandings:

L: I think he means where is the $i \mathrm{i}$ am from the midlands and ... southerners /northerners'

d: Ooooooooooooooooooooooooooooooooooooooooooooooooooooh ...yeah, think you're right. Long day! probably a sign that i should be off to bed. tata TSR $:-$

W: Just take a look in H\&R.

$\mathrm{m}$ : I don't get it 8

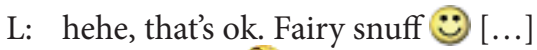

E: Fairy snuff?!

L: Fair enough

E: Lol! I'm stealing that!

P: It depends how you define the pay gap. [...]

E: Maybe you misunderstood me. I am not holding [...]

P: Yeah I was agreeing with you. The problem is [...]

E: I wasn't sure if you were agreeing or disagreeing lol, you said stuff that agreed with me but it seemed like you were disagreeing for some reason. (\#27p25)

E: That isn't the point I was making, could you at least read what I was replying to and not quote out of context? [...]

(\#27p41)

$\mathrm{m}$ : I was specifically responding to the how does somebody being a feminist in this country help women in Sudan?' comment, I should have made that clear.

Read my posts again, not once did I call you boring, that was another poster.

[...] I'm not sure what you mean. Could you clarify?

(\#29p89)

Well, I was addressing the OP more than you, but I suppose that it equally applies (as your last sentence certainly seems to imply a rule from what precedes, but I'll give you the benefit of the doubt). [...]

(\#37p29)

Especially thread starters are often faced with the need to revisit the phrasing of their original posts or their personal viewpoint of the topic conveyed in it. With a view to the following excerpts, all of which produced by the initiator of thread \#4, this can indeed turn into a challenging endeavor: 
can i stress again that $\mathrm{i}$ am not in favour of prohibition - i'm not an idiot - that clearly doesn't work - maybe my title was poorly thought through and people are not actually reading the post - bit long i suppose

did anyone actually read anything other than the title? [...]

again - not personally in favour of prohibition - which i said in my OP - and yes i know it doesn't work - i just titled my thread as such to catch people's attention/stimulate discussion - obviously wrong way to do it

(\#4p31)

Similar to previous categories, the dropping of lines as well as the negotiation of face claims can also be witnessed in this category:

I'm a Southerner and I'd definitely say Northerners. I'm not very friendly.

im still weirder though :'(

[...] I'm a genius for figuring that one out.

this will probably be an epic post. I am SUCH a geek. [...]

[...] OP sounds like a right cock. [...]

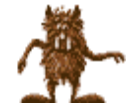

$[\backslash]$ The standard is really dropping these days ...

(\#28p64)

OP - Are you gay?

Being snug. I've discovered recently that I'm a very tactile person, and feeling very cosy, secure and safe makes me smile. $[\backslash]$ Sam $[\backslash] \mathrm{xxx}$

(\#33p23)

Metapragmatic utterances, as stated before, are an integral part of conversations, online and offline, and of the negotiation of relations therein, as they are often used as a lubricant to avoid anticipated friction. This can also be witnessed in the following excerpts in which users apologize for (parts of) their entries, for instance for their length, topic drifts or outbursts of emotions:

[...] Sorry if that turned into a bit of a rant.. erm.

[...] (Sorry if I went off topic)

Sorry double post

Sorry this post is so long and took such a long time to post; I just really wanted to try and give a decent answer $\because$

[...] Crazy. /end rant.

The same function, averting anticipated friction, can be assumed for the following set of examples in which participants point to or correct formal mistakes in their entries or make explicit the tentative nature of their opinions: 
Edited to correct grammatical error.

(\#20p62)

Can someone edit the title its supposed to be Creation OR evolution

oh ${ }^{* * *} \mathrm{i}$ think i made an error somewhere in that last post

Edit: Fail. Only just noticed there was a 2nd and 3rd page to this thread and that it wasn't a new thread.

(\#50p55)

[...] but that's just my opinion.

I know this is a stupid thing to say in a hypothetical situation, but [...] [ $\backslash]$ Sorry for the lack of sensible answers! 9

(\#10p10)

someone correct me if i'm wrong (i also haven't read the book in a few years)

kinda off topic but [...] (I stand to be corrected) [...] [ $]$ Apologies if this is a stupid question but i was wondering [...]

(\#30p38)

Of course, not every reaction is as positive or as constructive in terms of topical progression as the ones described so far. While some interlocutors simply disagree with previously posted views, others outspokenly state their disinterest for the entire topic. In doing so, users clearly set themselves apart from the rest of this particular community of practice, thereby attending to their need for dissociation. Others even go one step further by contributing ironic or joking, partly even offensive answers, which mock or insult either the thread starter or other users. The following list provides examples for all of these types of responses:

(1) disagreeing:

sorry, but i dont see how your coming up with these theories when youve admitted that $[\ldots]$

Lies.

Correction, they are not treated equal in some parts of society, [...]

Err, no.

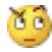

(2) stating disinterest:

cant say i give a flying monkeys

yeah, okay, whatever.

$\mathrm{Tl} ; \mathrm{dr}$. [= too long, didn't read] [ $\backslash]$ Is this a new subcontinent? Well it sounds lovely - I put "yes". [I] I kid, I'm not that stupid. [thread topic: Are you in favour of Euthanasia?]

(\#6p38) 
(3) answering ironically/jokingly:

Everybody should be watched, all the time... [ $\backslash]$ Well ok, poor people should be watched all the time, you just know that they're up to no good... $:$ [thread topic: Are we walking into a surveillance society?]

Fentanyl. [thread topic: What makes you happy?]

(4) mocking, replying offensively:

Are you ${ }^{\star * * * * * *}$ mad?!

So in your view, [...] [\] DaveSteed24 for Prime Minister!!!

[...] You see, I at this point like to use something called COMMON SENSE [originally: $36 \mathrm{pt}$ ], and i use big letters because its clearly something you are lacking... $[\backslash][\ldots]$ People like you is whats wrong with the world, interferring busy bodies who see other people [...] and finally, before your pea sized brain explodes, note that [...] [\] So there we go. Go take a half an asprin to ease that tired brain of yours..

(\#4p58)

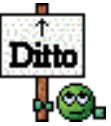

The OP [= opening poster] really didn't think this through

ahah you're so ignorant. I'm not even gonna bother

18 idiot.

Grow a pair OP, for god's sake. [...]

Stupid post.

Bugger you. [...]

Haha - well as you yourself demonstrate, grades clearly aren't everything, darling.

(\#37p13)

At this point, a selection of the most noteworthy passages regarding the management of interpersonal relations within this category and their evaluation shall be presented. In thread \#3, we encounter the following short exchange:

\begin{tabular}{|l|l|l|l|}
\hline$V:$ & $\mathrm{p} 3$ & Most people on here are social rejects. & ex. $-\mathrm{r}$ \\
\hline$G:$ & $\mathrm{p} 4$ & $\wedge$ This one. & $\mathrm{im} .-\mathrm{r}$ \\
\hline
\end{tabular}

What is interesting about these two utterances is the fact that by agreeing with the preceding entry, $G$ adopts not only the propositional content of $V$ 's entry but above that its interpersonal orientation, making his contribution as rude as that of his predecessor. (For the sake of completion, it should be noted that $V$ received its explicit, negative evaluation from another user.) 
A clear-cut case of rudeness thanks to the explicit evaluation by a fellowuser presented itself in thread \#7, in which the thread starter asks Who are more friendly: Northerners or Southerners (England):

\begin{tabular}{|l|l|l|l|}
\hline$h:$ & p113 & $\begin{array}{l}\text { tbh I think the northerners are just jealous that us southern- } \\
\text { ers are richer than them so whinge about how they smile } \\
\text { at each other in the street more (who the } \\
\text { happens????) to make up for their inferiority complex. [I] } \\
\text { Really they should be greatful - all regions of the UK spend } \\
\text { more in tax than they pay, apart from the south-east and } \\
\text { London, which pay more in tax than they spend. Basically, } \\
\text { the northerners suck on the south-east's juicy cock }\end{array}$ & ex. \\
\hline$O:$ & p114 & $\begin{array}{l}\text { you're really think it's an inferiority complex, and not, } \\
\text { y'know, rudeness like that? like in your post just then? }\end{array}$ & im. $\varnothing$ \\
\hline
\end{tabular}

Thread \#46 saw another strategy to handle anticipated negative reactions (see above), namely by means of something that could be labeled preemptive rudeness:

And the very fact I just said "l'art pour l'art" probably makes me pretentious. But I like the way my mind works now, it gives me satisfaction, so $\mathrm{Ct}_{3}$ really if you're going to criticise me for it.

Speaking of rudeness, thread \#4 (booze illegal?) clearly takes the cake with more instances of "ex. - r" (10 times) than any other thread in and outside of this category. This can mostly be blamed on the thread starter $D$, who adopts a very active role: In his 13 (out of 67) further contributions, he cannot seem to let pass any opportunity to pick a fight, which more often than not leads to a substantial flame. The following excerpt depicts only one of these cases:

\begin{tabular}{|c|c|c|c|}
\hline$D:$ & p12 & $\begin{array}{l}\text { and you suffer because you can't drink at home? - when it is } \\
\text { stll available elsewhere - how exactly? - excuse my boring } \\
\text { nature Mr Life of the Party }\end{array}$ & ex. $-\mathrm{m}$ \\
\hline$K:$ & p16 & $\begin{array}{l}\text { yea, thats it. You've solved our drinking problems right } \\
\text { there, with all the people who get stupidly drunk at home } \\
\text { every night? } 9[\ldots][\backslash][D] \text { for Prime Minister!!! () }\end{array}$ & ex. $-\mathrm{m}$ \\
\hline$D:$ & p17 & $\begin{array}{l}\text { clearly i've touched a nerve }-[\ldots]-\text { excuse me if my post } \\
\text { has somehow threatened your way of life - smart-arse }\end{array}$ & $\begin{array}{l}\text { ex. }-\mathrm{m} \\
\mathrm{ex} .-\mathrm{r}\end{array}$ \\
\hline
\end{tabular}




\begin{tabular}{|c|c|c|c|}
\hline$K:$ & p21 & $\begin{array}{l}\text { [...] Well, if your in a club, do you expect to be around the } \\
\text { local church group sipping on fresh orange and cups of } \\
\text { tea? No, its going to be drunk people, you don't like it, don't } \\
\text { go - I don't go to church, then complain about all the people } \\
\text { praying, or to a WI meeting, and complain that there is no } \\
\text { men - I'm sure you wouldn't be missed. [...] }\end{array}$ & ex. $-r$ \\
\hline$D:$ & p24 & $\begin{array}{l}\text { your really only enforcing the fact that } u \text { are a smart-arse - } \\
{[\ldots] \text { - the main point of my argument which all TSR's binge }} \\
\text { drinkers have conveniently side-stepped to mock me - i am } \\
\text { deeply sorry that my suggestion to make it only available } \\
\text { in pubs was so unutterably stupid - i am humble enough to } \\
\text { admit that this is not the solution - the point of this thread } \\
\text { was to discuss other solutions to the problem of binge } \\
\text { drinking - not to mock me and post sarky comments - i at } \\
\text { no point said that my solution was the correct one - obvi- } \\
\text { ously the words 'perhaps' and 'would this help?' fell on } \\
\text { deaf - smart-arse ears but there we are }\end{array}$ & ex. $-r$ \\
\hline$K:$ & p25 & $\begin{array}{l}\text { Well, perhaps I am a smartarse, but I would rather be a } \\
\text { smartarse than a going around suggesting stupid ideas, } \\
\text { that wouldn't work in a month of sundays. [ } \backslash \text { ] Also, your } \\
\text { OP didn't mention anything about other solutions to binge } \\
\text { drinking - [quote from the OP] Oh, and I know what a } \\
\text { luxury is (I'm a smart arse if you hadn't noticed (9)) and } \\
\text { driving is most definatly a luxury or have you never heard } \\
\text { of public transport up there on your high horse? }\end{array}$ & ex. $-r$ \\
\hline$D:$ & p29 & $\begin{array}{l}\text { well i was obviously misguided in thinking that some of the } \\
\text { users of this website who commented on my post might } \\
\text { actually be able to discuss something and voice their own } \\
\text { solutions by themselves rather than call people boring } \\
\text { because of an opinion but that's my mistake - }[\mathrm{I}] \text { and i } \\
\text { have obviously heard of public transport and while u say } \\
\text { there are other places to get around than cars i say there are } \\
\text { other ways to enjoy yourself than drinking - yes driving is } \\
\text { a luxury - but a necessary one to some people - i don't see } \\
\text { how drinking is necessary - but i'm sure you'll enlighten me } \\
\text { on that soon enough }\end{array}$ & ex. $-r$ \\
\hline
\end{tabular}




\begin{tabular}{|l|l|l|l|}
\hline K: & p48 & $\begin{array}{l}\text { Read my posts again, not once did I call you boring, that } \\
\text { was another poster. [\] Also, I see now, because I have } \\
\text { disagreed with your views, your calling me an alcoholic or } \\
\text { saying I need alcohol to enjoy myself. Neither is true, but } \\
\text { I'm sure that wont matter to you, because I am not enagag- } \\
\text { ing in your wonderfull discussion. }\end{array}$ & im. r \\
\hline$D:$ & $\mathrm{p} 49$ & oh - sorry about that $[\backslash]$ well stop posting then & im. $-\mathrm{m}$ \\
\hline
\end{tabular}

That there is indeed another way of handling these kinds of face threats is demonstrated by two other users in the same thread. Since $q$ does not reciprocate the offensiveness of L's remark, but chooses to agree with him instead, their discussion does not degenerate into a flame:

\begin{tabular}{|l|l|l|l|}
\hline q: & p30 & $\begin{array}{l}\text { Yup. Never really got binge drinking myself. I don't need } \\
\text { alcohol to be happy; sad most of our country does. Maybe } \\
\text { it's cos we live in such a dump? } 9\end{array}$ & im. \\
\hline$L:$ & p34 & $\begin{array}{l}\text { The rest of the country simply don't think in the same way } \\
\text { as you do, thank the Lord. [...] That said, you probably } \\
\text { didn't give very much thought to your statement, otherwise } \\
\text { you might have exposed the inherent nonsense in it without } \\
\text { my help. Chances are you just wanted to jump up on your } \\
\text { high-horse and look clever. Well, you've failed. }\end{array}$ & ex. \\
\hline$q:$ & p36 & $\begin{array}{l}\text { lol, you are right I don't care much hence my lack of word } \\
\text { on this page compared to yours. [I] It worries me you put } \\
\text { family in the same bracket as booze though. }\end{array}$ & im. $\varnothing$ \\
\hline$L:$ & p42 & $\begin{array}{l}\text { Both are ordinary parts of a normal person's life. My point } \\
\text { was that one's lifestyle is not divisible by parts, however } \\
\text { small. }\end{array}$ & im. $\varnothing$ \\
\hline
\end{tabular}

After a longer sequence of exchanging arguments, users in thread \#30 also decide to take the high road instead of turning the discussion into a flame:

Fair enough. Let's agree to differ lol. But I can't help commenting that it still doesn't allow for a 'more scientific creation of the earth'.

Worth mentioning in this context is a type C post to be found in thread \#27, as it provides insightful information about the perceived outcome of a previous flame - at least from the perspective of one co-participant of the two arguing parties: 
One last aspect that needs to be dealt with in this paragraph concerns those users that have been labeled as "do-gooders" and "trouble makers" in Section 7.3.2, since this category saw six out of twelve of these special thread starters in action. The behavior of the only "do-gooder" among them, MelonFace (\#46), which was observable in eleven further contributions apart from $\mathrm{p} 1$, was evaluated in nine cases as "im. Ø", while the other two entries earned the labels "ex. +" and "ex. -“. Hence, MelonFace $(M)$ has indeed proven to be a "do-gooder". Even his only misstep is corrected immediately:

\begin{tabular}{|c|c|c|c|}
\hline M: & p54 & $\begin{array}{l}\text { This doesn't really make any sense. But one is rather posh, } \\
\text { isn't one? }\end{array}$ & ex. - \\
\hline$B:$ & p55 & $\begin{array}{l}\text { oh ... } 98 \text { no, not posh at all. I'm sorry you didn't un- } \\
\text { derstand, but it's a bit unkind to make a comment like that } \\
\text { because you don't think I was clear enough. [ } \backslash] \text { I'll try to } \\
\text { simplify: I think that [...] }\end{array}$ & im. $\varnothing$ \\
\hline$h:$ & $\begin{array}{l}\mathrm{p} 56 \\
(\rightarrow 55)\end{array}$ & $\begin{array}{l}\text { Well, to be fair, there was a bit of verbal fluff in that post, } \\
\text { but I assume you were aware of that when you wrote it. }\end{array}$ & ex. - \\
\hline M: & $\begin{array}{l}\mathrm{p} 57 \\
(\rightarrow 55)\end{array}$ & $\begin{array}{l}\text { sorry, it does make sense, and its better than 'dat lit be gd } \\
\text { WUUUT' atleast, but i'm slightly hungover, and was too } \\
\text { tired to understand } 9 \text {. I think you would make quite a } \\
\text { good politician... }\end{array}$ & ex. + \\
\hline$B:$ & $\begin{array}{l}\mathrm{p} 58 \\
(\rightarrow 57 \\
56)\end{array}$ & $\begin{array}{l}\text { WI'm glad you called me on it anyway, because I some- } \\
\text { times get far too carried away with the intoxicating } \\
\text { elegance of a well-turned phrase ... And that's never fun to } \\
\text { plough through when you're hung over. }[\backslash] \text { I also agree that } \\
\text { 'dat lit be gd WUUUT'. } \\
\text { [quote h's contribution] } \\
\text { Fluff? Ouch. Overwritten, perhaps, but self-parody is the } \\
\text { only way for my crushing anxieties and excessive perfec- } \\
\text { tionism to be kept from silencing me entirely. [ } \backslash] \text { Gosh, all } \\
\text { my literary flaws are coming to light today, aren't they? I } \\
\text { think it's nap time. }\end{array}$ & im. $\varnothing$ \\
\hline$h:$ & p59 & $\begin{array}{l}\text { Well, "excessive polysyllabic verbiage which is super- as } \\
\text { well as mellifluous", then, if you prefer. I thought "fluff" was } \\
\text { a nicer way of putting it. * shrug* }\end{array}$ & im. + \\
\hline$B:$ & p60 & Metafluff! & $\operatorname{im} . \varnothing$ \\
\hline
\end{tabular}




\begin{tabular}{|l|l|l|l|}
\hline$h:$ & p61 & $\begin{array}{l}\text { Hmm, but wouldn't that be fluff which transcends the es- } \\
\text { sence of mere fluffness? }\end{array}$ & im. $\varnothing$ \\
\hline
\end{tabular}

In this exchange, the interaction between $h$ and $B$ is also noticeable: What seems to have been a well-phrased and appropriate post (p56), adorned with a series of mitigating devices such as "Well, to be fair", "I assume" and a winking smiley, still hurt B's feelings in $\mathrm{p} 58$, thus earning a negative evaluation.

Adonis' behavior has already been discussed (see exemplary thread \#13 above). Of the remaining four "trouble makers", Don_Scott (\#27) took a backseat in his thread with three out of 86 subsequent contributions and did not stir up any trouble. Although ShiVji (\#8) made himself similarly scarce, his three (out of 57) entries are marked by an outspoken and critical tone - obvious, for example, in p4: "anon fail 6 ". Then again Barz (\#29) adopted a very active role by authoring 25 of the next 99 entries. In so doing, he managed to stay on topic and reply factually, thus proving his reputation wrong.

The last candidate on the list to be discussed is $M r_{-} M a d(M)$, initiator of thread \#28, who totally lives up to his reputation with the challenging, even condescending and insulting topic Women Make Crappy Musicians. Full of offensive remarks concerning the "lack of genius" in women, this extraordinary thread start sets the course for the interpersonal conduct in the entire thread - predictably in a very negative way. One example shall suffice:

M: [list of female musicians] <------- [I] THEY ARE NOT MUSCIAL GENIUS YOU DUMB BITCH!! THEY ARE JUST AVERAGE ARTIST. [I] HOW DARE YOU.

$m$ : Fine if you disagree with my opinion, but calling me a dumb bitch? Too far. [I] You have no right whatsoever. [ $\backslash]$ Do you offend everyone you disagree with? [ $\backslash$ Go back to your hole or something, idiot.

Amazingly enough, though, there are still users who try to react to his statement seriously by putting forward factual arguments. Others try to ignore him (“ $\odot$ yeah, okay, whatever.", p12). Taking this as a piece of evidence, one could argue that behavior in message boards, contrary to its reputation, deserves to be seen in a more positive light, since users do not automatically jump on each and every bandwagon.

Another reaction to this kind of offensive behavior involves users in analyzing the thread starter's face claims and/or the nature of the thread as such:

I love people like you!! Those guys who are willing to spout controversial crap in the hope that one day they'll be noticed!! Well I hope that now you've got a few reactions, you can feel truly appreciated and go on with life, with the belief that someone, somewhere cares $: ;$ 
.. your ignorant rant is obviously for attention and i'm pretty sure you don't believe and/or don't understand what you're on about. [I] well done.. (p21)

This thread is just going to become a

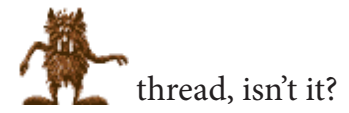

Yep, check out his rape convictions thread in H\&R [ $\backslash]$ Definite troll. Appears to be suffering from GIVEMEATTENTION-itis

Conclusively, the relational work of the entire fourth category can be depicted with the help of figures (see Table 29).

Similar to previous categories, Asking for opinions impresses with its display of 1670 cases of appropriate and functional behavior. With regard of the ratio of almost 15 to 1 cases of appropriate vs. inappropriate behavior, the overall impression of the fourth category can justifiably be characterized as harmonious, pointing to the fact that discussions in these 23 threads must have been - at least for the most part - factually. While nine posts were classified as type $\mathrm{C}$ posts and thus left out of the analysis and six entries received diverging evaluations (see exceptions), the remaining 111 contributions were labeled as inappropriate behavior. As seen before, more than half of these posts were not only impolite, but presented themselves as palpably rude. Accordingly, impolite posts - although rare when compared to the sum total of posts - are more often than not put forward unambiguously vehement.

Table 29. Relational work in the forth category (Asking for opinions)

\begin{tabular}{|c|c|c|c|c|c|}
\hline \multirow{2}{*}{$\begin{array}{l}\text { Type of post } \\
\text { and evaluation }\end{array}$} & \multicolumn{3}{|c|}{ Functional $\rightarrow$ appropriate } & \multicolumn{2}{|c|}{ Dysfunctional $\rightarrow$ inappropriate } \\
\hline & $\begin{array}{l}\text { marked + } \\
\text { "polite" }\end{array}$ & $\begin{array}{c}\text { marked + m } \\
\text { "mock-impolite" }\end{array}$ & $\begin{array}{c}\text { unmarked } \\
\varnothing\end{array}$ & $\begin{array}{l}\text { marked - } \\
\text { "impolite" } \\
\text { ("rude" = r) }\end{array}$ & $\begin{array}{l}\text { marked - m } \\
\text { "mock-polite" }\end{array}$ \\
\hline \multirow{2}{*}{ A: $\quad 719^{\star} \frac{\mathrm{ex} .}{\mathrm{im} .}$} & 25 & 0 & & $23(14 r)$ & 3 \\
\hline & 12 & 0 & 628 & $27(15 r)$ & 1 \\
\hline $\mathrm{B}_{\mathrm{t}}: 1062 \mathrm{im}$ & 17 & 0 & 988 & $48(28 r)$ & 9 \\
\hline \multirow[t]{2}{*}{$\sum: 1781(+9 \mathrm{C})$} & 54 & 0 & 1616 & $98(57 \mathrm{r})$ & 13 \\
\hline & \multicolumn{3}{|c|}{1670} & \multicolumn{2}{|c|}{$111(57 \mathrm{r})$} \\
\hline$*+6$ exceptions & $\begin{array}{l}\text { \#4p17: ex. } \\
\text { \#9p28: im. } \\
\text { \#10p110: im } \\
\text { \#13p30: im. } \\
\text { \#13p38: im. } \\
\text { \#28p1: im. }\end{array}$ & $\begin{array}{l}\text { r, ex. - m } \\
\text {, im. - r } \\
\varnothing, \text { im. }+\left(\text { post } B_{t}\right) \\
\varnothing, \text { im. - r } \\
+, \text { ex. }-r \\
\varnothing, \text { ex. }-r\end{array}$ & & & \\
\hline
\end{tabular}




\subsubsection{Blowing off steam}

This category comprehends threads which are used by TSR members to blow off some steam about a recent experience or a general problem. Although thread starters certainly do so in order to get responses from fellow-users -, why else go public with personal problems in a message board - they seldom outline their expectations for these responses. Accordingly, narrations end without questions being asked or advice being requested. There are hardly any guidelines for future interlocutors to follow, which is why, in theory, they enjoy absolute freedom in phrasing their contributions. In line with this first estimation, the figures describing the responses vary considerably: While the IQs are constantly medium (\#26: 37\%, \#12: 45\% and \#17: 48\%), the number of posts per day range between an above-average of $33 \mathrm{p} / \mathrm{d}$ for thread \#12 (I'm bitter and twisted), $11.5 \mathrm{p} / \mathrm{d}$ in thread \#17 (Burton's rejected me for a credit card, I think:S) and a below-average of $5.7 \mathrm{p} / \mathrm{d}$ in thread \#26 (Has anyone been to America recently ??... border control experience).

The thread to be discussed in detail in this category is \#12, as it elicited most responses per day and can thus be judged as the most attractive one among the three Blowing off steam-threads:

\section{I'm bitter and twisted}

Every time I go out I see fat people with friends, ugly people with friends, mean people with friends, nerds with friends, goths with friends, chavs with friends, bitches who used to bully me non-stop for all my school life - they all have friends. It makes me so angry. I want to hurt them. I have no friends, I have never had friends. [ $I$ ] I didn't used to be like this, I didn't care I just got on with it. But now I don't understand what's so ******* detestable about me that I'm incapable of making friends. Instead I get bullied, picked on and laughed at and randomly shouted at in the street and shunned from regular society like I'm some kind of total freak. [ $\mathrm{I}$ ] It's taken its toll on me. I see a psychiatrist, I take "medication", but it does sweet FA. I don't know what to do, I think it's jealousy eating me up, jealousy and lack of understanding - I don't understand how some people who all have their faults have friends but I don't. I didn't care before but as each day goes by I want to get back at the people who did this to me.

(Anonymous User \#1, thread \#12, post 1)

This is yet another thread to be initiated by the obscure participant Anonymous User \#1 (A) (see 7.3.2 and 8.2.3). This time, we are obviously dealing with a male person who is lamenting about his difficult (school) life and his incapacity to make friends, uttering the wish to get back at those he feels to be responsible for his mis- 
ery. He narrates his problem mainly with simple, sometimes complex declarative clauses without addressing anyone or asking for anything in particular. The only hint as to what this user is aiming at and what kind of response he might expect from his interlocutors can be found in the third paragraph: "I don't know what to do, $[. .$.$] " bears at least the potential to be read as an implicit request for advice.$

One way or another, this thread start prompted 35 mostly savvy users to author 65 responses, 14 of which were composed by $A$ himself, which hints to an active role of the thread starter. In trying to resolve misunderstandings, $A$ explains his viewpoint more than once, thereby keeping the conversation going. Very often, he does so by answering two interlocutors within one post, quoting and commenting on one after the other. Consequently, a tangled web of communicative threads is woven, which can only partly be reproduced in the following. A first overview can, however, be given with regard to the reactions of his co-participants, who basically engage in a lively and more often than not dialogic, partly even polylogic discussion about $A$ 's character and his problems. In fact, reactions vary considerably in terms of interpersonal appropriateness:

(1) neutrally analyzing the problem, e.g.

I believe jesus once said when he was laying down the rap to his homedogs: Do not fret about the mote in your neighbours eye before dealing with the huge goddamn stick up your ass. [ $\backslash$ In other words, you seem to be refusing to acknowledge your faults, and because of this you can't get rid of them and move on and get friends.

(2) giving of advice, e.g.

You need to find people you have common interests with (in films, music, literature, uni, sports and the list goes on), and be kind to them. That's it. [ 1$]$ As long as you act nicely and don't seem desperate, you should be able to make friends.

(3) sympathizing, e.g.

I can totally relate to you anon! It may seem unbelievable but you're not alone. I've travelled places, been outgoing, went to a london uni, [...] [ $\backslash]$ You're not alone.

(4) offering friendship, e.g.

Why should the teacher tell them that ? Quite a strange teacher 89. [ I] I'll be your friend,is that ok?

(5) putting negative self-perceptions into perspective, e.g. $[\ldots]$ Other than those very strange comments about revenge you don't seem too bad. 
(6) humorous comments, e.g.

Just go out more...into daylight!

(7) blaming him for his situation and criticizing his attitude, e.g.

You have no friends because you're bitter and twisted. [I] STOP HATIN' YO"

While all these reactions can be considered more or less helpful but in any case mostly appropriate (see $\mathrm{p} 4$ for a borderline case), other types of responses can be detected in which users mock and offend the thread starter, thus doubtlessly crossing the line to inappropriate behavior:

see what,you can not even find a mind-liked here,you thought everyone was misunderstanding you,then what the hell are you thinking about? maybe it's the poor ability of expression that makes you own 0 friend.ie when you say "may i make a friend with you?" [ $\backslash]$ peple may get the informationas this," you have a problem?" [I] sorry,i am no offence.

As the following excerpts attest, some of the most extreme responses do not stay uncommented. Interestingly, the thread starter seems to dwell on this kind of behavior, challenging his interlocutor to further elucidate his views:

\begin{tabular}{|l|l|l|l|}
\hline K: & $\mathrm{p} 48$ & $\begin{array}{l}\text { [quote: But now I don't understand what's so } \\
\text { able about me that I'm incapable of making friends.] Read } \\
\text { your intro.. That should answer it.. }\end{array}$ & ex. - r \\
\hline A: & $\mathrm{p} 49$ & $\begin{array}{l}\text { Go on then, what? [\] Bearing in mind I've already ex- } \\
\text { plained I don't view the various attributes I listed as faults } \\
\text { just that other people might and so how do these people } \\
\text { have friends. }\end{array}$ & im. $\varnothing$ \\
\hline K: & $\mathrm{p} 61$ & $\begin{array}{l}\text { Well you wondered why you don't have any friends? [\] } \\
\text { With that kind of attitude (referring to your intro) .. You're } \\
\text { not going to have any.. }\end{array}$ & im. - r \\
\hline A: & p62 & $\begin{array}{l}\text { You still haven't explained what kind of "attitude" you're on } \\
\text { about. }\end{array}$ & im. $\varnothing$ \\
\hline
\end{tabular}

In this extremely rude reaction of $I$ towards the thread starter $A$, another participant, $x$, leaps at the thread starter's defense. Although commenting on I's post, $x$ obviously addresses $A$, thus carrying out two types of relational work within one entry: 


\begin{tabular}{|c|c|c|c|}
\hline$I:$ & p10 & $\begin{array}{l}\text { go top yourself...it's the only form of productivity you can } \\
\text { provide to this world... } \oplus\end{array}$ & ex. $-r$ \\
\hline$x:$ & P41 & $\begin{array}{l}\text { seriously, ignore idiots like this seriously } \odot[\backslash] \text { and as for } \\
\text { what you said hun, i can see how it seems not fair!! but to } \\
\text { all the people that have bullied you etc... what goes around } \\
\text { comes around, just remember that } \odot \text { people should be } \\
\text { afraid of karma, because it will get them one day. [ }[\backslash \text { if } \\
\text { you've got no friends, maybe people pick up on your feel- } \\
\text { ings and deep inside you might feel so angry that its hard } \\
\text { for you to let go. instead, forget about the past... have a } \\
\text { fresh start. put on a smile, be friendly, and try and be more } \\
\text { social/go to social activities } ;\end{array}$ & im. + \\
\hline
\end{tabular}

Then again, some fellow-members gang up on the thread starter, stating their doubts about the truthfulness of the introductory post very bluntly:

\begin{tabular}{|l|l|l|l|}
\hline a: & p33 & $\begin{array}{l}\text { I'm sure you're exaggerating. Why on earth would random } \\
\text { people across the street make the effort to insult you? (Un- } \\
\text { less you're extremely odd looking.) }\end{array}$ & im. $\varnothing$ \\
\hline A: & p34 & $\begin{array}{l}\text { I promise you I'm not exaggerating. I didn't say across the } \\
\text { street, generally it's people on the same side of the street } \\
\text { shouting } \\
\text { my*** at me. Not sure if I'm odd looking, I asked } \\
\text { have to say that. I'm not white-British and was always bul- } \\
\text { lied for that. Might have something to do with it. }\end{array}$ & im. $\varnothing$ \\
\hline$W:$ & $\begin{array}{l}\text { p47 } \\
(\rightarrow 33)\end{array}$ & $\begin{array}{l}\text { i am totally on board with you! it seems the rest are all } \\
\text { freaks except himself. }\end{array}$ & im. \\
\hline
\end{tabular}

One last note on the questionable background of the thread starter: In looking at his reactions, one cannot help but wonder if the thread starter is seriously naïve and in need of help or rather playing his interlocutors for a fool. Though he is very quick at rephrasing ambiguous content over and over, he does not seem to show any interest when friendship (see p17) or sympathy (see p16) are generously offered. Ironically, the latter is taken up by somebody else and serves as a springboard for a mostly smiley-based but nevertheless empathetic exchange between $S$ and $L$ : 


\begin{tabular}{|c|c|c|c|}
\hline$S:$ & p16 & $\begin{array}{l}\text { I can totally relate to you }[A] \text { ! It may seem unbelievable but } \\
\text { you're not alone. I've travelled places, been outgoing, went } \\
\text { to a london uni, stayed in the social scene that is leicester } \\
\text { square till } 6 \text { in the morning trying to find } 1 \text { friend, someone } \\
\text { who will say they need a friend and wants to hang out. Even } \\
\text { I didnt care who it was, I was willing to chat to anyone ... } \\
\text { but you know what I ended up with? Ended up with chat- } \\
\text { ting to beggars on the street, and they are the most friendli- } \\
\text { est non-fake people you'll ever see. Theres nothing wrong } \\
\text { with me, I just think Im an omen or something to friend- } \\
\text { ship. }[\backslash] \text { You're not alone. }\end{array}$ & im. + \\
\hline$L:$ & p36 & (iii) & ex. + \\
\hline$S:$ & p38 & thanks so much $\mathrm{x}$ means alot $1 \mathrm{O}$ & ex. + \\
\hline$L:$ & p64 & $\begin{array}{l}\text { haha aww no problem! }[\backslash] \text { you will find great friends } \\
\text { soon enough }(:)\end{array}$ & $\operatorname{im} . \varnothing$ \\
\hline
\end{tabular}

The impression of $A$ being a fraud is further substantiated in the last example, in which, again, $A$ does not react at all to the advice given in $M$ 's post but to the typo instead. Consequently, $M$ is not heard of anymore in this thread:

\begin{tabular}{|c|c|c|c|}
\hline M: & $\mathrm{p} 21$ & $\begin{array}{l}\text { I think your exadurating. There must be something pretty } \\
\text { wrong if people are calling you out on the street now } \\
\text { wouldnt there? }[\backslash] \text { Go put some drum n bass music on } \\
\text { really loud and ride your bike down a road really fast and } \\
\text { disperse your rage into how cool you feel while your speed- } \\
\text { ing down the street. Then speak to people while your on an } \\
\text { ego-trip from your awesomeness. At these times conversa- } \\
\text { tion flows the best and your bound to make friends. [ } \backslash] \text { ? }\end{array}$ & im. $\varnothing$ \\
\hline A: & $\mathrm{p} 27$ & $\begin{array}{l}\text { What does exadurating mean? Google says it's not a word. } \\
\text { [ } \backslash \text { I don't know what's wrong with me, if I did I'd change it, } \\
\text { but I get a lot of comments from people when I'm just walk- } \\
\text { ing down the street it just makes me hate them even more I } \\
\text { can't believe people are that evil. }\end{array}$ & im. $\varnothing$ \\
\hline
\end{tabular}

Although this particular thread-initial post was received and reacted to quite heterogeneously, all 25 subsequent posts directly referring to said thread start judged it implicitly as appropriate (“im. Ø”). With this information in mind, the final account of relational work in this thread looks as follows (see Table 30): 
Table 30. Relational work in thread \#12

\begin{tabular}{|c|c|c|c|c|c|c|}
\hline \multirow{2}{*}{\multicolumn{2}{|c|}{$\begin{array}{l}\text { Type of post } \\
\text { and evaluation }\end{array}$}} & \multicolumn{3}{|c|}{ Functional $\rightarrow$ appropriate } & \multicolumn{2}{|c|}{ Dysfunctional $\rightarrow$ inappropriate } \\
\hline & & marked + & marked + & unmarked & marked & marked - m \\
\hline \multirow[t]{2}{*}{ A: 36} & ex. & 5 & 0 & & $2(2 r)$ & 0 \\
\hline & $\mathrm{im}$. & 2 & 0 & 26 & 1 & 0 \\
\hline $\mathrm{B}_{\mathrm{t}}: \quad 30$ & im. & 6 & 1 & 21 & 2 & 0 \\
\hline \multirow{2}{*}{\multicolumn{2}{|c|}{$\sum: \quad 66$}} & 13 & 1 & 47 & $5(2 r)$ & 0 \\
\hline & & \multicolumn{3}{|c|}{61} & \multicolumn{2}{|c|}{$5(2 r)$} \\
\hline
\end{tabular}

Despite some hostile and definitely inappropriate undertones, the bulk of the communicative exchanges (61 to 5 ) in this thread were nevertheless characterized by appropriate, sometimes even positively marked, polite behavior. In the remaining of this chapter, the two threads left to analyze for this category will be dealt with very briefly.

\section{Burton's rejected me for a credit card, I think :S}

Ok so I am paying for my clothes and she goes if you get a burton card you get $10 \%$ off on every purchase. I asked if it's free to get the card and how long it will take. [ $\backslash]$ She said it's free and will take 5 mins. [ $\backslash]$ Anyway on the phone she said it hasn't gone through, apparently they couldn't hear her very well $:-$ [I] Then I get a leaflet saying reasons for being declined a credit card. Wtf I don't have any CCJ's or anything. I think maybe cause I put my income as zero being a student? [ $\backslash]$ Anyway this is a rant, I'll be a dentist in a few years and that should settle it. [ I] I find it so stupid how they cannot say your aplication has been rejected, they always pussyfoot around the subject. Then I saw the same girl at a bus stop with fag in hand.

(Neo Con, thread \#17, post 1)

The profile and other contextual clues reveal this thread starter to be a US-American user who is known for his sense of belligerence, earning him an entry in the list of "trouble makers" (see Section 7.3.2). Living up to his reputation, he uses this thread opening to fume about being declined a credit card. In the course of the thread, however, Neo Con $(N)$ is very responsive, contributes actively ( 8 out of 22 reactions to the thread start) and shows himself - contrary to his negative reputation - thankful and relieved for the enlightening comments:

Thanks, I feel alot better now that you have said it could be because I put my income as zero. For awhile I panicked that maybe something is wrong with 
my history, but I haven't done anything so it couldn't be that. [ I] Yes I guess people do get abusive, I just don't see why someone would. I mean it's just burton's right lol?

Hmm now it's making sense. I always thought not having a credit history would make me clean but clearly you need to build it up like from banks as you say. [ 1$]$ Thanks for the help.

The otherwise uneventful and short thread involves interlocutors who are not directly addressed and whose reactions are not predetermined in any way, in speculating about reasons for this kind of treatment and sharing experiences about credit cards. The smooth and relaxed nature of this thread is also mirrored in figures (see Table 31).

\section{Has anyone been to America recently ??...border control experience}

I love the country $n$ all but taking your fingerprints and webcam snap...? Through border control. Thats kind of violation. They did it with everyone and $i$ went New York and i can understand that because of 9/11 and wanting to protect the country. Is that the case of other parts of America when entering.. $[\backslash]$ I found it very James Bond 007. It wasnt just one fingerprint it was both hands ..Just found it over the top.. I think other people felt violated too.. $[\backslash]$ And the border control guy was nice but the questions they have to ask like ..have you been to the states before/when was the last time you came? and whats the reason for your trip??...Lifestory or what..but again they did it to everyone

(Jesspops, thread \#26, post 1)

This relatively new member, Jesspops $(J)$, vents and wonders about airport procedures in the United States. Her introductory question in the heading can be regarded as a rhetorical one, which might, however, also serve as a springboard

Table 31. Relational work in thread \#17

\begin{tabular}{|c|c|c|c|c|c|c|}
\hline \multirow{2}{*}{\multicolumn{2}{|c|}{$\begin{array}{l}\text { Type of post } \\
\text { and evaluation }\end{array}$}} & \multicolumn{3}{|c|}{ Functional $\rightarrow$ appropriate } & \multicolumn{2}{|c|}{ Dysfunctional $\rightarrow$ inappropriate } \\
\hline & & marked + & marked + m & unmarked & marked - & marked - m \\
\hline \multirow[t]{2}{*}{ A: 13} & ex. & 2 & 0 & & 0 & 0 \\
\hline & im. & 0 & 0 & 11 & 0 & 0 \\
\hline $\mathrm{B}_{\mathrm{t}}: \quad 10$ & $\mathrm{im}$. & 0 & 0 & 10 & 0 & 0 \\
\hline \multirow[t]{2}{*}{$\sum: \quad 23$} & & 2 & 0 & 21 & 0 & 0 \\
\hline & & \multicolumn{3}{|c|}{23} & \multicolumn{2}{|c|}{0} \\
\hline
\end{tabular}


for other users' responses. Other than that, there is no further attempt to make contact with potential interlocutors, as her first post only contains a narration and evaluation of her experience. Although her subject yielded 85 responses, $J$ adopts a rather passive role and allows other users to run her thread, replying only three times after her initial post. Despite the flat hierarchy, some dialogic structures develop here and there.

In responding to the initial post, and very similar to the preceding thread, $\int$ s fellow-users share their feelings about border control and sympathize with her, sometimes even emphatically:

I hate American border control with a passion - rude to the point of offensive and when they decided to search my bags they dropped my camera (completely destroyed it) and apparently I was liable for the damage done!!! [ $]$ There is also one particular security officer at my closest British airport (deals with transition to air side) who seriously gets off on her job...I can accept that you have a job to do, but there is a difference between a frisk search and you ******* groping me!

Yeah, we kind of tend to be a bit crazy about border control in America, especially lately. It's kind of ridiculous after a certain point.

A lot of users are prompted to remember and share their own border control experiences, occasionally in some detail. Then again others relativize her negative feelings by means of finding arguments for the procedures or by downplaying the matter, sometimes with the help of humor:

Heh, it's so much worse when you're trying to enter America with an Arabic/ Muslim name like me. I got pulled into a room and had to prove I was there to visit etc.

It's happened for years, it's not that much of a big deal, if you're used to traveling.

I would agree that it could be a very stressful and intimidating experience, but you must bear in mind why they're doing it. It's nothing personal at all, it's simply attempting to help protect the lives of everyone in the country. They may come across as ignorant and rude, but that's simply a technique they use to put you under pressure so that if you are lying about something, you might crack and slip up on some facts, giving them reason to detain you further and gather more information. [...]

The explosive nature of this topic finds expression in two longish dialogic structures between two males and two females respectively, which can justifiably be called flames: 


\begin{tabular}{|c|c|c|c|}
\hline$d:$ & p6 & $\begin{array}{l}\text { At least we don't having CCTV everywhere we go! Nor do } \\
\text { we have an overbearing government, Americans just don't } \\
\text { want criminals entering our borders I guess. }\end{array}$ & im. $\varnothing$ \\
\hline G: & p17 & $\begin{array}{l}\text { [...] [quote: At least we don't having CCTV everywhere } \\
\text { we go! ] Neither does the UK. You believe everything you } \\
\text { read in the papers? [quote: Nor do we have an overbearing } \\
\text { government] LOL }\end{array}$ & ex. - \\
\hline$d:$ & $\mathrm{p} 23$ & 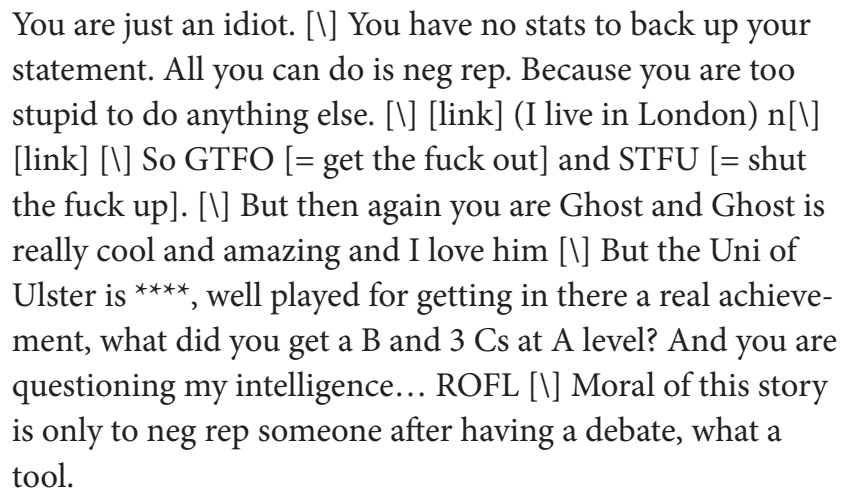 & ex. $-r$ \\
\hline G: & $\mathrm{p} 25$ & $\begin{array}{l}\text { Hahah, typical yank. You got so annoyed by my post that } \\
\text { you felt the need to rummage through my site? Nice one. } \\
\text { I'd like to think I've done fairly well for myself, not only did } \\
\text { I "get in there", but I also got a 1st class honours, and have } \\
\text { been awarded best graduate, and been accepted for a PhD. } \\
\text { If you could live up to even half of that, I'd be impressed. } \\
\text { [ } \backslash \text { Editing your post } 3 \text { times because you're THAT slow a } \\
\text { thinker is a bit pathetic. Go eat some twinkies, it'll make } \\
\text { you feel better. }\end{array}$ & ex. $-r$ \\
\hline$d:$ & $\mathrm{p} 28$ & $\begin{array}{l}\text { Lol all at the university of Ulster - very impressive... Sup- } \\
\text { posedly you need at least a C in math GCSE for comp sci } \\
\text { there. ROFL }[\backslash] \text { Actually we like to cure the problems of } \\
\text { Ireland and Northern Ireland, so your welcome. }\end{array}$ & im. $-\mathrm{r}$ \\
\hline$d:$ & $\mathrm{p} 26$ & $\begin{array}{l}\text { [quote: The only bit in your post that didn't make me cringe. } \\
\text { Keep it up. (Erased in the original post)] Wow you can read. } \\
\text { Well done. }\end{array}$ & ex. $-\mathrm{m}$ \\
\hline$D:$ & $\mathrm{p} 27$ & Awwwww is David getting angry? & ex. $-\mathrm{m}$ \\
\hline$d:$ & p29 & $\begin{array}{l}\text { Lol i was going to get angry with you but then I saw your } \\
\text { afro, sick afro man. }\end{array}$ & ex. $-r$ \\
\hline
\end{tabular}




\begin{tabular}{|l|l|l|l|}
\hline$r:$ & $\mathrm{p} 30$ & $\begin{array}{l}\text { Ffs }[=\text { for freak's sake] quit with the ad hominems and stick } \\
\text { to the point. }\end{array}$ & im. $\varnothing$ \\
\hline$D:$ & $\mathrm{p} 47$ & I am not here to fight, only here to make peace =P & im. $\varnothing$ \\
\hline$d:$ & $\mathrm{p} 49$ & $\begin{array}{l}\text { I also forgot to mention that I saw the janitor in your sig, } \\
\text { classic. }\end{array}$ & im. $\varnothing$ \\
\hline
\end{tabular}

The flame war between $d$ and $G$ is not only overt when looking at the responses of these two interlocutors, which contain blunt, off-topic insults and swear words in acronyms. Even more valuable for our analysis are the reactions of two other participants, $D$ and $r$, who try to intervene in two very different ways: While $D$ mocks $d$ in p27 only to clarify that he is not looking for any trouble in p47, realizing that $d$ is only too willing to take him on also, $r$ calls it as he sees it in p30 and urges them to stop with the ad hominem attacks, thereby reminding them of the TSR code of conduct. Since $G$ has already bowed out of the conversation, there is only $D$ left to deal with. In the end, $d$ decides to make peace with him by applauding part of D's signature ("the janitor" is a "classic"). ${ }^{76}$

A rather humorous reaction is taken as the springboard for the female flame war in this thread:

\begin{tabular}{|l|l|l|l|}
\hline Q: & p51 & $\begin{array}{l}\text { lol my fave bit is the green card questions they ask, as if } \\
\text { you'd ever say yes to any of them- i particularly LOL at the } \\
\text { nazi party participation "have you been a member of the } \\
\text { nazi party between } 198 \text { and 1939" or something like that. }[\backslash] \\
\text { "er no, I'm a 17 year old black girl." [ } \backslash]\end{array}$ & im. $\emptyset$ \\
\hline
\end{tabular}

\section{$[\ldots]$}

\begin{tabular}{|l|l|l|l|}
\hline$l:$ & p70 & $\begin{array}{l}\text { I would hazard a guess than more than 99\% of visitors to } \\
\text { the UK haven't committed genocide, either. [I] Why does it } \\
\text { bother you so much that there is a question on a form that } \\
\text { doesn't apply to you or most people? Get a grip. }\end{array}$ & ex. - \\
\hline
\end{tabular}

76. Common ground is created with reference to US pop culture: The janitor is a hugely popular character from the TV-series Scrubs. Embedding his picture in a signature is obviously met with approval. 


\begin{tabular}{|c|c|c|c|}
\hline$L:$ & p72 & $\begin{array}{l}\text { Really? There have been a hell of a lot of genocides within } \\
\text { living memory, and all it asks is if you partook in any shape } \\
\text { or form. I'd say it was more likely someone would fit that } \\
\text { criteria, than them having been a member of a specific fas- } \\
\text { cist government that hasn't existed for sixty years. But if you } \\
\text { think there are more } 80+\text { Nazis out there wanting to fly to } \\
\text { America to do their Nazi business, than there are relatively } \\
\text { young ex-soldiers from recent genocidal regimes who bear } \\
\text { a grudge, then more power to you } \Theta \text { lo } 9 \text { quote previous } \\
\text { post] It doesn't bother me at all, other than amusing me } \\
\text { with its sheer ridiculous nature. All that bothered me was } \\
\text { that you thought such an idiotic and amusing question was } \\
\text { the same as asking about any known terrorist affiliation, as } \\
\text { if you don't ask all those questions on top of the stupid Nazi } \\
\text { one. Why does it bother you so much that we think this } \\
\text { question is so idiotic? It's a dumb question, we're allowed to } \\
\text { be amused by it. Get a grip. }\end{array}$ & ex. - \\
\hline$l:$ & p74 & $\begin{array}{l}\text { You are getting way too worked up over this question. Take } \\
\text { a deep breath. }\end{array}$ & ex. - \\
\hline$L:$ & p75 & $\begin{array}{l}\text { I'm really not. I just thought I'd point out you're being } \\
\text { unreasonable. The question makes me laugh. You make me } \\
\text { facepalm. Nevermind though. }\end{array}$ & ex. - \\
\hline$l:$ & p76 & $\begin{array}{l}\text { You've written } 455 \text { words on why that question shouldn't be } \\
\text { on that application form. Complete fail. }\end{array}$ & ex. $-r$ \\
\hline$L:$ & p79 & $\begin{array}{l}\text { You cared enough about my posts to have the words in them } \\
\text { counted. I'd say that's more of a fail. }\end{array}$ & im. $-r$ \\
\hline
\end{tabular}

In this flame, the decline of their conduct from factual and topic-driven to personal and topic-unrelated is gradual. Since there is no bystander to thwart their destructive behavior, the flame only stops with both sides coming to a stalemate in terms of insults. In figures, the relational work in this thread can be summarized in Table 32. Although appropriate behavior is again superior to inappropriate behavior with 69 to 15 posts, there are some indicators for the heated exchanges elicited by users' controversial views on the topic: nine explicit cases of negatively marked and above that even rude behavior in addition to three other posts, which could at least implicitly be labeled rude. These stand in marked contrast to only one incidence of (explicit) politeness.

The entire fifth category, Blowing off steam, saw rather heterogeneous topics: an unhappy student in search of friends, an angry student who did not get a credit card and a shocked student whose report about border controls in the USA 
Table 32. Relational work in thread \#26

\begin{tabular}{|c|c|c|c|c|c|c|}
\hline \multirow{2}{*}{\multicolumn{2}{|c|}{$\begin{array}{l}\text { Type of post } \\
\text { and evaluation }\end{array}$}} & \multicolumn{3}{|c|}{ Functional $\rightarrow$ appropriate } & \multicolumn{2}{|c|}{ Dysfunctional $\rightarrow$ inappropriate } \\
\hline & & marked + & marked $+\mathrm{m}$ & unmarked & marked - & marked - m \\
\hline \multirow[t]{2}{*}{ A: 40} & ex. & 1 & 0 & & $9(4 r)$ & 2 \\
\hline & im. & 0 & 0 & 27 & $1(1 r)$ & 0 \\
\hline $\mathrm{B}_{\mathrm{t}}: 44$ & im. & 0 & 0 & 41 & $2(2 r)$ & 1 \\
\hline \multirow{2}{*}{\multicolumn{2}{|c|}{$\sum: 84(+2 \mathrm{C})$}} & 1 & 0 & 68 & $12(7 r)$ & 3 \\
\hline & & \multicolumn{3}{|c|}{69} & \multicolumn{2}{|c|}{$15(7 \mathrm{r})$} \\
\hline
\end{tabular}

Table 33. Relational work in the fifth category (Blowing off steam)

\begin{tabular}{|c|c|c|c|c|c|c|}
\hline \multirow{2}{*}{\multicolumn{2}{|c|}{$\begin{array}{l}\text { Type of post } \\
\text { and evaluation }\end{array}$}} & \multicolumn{3}{|c|}{ Functional $\rightarrow$ appropriate } & \multicolumn{2}{|c|}{ Dysfunctional $\rightarrow$ inappropriate } \\
\hline & & $\begin{array}{l}\text { marked + } \\
\text { "polite" }\end{array}$ & $\begin{array}{c}\text { marked }+\mathrm{m} \\
\text { "mock-impolite" }\end{array}$ & $\begin{array}{c}\text { unmarked } \\
\varnothing\end{array}$ & $\begin{array}{l}\text { marked - } \\
\text { "impolite" } \\
\text { ("rude" = r) }\end{array}$ & $\begin{array}{l}\text { marked - m } \\
\text { "mock-polite" }\end{array}$ \\
\hline \multirow[t]{2}{*}{ A: 89} & ex. & 8 & 0 & & $11(6 r)$ & 2 \\
\hline & $\mathrm{im}$. & 2 & 0 & 64 & $2(1 \mathrm{r})$ & 0 \\
\hline $\mathrm{B}_{\mathrm{t}}: 84$ & $\mathrm{im}$. & 6 & 1 & 72 & $4(2 r)$ & 1 \\
\hline \multirow{2}{*}{\multicolumn{2}{|c|}{$\sum: 173(+2 \mathrm{C})$}} & 16 & 1 & 136 & $17(9 \mathrm{r})$ & 3 \\
\hline & & \multicolumn{3}{|c|}{153} & \multicolumn{2}{|c|}{$20(9 r)$} \\
\hline
\end{tabular}

triggered the most diverse reactions. In figures these are portrayed in Table 33. Obviously, the main part of interpersonal exchanges of the three threads belonging to this category was characterized by appropriate (87\%) rather than inappropriate behavior (153 to 20 posts). Similar to previous categories, the amount of polite behavior is almost insignificant when compared to that of unmarked behavior (16 to 136), with mock-impoliteness as a special case of appropriate behavior being detected only once in 175 posts. On the other side of the scale, we can put on record that 9 out of 17 cases of impoliteness could also be labeled as rude. This hints at the fact that dysfunctional behavior is more often than not the result of a hurtful intention than it is accidentally.

In terms of facework, users' strategies towards their interlocutors, which have been mentioned above in some detail, can be grouped and summarized accordingly: In order to support each other's need for association, interlocutors analyzed problems and situations, gave advice, sympathized with each other, shared experiences and feelings, offered friendship, tried to raise the other's spirits (for 
example with humorous remarks) and showed themselves thankful. The second component of positive facework, viz. the support of the need for dissociation, was most of all achieved by accepting diverging opinions. On the other hand, we could also encounter cases of negative facework, in which the two basic human needs were not taken care of. In this context, we witnessed users putting others' views and problems in perspective by relativizing or even downplaying it, laying the blame for something on each other, verbally excluding someone from the community, doubting the truthfulness of someone's story or even mocking and flaming someone.

\subsubsection{Offering advice/information}

Despite the fact that interaction is not necessarily a must for a category, in which one savvy user offers advice and/or helpful information to whomever interested without being asked for it, we can find all types of IQs in the four examples of this category: a low IQ in thread \#36 (Guide to How to Revise Effectively?), a medium IQ in thread \#5 (Meteor Shower :)) and \#38 (Jolly useful Geography websites) and a high one in thread \#48 (QঊA thread - Journalism Courses). All four of them share, however, a below-average amount of posts per day, which is in two cases even below $0.1 \mathrm{p} / \mathrm{d}$ (\#36: 0,09 p/d, \#38: $0.05 \mathrm{p} / \mathrm{d}$ ). Thread \#48 exhibits at least an amount of $3.1 \mathrm{p} / \mathrm{d}$, \#5 even $10.6 \mathrm{p} / \mathrm{d}$. Obviously, the four initial posts in this category do not contain content and/or are not phrased in a way that prompts others to answer immediately, let alone numerously. To illustrate this category, thread $\# 48$ as the one scoring highest in terms of IQ will be chosen as an example:

\section{Q\&A thread - Journalism Courses}

$\mathrm{Hi},[\mathrm{I}]$ Everyone seems to be asking the same questions so I thought it would be easiest to set up a Q\&A thread about Journalism courses here. I will do my best not to be biased and will answer any questions as best I can. [ I] [Q\&A section] [ $\backslash]$ Hope this helps. Feel free to ask any more questions $: ;[\backslash]$ Mods - can this be made a sticky thread?

(Eden09, thread \#48, post 1)

In this thread, Eden09 (E), a female user with a journalistic streak, uses various ways to address ("Hi,") and involve her audience ("Feel free to ask any more questions $\left.:-)^{\prime}\right)$ - among them moderators in particular ("Mods - can this [...]"), at whom she directs a request. In so doing, she makes it perfectly clear that this thread does aim at interaction and that she is willing to be an active part of it. Accordingly, nine of the 21 following responses are produced by the thread starter herself, while all the other posts are crafted by five other users, all of whom are quite experienced TSR members. 
As could have been expected, their responses to the opening post mainly include thanking and praising the author for making the effort of compiling such a "Q\&A thread". Above that, some of them go into details about their professional aspirations or their doubts and worries:

Good thread $\oplus[\mathrm{W}[\mathrm{I}$ I was going to write the same sort of thing, because there are always SO many threads asking questions (especially that dreaded 'isn't a journalism undergrad degree useless?') and I have a list of questions that I was going to answer but I was too lazy to actually do it $(9)$

Good thread. I'm going to do a journalism degree at LJMU, hopefully. [...]

Others outspokenly disagree with some of the points made in the opening post:

NCTJ accrediation isn't vital, Cardiff and City, arguably the two best journalism schools in the UK don't offer it at undergraduate. There is a growing feeling that accredited undergraduate degrees are a waste of time and money - they don't teach you anymore then a NCTJ short course. [...] (p4)

It's funny that the newspapers are being mentioned... An astonishingly outdated medium that does not have much future to be honest - and yes, I know that some newspapers have been here for 100 years plus, but truth be said, the sale of newspapers is constantly declining. [...]

Based on these two types of responses, the thread starter $E$ embarks on several dialogic exchanges with her interlocutors: On the one hand, she wishes them luck, advises and encourages them, and generally shows interest in personal details disclosed by her fellow posters. On the other hand, controversial points are discussed vigorously but nevertheless factually. For the sake of illustration, two dialogic strings are displayed in the following:

\begin{tabular}{|l|l|l|l|}
\hline C: & p2 & $\begin{array}{l}\text { That was well written and informative, thanks }:-[\backslash] \text { I'm } \\
\text { hoping to get on to a course and do journalism next year... }\end{array}$ & im. + \\
\hline E: & p3 & $\begin{array}{l}\text { Good luck! Do you know where abouts yet? What alevels are } \\
\text { you doing? }\end{array}$ & im. + \\
\hline$C:$ & p8 & $\begin{array}{l}\text { I haven't } 9 \text { Ive got a NVQ in childcare, which i passed with } \\
\text { a distinction, and i am currently finishing 3 OU courses in } \\
\text { law, psychology and child psychology. I'm hoping that'll be } \\
\text { enough, or but I'm very scared that it won't. I've been pub- } \\
\text { lished a few times, though, so hopefully that'll help! [I] I’ve } \\
\text { applied for Chester and Birmingham City. Nervous! [I] Any } \\
\text { tips if i do get accepted? }\end{array}$ & im \\
\hline
\end{tabular}




\begin{tabular}{|l|l|l|l|}
\hline E: & p10 & $\begin{array}{l}\text { Law will be very useful and getting published is a big } \\
\text { achievement as well so you'll stand a fighting chance. }[\backslash] \\
\text { As for tips, do lots of work experience whilst you're on the } \\
\text { course, at the local paper, local radio station and you'll get } \\
\text { your name out there, build up some industry contacts. Also } \\
\text { do lots for the student paper and radio station too, they're } \\
\text { always good to bulk up the CV. Also start a blog and put a } \\
\text { link to it at the bottom of your emails etc. sometimes people } \\
\text { like to have samples of work they can read rather than you } \\
\text { just telling them how good you are in a CV } \because \\
\text { of finding contacts and interviewees for stories, don't tell } \\
\text { them you're a student, tell them you're a freelance journalist, } \\
\text { most people don't want to help students } 9\end{array}$ & im.
\end{tabular} \mid

Despite the fact that the next two interlocutors disagree, their exchange is not only characterized by long, well-wrought arguments but also by its factual style. As indicated by the underlined passages, users in this part of the thread take the high road in finding ways to exchange arguments and stay on-topic without making leeway into inappropriate, flaming behavior:

\begin{tabular}{|c|c|c|c|}
\hline$D:$ & p15 & $\begin{array}{l}\text { It's funny that the newspapers are being mentioned... An } \\
\text { astonishingly outdated medium that does not have much } \\
\text { future to be honest - and yes, I know that some newspapers } \\
\text { have been here for } 100 \text { years plus, but truth be said, the sale } \\
\text { of newspapers is constantly declining. [...] Sorry to say that, } \\
\text { but it's good to be aware of the fact that newspaper journal- } \\
\text { ism is not as an excellent of a career as it has been } 10-20 \\
\text { years ago, and probably never will be again } \bigcirc \\
\text { there are still gonna be newspapers in circulation, probably } \\
\text { for the next dozen years or so, but methinks they don't have } \\
\text { much future anyway } \because\end{array}$ & im. $\varnothing$ \\
\hline$E:$ & p16 & $\begin{array}{l}\text { Wow, you have some very strong views. } \\
\text { newspapers are the most traditional form of news, but not } \\
\text { really outdated. There are some people who feel that news- } \\
\text { papers are the only true form of media left as broadcast news } \\
\text { isn't allowed to be subjective, print is, and for the press to } \\
\text { fulfil their role as scrutinising the executive (fourth estate if } \\
\text { you want to google it) then it has to be subjective. }\end{array}$ & im. $\varnothing$ \\
\hline
\end{tabular}




\begin{tabular}{|c|c|c|c|}
\hline$D:$ & p17 & $\begin{array}{l}\text { IMO print is as much "subjective" as broadcast news and } \\
\text { vice versa. }[\backslash] \text { On the other hands - I always thought that it's } \\
\text { objectivity that matters and should be encouraged (however } \\
\text { hard to achieve as the final outcome), not the subjectivity, i.e. } \\
\text { "bias" (of whatever sort). } \\
\text { or less the same? (do they not mean more } \\
\text { speaker so lol } \stackrel{\text { correct me if I'm wrong, I'm not a native }}{-}\end{array}$ & $\operatorname{im} . \varnothing$ \\
\hline$E:$ & $\mathrm{p} 18$ & $\begin{array}{l}\text { Not really, most newspapers lean towards one end of the } \\
\text { political spectrum, right or left, and a few hover in the mid- } \\
\text { dle. This is what I meant by them having subjectivity, their } \\
\text { aim is to attract a particular audience and so they would } \\
\text { bring up the issues that are of concern to that audience. Does } \\
\text { that make sense? [ } \backslash] \text { Edit: just wanted to add that by law the } \\
\text { broadcast media isn't allowed to be subjective because they } \\
\text { are viewed by such a mass audience.. I'm not sure what the } \\
\text { laws are, I just know they're a lot tighter about what they are } \\
\text { allowed to broadcast. [ } \backslash] \text { And yeah, usually objectivity is [...] }\end{array}$ & im. $\varnothing$ \\
\hline$D:$ & p19 & $\begin{array}{l}\text { That's pretty interesting - where I'm from (Poland) the } \\
\text { broadcast media are pretty much subjective [...] Good to } \\
\text { know that, had no idea that there actually is law here that } \\
\text { explicitly forbids public broadcasters to steer off-centre }\end{array}$ & im. $\varnothing$ \\
\hline$E:$ & p20 & $\begin{array}{l}\text { Yea I can imagine it being very different in other countries, } \\
\text { you only have to watch FOX news to realise, I didn't believe } \\
\text { it was actual news the first time I saw it [...] Thinking about } \\
\text { it, I'm not sure if it's just the BBC who're obligated to remain } \\
\text { objective or all broadcasters... anyone else have any idea? (I } \\
\text { should probably know this being a journalist lol @g) }\end{array}$ & im. $\varnothing$ \\
\hline
\end{tabular}

The harmonious nature of this thread, which is pretty uneventful in terms of interpersonal relations, finds also expression in figures (see Table 34).

The other three threads of this category resemble thread \#48 to a great extent, which is why they will only be discussed very briefly. Again, they involve experienced thread starter in offering helpful information to his interlocutors, viz. helpful geography websites (\#38), the pointer to watch a meteor shower (\#5) and hints on how to study effectively (\#36). 
Table 34. Relational work in thread \#48

\begin{tabular}{|c|c|c|c|c|c|c|}
\hline \multirow{2}{*}{\multicolumn{2}{|c|}{$\begin{array}{l}\text { Type of post } \\
\text { and evaluation }\end{array}$}} & \multicolumn{3}{|c|}{ Functional $\rightarrow$ appropriate } & \multicolumn{2}{|c|}{ Dysfunctional $\rightarrow$ inappropriate } \\
\hline & & marked + & marked $+\mathrm{m}$ & unmarked & marked - & marked - m \\
\hline \multirow[t]{2}{*}{ A: 16} & ex. & 0 & 0 & & 0 & 0 \\
\hline & im. & 3 & 0 & 13 & 0 & 0 \\
\hline$B_{t}: 6$ & im. & 1 & 0 & 5 & 0 & 0 \\
\hline \multirow[t]{2}{*}{$\sum: 22$} & & 4 & 0 & 18 & 0 & 0 \\
\hline & & \multicolumn{3}{|c|}{22} & \multicolumn{2}{|c|}{0} \\
\hline
\end{tabular}

\section{Guide to How to Revise Effectively?}

How to Revise Effectively?

[ $I]$ This may be a little late for some people, but I was inspired to make this having seen so many people worried, depressed and generally down over exams, so here is a guide I use: [ $I]$ [detailed and structured description of a method to study] [ I] If you start your revision early, plan your revision carefully and follow your plan with those past papers, your chances of success are greatly enhanced. [ $\backslash]$ Good luck to all sitting exams now and in future!

(Lord Hysteria, thread \#36, post 1 )

This originally colorful entry was authored by Lord Hysteria (L), one of TSR many PS Helpers. It is very well structured and reveals detailed information, starts with an explanation on why he considers this thread necessary and closes with good luck wishes to those sitting exams. In doing so, he more or less directly addresses those to which this thread may come in handy. Towards the end, the intensity of his advice is emphasized by the change of color (to red) and style (italics). The cheerful smiley in terminal position serves as a closing and may have been used to stress the optimistic tone.

Interestingly, the first one to "reply" to the initial post is the thread starter himself, supplementing his guide with an explicit invitation to his fellow-users among them especially those who have been successful in the past - to discuss and share study methods and habits:

Now that I have put my section up, just thought it would be great to start an actual thread on studying habits. People who have seen great results please respond and let us know how you study and what was effective for you. So others can take some pointers. 
Accordingly, the first "real" response composed by one of his 30 partly inexperienced interlocutors can only be found in the third post. Since L's speech act is divided over two posts, with $\mathrm{p} 1$ containing the propositional and illocutionary act and $\mathrm{p} 2$ the perlocutionary act, thus expressing the thread starter's expectations for the progress of the thread, I will treat the two posts as one connected entry.

In accepting L's proposal for the continuation of the thread, his fellow-members embark on describing and evaluating methods, thereby sharing pointers partly via links - on how to study successfully. Above that, additional topic-related questions are asked and users, above all the thread starter, are thanked and praised for contributing great ideas. Dialogic structures, let alone polylogic structures, were, however, only far and few between. Here is a sample of users' responses:

What a good good boy. That's a very helpful guide.

[...] thanks for this Lord. you get my rep for today

thanx for the ideas guys. ive found that the whiteboard mind mapping is really workin 4 me $\oplus$

Can I add one? [ $\backslash$ ] Actually study in free periods. Requires willpower and immense self control but will be worth it.

this was really helpful! thanks alot! $<3$

The thread starter is a rather passive participant - with 7 out of 41 responses penned down by $L$ - who joins in the conversation only occasionally, mostly by thanking and accepting thanks by others but also by joking with them and giving additional advice:

I am a good boy! ;yes;

(O) Thankies [I] OK - No more spamming ...

no probs mate $\oplus$

Although basically characterized by a friendly atmosphere, even this thread has at least one passage in store in which the thread starter faces an appalled TSR user who accuses him of plagiarism:

\begin{tabular}{|l|l|l|l|}
\hline A: & p19 & $\begin{array}{l}\text { OMG - The guide has all been copied - I was just reading } \\
\text { my Letts KS3 Maths book and 90\% of it is all from the book. }\end{array}$ & im. $\varnothing$ \\
\hline$L:$ & p20 & $\begin{array}{l}\text { so what? [ } \backslash] \text { Not everyone can access Letts, and I so happen } \\
\text { to think it is worth it. }\end{array}$ & im. $\varnothing$ \\
\hline
\end{tabular}




\begin{tabular}{|c|c|c|c|}
\hline A: & $\mathrm{p} 21$ & $\begin{array}{l}\text { wtf are you on about? you obviously copied it out of a letts } \\
\text { book and claimed it as your own. That's plagarism and can } \\
\text { get you into some serious trouble..... }\end{array}$ & ex. - \\
\hline$L:$ & p22 & $\begin{array}{l}\text { where? } \oplus[\backslash] \text { Unlike coursework, where you have to sign } \\
\text { a document saying it is yours, I never at any point said it is } \\
\text { mine. I think you have been doing too much coursework. } \\
\end{array}$ & im. $+\mathrm{m}$ \\
\hline
\end{tabular}

In keeping his cool, $L$ manages to take the wind out of $A$ 's sails. Instead of letting the conversation degenerate into an unpleasant exchange of accusations, $L$ calmly brings forward arguments in his favor and ends with a nice example of mock impoliteness - maybe to conciliate his interlocutor. Whether this is successful is uncertain since there is not further response by $A$. And another thing is uncertain: Although using quite expressive acronyms ("wtf", "omg") to articulate his emotions, we cannot even know if $A$ was not mocking $L$ all along.

What is interesting about this thread is the fact that the pair of opening posts are not judged unanimously. Instead, votes are divided between two types of appropriate behavior: While 15 users saw p1 as unmarked (“im. Ø”), 8 evaluated it explicitly positive and thus as polite ("ex. +"). The initial post thus needs to be noted down as an exception.

\section{Jolly useful Geography websites}

Edited to update useful sites. I will add others in as people put them on this thread [This is a copy of the one in the Secondary Geography thread!] [1] Here's an updated list of all the others in this thread [ $\backslash$ ] [list comprising 37 links to websites, each furnished with a comment about the content]

(Geogger, thread \#38, post 1)

Geogger $(G)$, another experienced PS Helper to initiate a thread, is an expert on traveling and geography, which is why she is willing to share her knowledge with others. In contrast to the preceding thread, $G$ solely focuses on technical and content related details, thus leaving out any interpersonal gestures such as addressing interlocutors, inviting them to share their knowledge or to contribute to a lively discussion. In this way, it is totally left to the 48 interlocutors to react appropriately to this thread start.

Again, the most obvious reaction to this kind of thread opening is thanking its author. Far more characteristic for this thread are, however, discussions about the usefulness of this link or another. Above that, this thread also prompted users to contribute their own favorite links, to ask for other project-specific links and to offer their help voluntarily. A "best of"-list of all these possible reactions could look like this: 
Godammit!! I wish id been on here during my A levels. Lol שึ]

Omg, this site is majic thankyou sooooo much!

woo! thanx geogger! $\because$ ) so efficient $\oplus$ hehe

oooo, thats a good one! $(9)$

Looking for something for your presentation? [ । Then [link] may be helpful. The most important statistics of a country in one image. Copy \& Paste it into your work.

After the initial post, the thread starter almost vanishes completely, never to be seen again and resurfacing only one more time in $\mathrm{p} 85$. Traces of her participation can only be found in the form of quotes, which are embedded in her fellowmembers' posts - with her original contribution however missing and thus not counting for this study.

\section{Meteor Shower :)}

Hi guys,

Just to let you all know next tuesday (12th of August) there will be a meteor shower (Perseids), the best of 2008 the experts say.

Those of you in the countryside will see a lot, and I saw them last year, one of the best experiences in my life. Unfortunatly I live in London so light pollution resricts me, but fear not I'll be making an effort to go to the countryside - it really is worth it

Would be good for you all to see it, especially as its two days before AS/A-level results, might calm your nerves :P

(Arsenal4life, thread \#5, post 1)

Last but not least, thread \#5 was composed by Arsenal4life, who decided to pass on some information he considers to be useful for his stressed-out fellow TSRmates, which he directly addresses more than once ("Hi guys", "to let you all know", "those of you", "good for all of you", "might calm your nerves"). This considerate and selfless deed triggered 73 responses, three of which have been authored by the thread starter, again to offer additional information during rather short-lived dialogic exchanges. Slightly contradictory to his initial attitude of friendliness, two out of his three interjections are marked by mock-impoliteness, as he calls one of his interlocutors a "lucky $b^{* * * * *} \mathrm{~d}: \mathrm{P}$ " (p18), another one "smartass [...] (:)" (p28).

Similar to the preceding threads, users exchange information and experiences, some also express thanks to the thread starter. Allusions to meteor-related pop culture and literature is a special means of bonding in this particular thread - and obviously also of misunderstandings, as the following excerpt from a more complex polylogue illustrates: 


\begin{tabular}{|c|c|c|c|}
\hline$s:$ & p6 & $\begin{array}{l}\text { ooh sounds fun. Just remember: don't look at the green } \\
\text { meteors. }[\backslash] \text { (Sorry, a weird reference from a book no one } \\
\text { has probably really read) }\end{array}$ & $\operatorname{im} . \varnothing$ \\
\hline$p:$ & p7 & Will anyone be hurt/maimed? & im. $\varnothing$ \\
\hline$N:$ & p9 & $\begin{array}{l}\text { No don't worry, they burn harmlessly as they enter the } \\
\text { Earth's atmosphere } ;\end{array}$ & im. + \\
\hline$p:$ & p10 & Epic fail. 6 & im. $-\mathrm{r}$ \\
\hline$s:$ & $\begin{array}{l}\mathrm{p} 8 \\
(\rightarrow 7)\end{array}$ & $\begin{array}{l}\text { They'll go blind, and then be eaten by genetically engineered } \\
\text { walking, hunting man eating plants! [ } \backslash] \text { (I think you're talk- } \\
\text { ing to me? }\end{array}$ & im. $\varnothing$ \\
\hline J: & p14 & Triffids! 89 one of my fave books ever! $\Theta$ & im. Ø \\
\hline
\end{tabular}

Since $N$ obviously did not notice the allusion, he answers the question literally and to his best knowledge and belief. In so doing, he even shows consideration for the user he considers to be worried, $p$. Instead of uncovering the misunderstanding and/or showing gratefulness for the other's concern, $p$ answers with rudeness. In the continuation of this exchange, another Triffids-remark meets with so much approval and excitement that $s$ is willing to give $J$ another round of "reputation", thereby doing positive facework and strengthening their bond: "aahaha I'd rep you again but alas

In contrast to other categories and owed to the altruistic purpose of this category, the facework is more than ever based on thankfulness and sharing. Thread starters are praised for the kind contributions and sometimes even rewarded with "reputation". Users share their knowledge (e.g. by means of links), experiences, feelings and worries, encourage and wish each other luck. Above that, they bond over shared topics and interests and try to avoid misunderstandings as soon as possible. Naturally, some aspects are discussed ardently, others provocatively, leading in some rare cases to dysfunctional communication. This is also mirrored in the final account, which gives a valid picture of the entire category (see Table 35).

As could have been predicted by the relatively low IQs and the altruistic intentions of the four thread starters - among them Lord Hysteria (\#36) and Geogger (\#38), both of whom have been labeled "do-gooders" before -, this category is yet another epitome of appropriate behavior with 213 to 2 cases. The introductory posts of threads \#5 and \#36 elicited divided evaluations insofar as some users considered them unmarked, but nevertheless appropriate, while others indicated a decidedly positive evaluation through their responses. 
Table 35. Relational work in the sixth category (Offering advice/information)

\begin{tabular}{|c|c|c|c|c|c|c|}
\hline \multirow{2}{*}{\multicolumn{2}{|c|}{$\begin{array}{l}\text { Type of post } \\
\text { and evaluation }\end{array}$}} & \multicolumn{3}{|c|}{ Functional $\rightarrow$ appropriate } & \multicolumn{2}{|c|}{ Dysfunctional $\rightarrow$ inappropriate } \\
\hline & & marked + & marked + m & unmarked & marked - & marked - m \\
\hline \multirow[t]{2}{*}{ A: $72^{*}$} & ex. & 10 & 0 & & 1 & 0 \\
\hline & im. & 6 & 1 & 54 & 0 & 0 \\
\hline $\mathrm{B}_{\mathrm{t}}: 143$ & im. & 21 & 3 & 118 & $1(1 \mathrm{r})$ & 0 \\
\hline \multirow{2}{*}{\multicolumn{2}{|c|}{$\sum: 215(+8 \mathrm{C})$}} & 37 & 4 & 172 & $2(1 \mathrm{r})$ & 0 \\
\hline & & \multicolumn{3}{|c|}{213} & \multicolumn{2}{|c|}{$2(1 \mathrm{r})$} \\
\hline \multicolumn{7}{|c|}{${ }^{*}+2$ exceptions } \\
\hline
\end{tabular}

\subsection{Summary: Findings for the management of interpersonal relations}

In focusing on users' reactions to the propositional content and/or the phrasing of thread starts and subsequent dialogic and polylogic structures, this chapter examined the management of interpersonal relations among interlocutors in all 50 threads of this message board corpus. In doing so, two strands of investigation were pursued for all six purpose-driven categories: Qualitatively, we found out about participants' facework strategies and illustrated their linguistic implementation with the help of selected examples. The quantitative analysis provided an overview of the types of relational work, which were also exemplified by means of message board excerpts. The only thing left to do is to bring together the results of the six categories in order to make a point about facework and relational work witnessed in the entire corpus.

With regard to facework, participants resorted to various strategies to either support or subvert others' needs for association and dissociation - thereby naturally affecting their own face needs accordingly. In order not to list these strategies one by one again, the following overview bears witness to the attempt to condense them into somewhat broader, yet certainly variable and anything but exhaustive categories:

\section{A user's $\left(U_{1}\right)$ need for association}

... finds expression in $U_{1}$ 's

- greeting and thanking others (also in advance);

- signaling interest in a topic and/or in joining a topic related society;

- sharing of personal information, experiences, emotions and opinions; 
- offering or asking for advice, sympathy and friendship;

- joking with others;

- attempting to keep a conversation going;

- dropping lines (laying the groundwork for face-negotiations);

- praising interlocutors for their contributions and/or efforts made, also by "repping" others positively;

- $\quad$ apologizing for lengths of entries, topic drifts, outbursts of emotions etc.;

- making the tentative nature of opinions explicit;

- negotiating meaning ( $v i z$. reference, phrasing etc.) to clarify content and in order to avoid misunderstandings and friction;

- discussing the code of conduct.

$\ldots$ is supported by his fellow-users $\left(\mathrm{U}_{2-\mathrm{n}}\right)$ by means of their

- welcoming $\mathrm{U}_{1}$ (in the group) / offering in-group membership;

- offering help, advice, warnings;

- sharing personal information, experiences, emotions and opinions;

- picking up and elucidating on topics introduced by $\mathrm{U}_{1}$, thus creating common ground and topical cohesion;

- discovering commonalities, teaming up, bonding;

- sympathizing with $\mathrm{U}_{1}$, cheering him up, encouraging him, comforting him, wishing him luck;

- approving of $\mathrm{U}_{1}$ 's humorous remarks;

- agreeing with lines of argumentation/opinions;

- defending $U_{1}$ against others;

- praising $\mathrm{U}_{1}$ for his contribution(s) and/or efforts made, also by "repping" him positively;

- confirming face claims;

- reciprocating relational work, for example by completing adjacency pairs (i.e. greeting back, thanking him etc.).

$\ldots$ is subverted by his fellow-users $\left(U_{2-n}\right)$ by means of their

- ignoring $\mathrm{U}_{1}$;

- parading disinterest;

- excluding $\mathrm{U}_{1}$ of the in-group;

- patronizing, blaming, mocking, insulting $\mathrm{U}_{1}$;

- criticizing the content of $U_{1}$ 's post, attitude, opinions, or the choice of topic as such;

- doubting the truthfulness of $\mathrm{U}_{1}$ 's story;

- not answering in a topic related manner, e.g. by means of joking or ironic comments; 
- attributing unfavorable face claims to $\mathrm{U}_{1}$;

- "repping” $U_{1}$ negatively.

\section{A user's $\left(U_{1}\right)$ need for dissociation}

... finds expression in $\mathrm{U}_{1}$ 's

- staying vague when disclosing personal information (incl. avoiding dropping lines);

- refusing to join a society, ignoring offers of friendship;

- stating disinterest in the topic;

- giving ironic answers;

- criticizing the (phrasing of the) topic;

- disagreeing with the views of others, contradicting them;

- dropping out of the conversation;

- evaluating others' responses negatively;

- patronizing, blaming, mocking, insulting others;

- violating the code of conduct (without apologizing).

... is supported by his fellow-users $\left(\mathrm{U}_{2-\mathrm{n}}\right)$ by means of their

- accepting diverging opinions without picking a fight over it;

- ganging up with $\mathrm{U}_{1}$ against prevailing opinion(s) (thus giving $\mathrm{U}_{1}$ a sense of association by agreeing with them, see above);

- not pressuring $\mathrm{U}_{1}$ into disclosing further information.

$\ldots$ is subverted by his fellow-users $\left(\mathrm{U}_{2-\mathrm{n}}\right)$ by means of their

- not accepting diverging opinions; trying to convince $U_{1}$ of opposing views, if necessary by force (i.e. insults, flames etc.);

- relativizing or downplaying a particular problem;

- pressing for more information;

- calling $\mathrm{U}_{1}$ to order, for example to respect the code of conduct.

As stated before, these kinds of lists can never be exhaustive, nor is it possible to avoid overlaps in certain strategies, as both sides of the coin, supportive and subversive facework, are closely associated with each other. Obviously, there are far more strategies for positive facework than for its negative counterpart - a fact which perfectly mirrors the general purpose and nature of message boards as a place for gathering and association of like-minded people in the broadest sense. Accordingly, the human need for dissociation plays a subordinate role in this type of communication.

Interestingly, behavior did not only vary with regard to the orientation of facework but in some cases also according to the status of the participants within TSR. Especially in the first category (Getting to know others), a difference between 
savvy regulars on the one hand and inexperienced newbies on the other hand was noticeable. While newbies did not seem to venture too much in their introductory posts - content-wise and interpersonally - and often stuck to the pattern of the preceding entry rather closely, experienced members acted more self-confident, thus composing decidedly more idiosyncratic contents. Then again, moderators and other TSR "officials" remained on the sidelines most of the time, appearing more often than not as interested fellow-interlocutors rather than interfering supervisors.

In this chapter, attention was also paid to the behavior of some noticeable thread starters throughout their respective threads, referring to the five "do-gooders" and seven "trouble makers" introduced in Section 7.3.2. Due to the lack of active participation, it turned out that assumed reputations could not always be falsified, like that of Geritak (\#15), Geogger (\#38) and Sahds (\#42). Others, like the "do-gooders" Lord Hysteria (\#36) and MelonFace (\#46), proved to be worthy of the reputation previously derived from the information contained in various templates, as they excelled by their consistent appropriate, at times even positively marked behavior. The investigation of the "trouble makers" on the other hand, yielded more heterogeneous results. While some of them, such as Adonis (\#13), Neo Con (\#17), Don_Scott (\#27) and Barz (\#29), behaved contrary to their negative reputation by making perfectly acceptable, at times even considerate contributions, others conducted themselves in more predicable ways: ShiVji (\#8) and $9 \mathrm{MmBulletz}$ (\#34), although contributing seldom and not stirring up any trouble, made themselves conspicuous by their outspoken and impatient streak. Last but not least, Mr_Mad's (\#28) behavior clearly indicated that he was asking for trouble. Just by looking at the choice of his topic and the explicit wording of his heading (Women Make Crappy Musicians), let alone the challenging, even condescending tone of his contributions, it was soon obvious that he absolutely lived up to his reputation.

Reconsidering the numerous examples provided in the course of this chapter, we can also make some generalizations about the formal side of these strategies. Although contributions vary considerably in terms of length and elaborateness, one cannot help but notice that compensatory mechanisms are used fairly consistently to make up for the lack of para- and nonverbal clues in this type of computer-mediated environment. As predicted in Section 2.6.2, users tended to emphasize the content of their statement and their underlying emotional stances towards their interlocutors by means of deliberate color, font and size changes and with the help of emulated speech, capitals and most of all smileys. Especially the latter ones, be it in animated form or furnished with signposts, came in handy to reproduce non-verbal behavior iconically, among others hugging, high-fiving, or flipping somebody the bird, or to convey opinions quite outspokenly. Above that, 
Table 36. Availability versus trustworthiness of evaluations in type A and $B_{t}$ posts

\begin{tabular}{llll}
\hline & $\begin{array}{l}\text { type A, ex. } \\
\text { (explicit evaluation) }\end{array}$ & $\begin{array}{l}\text { type A, im. } \\
\text { (implicit evaluation) }\end{array}$ & $\begin{array}{l}\text { type } \mathrm{B}_{\mathrm{t}} \text {, im. } \\
\text { (implicit evaluation) }\end{array}$ \\
\hline availability & low & medium & high \\
trustworthiness & high & medium & low \\
\hline
\end{tabular}

the multimodal potential has also been made use of more than once by users who decided against a verbal response in favor of embedded pictures, videos and links to other websites. As a consequence, the negotiation of interpersonal relations does not - as feared - fall by the wayside in a form of communication that tends to favor an economic mode of speaking. Instead, the negotiation process can, but does not necessarily have to, take on a more economic form. For that reason, economic ways of expression and interpersonal negotiations are not mutually exclusive categories.

As already indicated by the mean interaction quotient (IQ) of $37 \%$ for all threads, the degree of dialogicity, let alone polylogicity, fell short of our expectations. For that reason and to draw a valid picture of relational work in this kind of message board, we could not limit ourselves to dialogic or polylogic structures, but included all type $A$ and type $B_{t}$ posts, independent of their position within the interaction structure of the thread. With regard to the availability but also the trustworthiness of evaluations of relational work to be found in posts, Table 36 presents itself. Obviously, there are a lot of type $B_{t}$ posts (see also Table 37), which are - due to their very nature (no subsequent post and hence no evaluation) - the least trustworthy, and a smaller amount of type A posts with a medium to high degree of trustworthiness. Unfortunately, though, even type A posts with one, sometimes even two or more subsequent posts are not always explicitly assessed, as not every follow-up contribution contains evaluative statements about its predecessor. For these reasons, the majority of posts are not explicitly evaluated at all, which is why the participant observer perspective gains momentum and cannot be neglected. For the sake of transparency, the least we can do, however, is to separate these two perspectives, interlocutors' explicit and participant observers' implicit evaluations. Accordingly, the final account for all 50 threads is presented in Table 37.

The final results as portrayed in this table could hardly be clearer and go hand in hand with the previously established fact that there is a predominance of users' need for association over their need for dissociation when getting together in message boards: In a ratio of approximately 12:1 posts, participants' behavior in this corpus is primarily appropriate $(92.3 \%)$ and seldom characterized by inappropriateness (7.7\%). The numerical superiority of the former category obviously stems from the fact that it comprehends a huge amount of unmarked, yet 
Table 37. Relational work in all 50 threads

\begin{tabular}{|c|c|c|c|c|c|}
\hline \multirow{2}{*}{$\begin{array}{l}\text { Type of post } \\
\text { and evaluation }\end{array}$} & \multicolumn{3}{|c|}{ Functional $\rightarrow$ appropriate } & \multicolumn{2}{|c|}{ Dysfunctional $\rightarrow$ inappropriate } \\
\hline & $\begin{array}{l}\text { marked + } \\
\text { "polite" }\end{array}$ & $\begin{array}{c}\text { marked + m } \\
\text { "mock-impolite" }\end{array}$ & $\begin{array}{c}\text { unmarked } \\
\varnothing\end{array}$ & $\begin{array}{l}\text { marked - } \\
\text { "impolite" } \\
\text { ("rude" = r) }\end{array}$ & $\begin{array}{l}\text { marked - m } \\
\text { "mock-polite" }\end{array}$ \\
\hline \multirow[t]{2}{*}{ A: $1232^{*} \mathrm{e}$} & 73 & 1 & & $55(32 r)$ & 6 \\
\hline & 35 & 2 & 1017 & $39(24 r)$ & 4 \\
\hline $\mathrm{B}_{\mathrm{t}}: 1984 \mathrm{im}$. & 94 & 10 & 1736 & $130(81 \mathrm{r})$ & 14 \\
\hline \multirow[t]{2}{*}{$\sum: 3216(36 \mathrm{C})$} & 202 & 13 & 2753 & $224(137 r)$ & 24 \\
\hline & \multicolumn{3}{|c|}{2968} & \multicolumn{2}{|c|}{$248(137 r)$} \\
\hline${ }^{\star}+16$ exceptions & $\begin{array}{l}\# 4 \mathrm{p} 17: \mathrm{e} \\
\# 5 \mathrm{p} 1: \mathrm{in} \\
\# 9 \mathrm{p} 28: \mathrm{i} \\
\# 10 \mathrm{p} 110 \\
\# 11 \mathrm{p} 1: \mathrm{i} \\
\# 11 \mathrm{p} 7: \mathrm{i} \\
\# 13 \mathrm{p} 30: \\
\# 13 \mathrm{p} 38:\end{array}$ & $\begin{array}{l}\text { x. - r., ex. - m } \\
\varnothing, \text { ex. + } \\
\text { m. } \varnothing \text {, im. - r } \\
\text { im. } \varnothing, \text { im. + (typ } \\
\text { m. }, \text { im. - r } \\
\text { m. } \varnothing \text {, im. - r } \\
\text { im. } \varnothing \text {, im. - r } \\
\text { im. +, ex. - r }\end{array}$ & $\left.B_{t}\right)$ & $\begin{array}{l}\# 15 \text { p13: im. } \varnothing \\
\# 19 \text { p53: im. } \varnothing \\
\# 28 p 1: \text { im. } \varnothing, \\
\# 36 p 1: \text { im. } \varnothing, \\
\# 41 \text { p1: im. } \varnothing, \\
\# 44 p 6: \text { im. } \varnothing, \\
\# 45 \text { p6: im. } \varnothing, \\
\# 45 p 12: \text { im. } \varnothing\end{array}$ & $\begin{array}{l}x .-r \\
x .+ \\
-r \\
+ \\
+-r \\
- \\
-\end{array}$ \\
\hline
\end{tabular}

appropriate behavior with 2753 posts, representing $92.8 \%$ of the entire category of functional communication (and still $85.6 \%$ of message board communication in its entirety). This behavioral type stands in marked contrast to its two co-constituents in the category of appropriate behavior, namely politeness with $6.8 \%$ and mock-impoliteness with only $0.4 \%$. Obviously, the use of impoliteness to mock interlocutors is extremely rare.

When looking only at the second half of Table 37, mock-politeness is also outnumbered by impoliteness/rudeness with $9.7 \%$ to $90.3 \%$. Remarkable for that category, though, is the proportion of impoliteness and rudeness: 137 of 224 negatively marked, impolite posts enclosed hints to their author's hurtful intention, which is why they have also been labeled rude. Percentage-wise, we are talking about $61.2 \%$ of negatively marked, impolite behavior and still $55.2 \%$ of all cases of dysfunctional, inappropriate behavior. This goes to show that once interlocutors make the (unconscious) decision to reply in an offensive manner, they do so quite straightforwardly and unambiguously, not veiling their spiteful intention.

As the analysis has also shown, the initial sum total of 3271 posts (see Table 15, Section 7.2) needs to be corrected slightly: Since the authors of threads $\# 29,34$ and 36 were also the driving forces behind the second post in each of the three threads, p2 did not count as an autonomous post but as the continuation of the first one. Accordingly, three posts need to be subtracted from the original 
Table 38. Incidences of relational work in the entire message board corpus

\begin{tabular}{|c|c|c|c|c|}
\hline \multicolumn{5}{|c|}{ Relational work } \\
\hline $\begin{array}{l}\text { negatively } \\
\text { marked }\end{array}$ & $\begin{array}{l}\text { (positively) } \\
\text { marked }\end{array}$ & unmarked & $\begin{array}{l}\text { positively } \\
\text { marked }\end{array}$ & $\begin{array}{l}\text { negatively } \\
\text { marked }\end{array}$ \\
\hline $\begin{array}{l}\text { impolite } \\
\text { (rude) }\end{array}$ & $\begin{array}{c}\text { mock-impolite } \\
\text { banter }\end{array}$ & non-polite & polite & $\begin{array}{l}\text { over-polite } \\
\text { mock-polite }\end{array}$ \\
\hline inappropriate & appropriate & appropriate & appropriate & inappropriate \\
\hline- & $+\mathrm{m}$ & $\varnothing$ & + & $-\mathrm{m}$ \\
\hline $\begin{array}{c}=224(137 r) \\
(7 \%)\end{array}$ & $\begin{array}{c}=13 \\
(0.4 \%)\end{array}$ & $\begin{array}{l}=2753 \\
(85.6 \%)\end{array}$ & $\begin{array}{l}=202 \\
(6.3 \%)\end{array}$ & $\begin{array}{c}=24 \\
(0.7 \%)\end{array}$ \\
\hline
\end{tabular}

sum total. Subtracting 36 type $\mathrm{C}$ posts and 16 exceptions, we are finally in the position to make a point about the incidences of relational work in the remaining 3216 posts of our message board corpus (see Table 38). The numerical range of relational work as found in the message board corpus can also be visualized with the help of a pie chart (see Figure 21).

Although not a part of the framework of relational work, we should still make a point about the occurrence of flames. As has been established before (see Section 5.7), not every impolite or rude utterance counts as a flame. When looking through the six categories once more and by reevaluating strings of negatively marked behavior, we encounter 26 flames in 14 threads (see Table 39). With the exception of the first (Getting to know others) and the sixth category (Offering advice/information), all other categories featured more than one flame in one or more threads, with the fourth category leading the way and the third category close behind. This result is not surprising, as it actually matches the six different purposes and interpersonal orientations of each category (see also Table 41). Lengths of flames varied: While most flames consisted of the minimal amount

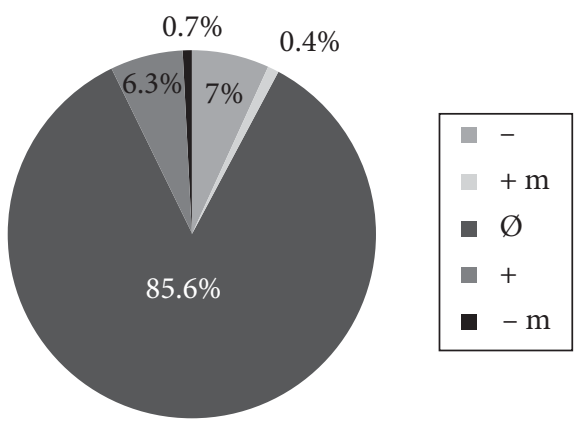

Figure 21. Percentages of relational work in the entire message board corpus 
Table 39. Incidences of flaming in all six categories

\begin{tabular}{ll}
\hline Categories & No. of flames \\
\hline 1. Getting to know others & 0 \\
2. Looking for like-minded others & 2 (in 1 thread) \\
3. Asking for advice & 9 (in 5 threads) \\
4. Asking for opinions & 13 (in 7 threads) \\
5. Blowing off steam & 2 (in 1 thread) \\
6. Offering advice/information & 0 \\
\hline$\sum$ & 26 (in 14 threads) \\
\hline
\end{tabular}

Table 40. Types of relational work used in flames

\begin{tabular}{lll}
\hline & Explicit evaluations & Implicit evaluations \\
\hline neg. marked & 16 & 15 \\
neg. marked rude & 22 & 17 \\
neg. marked mock / over polite & 7 & 3 \\
unmarked & - & 4 \\
\hline
\end{tabular}

of posts needed for a flame, two (17x), we could also find flames with three (3x), four (1x), five (1x), six (1x), seven (1x) and eight (1x) posts. The longest flame to be found in this corpus comprehended 11 posts. Accordingly, an average flame was 3.2 posts long. Flames exhibited the assortment of negatively marked (and unmarked) behavior (see Table 40).

These figures prove what has already been predicted in Section 5.7: Explicit user evaluations of behavior are particularly relevant in the context of flames, as this is the first time that they outnumber implicit participant observer evaluations with 45 and 35 cases respectively. In accordance with the nature of flaming, hurtful intentions are seldom veiled, which is why rudeness can be counted more often than impoliteness in both explicit and implicit perspectives. Every so often - four times to be more precise - flames were interspersed with unmarked entries from users who commented on the ongoing flame, sometimes in order to end it peacefully. In a nutshell, these figures attest that once users decide to start a fight with someone, they do it - not unlike a tempest - unvarnished but rarely persistently.

What still needs to be discussed shortly are the so-called exceptions, which have so far been bracketed out of the final results (see Table 37). Since these 16 entries could not be evaluated unanimously, they escaped the attribution to one of the above categories of relational work. As a matter of fact, we can distinguish two subtypes among these exceptional posts: those which received more than one evaluation (type A only) and those which contained more than one type of 
relational work (type A and B). While the former constitute the lion's share of the exceptions, the latter are very rare and can only be found in contributions, which answer to two interlocutors in two diverging strings of conversation by means of one and the same entry. Consequently, evaluations of these posts saw combinations of labels, mostly of unmarked and (negatively or positively) marked behavior. In the case of type A posts, these labels could also vary between implicit and explicit perspectives based on one or more subsequent posts referring to the one in question. Never have evaluations varied that much, however, as to produce a combination of positively and negatively marked behavior - a clue for the interpretative boundaries even of online contributions. Although evaluations of p38 in thread \#13 hinted to two types of relational work, "im. +" and "ex. - r", we have to keep in mind that the author used this particular post to react to the behavior of two separate interlocutors.

For the sake of completion, one should not forget to mention either that five of the 16 exceptions were found in thread initial position. Although the content and/or the phrasing of thread starts usually received "im. $\varnothing$ ", two of these five were also explicitly considered as polite (\#5 Meteor Shower:), \#36 Guide to How to Revise Effectively?), one explicitly as rude (\#28 Women Make Crappy Musicians) and the last two implicitly as rude (\#11 What does this girl mean by this? (convo included), \#41 So it seems everyone just wants to ruin there livers in university).

Speaking of initial posts, we are now in the position to make an educated guess as to why seven of the 50 thread starters changed their initial posts only shortly after posting their original draft (see Section 7.5). In fact, users in threads \#6 and 44 did not produce any inappropriate utterances, which means that - at least as a minimal effect - clarifications volunteered by thread starters in their modified second drafts did not cause any friction. A more positive evaluation could even hold that these modifications avoided friction and misunderstandings right at the outset. Since interlocutors did not thematize these particular parts of the introductory posts, there is no way of knowing for sure about their impact on the conversationalists. In the case of threads \#30, 37 and 39, we are still in the dark about what made the thread starter change his entry. Negatively marked behavior in these threads is in no way related to the respective thread starts. Reactions in the remaining two threads (\#4 and 46) are, however, a little more revealing: The thread starter of \#4 was attacked by two other users because of his (presumed) opinion on the proposed topic. For that reason, he saw a need for clarification about his real point of view, in order to avoid further negative reactions and misunderstandings. As interlocutors' behavior in the rest of the thread proves, this was the right decision. The situation is different in thread \#46: In conceding that his views may be completely wrong, the thread starter achieved the exact opposite 
Table 41. Percentages of relational work according to categories

\begin{tabular}{|c|c|c|c|c|c|}
\hline & \multicolumn{3}{|c|}{ Functional $\rightarrow$ appropriate } & \multicolumn{2}{|c|}{$\begin{array}{c}\text { Dysfunctional } \rightarrow \\
\text { inappropriate }\end{array}$} \\
\hline & $\begin{array}{l}\text { marked + } \\
\text { "polite" }\end{array}$ & $\begin{array}{c}\text { marked + m } \\
\text { "mock- } \\
\text { impolite" }\end{array}$ & $\begin{array}{c}\text { unmarked } \\
\varnothing\end{array}$ & $\begin{array}{l}\text { marked - } \\
\text { “impolite" } \\
\text { ("rude" = r) }\end{array}$ & $\begin{array}{c}\text { marked - m } \\
\text { "mock- } \\
\text { polite" }\end{array}$ \\
\hline \multirow{2}{*}{$\begin{array}{l}\text { 1. Getting to know } \\
\text { others }\end{array}$} & 33.3 & 1.7 & 65.0 & 0 & 0 \\
\hline & \multicolumn{3}{|c|}{100} & \multicolumn{2}{|c|}{0} \\
\hline \multirow{2}{*}{$\begin{array}{l}\text { 2. Looking for like- } \\
\text { minded others }\end{array}$} & 10.8 & 1.7 & 82.1 & 5.0 & 0.4 \\
\hline & \multicolumn{3}{|c|}{94.6} & \multicolumn{2}{|c|}{5.4} \\
\hline \multirow[t]{2}{*}{ 3. Asking for advice } & 6.6 & 0.4 & 79.3 & 12.7 & 1.0 \\
\hline & \multicolumn{3}{|c|}{86.3} & \multicolumn{2}{|c|}{13.7} \\
\hline \multirow{2}{*}{$\begin{array}{l}\text { 4. Asking for } \\
\text { opinions }\end{array}$} & 3.0 & 0 & 90.8 & 5.5 & 0.7 \\
\hline & \multicolumn{3}{|c|}{93.8} & \multicolumn{2}{|c|}{6.2} \\
\hline \multirow[t]{2}{*}{ 5. Blowing off steam } & 9.2 & 0.6 & 78.6 & 9.8 & 1.8 \\
\hline & \multicolumn{3}{|c|}{88.4} & \multicolumn{2}{|c|}{11.6} \\
\hline \multirow{2}{*}{$\begin{array}{l}\text { 6. Offering advicel } \\
\text { information }\end{array}$} & 17.2 & 1.9 & 80.0 & 0.9 & 0 \\
\hline & \multicolumn{3}{|c|}{99.1} & \multicolumn{2}{|c|}{0.9} \\
\hline
\end{tabular}

effect. As the reaction "Don't be silly. This isn't about right or wrong." (p37) shows, his attempt to avoid friction obviously backfired.

In order not to judge all categories by the same yardstick, the last overview presents the final results once more in a more detailed manner. Note, that in Table 41 , each of the six categories represents $100 \%$. When looking at the high percentages for functional, appropriate behavior in general, ranging from a minimal of $86.3 \%$ to a maximum of $100 \%$, it becomes obvious that all of the categories sported mostly smooth interaction. As a result, the percentages for inappropriate behavior are rather low with $0 \%$ to $13.7 \%$ only. According to this overview, the first category, Getting to know others, was characterized by harmonious exchanges only, with the highest amount of politeness (33.3\%) of all six categories. This result is almost self-explanatory and quite predictable with regard to the purpose of this category, namely associating with TSR strangers. In the course of introducing oneself to a broader audience, controversial topics are least likely to come up. Still, belligerent exchanges can never be ruled out completely for any of these categories or any particular topic for that matter. It is, however, the third group, Asking for advice, which contained most friction with the lowest percentage of 
Table 42. Ranking of the six categories according to the appropriateness of behavior

\begin{tabular}{lccc}
\hline & $\begin{array}{l}\text { Appropriate } \\
\text { behavior }\end{array}$ & $\begin{array}{l}\text { Inappropriate } \\
\text { behavior }\end{array}$ & Average IQ \\
\hline Cat. 1: Getting to know others & $100.0 \%$ & $0.0 \%$ & $66 \%$ \\
Cat. 6: Offering advice/information & $99.1 \%$ & $0.9 \%$ & $45 \%$ \\
Cat. 2: Looking for like-minded others & $94.6 \%$ & $5.4 \%$ & $48 \%$ \\
Cat. 4: Asking for opinions & $93.8 \%$ & $6.2 \%$ & $38 \%$ \\
Cat. 5: Blowing off steam & $88.4 \%$ & $11.6 \%$ & $43 \%$ \\
Cat. 3: Asking for advice & $86.3 \%$ & $13.7 \%$ & $23 \%$ \\
\hline
\end{tabular}

appropriate behavior (86.3\%) and the highest (13.7\%) for impolite or even rude behavior. Outpacing even categories such as 4 (Asking for opinions) and 5 (Blowing off steam), which could have been expected to cause more dysfunctional communication, the third category saw quite dubious thread starters, with Anonymous User \#1 leading the way. In some cases, these users and/or the topics introduced by them were not taken seriously or were straightforwardly considered fake, thus eliciting fierce quarrellings more than once.

To sum it all up, let us classify the six categories according to the appropriateness (and inappropriateness) of their participants' behavior, incorporating also the average interaction quotient (IQ) for each category. As can be learnt from Table 42, categories 1 and 6 form the leading group in terms of functional behavior, while categories 2 and 4 constitute the midfield with categories 5 and 3 coming last. What is especially interesting about this overview is the correlation of the threads' average interaction quotients and their participants' conduct. Although the average values must not be overestimated - after all IQs varied considerably within the six categories - it is most striking that the category with the highest average IQ (66\%) is also the one highest in terms of appropriate behavior, while the category with the lowest average IQ (23\%) ranks also lowest with regard to interpersonal conduct. This suggests a positive correlation between the degree of interaction and the functionality of the exchange, which is, however, proven to be an oversimplification by actual thread analyses: While flames appeared within highly interactive structures, perfectly appropriate behavior could also be spotted in threads with hardly any interaction. 



\section{CHAPTER 9}

\section{Conclusion}

This study intended to merge two fields of research, interpersonal pragmatics and computer-mediated communication. With respect to the latter, we concentrated on one form of communication among many others in CMC, viz. message boards, which so far have not attracted as much scientific attention as related communicative platforms such as chat or email - especially not in combination with the first field of research mentioned above. In terms of interpersonal pragmatics, it was vitally important to this study to cover the whole range of interpersonal relations, i.e. the positive as well as the negative sides of human behavior towards each other and not just to focus on one particular, isolated phenomenon.

The objective of this study has been twofold: We tried to shed some light on the basic question of how interpersonal relations are established, managed and negotiated in online message boards. At the same time, we kept in view a common myth about online communication in general. In concentrating on the message board system The Student Room (TSR) as one representative of message boards, this study tried to falsify the claim that online communication is unsuited for interpersonal purposes and a hotbed for uninhibited and rude behavior. In doing just that, the study showed that interpersonal relations in online message boards should be judged very carefully as they are highly context-, user- and even topic-sensitive entities. In order to outline the most important pitfalls and insights for such an analysis and to summarize the final results of this investigation, the research questions presented in Chapter 1 will serve as a guideline:

1. What are the technological and social prerequisites and mechanisms of communication in online message boards like The Student Room (TSR)? How are they made use of?

As could be shown in Chapter 2, users have a lot of template-based and textual options at their disposal, through which they can convey propositional as well as interpersonal contents in and outside of their posts. Message boards try to recreate mechanisms usually available in face-to-face communication - at least to a certain degree by means of compensatory mechanisms. For that reason, participants do have (an equivalent of) para- and nonverbal clues at hand and do have access to contextual input automatically disclosed by the message board system 
and individually by their interlocutors. In this sense, the oft-cited "dramaturgical weakness" of a "faceless" form of communication can indeed be reduced.

The analysis of 50 threads revealed that message board participants made regular use of some of said compensatory mechanisms, while neglecting others. Although their usage surely depends on highly idiosyncratic choices, all posts resorted to one mechanism or the other. Profiles and signatures, to give two examples for template-based compensatory mechanisms, were sometimes crammed with personal details, information about likes and dislikes and instructions for interlocutors among many others. In other cases, however, they were practically non-existent. Entries, on the other hand, saw the change of font sizes and colors for the expression of emotional states and attitudes quite regularly, while emotes almost seemed to have gone out of style. For this purpose, smileys presented themselves as ubiquitous, multifunctional components of message board entries. Even the multimodal nature of message boards was exploited quite frequently to make a statement with links and embedded objects instead of phrasing sentences. All of this goes to show that although the means may be slightly different when compared to face-to-face contexts, users are, in fact, given strategies which they use to communicate their emotions, their relations and their face claims - in a word, to be virtually "co-present".

Owed to the fragmentary display of interlocutors' personal backgrounds, interpersonal roles need to be redefined and renegotiated constantly in message board exchanges (see Chapter 3). Although some experienced regulars have taken over responsibilities within this community of practice (for example as moderators and PS helpers), the analysis has exposed them to behave just as (in)conspicuous as any other member. Only in some cases did their behavior mirror their elevated status within the group.

2. How can key (as well as marginal) concepts of interpersonal relations be defined and clustered adequately, especially in view of analyzing online communication in message boards?

Basically, users' expectations and aims when participating in a message board such as TSR can be broken down to sharing and being part of a group. It became obvious that authors of posts do not set aside these basic human needs just because they decided to communicate in a mediated context. Thus, borrowing from theories that were originally designed for face-to-face encounters in order to explain mediated communication was only the next logical step in this study.

Based on critical reviews of classical and postmodern approaches to politeness and impoliteness, two modified frameworks could be developed: the combined model of face and facework (following Goffman 1967 and Brown/Levinson 
1978/1987) as well as the revised model of relational work (based on Locher/Watts 2005). The advantage of the first model, in comparison to its two predecessors, lies in the combination of both readings of the notion of face, which are in my opinion equally valuable: face as a conglomerate of basic human needs (association and dissociation) and face as a discursive process of negotiation. The second model needed to be extended slightly in order for it to account for the entire panoply of relational work: unmarked/appropriate behavior, politeness, impoliteness (including rudeness), but above that also mock-impoliteness and mock-politeness. Locher/ Watts' integrative perspective was insofar advantageous as it allowed for the direct comparison of and differentiation between all these types of relational work. In this way, their model also proved to be helpful for the definition of its notoriously fuzzy components, with politeness leading the way. After all, every one of the above notions is an unstable and evaluative construct, which lies in the eyes of the participating and/or observing beholders. Both frameworks set the agenda for the empirical analysis and could be applied successfully to actual data.

3. Which contextual factors need to be included when evaluating users' online behavior? Where can we find them in the message board system of The Student Room (TSR)?

In the evaluation of (online) behavior, contextual factors cannot be bracketed out. For this reason, Chapter 6 summarized (personal and interpersonal) contextual as well as (extra-)medial environmental factors. Knowing where to look for these clues in message boards can thus be very profitable, also with regard to users' face claims, which can be read out of a whole range of templates and can sometimes be traced within entire online appearances. Accordingly, checking signatures, profile information, the number of posts, labels and most of all the reputation system (including the "warnings levels") has proven to be extremely insightful in order to find out how members want to be perceived by others. Above that, they may also reveal the outcome of preceding encounters, i.e. earlier attempts at facework and relational work. In this regard, contextual factors in the broadest sense can help explain interlocutors' assessments of the situation and can come in handy in cases of ambiguous evaluations.

4. How are interpersonal relations formally expressed and interactively negotiated by experienced and new message board members? How many incidences of (the various types of) positively and negatively marked behavior can be found?

The last of the research questions was answered in the analytical part of this study. In successfully avoiding mistakes of previous studies, the present analysis 
- worked empirically on the problem;

- concentrated on one form of communication within CMC in order not to measure all of them with the same yardstick;

- worked beyond the sentence level, i.e. focused on complete utterances or even series of moves and counter-moves;

- distinguished more carefully among different users, the relationship of group members, the personalities of individual members and other contextual factors;

- allocated user perceptions a central position in the evaluation of behavior;

- investigated interaction from a participant observer's perspective also, as interlocutors' reactions, explicit ones in particular, were not always available.

Chapter 7 put special emphasis on the first posts and their authors, the thread starters, as they were suspected to set the course for the entire thread - formally as well as interpersonally. This turned out to be a correct estimation: Once a certain topic was proposed, be it in an aggressive or altruistic manner, reactions mirrored the underlying emotional stance and interpersonal attitude in most cases reliably. Even thread starters showed awareness for the importance of their opening posts, which is why some of these initial contributions were rephrased by their originators almost immediately after their first publication to avoid anticipated misunderstandings and friction.

Both Chapters 7 and 8 examined facework qualitatively and listed members' strategies, always adorned with a selection of illustrative examples. Not only did they exhibit the full range of participants' creativity to make their feelings and attitudes towards fellow-users and their opinions known. Above that, we could also detect message board specific strategies to accomplish this purpose, such as giving reputation to others by "repping" them or by joining "societies" in order to signal association, to name but two.

Chapter 8 also saw the quantitative analysis of relational work for all 50 threads, which proved that the lion's share of behavior is indeed appropriate. Comparatively few instances of inappropriate behavior, with some flames far and few between showed that in most cases, interlocutors do not go online to pick a fight but to find like-minded others and to enjoy their company. What is more, the classification of threads according to their communicative purpose also supports the conclusion that interpersonal behavior is not only dependent on the context and the users but also largely topic-driven. At times, purely phatic communication could be witnessed, even containing passages of mutual flattering. With a view to these results, at least this particular message board has proven definitely suitable for interpersonal purposes. To this end, the category of appropriate/unmarked behavior was necessary and useful to represent online behavior in a much 
more nuanced and realistic way. The same holds true for another novelty in the course of action of the empirical analysis, namely the functional differentiation of posts depending on whether they are evaluated by interlocutors' subsequent posts or not (type A and type B posts) and the separation of those posts which do not refer to any particular, preceding post at all (type $\mathrm{C}$ posts).

More often than not, the conversational flow runs smoothly and does not exhibit any amplitudes to either side, be it in the form of positively or negatively marked behavior. Yet still message boards and related types of CMC have a bad record (see above). How is this possible, especially in the light of these results? As most of the functional behavior in this corpus is clearly "unmarked", it usually drifts by almost unnoticed. Consequently, the conversation seems uneventful in terms of relational work, although it should be labeled "appropriate" instead. More noticeable, however, are those communicative events which are positively marked and - to an even higher degree - those which are strikingly negatively marked and thus dysfunctional (see the high percentages scored for rudeness in general and for flames in particular). In this context, it should also be added that marked behavior, be it positively or negatively, shows the tendency to be carried to the extremes in message boards, which is why politeness always seems more heartfelt and abundant, impoliteness, on the other hand, extremely outspoken and drastic. Those are the ones to clearly stand out against the rest, which makes counting them so much easier. I argue that it is due to this misperception that online communication, including message board interaction, has wrongfully earned labels such as "uninhibited", "unfriendly" and "rude". Although the figures of this analysis support this hypothesis, they certainly cannot confirm it for good. For that reason, I would like to make a case for more qualitative and, wherever possible, also quantitative research on a diversified range of message boards. Even if this course of action brings to light limited snapshots of interpersonal microspheres, we can still gain more insights into this intriguing field of research by adopting a comparative perspective. 



\section{Bibliography}

Anderson, Benedict. 1983. Imagined Societies: Reflections on the Origin and Spread of nationalism. London: Verso.

Androutsopoulos, Jannis K. 2006. "Introduction: Sociolinguistics and Computer-Mediated Communication”. Journal of Sociolinguistics 10 (4): 419-438.

Androutsopoulos, Jannis K., Runkehl, Jens, Schlobinski, Peter and Siever, Torsten (eds). 2006. Neuere Entwicklungen in der linguistischen Internetforschung. Hildesheim: Olms.

Arndt, Horst and Janney, Richard. ${ }^{2} 2005$, 1992. "Intracultural Tact versus Intercultural Tact". In Politeness in Language: Studies in its History, Theory and Practice, Richard J. Watts, Sachiko Ide and Konrad Ehlich (eds), 21-41. Berlin: Mouton de Gruyter.

Arundale, Robert B. 2006. "Face as Relational and Interactional: A Communication Framework for Research on Face, Facework, and Politeness". Journal of Politeness Research 2: 193-216.

Arundale, Robert B. 2010. "Constituting Face in Conversation: Face, Facework and Interactional Achievement". Journal of Pragmatics 42: 2078-2105.

Asteroff, Janet F. 1987. Paralanguage in Electronic Mail: A Case Study. Columbia University/NY: $\mathrm{PhD}$ Thesis.

Auer, Peter. 1995. “Context and Contextualization”. In Handbook of Pragmatics, Jef Verschueren et al. (eds), 1-19. Amsterdam/Philadelphia: John Benjamins.

Austin, Paddy. 1990. "Politeness Revisited - the Dark Side". In New Zealand Ways of Speaking English, Allan Bell and Janet Holmes (eds), 277-293. Philadelphia: Multilingual Matters Ldt.

Avgerinakou, Anthi. 2003. “Flaming' in Computer-Mediated Interaction”. In Rethinking Communicative Interaction, Colin B. Grant (ed.), 273-293. Amsterdam/Philadelphia: John Benjamins.

Baker, Paul. 2001. "Moral Panic and Alternative Identity Construction in Usenet". Journal of Computer-Mediated Communication 7 (1). http://jcmc.indiana.edu/vol7/issue1/baker. html, July 5th 2012.

Bargiela-Chiappini, Francesca. 2003. "Face and Politeness: New (Insights) for Old (Concepts)". Journal of Pragmatics 35 (10, 11): 1453-1469.

Baron, Naomi. 1984. "Computer Mediated Communication as a Force in Language Change". Visible Language 32: 118-141.

Baym, Nancy K. 1995. “The Emergence of Community in Computer-Mediated Communication". In CyberSociety: Computer-Mediated Communication and Community, Steven G. Jones (ed.), 138-163. Thousand Oaks/CA: Sage.

Baym, Nancy K. 1997. "Interpreting Soap Operas and Creating Community: Inside an Electronic Fan Culture". In Culture of the Internet, Sara Kiesler (ed.), 103-120. Mahwah/NJ: Lawrence Erlbaum. 
Baym, Nancy K. 1998. “The Emergence of Online Community”. In CyberSociety 2.0: Revisiting Computer-Mediated Communication and Community, Steven G. Jones (ed.), 35-68. Thousand Oaks/CA: Sage.

Baym, Nancy K. 2000. Tune In, Log On: Soaps, Fandom, and Online Community. Thousand Oaks/CA: Sage.

Bazzanella, Carla. 2002. "The Significance of Context in Comprehension: The We Case”. In Contact in Context: Special Issue of Foundations of Science 7 (3), Bruce Edmonds and Varol Akman (eds), 239-254. Dordrecht: Kluwer Academic Publishers.

Beebe, Leslie M. 1995. "Polite Fictions: Instrumental Rudeness as Pragmatic Competence”. In Georgetown University Round Table on Language and Linguistics 1995. Linguistics and the Education of Language Teachers: Ethnolinguistic, Psycholinguistic, and Sociolinguistics Aspects, James E. Alatis, Carolyn A. Straehle, Brent Gallenberger and Maggie Ronkin (eds), 154-168. Washington D.C.: Georgetown University Press.

Beißwenger, Michael. 2002. "Das Knistern zwischen den Zeilen: Inszenierungspotenziale in der schriftbasierten Chat-Kommunikation”. http://dichtung-digital.mewi.unibas.ch/2002/ modemfieber/beisswenger.htm, July 5th 2012.

Beißwenger, Michael. 2003. "Sprachhandlungskoordination im Chat”. Zeitschrift für germanistische Linguistik 31 (2): 198-231.

Beißwenger, Michael. 2010. “Chattern unter die Finger geschaut: Formulieren und Revidieren bei der schriftlichen Verbalisierung in synchroner internetbasierter Kommunikation”. In Nähe und Distanz im Kontext variationslinguistischer Forschung, Vilmos Ágel and Mathilde Hennig (eds), 247-294. Berlin: Mouton de Gruyter.

Beißwenger, Michael, Hoffmann, Ludger and Storrer, Angelika (eds). 2004. Internetbasierte Kommunikation. Oldenburg: OBST 68.

Bell, David 2000. “Cybercultures Reader. A User's Guide”. In The Cybercultures Reader, David Bell and Barbara M. Kennedy (eds), 1-12. London: Routledge.

Benwell, Bethan and Stokoe, Elizabeth. 2006. Discourse and Identity. Edinburgh: Edinburgh Press.

Bins, Elmar and Piwinger, Boris-A. 1997. Newsgroups. Weltweit diskutieren. Bonn: Internat. Thomson Publ.

Blum-Kulka, Shoshana. 1985. "Modifiers as Indicating Devices: The Case of Requests". Theoretical Linguistics 12 (2-3): 213-229.

Blum-Kulka, Shoshana. ${ }^{2} 2005,1992$. “The Metapragmatics of Politeness in Israeli Society”. In Politeness in Language: Studies in its History, Theory and Practice, Richard J. Watts, Sashiko Ide and Konrad Ehlich (eds), 255-280. Berlin: Mouton de Gruyter.

Bolter, Jay D. 1991. Writing Space: The Computer, Hypertext, and the History of Writing. Hillsdale/NJ: Lawrence Erlbaum.

Bolter, Jay D. 2001. Writing Space: Computers, Hypertext, and the Remediation of Print. Mahwah/NJ: Lawrence Erlbaum.

Bousfield, Derek. 2008. Impoliteness in Interaction. Amsterdam/Philadelphia: John Benjamins.

Brown, Penelope. 2001. "Politeness and Language". In The International Encyclopedia of the Social and Behavioral Sciences 17, Neil J. Smelser and Paul B. Baltes (eds), 11620-11624. Oxford, UK: Elsevier.

Brown, Penelope and Levinson, Stephen C. 1978. "Universals in Language Usage: Politeness Phenomena”. In Questions and Politeness, Esther N. Goody (ed.), 56-289. Cambridge: Cambridge University Press. 
Brown, Penelope and Levinson, Stephen C. 1987. Politeness: Some Universals in Language Usage. Cambridge: Cambridge University Press.

Brown, Roger and Gilman, Albert. 1972. “The Pronouns of Power and Solidarity”. In Language and Social Context, Pier P. Giglioli (ed.), 252-282. New York: Penguin.

Brown, Gillian and Yule, George. 1983. Discourse Analysis. Cambridge: Cambridge University Press.

Bublitz, Wolfram. 2003. "Emotive Prosody: How Attitudinal Frames Help Construct Context”. In Anglistentag 2002 Bayreuth, Proceedings, Ewald Mengel, Hans-Jörg Schmid and Michael Steppat (eds), 381-391. Trier: WVT.

Bublitz, Wolfram. 2005. “The User as 'Cyberego': Text, Hypertext and Coherence”. In Anglistentag 2004 Aachen, Proceedings, Lilo Moessner and Christa M. Schmidt (eds), 311-324. Trier: WVT.

Bublitz, Wolfram. 2008. "Sailing the Islands or Watching from the Dock': The Treacherous Simplicity of a Metaphor. How We Handle 'New (Electronic) Hypertext' versus 'Old (Printed) Text”'. In Language, People, Numbers: Corpus Linguistics and Society, Andrea Gerbig and Oliver Mason (eds), 249-273. Amsterdam: Rodopi.

Bublitz, Wolfram. ${ }^{2} 2009$. Englische Pragmatik: Eine Einführung. Berlin: Erich Schmidt Verlag.

Burger, Harald. 2005. Mediensprache: Eine Einführung in Sprache und Kommunikationsformen der Massenmedien. Berlin: Mouton de Gruyter.

Burnett, Robert and Marshall, P. David. 2003. Web Theory: An Introduction. London: Routledge.

Bush, Vannevar. 1945. “As We May Think”. Atlantic Monthly. http://www.theatlantic.com/ magazine/archive/1969/12/as-we-may-think/3881/, July 5th 2012.

Caffi, Claudia. 1993. "Metapragmatics". In The Encyclopedia of Language and Linguistics, Ronald E. Asher and J. M. Y. Simpson (eds), 2461-2466. Oxford: Pergamon.

Chandler, Daniel. 1995. “Technological or Media Determinism”. http://www.aber.ac.uk/media/ Documents/tecdet/, July 5th 2012.

Chandler, Daniel. 1998. "Writing Oneself in Cyberspace". http://www.aber.ac.uk/media/ Documents/short/homepgid.html, July 5th 2012.

Cherny, Lynn. 1999. Conversation and Community: Chat in a Virtual World. Stanford/CA: Center for the study of language and information.

Claridge, Claudia. 2007. "Constructing a Corpus from the Web: Message Boards". In Corpus Linguistics and the Web, Marianne Hundt, Nadja Nesselhauf and Carolin Biewer (eds), 87-108. Amsterdam/New York: Rodopi.

Clark, Herbert H. 1982. "The Relevance of Common Ground: Comments on Sperber and Wilson's Paper”. In Mutual Knowledge, Neilson V. Smith (ed.), 124-127. London: Academic Press.

Clark, Herbert H. 2006. "Context and Common Ground”. In Encyclopedia of Language and Linguistics, Edward K. Brown (ed.), 105-108. Amsterdam: Elsevier.

Clyne, Michael. 1994. Intercultural Communication at Work: Cultural Values in Discourse. Cambridge: Cambridge University Press.

Collins, Mauri P. 1992. "Flaming: The Relationship between Social Context Cues and Uninhibited Verbal Behaviour in Computer-Mediated Communication”. http://www.mediensprache.net/archiv/pubs/2842.htm, July 5th 2012. 
Constantin, Corina, Kalyanaraman, Sriram, Stavrositu, Carmen and Wagoner, Nathan. 2002. “To Be or not to Be Emotional". Paper presented at the Communication Technology and Policy Division at the 85th Annual Convention of the Association for Education in Journalism and Mass Communication (AEJMC), Miami, Florida.

Coupland, Nikolas and Jaworski, Adam. 2004. "Sociolinguistic Perspectives on Metalanguage: Reflexivity, Evaluation and Ideology”. In Metalanguage: Social and Ideological Perspectives, Adam Jaworski, Nikolas Coupland and Dariusz Galasinski (eds), 15-52. Berlin: Mouton de Gruyter.

Crystal, David. 2001. Language and the Internet. Cambridge: Cambridge University Press.

Culnan, Mary J. and Markus, M. Lynne. 1987. “Information Technologies". In Handbook of Organizational Communication: An Interdisciplinary Perspective, Frederic M. Jablin, Linda L. Putnam, Karlene H. Roberts and Lyman W. Porter (eds), 420-443. Newbury Park/CA: Sage.

Culpeper, Jonathan. 1996. “Towards an Anatomy of Impoliteness”. Journal of Pragmatics 25 (3): 349-367.

Culpeper, Jonathan, Bousfield, Derek and Wichmann, Anne. 2003. "Impoliteness Revisited: with Special Reference to Dynamic and Prosodic Aspects". Journal of Pragmatics 35 (10, 11): 1545-1579.

Cupach, William R. and Metts, Sandra. 1994. Facework. Thousand Oaks/CA: Sage.

Darics, Erika. 2010. “Politeness in Computer-Mediated Discourse of a Virtual Team”. Journal of Politeness Research 6: 129-150.

de Beaugrande, Robert-Alain and Dressler, Wolfgang U. 1981. Einführung in die Textlinguistik. Tübingen: Niemeyer.

December, John. 1997. “Notes on Defining Computer-Mediated Communication”. CMC Magazine. http://www.december.com/cmc/mag/1997/jan/december.html, July 5th 2012.

Derks, Daantje, Fischer, Agneta H. and Bos, Arjan E. R. 2008. "The role of emotion in computer-mediated communication: A review”. Computers in Human Behavior 24 (3): 766-785.

Derlega, Valerian J. and Chaikin, Alan. L. 1977. "Privacy and Self-Disclosure in Social Relationships”. Journal of Social Issues 33 (3): 102-115.

Döring, Nicola. 1999. “Zwischenmenschliche Nähe im Internet”. In Konnekt: Das Medienpaket, Dieter Glaap and Jürgen Ertelt (eds), 37-45. Remscheid: Akademie Remscheid.

Donath, Judith S. 1999. "Identity and Deception in the Virtual Community". In Communities in Cyberspace, Marc A. Smith and Peter Kollock (eds), 29-59. London: Routledge.

Donnerhacke, Lutz. 1996. "Usenet: Die Einrichtung von Diskussionsforen”. In Die Netz-Revolution: Auf dem Weg in die Weltgesellschaft, Martin Rost (ed.), 70-75. Frankfurt/Main: Eichborn.

Douglas, Karen M. and McGarty, Craig. 2001. "Identifiability and Self-Presentation: ComputerMediated Communication and Intergroup Interaction". British Journal of Social Psychology 40 (3): 399-416.

Dürscheid, Christa. 1999. "Zwischen Mündlichkeit und Schriftlichkeit: die Kommunikation im Internet”. Papiere zur Linguistik 60 (1): 17-30.

Dürscheid, Christa. 2003. "Medienkommunikation im Kontinuum zwischen Mündlichkeit und Schriftlichkeit: Theoretische und empirische Probleme". Zeitschrift für Angewandte Linguistik 38: 37-56.

Dürscheid, Christa. 2006. Einführung in die Schriftlinguistik: Ergänzt um ein Kapitel zur Typographie von Jürgen Spitzmüller. Göttingen: Vandenhoeck and Ruprecht. 
Durkheim, Emile. 1915. The Elementary Forms of the Religious Life. London: George Allen and Unwin Ltd.

Eckert, Penelope. 2006. “Communities of Practice”. In Encyclopedia of Language and Linguistics, Edward K. Brown (ed.), 683-685. Amsterdam: Elsevier.

Eckert, Penelope and McConnell-Ginet, Sally. 1998. "Communities of Practice: Where Language, Gender and Power all Live”. In Language and Gender, Jennifer Coates (ed.), 484494. Oxford: Blackwell.

Edinger, Joyce A. and Patterson, Miles L. 1983. "Nonverbal Involvement and Social Control". Psychological Bulletin 93: 30-56.

Eelen, Gino. 2001. A Critique of Politeness Theories. Manchester: St. Jerome Publ.

Ehlich, Konrad. 22005, 1992. "On the Historicity of Politeness". In Politeness in Language: Studies in its History, Theory and Practice, Richard J. Watts, Sachiko Ide and Konrad Ehlich (eds), 71-107. Berlin: Mouton de Gruyter.

Eisenlauer, Volker and Hoffmann, Christian. 2008. "The Metapragmatics of Remediated Text Design”. Information Design Journal 16(1): 1-18.

Erickson, Thomas. 1996. “The World Wide Web as Social Hypertext”. http://www.pliant.org/ personal/Tom_Erickson/SocialHypertext.html, July 5th 2012.

Fabrot, Bernard. 2001. Newsgroups: The.little.Internet.guide. London: Cassell Illustrated.

Feldman, Martha S. 1987. "Electronic Mail and Weak Ties in Organizations". Office: Technology and People 3: 83-101.

Fernback, Jan and Thompson, Brad. 1995. "Virtual Communities: Abort, Retry, Failure?". http://www.well.com/user/hlr/texts/VCcivil.html, July 5th 2012.

Ferrara, Kathleen, Brunner, Hans and Whittemore, Greg. 1991. "Interactive Written Discourse as an Emergent Register". Written Communication 8: 8-34.

Firth, John R. 1957. Papers in Linguistics 1934-1951. London: Oxford University Press.

Foster, Derek. 1997. "Community and Identity in the Electronic Village". In Internet Culture, David Porter (ed.), 23-37. London: Routledge.

Foucault, Michel. 1980. Power/Knowledge: Selected Interviews and Other Writings. 1972-1977. Brighton: Harvester Press.

Foucault, Michel. 1988. “Technologies of the Self”. In Technologies of the Self, Luther H. Martin, Huck Gutman and Patrick H. Hutton (eds), 16-49. Amherst: University of Massachusetts Press.

Fraser, Bruce. 1980. “Conversational Mitigation”. Journal of Pragmatics 4: 341-350.

Fraser, Bruce. 1990. "Perspectives on Politeness”. Journal of Pragmatics 14 (2): 219-236.

Fraser, Bruce. 1999. “Whither Politeness?”. Paper presented at the International Symposium on Linguistic Politeness: Theoretical Approaches and Intercultural Perspective, Chulalongkorn University, Bangkok, Thailand.

Giddens, Anthony. 1991. Modernity and Self-Identity: Self and Society in the Late Modern Age. Stanford: Stanford University Press.

Gimenez, Martha E. 1997. “The Dialectics between the Real and the Virtual”. In Mapping Cyberspace: Social Research on the Electronic Frontier, Joseph E. Behar (ed.), 79-91. Oakdale/ NY: Dawling College Press.

Goffman, Erving. 1955. "On Face-Work: An Analysis of Ritual Elements in Social Interaction”. Psychiatry 18: 213-231.

Goffman, Erving. 1967. Interaction Ritual: Essays on Face-to-Face Behaviour. Harmondsworth: Penguin Books. 
Goffman, Erving. 1981. Forms of Talk. Oxford: Blackwell.

Goodwin, Charles and Duranti, Alessandro. 1992. "Rethinking Context: an Introduction". In Rethink Context: Language as an Interactive Phenomenon, Alessandro Duranti and Charles Goodwin (eds), 1-42. Cambridge: Cambridge University Press.

Graham, Sage L. 2008. "A Manual for (Im)Politeness?: The Impact of the FAQ in an Electronic Community of Practice". In Impoliteness in Language: Studies on its Interplay with Power in Theory and Practice, Miriam A. Locher and Derek Bousfield (eds), 281-304. Berlin: Mouton de Gruyter.

Graham, Sage L. 2010. “THAT’S HOW WE ROLE: The Establishment of Functional Roles vs. Idealized Identities in an Online Community of Practice". Paper presented at the 5th International Symposium on Politeness, Basel/Swiss.

Grice, Herbert P. 1975. "Logic and conversation". In Syntax and Semantics 3, Peter Cole and Jerry L. Morgan (eds), 41-58. Speech Acts. New York et al.: Academic Press.

Grimshaw, Allen D. 1990. Conflict Talk: Sociolinguistic Investigations of Arguments in Conversations. Cambridge: Cambridge University Press.

Gumperz, John J. 1992. "Contextualization and Understanding”. In Rethink Context: Language as an Interactive Phenomenon, Alessandro Duranti and Charles Goodwin (eds), 229-251. Cambridge: Cambridge University Press.

Haferland, Harald and Paul, Ingwer. 1996. "Eine Theorie der Höflichkeit". Oldenburg: OBST 52: 7-69.

Halliday, Michael A. K. 1978. Language as Social Semiotic: The Social Interpretation of Language and Meaning. Baltimore: University Park Press.

Harrison, Sandra. 2000. "Maintaining the Virtual Community: Use of Politeness Strategies in an Email Discussion Group". In Words on the Web: Computer Mediated Communication, Lyn Pemberton and Simon Shurville (eds), 69-78. Exeter/Portland: Intellect Books.

Hatipoglu, Çiler. 2007. “(Im)Politeness, National and Professional Identities and Context: Some Evidence from E-mailed 'Call for Papers”'. Journal of Pragmatics 39 (4): 759-773.

Haugh, Michael. 2007. “The Discursive Challenge to Politeness Research: An Interactional Alternative". Journal of Politeness Research 3: 295-317.

Held, Gudrun. 1995. Verbale Höflichkeit: Studien zur linguistischen Theoriebildung und empirische Untersuchung zum Sprachverhalten französischer und italienischer Jugendlicher in Bittund Dankessituationen. Tübingen: Narr.

Held, Gudrun. ${ }^{2} 2005,1992$. "Politeness in Linguistic Research". In Politeness in Language, Richard J. Watts, Sachiko Ide and Konrad Ehlich (eds), 131-153. Berlin: Mouton de Gruyter.

Herring, Susan. 1995. "Politeness in Computer Culture: Why Women Thank and Men Flame”. In Communicating in, through, and across Cultures: Proceedings of the Third Berkeley Women and Language Conference, Mary Bucholtz, A. C. Liang, Laurel A. Sutton and Caitlin Hines (eds), 278-294. Berkeley: Berkeley Women and Language Group.

Herring, Susan (ed.). 1996. Computer-Mediated Communication: Linguistic, Social and CrossCultural Perspectives. Amsterdam/Philadelphia: John Benjamins.

Herring, Susan. 1999. "Interactional Coherence in CMC". Journal of Computer-Mediated Communication 4 (4) (Special Issue on Persistent Conversation). http://jcmc.indiana.edu/vol4/ issue4/herring.html, July 5th 2012.

Herring, Susan. 2001. "Computer-Mediated Discourse”. In The Handbook of Discourse Analysis, Deborah Schiffrin, Deborah Tannen and Heidi E. Hamilton (eds), 612-634. Oxford: Blackwell. 
Herring, Susan. 2004. "Computer-Mediated Discourse Analysis: An Approach to Researching Online Behavior". In Designing for Virtual Communities in the Service of Learning, Sasha A. Barab, Rob Kling and James H. Gray (eds), 338-376. New York: Cambridge University Press.

Herring, Susan. 2007. "A Faceted Classification Scheme for Computer-Mediated Discourse". Language@Internet 4/2007. http://www.languageatinternet.org/articles/2007/761, July 5th 2012.

Herring, Susan, Job-Sluder, Kirk, Scheckler, Rebecca and Barab, Sasha. 2002. "Searching for Safety Online: Managing 'Trolling' in a Feminist Forum”. The Information Society 18 (5): 371-384.

Hiltz, Starr R., Johnson, Kenneth and Turoff, Murray. 1986. "Experiments in Group Decision Making: Communication Process and Outcome in Face-to-face versus Computerized Conferences”. Human Communication Research 13: 225-252.

Hiltz, Starr R. and Turoff, Murray. 1981. "The Evolution of User Behavior in a Computerized Conferencing System”. Communications of the ACM 24: 739-762.

Ho, David Y. 1994. “Face Dynamics: From Conceptualization to Measurement”. In The Challenge of Facework: Cross-Cultural and Interpersonal Issues, Stella Ting-Toomey (ed.), 3-13. Albany/NY: State University of New York Press.

Hobbs, Pamela. 2003. “The Medium is the Message: Politeness Strategies in Men's and Women's Voice Mail Messages". Journal of Pragmatics 35: 243-262.

Hoffmann, Christian. 2012. Cohesive Profiling: Meaning and Interaction in Personal Weblogs. Amsterdam/Philadelphia: John Benjamins.

Holly, Werner. 1979. Imagearbeit in Gesprächen: Zur linguistischen Beschreibung des Beziehungsaspekts. Tübingen: Niemeyer.

Holly, Werner. 1997. "Zur Rolle von Sprache in Medien: Semiotische und kommunikationsstrukturelle Grundlagen”. Muttersprache 1: 64-75.

Holmberg, Anna. 2008. The Power of Insults: A Study of Condescending Linguistic Strategies in four English Online Discussions. Växjö Univeristet: B.A. Thesis.

Holmes, Janet. 1995. Women, Men and Politeness. London: Longman.

Holmes, Janet. 2006. “Politeness Strategies As Linguistic Variables”. In Encyclopedia of Language and Linguistics, Edward K. Brown (ed.), 684-697. Amsterdam: Elsevier.

Holtgraves, Thomas. 2005. "Social Psychology, Cognitive Psychology, and Linguistic Politeness”. Journal of Politeness Research 1 (1): 73-93.

Hongladarom, Krisadawan. 2007. “'Don't Blame me for Criticizing you ...': A Study of Metapragmatic Comments in Thai”. In Metapragmatics in Use, Wolfram Bublitz and Axel Hübler (eds), 29-47. Amsterdam/Philadelphia: John Benjamins.

$\mathrm{Hu}, \mathrm{Hsien}$ C. 1944. “The Chinese Concepts of 'Face”. American Anthropologist 46: 45-64.

Huber, Oliver. 2000. Hyper-Text-Linguistik: TAH: Ein textlinguistisches Analysemodell für Hypertexte. Theoretisch und praktisch exemplifiziert am Problemfeld der typisierten Links von Hypertexten im World Wide Web. Ludwig-Maximilians-Universität München: PhD Thesis. http://edoc.ub.uni-muenchen.de/921/1/Huber_Oliver.pdf, July 5th 2012.

Humboldt, Wilhelm v. 1820, 1946. Über das vergleichende Sprachstudium in Beziehung auf die verschiedenen Epochen der Sprachentwicklung. Leipzig: Meiner.

Hymes, Dell. 1974. Foundations of Sociolinguistics: An Ethnographic Approach. Philadelphia: University of Pennsylvania Press. 
Hymes, Dell. 1986. "Models of Interaction of Language and Social Life". In Directions in Sociolinguistics: The Ethnography of Communication, John J. Gumperz and Dell Hymes (eds), 35-71. Oxford: Blackwell.

Jasper, Dirk. 1997. Internet Newsgroups: Suchen, Anzapfen, Nutzen, Diskutieren. Düsseldorf: Econ $\mathrm{Tb}$.

Johansen, Robert, Vallee, Jacques and Spangler, Kathleen. 1988. “Teleconferencing: Electronic Group Communication”. In Small Group Communication: A Reader, Robert S. Cathcart and Larry A. Samovar (eds), 140-154. Dubuque/IA: William C. Brown.

Johnstone, Barbara and Bean, Judith M. 1997. "Self Expression and Linguistic Variation”. Language in Society 26 (2): 221-246.

Joinson, Adam. 1998. "Causes and Implications of Disinhibited Behavior on the Internet". In Psychology and the Internet: Intrapersonal, Interpersonal and Transpersonal Implications, Jane Gackenbach (ed.), 43-58. San Diego: Academic Press.

Jones, Rodney H. 2002. “The Problem of Context in Computer Mediated Communication”. Paper presented at the Georgetown Roundtable on Language and Linguistics, March 7-9 2002. http://personal.cityu.edu.hk/ enrodney/Research/ContextCMC.doc, July 5th 2012.

Jones, Steven G. 1995. "Understanding Community in the Information Age". In CyberSociety, Computer-Mediated Communication and Community, Steven G. Jones (ed.), 10-35. Thousand Oaks/CA: Sage.

Jones, Steven G. 2002. "The Internet Goes to College: How Students are Living in the Future with Today's Technology”. http://www.pewinternet.org/ /media//Files/Reports/2002/ PIP_College_Report.pdf.pdf, July 5th 2012.

Kang, Jerry. 2000. “Cyber-Race”. Harvard Law Review 113: 1130-1208.

Kasper, Gabriele. 1990. "Linguistic Politeness: Current Research Issues”. Journal of Pragmatics 14 (2): 193-218.

Kasper, Gabriele. 1997. "Linguistic Etiquette". In The Handbook of Sociolinguistics, Florian Coulmas (ed.), 374-385. Oxford: Blackwell.

Katz, James E., Rice, Ronald E. and Aspden, Philip. 2001. “The Internet”. American Behavioral Scientist 45: 405-419.

Kendall, Lori. 1999. "Recontextualizing 'Cyberspace’: Methodological Considerations for Online Research". In Doing Internet Research: Critical Issues and Methods for Examining the Net, Steven G. Jones (ed.), 57-74. Thousand Oaks/CA: Sage.

Kienpointner, Manfred. 1997. "Varieties of Rudeness: Types and Functions of Impolite Utterances". Functions of Language 4 (2): 251-287.

Kiesler, Sara, Siegel, Jane and McGuire, Timothy W. 1984. "Social Psychological Aspects of Computer-mediated Communication". American Psychologist 39: 1123-1134.

Kollock, Peter and Smith, Marc A. 1996. "Managing the Virtual Commons: Cooperation and Conflict in Computer Communities". In Computer-Mediated Communication: Linguistic, Social and Cross-cultural Perspectives, Susan Herring (ed.), 109-128. Amsterdam/Philadelphia: John Benjamins.

Kollock, Peter and Smith, Marc A. 1999. "Communities in Cyberspace”. In Communities in Cyberspace, Marc A. Smith and Peter Kollock (eds), 3-25. London: Routledge.

Kuhlen, Rainer. 1991. Hypertext. Berlin: Springer.

Labov, William. 1972. Language in the Inner City: Studies in Black English Vernacular. Philadelphia: University of Pennsylvania Press.

Lachenicht, Lance G. 1980. "Aggravating Language: A Study of Abusive and Insulting Language”. Papers in Linguistics: International Journal in Human Communication 13 (4): 607-687. 
Lakoff, Robin T. 1973. “The Logic of Politeness, or Minding your p's and q's.. In Papers from the Ninth Regional Meeting of the Chicago Linguistics Society, Claudia Corum et al. (eds), 292-305. Chicago: Department of Linguistics, University of Chicago.

Lakoff, Robin. T. 1979. "Stylistic Strategies within a Grammar of Style”. In Language, Sex, and Gender: Does la Différence Make a Difference?, Judith Orasanu et al. (eds), 53-78. New York: The New York Academy of Sciences New York.

Lakoff, Robin T. and Ide, Sachiko. 2005. Broadening the Horizon of Linguistic Politeness. Amsterdam/Philadelphia: John Benjamins.

Landow, George. 1992. Hypertext: The Convergence of Contemporary Critical Theory and Technology. Baltimore: Johns Hopkins University Press.

Launspach, Sonja. 2000. "Literal or Loose Talk: the Negotiation of Meaning on an Internet Discussion List”. In Words on the Web: Computer Mediated Communication, Lyn Pemberton and Simon Shurville (eds), 87-95. Exeter/Portland: Intellect Books.

Lave, Jean and Wenger, Etienne. 1991. Situated Learning: Legitimate Peripheral Participation. Cambridge: Cambridge University Press.

Lea, Martin and Spears, Russell. 1995. "Love at first Byte? Building Personal Relationships over Computer Networks". In Understudied Relationships: Off the Beaten Track, Julia T. Wood and Steve Duck (eds), 197-233. Newbury Park/CA: Sage.

Lea, Martin, O’Shea, Tim, Fung, Pat and Spears, Russell. 1992. "'Flaming' in Computer-Mediated Communication: Observations, Explanations and Implications”. In Contexts of ComputerMediated Communication, Martin Lea (ed.), 89-112. New York: Harvester-Wheatsheaf.

Lee, Hangwoo. 2005. "Behavioral Strategies for Dealing with Flaming in an Online Forum”. The Sociological Quarterly 46 (2): 385-403.

Leech, Geoffrey N. 1983. Principles of Pragmatics. New York: Longman.

Locher, Miriam A. 2004. Power and Politeness in Action: Disagreements in Oral Communication. Berlin: Mouton de Gruyter.

Locher, Miriam A. 2006a. Advice Online. Amsterdam/Philadelphia: John Benjamins.

Locher, Miriam A. 2006b. "Polite Behavior within Relational Work: The Discursive Approach to Politeness". Multilingua: Journal of Cross-Cultural and Interlanguage Communication 25 (3): 249-267.

Locher, Miriam A. 2011. "Situated Impoliteness: The Interface between Identity Work and Relational Construction". In Situated Politeness, Bethan Davies, Michael Haugh and Andrew J. Merrison (eds), 187-208. London: Continuum.

Locher, Miriam A. and Bousfield, Derek (eds). 2010. Impoliteness in Language: Studies on its Interplay with Power in Theory and Practice. Berlin: Mouton de Gruyter.

Locher, Miriam A. and Watts, Richard J. 2005. "Politeness Theory and Relational Work". Journal of Politeness Research 1 (1): 9-33.

Locher, Miriam A. and Watts, Richard J. 2008. "Relational Work and Impoliteness: Negotiating Norms of Linguistic Behavior". In Impoliteness in Language: Studies on its Interplay with Power in Theory and Practice, Derek Bousfield and Miriam A. Locher (eds), 77-99. Berlin: Mouton de Gruyter.

Lorenzo-Dus, Nuria, Garces-Conejos Blitvich, Pilar and Bou-Franch, Patricia. 2011. "On-line Polylogues and Impoliteness: The Case of Postings Sent in Response to the Obama Reggaeton YouTube Video". Journal of Pragmatics 43: 2578-2593.

Malinowski, Bronislaw. 1922. Argonauts of the Western Pacific. London: Routledge.

Marcoccia, Michael. 2004. "On-line Polylogues: Conversation Structure and Participation Frameworks in Internet Newsgroups”. Journal of Pragmatics 36: 115-145. 
Maricic, Ibolya. 2001. “Cyberpoliteness: Requesting Strategies on the Linguist List”. In Pragmatics in 2000. Selected papers from the 7th International Pragmatics Conference. Vol. 2. Enikö T. Németh (ed.), 409-416. Antwerp: International Pragmatics Association (IPrA).

Maricic, Ibolya. 2005. Face in Cyberspace: Facework, (Im)Politeness and Conflict in English Discussion Groups. Växjö: Växjö University Press.

McCormick, Naomi B. and McCormick, John W. 1992. "Computer Friends and Foes: Content of Undergratuates' Electronic Mail”. Computers in Human Behavior 8: 379-405.

McKenna, Katelyn Y. A. and Bargh, John A. 1999. "Causes and Consequences of Social Interaction on the Internet: A Conceptual Framework”. Media Psychology 1: 249-269.

McKenna, Katelyn Y. A. and Bargh, John A. 2000. "Plan 9 from Cyberspace: The Implications of the Internet for Personality and Social Psychology". Personality and Social Psychology Bulletin 4: 57-75.

McKenna, Katelyn Y. A., Green, Amie S. and Gleason, Marci E. J. 2002. "Relationship Formation on the Internet: What's the Big Attraction?”. Journal of Social Issues 58: 9-31.

McLaughlin, Margaret L., Osborne, Kerry K. and Smith, Christine B. 1995. "Standards of Conduct on Usenet". In CyberSociety: Computer-Mediated Communication and Community, Steven G. Jones (ed.), 90-111. Thousand Oaks/CA: Sage.

McLuhan, Marshall. 1964. Understanding Media: The Extensions of Man. New York: Mentor.

Meier, Ardith J. 1995. “Defining Politeness: Universality In Appropriateness”. Language Science 17 (4): 345-356.

Meier, Ardith J. 2004. “Has 'Politeness' Outlived its Usefulness?”. Vienna English Working Papers 13 (1): 5-22. http://www.univie.ac.at/Anglistik/views/O4_1/0401ALL.PDF, July 5th 2012.

Mercer, Neil. 2000. Words and Minds: How we Use Language to Think Together. London: Routledge.

Meyrowitz, Joshua. 1985. No Sense of Place: The Impact of Electronic Media on Social Behavior. Oxford: Oxford University Press.

Mills, Sara. 2002. "Rethinking Politeness, Impoliteness and Gender Identity”. In Gender Identity and Discourse Analysis, Lia Litosseliti and Jane Sunderland (eds), 69-89. Amsterdam/Philadelphia: John Benjamins.

Mills, Sara. 2003. Gender and Politeness. Cambridge: Cambridge University Press.

Mills, Sara. 2005. "Gender and Impoliteness". Journal of Politeness Research 1: 263-280.

Morand, David A. and Ocker, Rosalie J. 2003. "Politeness Theory and Computer-Mediated Communication: A Sociolinguistic Approach to Analyzing Relational Messages". HICCS '03, 36th Annual Hawaii International Conference on System Sciences. http://www2.computer.org/portal/web/csdl/doi/10.1109/HICSS.2003.1173660, July 5th 2012.

Münz, Stefan. 2002. "Foren und Boards". http://aktuell.de.selfhtml.org/artikel/gedanken/ foren-boards/, July 5 th 2012.

Murray, Denise E. 1988. “The Context of Oral and Written Language: A Framework for Mode and Medium Switching". Language in Society 17: 351-373.

Nelson, Theodor H. 1972. "As We Will Think". Reprint in From Memex to Hypertext: Vannevar Bush and the mind's machine, James M. Nyce and Paul Kahn (eds), 245-259. Boston: Academic Press.

Nelson, Theodor H. 1974. Computer Lib/Dream Machines. South Bend/IN: The Distributors.

Nelson, Theodor. 1981. Literary Machines. Sausalito/CA: Mindful Press.

Neurauter-Kessels, Manuela. 2011. “Im/polite Reader Responses on British Online News Sites”. Journal of Politeness Research 7: 187-214. 
O’Driscoll, Jim. 1996. “About Face: A Defence and Elaboration of Universal Dualism”. Journal of Pragmatics 25: 1-32.

O’Driscoll, Jim. 2001. “A Face Model of Language Choice”. Multilingua: Journal of Cross-Cultural and Interlanguage Communication 20 (3): 245-268.

O'Driscoll, Jim. 2011. "Some Issues with the Concept of Face: When, what, how and how much?”. In Politeness across Cultures, Francesca Bargiela-Chiappini and Dániel Z. Kádár (eds), 17-41. Basingstoke: Palgrave Macmillan.

Ord, Jacqueline G. 1989. "Who's Joking? The Information System at Play”. Interacting with Computers 1: 118-128.

O’Sullivan, Patrick B. 2000. "What You Don't Know Won't Hurt Me: Impression Management Functions of Communication Channels in Relationships". Human Communication Research 26: 403-431.

O'Sullivan, Patrick B. and Flanagin, Andrew J. 2003. "An Interactional Reconceptualization 'Flaming' and Other Problematic Communication”. New Media and Society 5 (1): 67-93.

Parks, Malcolm R. and Floyd, Kory. 1996. "Making Friends in Cyberspace". Journal of Communication 46: 80-97. http://jcmc.indiana.edu/vol1/issue4/parks.html, July 5th 2012.

Pinkal, Manfred. 1985. “Kontextabhängigkeit, Vagheit, Mehrdeutigkeit”. In Handbuch der Lexikologie, Christoph Schwarze and Dieter Wunderlich (eds), 27-63. Königstein: Athenäum Verlag.

Pizziconi, Barbara. 2006. "Politeness". In Encyclopedia of Language and Linguistics, Edward K. Brown (ed.), 679-697. Amsterdam: Elsevier.

Quasthoff, Uta M. 1994. “Context”. In Encyclopedia of Language and Linguistics, Ronald E. Asher (ed.), 730-737. Oxford: Pergamon Press.

Reddy, Michael J. 1979. “The Conduit Metaphor: A Case of Frame Conflict in our Language about Language”. In Metaphor and Thought, Andrew Ortony (ed.), 284-297. Cambridge: Cambridge University Press.

Rheingold, Howard. 1993. The Virtual Community: Homesteading on the Electronic Frontier. Reading/MA: Addison-Wesley.

Rice, Ronald E. and Love, Gail. 1987. "Electronic Emotion: Socioemotional Content in a Computer-Mediated Communication Network". Communication Research 14 (1): 85-108.

Rumelhart, David E. and Norman, Donald A. 1981. "Analogical Processes in Learning”. In Cognitive Skills and their Acquisition, John R. Anderson (ed.), 335-359. Hillsdale/NJ: Erlbaum.

Sandig, Barbara. 2000. “Text als prototypisches Konzept”. In Prototypentheorie in der Linguistik: Anwendungsbeispiele - Methodenreflexion - Perspektiven, Martina Mangasser-Wahl (ed.), 93-112. Tübingen: Stauffenburg.

Savas, Perihan. 2011. "A Case Study of Contextual and Individual Factors that Shape Linguistic Variation in Synchronous Text-based Computer-mediated Communication". Journal of Pragmatics 43: 298-313.

Schmid, Hans-Jörg. 2002. "An Outline of the Role of Context in Comprehension". In Anglistentag 2002 Bayreuth. Proceedings, Ewald Mengel, Hans-Jörg Schmid and Michael Steppat (eds), 435-445. Trier: WVT.

Schmidt, Richard. 1980. "Review of Esther Goody, ed., Questions and Politeness: Strategies in Social Interaction”. Regional English Language Centre Journal 11: 100-114.

Schopenhauer, Arthur. 1851. Parerga and Paralipomena. Berlin: s.n. 
Schütte, Wilfried. 2000. "Sprache und Kommunikationsformen in Newsgroups und Mailinglisten". In Sprache und neue Medien, Werner Kallenmeyer (ed.), 142-178. Berlin: Mouton de Gruyter.

Schütte, Wilfried. 2002. "Normen und Leitvorstellungen im Internet: Wie Teilnehmer/-innen in Newsgroups und Mailinglisten den angemessenen Stil aushandeln". In Soziale Welten und kommunikative Stile: Festschrift für Werner Kallmeyer zum 60. Geburtstag, Inken Keim and Wilfried Schütte (eds), 339-362. Tübingen: Narr.

Schulze, Rainer. 1985. Höflichkeit im Englischen: Zur linguistischen Beschreibung und Analyse von Alltagsgesprächen. Tübingen: Narr.

Schwartz, Shalom H. 1992. "Universals in the Content and Structure of Values: Theoretical Advances and Empirical Tests in 20 Countries". In Advances in Experimental Social Psychology 25, Mark P. Zanna (ed.), 1-65. San Diego: Academic Press.

Schwartz, Shalom H., Melech, Gila, Lehmann, Arielle, Burgess, Steven, Harris, Mari and Owens, Vicki. 2001. "Extending the Cross-cultural Validity of the Theory of Basic Human Values with a Different Method of Measurement". Journal of Cross-Cultural Psychology 32 (5): 519-542.

Scollon, Ron and Scollon, Suzanne W. 1995. Intercultural Communication: A Discourse Analysis. Oxford/England: Blackwell.

Selfe, Cynthia L. and Meyer, Paul R. 1991. “Testing Claims for On-line Conferences”. Written Communication 8: 163-192.

Sell, Roger D. ${ }^{2} 2005$, 1992. "Literary Texts and Diachronic Aspects of Politeness". In Politeness in Language: Studies in its History, Theory and Practice, Richard J. Watts, Sachiko Ide and Konrad Ehlich (eds), 109-129. Berlin: Mouton de Gruyter.

Severinson Eklundh, Kerstin. 1986. Dialogue Processes in Computer-Mediated Communication: A Study of Letters in the COM system. University of Linköping: $\mathrm{PhD}$ Thesis.

Shea, Virginia. 1994. Netiquette. San Francisco: Albion Books. http://www.albion.com/bookNetiquette/, July 5th 2012.

Short, John, Williams, Ederyn and Christie, Bruce. 1976. The Social Psychology of Telecommunication. London: John Wiley.

Siegel, Jane, Dubrovsky, Vitaly, Kiesler, Sara and McGuire, Timothy W. 1986. "Group Processes in Computer-Mediated Communication". Organizational Behavior and Human Decision Processes 37: 157-187.

Simmons, Thomas L. 1999. "Face Threats in a Faceless Medium: Negotiating Ideological Parameters in Computer-Mediated Communication". In Language and Ideology: Selected Papers from 6th International Pragamtics Conference, Jef Verschueren (ed.), 514-543. Antwerp: International Pragmatics Association.

Smolowe, Jill. 1995. "Intimate Strangers". Time 145. Special Issue (Welcome to Cyberspace): $20-21$.

Spears, Russell and Lea, Martin. 1994. "Panacea or Panopticon? The Hidden Power in Computer-Mediated Communication”. Communication Research 21: 427-459.

Spencer-Oatey, Helen. 1993. "Conceptions of Social Relations and Pragmatic Research". Journal of Pragmatics 20: 27-47.

Spencer-Oatey, Helen. 2000a. “Introduction: Language, Culture and Rapport Management”. In Culturally Speaking: Managing Rapport through Talk across Cultures, Helen Spencer-Oatey (ed.), 1-8. London/New York: Continuum. 
Spencer-Oatey, Helen. 2000b. "Rapport Management: A Framework for Analysis”. In: Culturally Speaking: Managing Rapport through Talk across Cultures, Helen Spencer-Oatey (ed.), 11-46. London/New York: Continuum.

Spencer-Oatey, Helen. 2002. "Managing Rapport in Talk: Using Rapport Sensitive Incidents to Explore the Motivational Concerns Underlying the Management of Relations". Journal of Pragmatics 34: 529-545.

Spencer-Oatey, Helen. 2005. “(Im)Politeness, Face and Perceptions of Rapport: Unpackaging their Bases and Interrelationships". Journal of Politeness Research 1 (1): 95-118.

Spencer-Oatey, Helen. 2007. “Theories of Identity and the Analysis of Face”. Journal of Pragmatics 39: 639-656.

Sproull, Lee and Kiesler, Sara. 1986. "Reducing Social Context Cues: Electronic Mail in Organizational Communication”. Management Science 32 (11): 1492-1512.

Steele, Guy L. 1983. The Hacker's Dictionary: A Guide to the World of Computer Wizards. Cambridge et al.: Harper and Row.

Stein, Dieter. 2005. "Distanz und Nähe in interaktiver Internetkommunikation". In Chat-Kommunikation in Beruf, Bildung und Medien: Konzepte - Werkzeuge - Anwendungsfelder, Michael Beißwenger and Angelika Storrer (eds), 339-348. Stuttgart: ibidem.

Stephen, Timothy. 1991. "Computer Mediated Communication: Human Relationships in a Computerized World”. Quarterly Journal of Speech 77: 234-236.

Stöckl, Hartmut. 2004. “In between Modes: Language and Image in Printed Media”. In Perspectives on Multimodality, Eija Ventola, Charles Cassily and Martin Kaltenbacher (eds), 9-30. Amsterdam/Philadelphia: John Benjamins.

Stöckl, Hartmut. 2006. "Zeichen, Text und Sinn - Theorie und Praxis der multimodalen Textanalyse". In Textsemiotik: Studien zu multimodalen Medientexten (=Sprache im Kontext), Eva M. Eckkrammer and Gudrun Held (eds), 11-26. Frankfurt/Main: Lang.

Stone, Allucquère R. 1995. The War of Desire and Technology at the Close of the Mechanical Age. Cambridge: MIT Press.

Storrer, Angelika. 2001. "Getippte Gespräche oder dialogische Texte? Zur kommunikationstheoretischen Einordnung der Chat-Kommunikation”. In Sprache im Alltag. Beiträge zu neuen Perspektiven in der Linguistik. Herbert Ernst Wiegand zum 65. Geburtstag gewidmet, Andrea Lehr et al. (eds), 439-465. Berlin: Mouton de Gruyter.

Storrer, Angelika. 2008. "Hypertextlinguistik". In Textlinguistik. 15 Einführungen, Nina Janich (ed.), 211-227. Tübingen: Narr.

Stowasser, Joseph M., Petschenig, Michael and Skutsch, Franz (eds). 2003. Stowasser. Lateinisch-deutsches Schulwörterbuch. München: Oldenbourg Schulbuchverlag.

Strangelove, Michael. 1994. "The Internet, Electric Gaia and the Rise of the Uncensored Self”. Computer-Mediated Communication Magazine 1 (5). http://www.ibiblio.org/cmc/mag/ 1994/sep/self.html, July 5th 2012.

Suler, John R. 2004. “The Online Disinhibition Effect”. http://users.rider.edu/ suler/psycyber/ disinhibit.html\#anonymity, July 5th 2012.

Suler, John R. 2005. "The Basic Psychological Features of Cyberspace. Elements of a Cyberpsychology Model”. http://www-usr.rider.edu/ suler/psycyber/basicfeat.html, July 5th 2012.

Svenning, Lynne L. and Ruchinskas, John E. 1984. “Organizational Teleconferencing”. In The New Media: Communication, Research, and Technology, Ronald E. Rice et al. (eds), 217248. Beverly Hills/CA: Sage. 
Swales, John. 1990. Genre Analysis: English in Academic and Research Settings. Cambridge: Cambridge University Press.

Tannen, Deborah. 1992. That's not what I Meant! How Conversational Style Makes or Breaks your Relations with Others. London: Virago Press.

Tanskanen, Sanna-Kaisa. 2007. "Metapragamtic Utterances in Computer-Mediated Interaction”. In Metapragmatics in Use, Wolfram Bublitz and Axel Hübler (eds), 87-106. Amsterdam/Philadelphia: John Benjamins.

Taylor, Jacqueline. 2000. "Electronic Mail, Communication and Social Identity: A Social Psychological Analysis of Computer-Mediated Group Interaction". In Words on the Web: Computer Mediated Communication, Lyn Pemberton and Simon Shurville (eds), 96-105. Exeter/Portland: Intellect Books.

Terkourafi, Marina. 2008. "Towards a Unified Theory of Politeness, Impoliteness, and Rudeness". In Impoliteness in Language: Studies on its Interplay with Power in Theory and Practice, Derek Bousfield and Miriam A. Locher (eds), 45-74. Berlin: Mouton de Gruyter.

Thomas, Jenny. 1983. "Cross-cultural Pragmatic Failure”. Applied Linguistics 4 (2): 91-112.

Thomas, Jenny. 1995. “Theories of Politeness”. In Meaning in Interaction: An Introduction to Pragmatics, Jenny Thomas (ed.), 149-181. Harlow: Longman.

Thurlow, Crispin, Lengel, Laura and Tomic, Alice. 2004. Computer-Mediated Communication: Social Interaction and the Internet. Los Angeles et al.: Sage.

Ting-Toomey, Stella. 1988. "Intercultural Conflict Styles: A Face Negotiation Theory". In Theories in Intercultural Communication, Young Y. Kim and William B. Gudykunst (eds), 213235. Newbury Park/CA: Sage.

Ting-Toomey, Stella. 1994. "Face and Facework: An Introduction". In The Challenge of Face Work: Cross-cultural and Interpersonal Issues, Stella Ting-Toomey (ed.), 1-5. Albany: State University of New York Press.

Tomasello, Michael. 2008. Origins of Human Communication. Cambridge/MA: MIT Press.

Tracy, Karen. 1990. “The Many Faces of Facework”. In Handbook of Language and Social Psychology, Howard Giles and W. Peter Robinson (eds), 209-226. Chichester: John Wiley.

Tulving, Endel. 1972. "Episodic and Semantic Memory". In Organization of Memory, Endel Tulving and Wayne Donaldson (eds), 381-402. New York: Academic Press.

Tulving, Endel. 1983. Elements of Episodic Memory. Oxford: Clarendon Press.

Turkle, Sherry. 1995. Life on the Screen: Identity in the Age of the Internet. New York: Touchstone.

Turnage, Anna K. 2007. "Email Flaming Behaviors and Organizational Conflict". Journal of Computer-Mediated Communication 13 (1). http://jcmc.indiana.edu/vol13/issue1/turnage. html, July 5 th 2012.

Vater, Heinz. 1992. Einführung in die Textlinguistik: Struktur, Thema und Referenz in Texten. München: Fink.

Vilkki, Liisa. 2006. "Politeness, Face and Facework: Current Issues". SKY Journal of Linguistics 19 (special supplement): A Man of Measure: Festschrift in Honour of Fred Karlsson on his 60th Birthday): 322-332. http://www.ling.helsinki.fi/sky/julkaisut/SKY2006_1/1.4.7.\% 20VILKKI.pdf, July 5th 2012.

Walther, Joseph B. 1992. "Interpersonal Effects in Computer-Mediated Interaction: A Relational Perspective”. Communication Research 19 (1): 52-90. 
Walther, Joseph B. 1996. "Computer-Mediated Communication: Impersonal, Interpersonal, and Hyperpersonal Interaction”. Communication Research 23 (1): 3-43.

Walther, Joseph B., Anderson, Jeffrey F. and Park, David W. 1994. "Interpersonal Effects in Computer-Mediated Interaction: A Meta-Analysis of Social and Antisocial Communication". Communication Research 21: 460-487.

Walther, Joseph B. and Burgoon, Judee K. 1992. "Relational Communication in Computer-Mediated Interaction”. Human Communication Research 19: 50-88.

Walther, Joseph B. and Parks, Malcolm R. 2002. "Cues Filtered in, Cues Filtered out - Computer-Mediated Communication and Relationships". In Handbook of Interpersonal Communication, Mark L. Knapp and John A. Daly (eds), 529-563. Thousand Oaks/CA: Sage.

Watts, Richard J. 2003. Politeness. Cambridge: Cambridge University Press.

Watts, Richard J. 2005. “Linguistic Politeness Research: Quo vadis?”. In Politeness in Language: Studies in its History, Theory and Practice, Richard J. Watts, Sachiko Ide and Konrad Ehlich (eds), xi-xlvii. Berlin: Mouton de Gruyter.

Watts, Richard J., Ide, Sachiko and Ehlich, Konrad (eds). ${ }^{2} 2005$, 1992. Politeness in Language: Studies in its History, Theory and Practice. Berlin: Mouton de Gruyter.

Watzlawick, Paul, Bavelas, Janet B. and Jackson, Don D. 1967. Pragmatics of Human Communication: A Study of Interactional Patterns, Pathologies, and Paradoxes. New York: Norton.

Weedman, Judith. 1991. "Task and Non-task Functions of a Computer Conference Used in Professional Education: A Measure of Flexibility”. International Journal of Man-Machine Studies 34: 303-318.

Werkhofer, Konrad T. ${ }^{2} 2005$, 1992. “Traditional and Modern Views: The Social Constitution and Power of Politeness". In Politeness in Language: Studies in its History, Theory and Practice, Richard J. Watts, Sachiko Ide and Konrad Ehlich (eds), 155-199. Berlin: Mouton de Gruyter.

Werry, Chris. 1996. "Linguistic and Interactional Features of Internet Relay Chat". In ComputerMediated Communication: Linguistic, Social and Crosscultural Perspectives, Susan Herring (ed.), 47-63. Amsterdam/Philadelphia: John Benjamins.

Werry, Chris and Mowbray, Miranda (eds). 2001. Online Communities: Commerce, Community Action, and the Virtual University. Upper Saddle River/N.J.: Prentice Hall.

Widdowson, Henry G. 2007. Discourse Analysis. Oxford: Oxford University Press.

Wilbur, Shawn P. 1997. "An Archaeology of Cyberspaces: Virtuality, Community, Identity”. In Internet Culture, David Porter (ed.), 5-22. New York: Routledge.

Wilkins, Harriet. 1991. “Computer Talk: Long-Distance Conversations by Computer”. Written Communication 8: 56-78.

Wolfson, Nessa. 1988. “The Bulge: A Theory of Speech Behaviour and Social Distance”. In Second Language Discourse: A Textbook of Current Research, Jonathan Fine (ed.), 21-38. Norwood/N.J.: Ablex.

Yule, George. 1996. "Politeness and Interaction". In Pragmatics, George Yule (ed.), 59-69. Oxford: Oxford University Press.

Yum, Young-ok and Hara, Kazuya. 2005. "Computer-Mediated Relationship Development: A Cross-cultural Comparison”. Journal of Computer-Mediated Communication 11 (1). http:// jcmc.indiana.edu/vol11/issue1/yum.html, July 5th 2012. 


\section{Further electronic sources}

URL 1: http://www.spiegel.de/netzwelt/web/0,1518,709694,00.html, July 5th 2012.

URL 2: http://eetiquette.de/, July 5th 2012.

URL 3: http://groups.google.com/support/bin/answer.py?hl=enandanswer=46461, July 5 th 2012.

URL 4: http://www.network54.com/Forum/358339/, July 5th 2012.

URL 5: http://www.thestudentroom.co.uk/forums, July 5th 2012.

URL 6: http://www.thestudentroom.co.uk/forumdisplay.php?f=89, July 5th 2012.

URL 7: http://www.thestudentroom.co.uk/showthread.php?p=20270280\#post20270280, July 5 th 2012.

URL 8: http://www.thestudentroom.co.uk/faq.php?faq=rep, July 5th 2012.

URL 9: http://www.thestudentroom.co.uk/faq.php?faq=rules, July 5th 2012. 


\section{Name index}

A

Androutsopoulos, Jannis K. 13,35

Arndt, Horst 100, 108, 110, 122 Arundale, Robert B. 50, 77, 79, 113

Austin, Paddy $\quad 79,89,91,122$

Avgerinakou, Anthi 21-22, 24, $35,42-43,100,124$

\section{B}

Baker, Paul 101, 129

Bargh, John A. 20, 34

Bargiela-Chiappini, Francesca $60,67,70,74$

Baron, Naomi 19, 28

Baym, Nancy $19,21,35,40-41$, 47

Beißwenger, Michael 11, 13, 28, 128

Benwell, Bethan 27, 39, 42, 129

Blum-Kulka, Shoshana 55, 65

Bolter, Jay 6, 8-9

Brown, Penelope $54,58-66$, 71-77, 84, 86, 89-94, 115, 123, 129, 164, 260

Brown, Roger 123-124

Bublitz, Wolfram 1, 8-9, 79, 106-107

Bush, Vannevar 5-7

C

Chandler, Daniel 18-19, 114

Claridge, Claudia 130-131

Clark, Herbert H. 106, 110

Collins, Mauri 20-21

Coupland, Nikolas $\quad 68,115$

Crystal, David $\quad$ 13, 26, 28, 114

Culnan, Mary 11, 20, 22, 25

Culpeper, Jonathan 89-90,

92-95, 97-99
D

Derks, Daantje 20, 26, 28, 34

Donath, Judith S. 111, 121

Douglas, Karen M. 7, 21, 35

Duranti, Alessandro 105-106

E

Eelen, Gino 54, 79-80, 90, 94

Erickson, Thomas 37,129

F

Floyd, Kory 39, 42

Foucault, Michel 47, 114

Fraser, Bruce $\quad 54-58,64-65,75$

G

Goffman, Erving 44, 46-47, 54, $66-73,75-76,84,113,115,117$, 164,260

Goodwin, Charles $\quad$ 105-106

Graham, Sage L. $\quad 28,50,130$

Grice, Herbert P. 47, 57-59, 75,100

$\mathrm{H}$

Held, Gudrun 55-56

Herring, Susan 3, 93,10-13, 22, 27-28, 34, 39-40, 45-47, 107,

$111,121,124,127-139,131$

Hiltz, Starr 22, 26

Hoffmann, Christian $\quad 8-9$

Holly, Werner 9, 66, 128

Holmes, Janet $\quad 65-66,98$

J

Janney, Richard 100, 108, 110, 122

Jaworski, Adam 68, 115

Jones, Rodney H. 105, 110, 125

Jones, Steven G. $\quad 39,130$
K

Kasper, Gabriele $\quad 74,81,86,93$, 96, 106

Kendall, Lori 40, 114

Kienpointner, Manfred 89, 93, 95-99

Kiesler, Sara $\quad$ 19-20, 22, 24, 48

Kollock, Peter 2, 31, 46-48

Kuhlen, Rainer 9,13

L

Lachenicht, Lance $\quad 89-91,97$

Lakoff, Robin T. 54-55, 57-60, 75, 94

Lea, Martin $\quad 21-22,24,26,28$, $35,42,111$

Leech, Geoffrey N. 55, 57-60, 75, 93, 96-98

Locher, Miriam A. $54,74,76$, 79-81, 83-87, 89, 93-94, 97, 101-102, 114, 128, 261

M

Maricic, Ibolya $\quad 129,131$

Markus, M. Lynne 11, 20, 22, 25

McCormick, John W. 24, 29

McCormick, Naomi B. 24, 29

McGarty, Craig 7, 21, 35

McKenna, Katelyn Y. A. 20, 34

McLuhan, Marshall 9, 18

Meier, Ardith J. $\quad 58,88$

Meyrowitz, Joshua 18-19, 42-43, 115

Mills, Sara $\quad 54,89,99$

Murray, Denise E. 9, 12, 28, 107

$\mathrm{N}$

Nelson, Theodor $\quad 6,8-9$ 
$\mathrm{O}$

O'Driscoll, Jim 6o, 71, 75-76, 79, 84, 117

O'Sullivan, Patrick 34,100

\section{P}

Parks, Malcolm 25, 39, 42

R

Rheingold, Howard 2, 7, 25, 29, 38-39, 47

\section{S}

Savas, Perihan 106, 129

Schmid, Hans-Jörg $\quad$ 65, 106

Schütte, Wilfried $13,28,37$, 42-43

Sell, Roger 53,80

Shea, Virginia 47,101
Short, John 21, 26

Siegel, Jane 21, 24

Smith, Marc A. 2, 31, 46-48

Spears, Russell 21-22, 26, 28, $35,42,111$

Spencer-Oatey, Helen 79, 87, $108,113,123$

Stephen, Timothy 10,58

Stokoe, Elisabeth $\quad 27,39,42,129$

Storrer, Angelika $\quad 6,8-10$

Suler, John R. 17, 34, 37, 45, 126

\section{$\mathrm{T}$}

Tannen, Deborah 1-2, 60, 109

Tanskanen, Sanna-Kaisa 17, 130

Taylor, Jacqueline $\quad 23-24,129$

Terkourafi, Marina 95, 97

Thomas, Jenny $19,55,63,95$
Thurlow, Crispin $\quad 7,13,18$, 21-23, 27-28, 34, 37-39, 100, 113

Ting-Toomey, Stella 74, 113

Tracy, Karen 66, 113

Tulving, Endel 109-110

W

Walther, Joseph B. $\quad 20,22-27$

Watts, Richard J. 53-54, 57-59, 69, 71-74, 79-87, 89, 93-94, 97, 101-102, 108, 123, 261

Werkhofer, Konrad 55, 65, 72,88

Werry, Chris 35, 39

Y

Yule, George $\quad 1,61,63$ 


\section{Subject index}

A

acronym $26-27,152$

aggravating language 90

aggravation

negative 90-91

positive 90-91

animator

see production format

anonymity 21,34

ARPANET 7

AUGMENT 7

author see production format

avatar 29, 111, 114-115

\section{B}

bald-on-record impoliteness 92

bald-on-record strategy 91

banter (principle) 93, 96-100, 102

basic human need

$\sim$ for association 60,75 ,

$87,115,158,164,202,247$,

251, 261

$\sim$ for dissociation $\quad 60,75$,

115-116, 164, 202, 247, 249,

251, 261

behavior

appropriate 53, 74, 85-87,

97, 102-103, 110, 171-173,

252, 256-257, 261

inappropriate $89,92-96$,

99-103, 173, 252, 256-257, 262

interpersonal 70,87, 159, 171,262

marked 85, 102, 255

negatively marked $\sim 85,89$,

94, 103, 253, 255, 261, 263

non-polite $~ 85-86$ positively marked $\sim \quad 85,102$, 250, 255

unmarked 85, 171, 173,

254-255, 262

C

CMC see computer-mediated communication

common ground $73,87,110$, $123,126,149,181,248$

communication

computer-mediated (CMC) $4-6,10-13,16-17,19-28$, $32-37,39,44-45,49,89$, 100-101, 106, 108-109, 114, 122, 128-129, 139, 141, 152,

259, 262-263

face-to-face $(\mathrm{Ftf}) \sim 20$,

23-24, 45, 109

online 10, 13, 259-26o, 263

communicative purpose $\quad$ 137$138,153,262$

community

discourse $~ 40$

online 39, 43, 50

speech 127

virtual 39, 46

$\sim$ of practice $40-42,46,50$, 87,260

computer-mediated discourse

(CMD) 11-12

computer-mediated discourse analysis (CMDA) $\quad 5,10-12$

context

dimensions of $\sim 107$

emergent $\sim 107$

interpersonal 107, 122-126, 261

personal 107, 123, 125-126, 261 conversational contract view 56,75

conversational maxim view

55-57

cooperation 10, 47, 59, 69,

84,90

(Grice's) cooperative principle

(CP) $57-58,75$

corpus compilation 130

cues-filtered in approach

see cues-filtered out

approaches

cues-filtered out approaches 6 ,

$17,19-20,23,35$

cultural background 108-109,

111, 126

culture $18-19,64-65,72,81,89$, 95, 97, 99, 108-109

\section{D}

deficit approaches see cues-filtered out approaches

de-individuation theory 21

demographic variable 20

DIRECTIVE $139,150,152,154$, 159

discourse $14,21,47,49,81,89$, $127,131,164$

E

email 30, 34, 37, 102, 240, 259

emoticon 24, 26, 28, 47, 111, 150

(first) entry 14, 31, 33, 44, 47,

131, 148-154, 157, 159-161, 168,

255-256

environment (extra-medial /

static ) 107

ethnography of speaking 107

EXPRESSIVE $\quad 150,154$ 
F

face

$\sim$ attack act 89,91

$\sim$ claim 84, 117-121, 140-143, 145-146, 153, 159, 163-164, 168, 260-261

$\sim$ constitution 112-115, 119, 209

$\sim$ loss 62,70

$\sim$ maintaining / maintenance $74,96,98$

$\sim$ saving 73

$\sim$ saving view 55,58

$\sim$ want $59-61,63,77,92,169$ negative 60-61, 63, 72-73, $76,91-92$

positive 60-61, 72-73, 92, 158,215

Facebook 29, 111

face threatening act (weightiness of ) $61-65,74,87,90-93$, 96

facework

negative 147, 158, 161, 164, 202, 234, 238

positive 170, 176-177,

179, 181, 202, 212, 215, 238, 246, 249

flaming (pseudo- / mock- $\sim$ )

21, 24, 49, 51, 89, 99-103, 254

flame war $101,147,149,235$

forum 11-12, 14, 42, 48, 131, 153, 208, 210-211

FTA see face threatening act

\section{G}

greeting 44, 139, 148, 159, 170 Grice's maxims $\quad 47,57,59,100$ group 12-13, 21, 30, 32, 37-41, $47,50,69,72,108-109,121-$ $123,128-129,248,256-257$, 260

H

heading $13-16,44,49,132$, $137-140,159,250$

hybridization 42, 45

hyperlink 9, 125

hypermedia 9

hypertext 5-10, 37
I

identity

online 114

$\sim$ construction $77,112,114$, 129

impoliteness $53,79-80,83$,

89-90, 92-103, 252, 254,

260-261, 263

instant messaging 129

interaction quotient (IQ) 165166, 251, 257

interactivity 10,164

Internet $\quad 5-7,22,37,39,128-130$, 201

interpersonal relations 20,22 , $32,53,58,75,79-80,83-85$, 88, 105-106, 122-123, 128-130, $137,159,163,167,247,251$, 259-261

irony principle (IP) 93

K

knowledge conceptual 110 declarative $~ 109$ episodic 109, 128 factual 109-110 linguistic 109 procedural $\sim 109$ semantic $\sim 110$

L

label 30, 45, 111

linearity (compositional / perceptive $\sim) 8$

lurker see production format

M

media richness theory 21

medium 9-10, 12, 18-19, 22, 28, 35, 43

message board 12-17

$\sim$ corpus $3,130-135,253$

$\sim$ member 3,261

$\sim$ moderator $12,31,46$, 179, 250

$\sim$ post (prototypical) 33

$\sim$ template $29,111,121,125$, 141

metapragmatic utterance 127 $128,149,159,217$ mock-impoliteness 93, 96-102, $176,252,261$

mock-politeness $\quad$ 92-93, 252, 261

$\mathrm{N}$

negative face see face neg rep see reputation netiquette $28,46-49,153$ netlingo 25, 27-28, 114, 139, 152, 207

netspeak see netlingo newbie $45-46,50,107,110$, 122,250

newsgroup see message board nickname 21, 29, 111, 114-115,

135,142

\section{O}

observer's paradox 128 off-record strategy $\quad 65,91$

online persona 112,118

outside observer $42,125,128$, 172

over-politeness $83,89,94$

$\mathbf{P}$

participant observation

(participant observer) 128, $166,172-173,251,254,262$

personal attack $48-49,51,153$

politeness

first-order $80-81,83$

linguistic $\sim 74$

negative 62-63, 65, 86, 91-92, 115

positive 62-63, 65, 72-73,

$86,91-92,115$

rules of $\sim 53,57-58$

second-order 81

withhold 92-93

$\sim$ principle $57-58,93$

$\sim$ strategy $59,73,92-93$

$\sim$ theory $58,64,74$

positive face see face pos rep see reputation post

erratic $~ 166$

evaluated $\sim 166$

evaluating 166 


\begin{tabular}{|c|c|c|}
\hline postmodernism $\quad 55,79,87-88$, & reputation & $\mathrm{T}$ \\
\hline 102,260 & positive 142-144, 147 & technological determinism \\
\hline pragmatics & negative $\sim 142-144,147$ & $17-19$ \\
\hline interpersonal $\sim 259$ & ritual insults see banter & The Student Room (TSR) \\
\hline meta- 127-128 & rudeness $53,89-91,93-97$, & $14-17,29-33,38,40-42$, \\
\hline pragmatic competence 57,95 & $101-102,261,263$ & $45-50,109-110,130-135,165$ \\
\hline prepragmatic approach 55,88 & rules of conversation $\quad 57-58$ & 249 \\
\hline principal see production & & thread \\
\hline format & S & $\sim$ starter $16,30,44,139-141$, \\
\hline production format $44-45$ & sarcasm $59,92-93$ & $143^{-145,152,155}$ \\
\hline profile & self-disclosure $21,34-35$ & $\sim$ title $132-134,137-138$ \\
\hline empty $\sim 141$ & self-portrayal $110-112,122$ & time stamp 16,32 \\
\hline informative $\sim 141$ & signature $31-32,111,157-158$, & troll(ing) $\quad 49,51,121-122,141$ \\
\hline & 260 & TSR see The Student Room \\
\hline $\mathbf{R}$ & smiley $\quad 24,26,150-152,159,260$ & \\
\hline recipient & social constructionism $\quad 18-19$ & $\mathrm{U}$ \\
\hline ratified $\sim 44$ & social network sites 35 & universality $\quad 59,64,81$ \\
\hline unratified $\sim 44$ & social norm view $\quad 55-56,75,88$ & Usenet 13,102 \\
\hline relational work (types of $\sim$ ) & social presence theory $\quad 20-21$ & \\
\hline $83-86,88-89,97,102,108$ & social roles $42,45,50$ & $\mathbf{W}$ \\
\hline $\begin{array}{l}136,163-164,247-248,252- \\
256,261-263\end{array}$ & $\begin{array}{l}\text { speech act } 65,75,86 \\
\text { surplus approach to politeness }\end{array}$ & $\begin{array}{l}\text { warnings level } 144,261 \\
\text { weblish see netlingo }\end{array}$ \\
\hline REPRESENTATIVE $\quad 150,154$ & $\begin{array}{l}\text { see relational work } 85-86 \text {, } \\
254\end{array}$ & weblog $11,13,111$ \\
\hline
\end{tabular}


This descriptive and comprehensive study on the discursive struggle over interpersonal relations in online message boards is located at the fascinating interface of pragmatics and computer-mediated discourse - a research area which has so far not attracted much scientific interest. It sets out to shed light on the question how interpersonal relations are established, managed and negotiated in online message boards by giving a valid overview of the entire panoply of interpersonal relations (and their interrelations), including both positively and negatively marked behavior. With the first part of the book providing an in-depth discussion and refinement of the pivotal theoretical positions of both fields of research, students as well as professionals are (re-)acquainted with the subject at hand. Thus supplying a framework for the ensuing case study, the empirical part displays the results of the analysis of 50 threads (ca. 300,000 words) of a popular British message board.

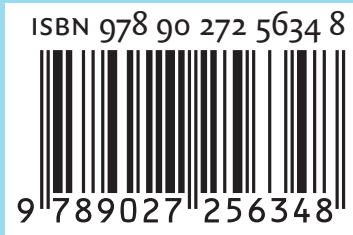

\section{John Benjamins Publishing Company}

